\title{
The impact of reward value on early sensory processing and its interaction with selective attention
}

\author{
Dissertation \\ for the award of the degree \\ "Doctor rerum naturalium" \\ of the Georg-August-Universität Göttingen \\ within the doctoral program Systems Neuroscience \\ of the Georg-August University School of Science (GAUSS)
}

submitted by
Roman Vakhrushev

From Yaroslavl, Russia

Göttingen 2021 


\section{Thesis Committee}

Dr. Arezoo Pooresmaeili

European Neuroscience Institute, Grisebachstrasse 5, 37077 Goettingen, Germany

Prof. Dr. Annekathrin Schacht

Georg-Elias-Müller-Institute of Psychology, Georg-August University, Wilhelmsplatz 1, 37073 Goettingen, Germany

Prof. Melanie Wilke

Cognitive Neurology University Medical Center Göttingen, Robert-Koch-straße 40, 37075

Göttingen, Germany

\section{Members of the Examination Board}

$1^{\text {st }}$ Referee: Dr. Arezoo Pooresmaeili

European Neuroscience Institute, Grisebachstrasse 5, 37077 Goettingen, Germany

$2^{\text {nd }}$ Referee: Prof. Dr. Annekathrin Schacht

Georg-Elias-Müller-Institute of Psychology, Georg-August University, Wilhelmsplatz 1, 37073 Göttingen, Germany 


\section{Further members of the Examination Board}

Prof. Dr. Andrea Antal

Clinical Neurophysiology University Medical Center Göttingen, Robert-Koch-Straße 40, 37075 Göttingen, Germany

Prof. Dr. Alexander Gail

German Primate Center, Kellnerweg 4, 37077 Göttingen, Germany

Dr. Igor Kagan

German Primate Center, Kellnerweg 4, 37077 Göttingen, Germany

Dr. Caspar Schwiedrzik

European Neuroscience Institute, Grisebachstrasse 5, 37077 Goettingen, Germany

Date of oral examination: November $12^{\text {th }}, 2021$ 


\section{Table of Contents}

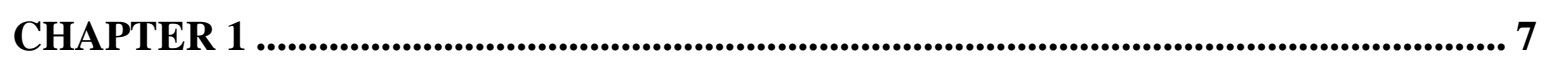

1.1 GENERAL INTRODUCTION ……………………………..................................... 7

1.2 OBJECTIVES AND STRUCTURE OF THE CURRENT THESIS ............................................ 21

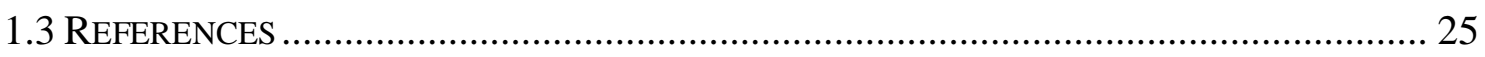

CHAPTER 2 DIFFERENTIAL EFFECTS OF INTRA-MODAL AND CROSS-

MODAL REWARD VALUE ON VISUAL PERCEPTION: ERP EVIDENCE.......... 32

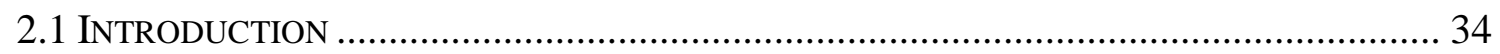

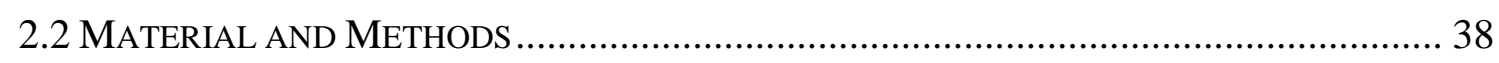

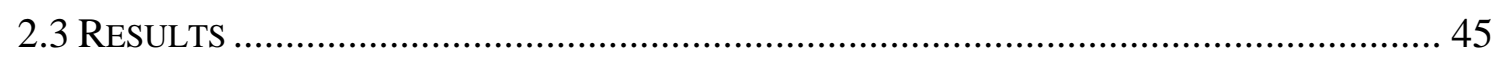

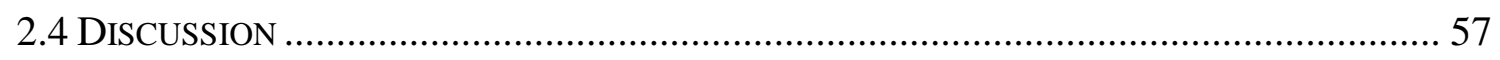

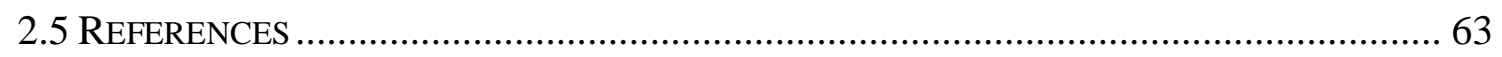

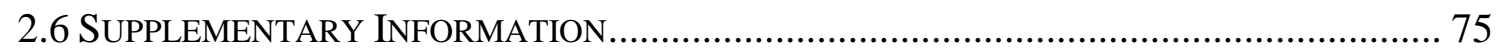

CHAPTER 3: SPATIOTEMPORAL CHARACTERISTICS OF THE CAPTURE OF ATTENTION BY REWARD CUES FROM DIFFERENT SENSORY MODALITIES

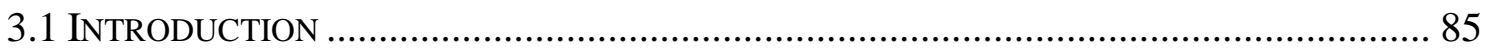

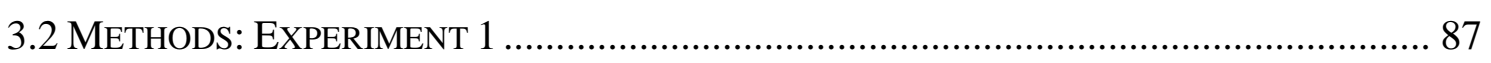

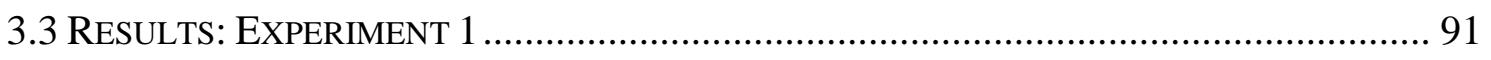

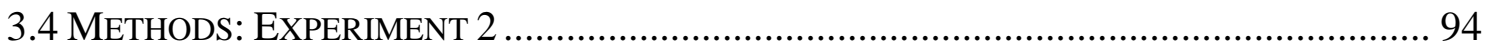

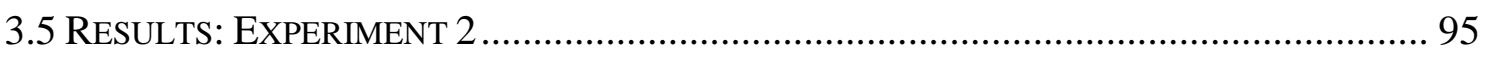

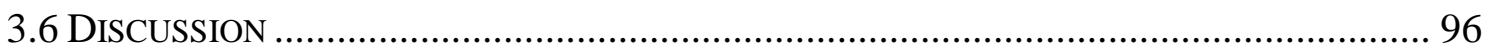

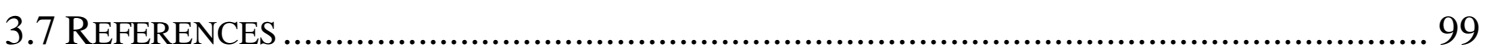

CHAPTER 4: INTERACTION OF SPATIAL ATTENTION AND ASSOCIATIVE REWARD VALUE OF AUDIOVISUAL STIMULI .................................................... 111

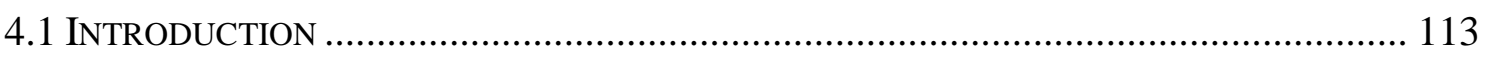

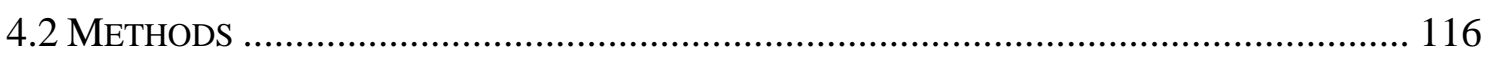

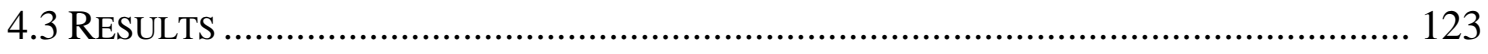

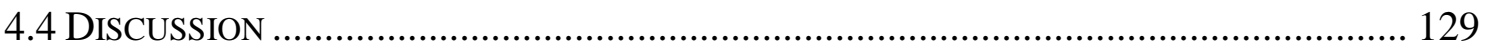

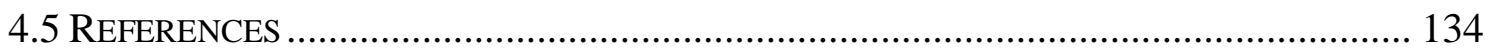

CHAPTER 5: GENERAL DISCUSSION.................................................................... 137 
5.1 SUMMARY OF THE RESULTS

5.2 INTRA- AND CROSS-MODAL REWARD EFFECTS ON BEHAVIORAL AND

ELECTROPHYSIOLOGICAL INDICES

5.3 INTERACTION OF REWARD EFFECTS WITH SELECTIVE ATTENTION.

5.4 EMBEDDING INTRA- AND CROSS-MODAL REWARD EFFECTS IN COGNITIVE MODELS OF PERCEPTION

5.5 FINAL CONCLUSIONS AND FUTURE PERSPECTIVES.

REFERENCES. 


\begin{abstract}
Reward value affects the earliest stages of sensory perception. Whereas a host of previous studies have investigated the underlying mechanisms of reward-driven modulation of visual perception, reward effects in other sensory modalities have remained underexplored. Specifically, it has remained unknown how reward signals should be coordinated and communicated across sensory modalities. The current $\mathrm{PhD}$ thesis aimed to gain insight into the underlying mechanisms of reward-driven modulation of perception and its interaction with attention across sensory modalities. To this end, three experiments were conducted to identify the behavioral and electrophysiological correlates of reward effects. In Study 1, we found that high reward, task-irrelevant visual cues (intra-modal) elicited an early suppression of visual event-related potentials (ERPs). High reward auditory cues (crossmodal), on the other hand, led to a later modulation of visual ERPs and facilitated behavioral performance. Study 2 tested the dependence of reward effects on the spatial and temporal arrangement of intra-modal and cross-modal cues relative to the target, and showed that each reward cue maximally exerts its effect under a specific size of attentional focus. Study 3 explicitly manipulated the spatial attention and tested how reward associations of an audiovisual stimulus influence the allocation of attention. We found that auditory rewards enhanced the attentional modulation of both visual and auditory ERPs. Interestingly, although visual rewards did not lead to a distinguishable ERP modulation, they led to strong modulations when they were combined with auditory rewards, suggesting that integration across modalities boosts the reward effects. Taken together, the current $\mathrm{PhD}$ thesis identified the behavioral and neural signatures of reward-driven modulation of perception under different modes of reward signaling and different degrees of attentional engagement. Our findings inspire a two-stage model of reward processing, with local, intra-modal reward effects occurring at an early stage and long-range, multimodal reward effects arising at a later stage. Cross-modal reward signals have important ramifications for clinical applications where the impaired function of one sense can be rehabilitated by motivational signals conveyed through another sensory modality.
\end{abstract}




\section{Chapter 1}

\subsection{General introduction}

\subsubsection{A brief introduction to the key question of the thesis}

A fundamental determinant of the well-being of every living organism is the ability to select and integrate the most relevant and important information while filtering out irrelevant signals from the environment (Desimone and Duncan, 1995; Reynolds et al., 1999). In particular, this selection mechanism seeks to extract from the environment variables that indicate the value or, in other words, aims at maximizing reward outcomes (Berridge and Kringelbach, 2015; Dawkins, 2016). The pursuit of reward is one of the main mechanisms that drive behavior to interact with the environment (Pessoa and Engelmann, 2010; Takikawa et al., 2002). To maximize the reward outcome, an organism has to rely on external signals (also called “cues") that are processed by specialized sensory receptors. However, these receptors cannot infer the value of the signal directly. In perspective, while walking in the forest, a rustling sound may indicate a danger like a wild animal, but it could also be due to the flow of wind that moves leaves or a friend that tries to approach in disguise in order to surprise us. The activation of a single sensory receptor is thus not sufficient to determine the specific value or importance of the sound. A more reliable way for this evaluation is to gather more information. That could be done either by detecting other sounds or by incorporating information from other sensory modalities. In our case, one can turn the head and look at the source of the sound (visual modality) or notice pressure from the wind on the skin (tactile modality). The principle of integrating sensory information from multiple sensory modalities is reported at the level of behavior (Cornelio et al., 2021; Frens et al., 1995; Goldring et al., 1996) down to a single cell (Meredith and Stein, 1986; Stein and Meredith, 1993; Woods and Recanzone, 2004). While it is known that integration of sensory information across modalities enhances the precision of perceptual decisions, it has remained unknown how information related to the value/importance of stimuli is encoded and coordinated across modalities. For instance, other environmental sounds may provide congruent or incongruent information regarding whether a rustling sound in the forest signals impending danger or not. In this case, information regarding the value or importance of one cue (sound of the wind) can be influenced by cues in the same sensory modality, i.e. intramodally. Similarly, cues from a different modality such as vision, for instance, the sight of our friends' bike somewhere nearby or the remains of an animal, could affect how we 
estimate the value/importance of a rustling sound, an influence that occurs cross-modally (Anderson, 2016a; Cheng et al., 2020; Pooresmaeili et al., 2014; Sanz et al., 2018). How these two types of reward-related information can influence our perceptual and value-based decisions has remained largely unknown. This gap in our knowledge is conceptually important since many existing neuroeconomic models of decision-making suggest that the expected value of an object or a state is computed irrespective of its specific sensory properties. For instance, according to the prospect theory (Fox and Poldrack, 2009; Kahneman and Tversky, 2013), the expected utility of a certain object or state is equal to the product of the magnitude and probability of its associated reward independent of the specific sensory properties of the cues that signal that reward. This is in contrast to the findings of psychophysical and neuroimaging/neurophysiological studies demonstrating the dependence of reward effects on sensory properties of stimuli and a tight interaction between the valuation system and primary sensory areas of the brain (Anderson et al., 2011a; Braver et al., 2014; Failing and Theeuwes, 2018; Pessoa, 2015; Serences, 2008; Shuler and Bear, 2006). Furthermore, previous literature has shown that reward value and selective attention closely interact with each other (Maunsell, 2004). Since attentional effects have dependencies on sensory properties of stimuli including their location, perceptual organization (Desimone and Duncan, 1995; Reynolds et al., 1999) and sensory modality (Alais et al., 2006), effects of reward value are also expected to depend on sensory properties of stimuli. Against this background, the central aim of this thesis is to understand how the modulation of perception by reward depends on the specific properties of reward cues, namely their sensory modality, and how this interaction is influenced by selective attention. This is an important question since in natural environments reward value can be signaled through multiple sensory modalities but it is currently unknown how reward-driven effects interact and combine across senses.

\subsubsection{Components of reward signals}

Reward signals, like any other signal, have sensory and attentional components, as well as a unique value component (Schultz, 2015). These three components contribute to the processing of reward signals: with the sensory component, the reward signal evokes responses in early sensory areas (visual, auditory, olfactory, gustatory, somatosensory), and with the attentional component, the reward signal gains access to the limited informationprocessing resources (Nakayama, 1990). Finally, the value component, like an attentional component, helps the signal to gain access to limited processing resources. However, this gain relies on the magnitude and probability of reward outcomes (as in prospect theory) 
rather than on its sensory properties. Importantly, sensory and attentional components can be derived from perceptual characteristics of the signal (e.g., red color). The value component, on the other hand, requires additional internal processing since there are simply no specialized receptors to detect this value component directly (Schultz, 2015). Instead, perceptual systems evaluate value components later in sensory processing (Wise, 2004), where the previous knowledge gained through experience with positive or negative reward outcomes is integrated with the information related to the sensory properties of cues, and as shown recently, the latter occurs across different sensory modalities (Cheng et al., 2020; Stine et al., 2020).

Evolutionary, there are three reasons why perception does not have a built-in reward evaluation system on its own. First, reward value is a subjective property that varies across organisms (Sobal, 1998) and depends on their internal (homeostasis) and external (goals) states that change over time (Peters and Büchel, 2010; Schultz, 2013). For example, the initial behavioral drive from a given homeostatic imbalance (e.g., hunger) fades rapidly when fulfilled. Thus, after balance is restored, sensory inputs that were previously valuable will lead to a different reward outcome, even to a punishment. Second, reward value is discounted over time (Ainslie, 1975; Shadmehr et al., 2010; Wittmann et al., 2007). An immediate reward has a higher value than a delayed reward (Frederick et al., 2002). Here immediate reward affects the homeostatic imbalance directly, while with the delay, chances are higher that this imbalance will be fulfilled or an alternative solution will be found. Third, the value of an item or a state, even if it is immediate, involves uncertainty or risk (Rangel et al., 2008). Signals previously followed by a reward do not predict reward with certainty, and instead, they indicate the probability of a reward outcome. In order to accommodate the probabilistic nature of reward signals, perceptual systems seek to optimize sensory processing in such a way that signals aligned in time and/or space with the reward contribute the most to perceptual (Gold and Shadlen, 2007) and value-based decision making (Rangel et al., 2008). Additionally, alignment of reward signals in time allows the perceptual system to integrate different properties of reward outcomes into a coherent representation (Basten et al., 2010; Cheng et al., 2020; Sanz et al., 2018; Stine et al., 2020). Due to the multifaceted nature of reward signals, it is important to study reward effects on behavioral and neuronal responses at multiple levels encompassing learning of reward associations, representation of reward value during value-based decisions, and their integration with sensory properties of stimuli. In the following sections, I will briefly describe these levels and will then turn to their underlying neural mechanisms and cognitive models. 

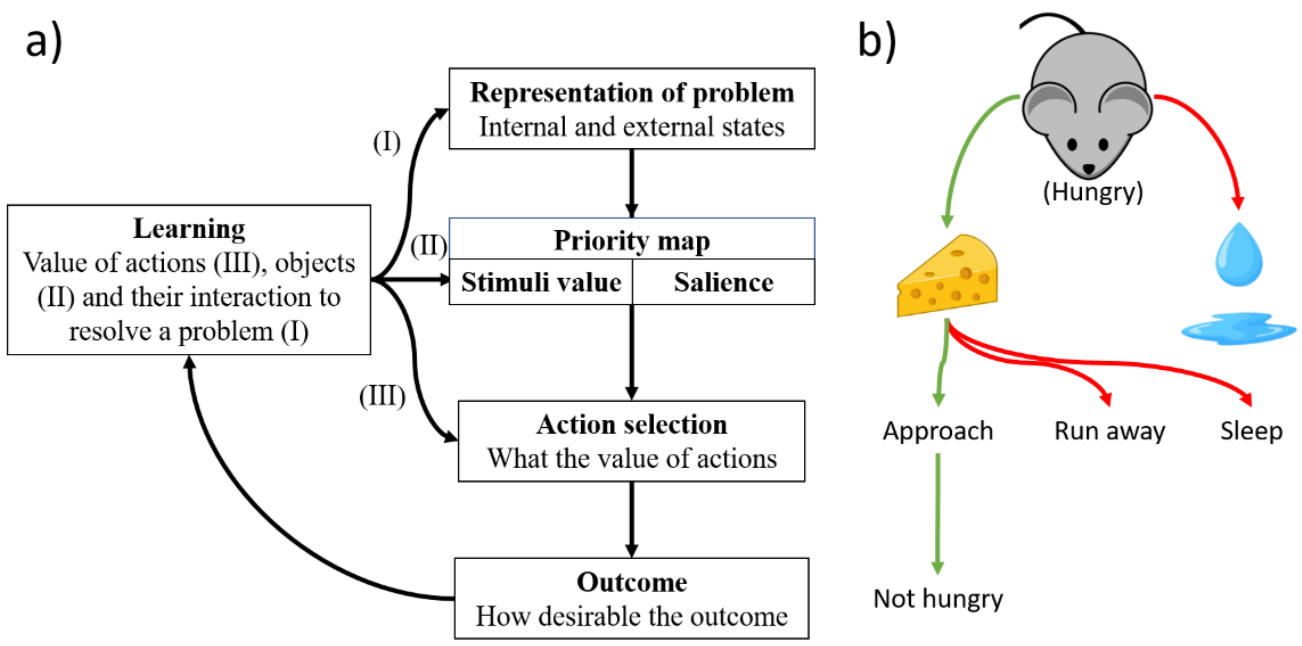

Figure 1 General concept of value-based decision making. a) A schematic representation of five stages involved in value-based decision-making. The first process is the representation of the problem that reflects the transformation of internal or external states (such as hunger) into a generalized set of objects or actions. The second process is the evaluation of the available objects in the surrounding environment in terms of their reward value or perceptual salience, which determine their prioritization for information processing. The third process is a selection of appropriate actions that are instrumental in maximizing positive outcomes. The fourth process is the processing of the received outcome and how much it resolved the initial problem. Finally, a learning process compares the obtained outcomes against the expected outcomes (i.e., prediction error). The prediction error serves as a modulatory signal that is sent to each of the preceding processing stages, thereby refining the representation of expected values. b) Illustration of how these stages are reflected in an animal's behavior.

\subsubsection{Reward guides decisions: Processes involved in value-based decision making}

The literature on reward mechanisms converges on a model (Figure 1) with five individual stages (Herd et al., 2021; Hull, 1943; Rangel et al., 2008; Schultz, 2015). Each stage reflects a separate process during the value-based decision-making. In the first stage, an organism formalizes a representation of the problem created by the imbalance within internal or external states. Here the information from senses (e.g., neurons in the stomach when hungry) is translated into a physical category (e.g., food consumption). In the second stage, activated representations are applied to the external information from the environment, where every available object goes through a prioritization process. Relevant objects (e.g., bread) gain priority for processing resources and thus attract attention. The third stage is a selection of the action (e.g., approach food to eat) that is used to interact with an object. Multiple actions could be selected. However, here an organism evaluates and selects the most energy-efficient action. Forth, after an organism executes the action and interacts with the object, it evaluates the outcome. Here information from the first stage is updated. Positive changes of the initial problem are translated to benefits for the organism, whereas negative changes are costs. At the fifth stage, the result from the outcome evaluation stage helps a learning process through computing an error signal, i.e. the 
difference between the expected and the obtained outcomes. This information helps the system to refine the stored representations of the expected reward of environmental stimuli and is transmitted back to the previous stages to help learning. This way, actions and objects that are associated with more than expected reward outcomes are reinforced. Additionally, object-action pairings are categorically learned as entities leading to an optimal or suboptimal outcome, hence helping the organism to make better choices in the future.

\subsubsection{Reward affects multiple stages of information processing: from sensation to action}

As demonstrated above, reward information is an important determinant of valuebased decisions. Reward effects can be additionally categorized based on the level of information processing in the brain that is most affected by them. As such, there are three general levels where reward information exerts effects (Berridge, 2004; O'Doherty et al., 2017): (1) goal-directed control of behavior, (2) action selection, and (3) sensory processing. Although these stages have unique properties and dedicated neuronal correlates, they also closely interact with each other, as will be discussed below.

\subsubsection{Reward affects goal-directed behavior}

Control of goal-directed behavior by reward information refers to a class of behaviors that appear as a drive or motivation toward a specific reward outcome. In literature, this reward modulation is reflected in value-based decision-making tasks (VBDM), where participants have to choose one option out of two (or more) possible options, as shown in Figure 1 (O'Doherty et al., 2017; Rangel et al., 2008). In addition to guiding choices towards most valuable items, it has also been shown that reward overall improves the speed and accuracy of decisions as participants select objects with higher reward outcomes faster and more accurately (Anderson et al., 2011a; Hickey et al., 2010a; Kiss et al., 2009; Stanisor et al., 2013). Additionally, an expectation of reward outcomes "energizes" behavior and modulates the effort towards rewarding objects (Pessiglione et al., 2018, 2007). Accordingly, theoretical frameworks suggest two distinct influences of reward on behavior (Dickinson and Balleine, 1994; Niv et al., 2006): goal-directed effects determine the current goal of behavior and affect behavior in an 'outcome-specific' manner. In contrast, 'energizing' effects of reward are more general and determine the vigor of all actions in an 'outcomeunspecific' and involuntary manner (Dickinson and Balleine, 1994; Niv et al., 2006). 


\subsubsection{Reward affects action selection}

Reward controls action selection. This mechanism of reward modulation has been studied in the context of value-based decision-making, as discussed before (see Figure 1), as well as in reward-dependent learning (Thorndike, 1911). Thorndike, in his experiments on operant conditioning, showed that by rewarding specific patterns of behavior, like pressing a lever by a cat, reward reinforces this behavior, and consequently, it increases the frequency and intensity of the rewarded actions (Berridge, 2000; Schultz, 2006). This type of learning is called reinforcement learning (RL), where each action is evaluated and executed in order to maximize possible outcomes (Sutton and Barto, 2018). The reinforcement of actions helps to optimize complex behavioral patterns by maximizing the outcome (Berridge, 2004; Friston, 2010) and minimizing actions that exploit available energy resources (Otto and Daw, 2019). However, these benefits come at a cost. Specifically, reinforced actions do not involve anticipation of reward (Thorndike, 1911). Thus, behavior becomes habitual, making it difficult to adjust according to the changes in the environment.

\subsubsection{Reward affects sensory processing of the reward-related stimuli}

In addition to affecting the behavioral output such as choices or actions, reward modulates the representation of sensory processing of surrounding stimuli if they consistently predict rewarding outcomes. The most striking instance of modulation of sensation by reward was first reported by Pavlov (1927) with his experiments on classical conditioning. He showed that a previously non-rewarding cue like a bell (conditioned stimuli $\mathrm{CS}$ ) produces an identical physiological (salvation) response as the primary reward cue like food (unconditioned stimulus US). In terms of reinforcement learning, the consistent delivery of reward with the CS reinforces the sensory representations of the non-reward CS stimulus (see also Figure 2). However, many decades after Pavlov's observations, recent studies started to shed light on the neural mechanisms of reward-driven modulation of sensory processing (Schultz, 1998; Schultz et al., 1997). Furthermore, recent studies have consistently shown that the earliest stages of sensory processing in the brain are modulated by information related to the reward value of stimuli (Arsenault et al., 2013; Schultz, 2015; Schultz et al., 1997; Serences, 2008; Shuler and Bear, 2006). It has remained unknown how specific sensory properties of stimuli, such as their spatial and temporal properties, sensory modality, and mode of attentional selection, interact with the reward-driven modulation of sensory processing. The focus of the current thesis is to shed light on this open question. 


\subsubsection{Neuronal mechanisms of reward effects on sensory perception}

\subsubsection{Reward modulation via Dopaminergic neurons during associative learning}

Following studies extended previous results and showed that reward prediction seems to rely on the activity of the ventral tegmental area (VTA) and substantia nigra (SN), specifically on the neurotransmitter dopamine (DA) (Schultz, 1998; Schultz et al., 1997). DA neurotransmitter influences receptors in other channels visual (Arsenault et al., 2013) and auditory (Bao et al., 2001) primary sensory areas, which makes it an important mechanism to control many physiological processes, including cognitive control (Westbrook et al., 2021) and reward processing (Schultz, 1998). Importantly, it has been shown that the activity of DA neurons is tightly linked in time with the onset of reward delivery as shown by direct recording from SN neurons (Schultz, 1986; Schultz et al., 1997) (Figure 2 Top). Specifically, it was shown that as learning of stimulus-reward associations progresses, the increase of activity of DA neurons could be shifted to the onset of the conditioned stimulus (CS), while the activity during the reward delivery stays at the baseline level (Figure 2 Middle). However, when reward delivery is withdrawn, the activity of DA neurons during reward delivery decreases to below the baseline level (Figure 2 Bottom). These findings show that reward could affect the sensory representation of a conditioned stimulus (CS) that occurs together with it. Critically, this reward modulation is persistent and continues even without reward delivery (Steinberg et al., 2013; Wise, 2004). 


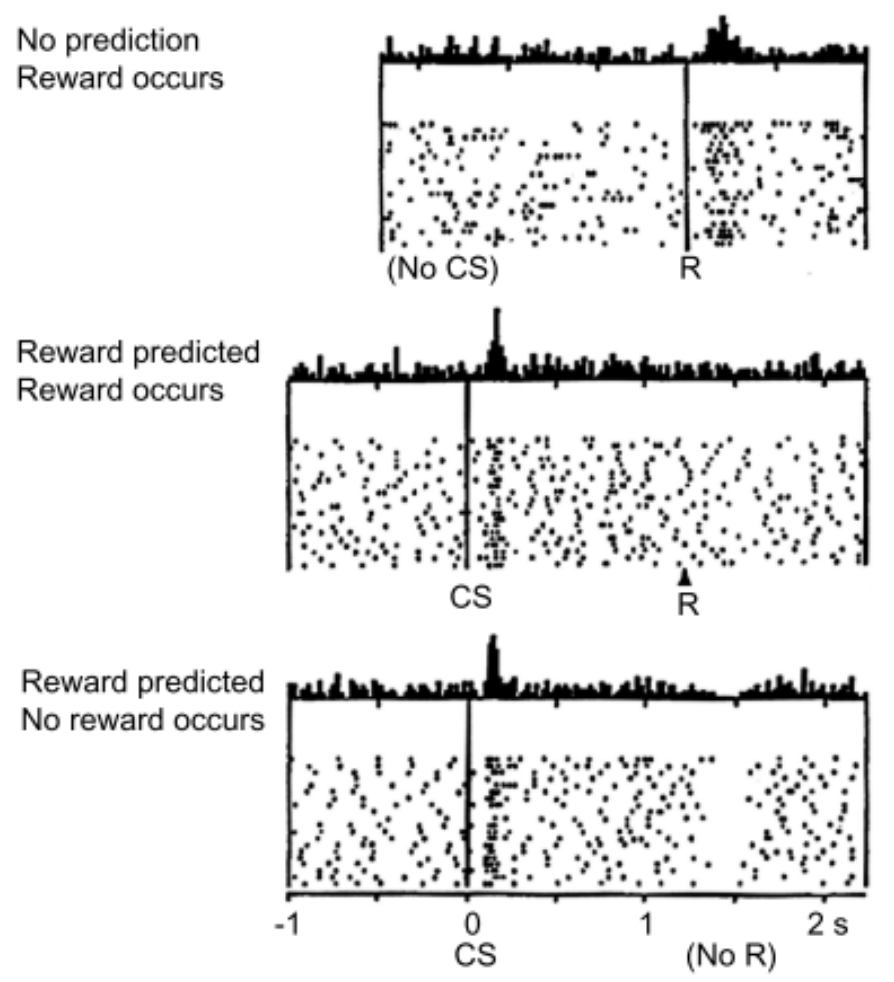

Figure 2. Dopamine neurons reflect reward prediction error. Raster plots with responses of a dopaminergic neuron in ventral tegmental area (VTA) during Pavlovian conditioning. (Top) The response of dopamine neurons at the onset of the unconditioned stimuli (US) with primary reward (R: food). (Middle) With progression of learning, the activity of dopamine neurons after the conditional stimulus (CS: e.g. a bell predicting food) increases, whereas at the same time, activity after US returns to the baseline. (Bottom) After conditioning, withdrawing the US with a reward (No R) suppresses the activity of dopamine neurons below the baseline during the period of reward delivery. Figure from (Schultz et al., 1997).

DA receptors are widely distributed in central and peripheral nervous systems (Arsenault et al., 2013; Bao et al., 2001; Lockhofen and Mulert, 2021; Vitay and Hamker, 2007), which makes them an important mechanism in mediating neuroplasticity (Calabresi et al., 2007; Jay, 2003; Schultz, 2002). For example, pairing a sound frequency with a rewarding outcome is followed by a DA release in the auditory cortex (Bao et al., 2001), which results in the long-term remodeling of the tuning curves in the respective neurons (A1). Similar effects were shown in the visual modality (Arsenault et al., 2013; Serences, 2008; Serences and Saproo, 2010; Shuler and Bear, 2006), where reward associated stimuli evoke a stronger neuronal response in primary visual areas as well as increase accuracy and response time to these stimuli.

1.1.5.2 Reward-driven modulation of sensory perception through attentional mechanisms and other types of top-down mechanisms

DA plays an important role in reward modulation (Pessiglione et al., 2006; Schultz, 2013, 2002, 1998) but is also involved in other functions, especially in attentional control (Floresco and Magyar, 2006; Westbrook et al., 2021). Thus, it is not surprising that often effects of reward modulation are interconnected with attentional mechanisms (Maunsell, 
2004). For example, Mogami and Tanaka (2006) showed that reward, associated with a stimulus, affects sensory processing of this stimulus proportionally to the amount of its value and that this modulation is reflected in the activity of dopaminergic neurons (for review see: Vitay and Hamker, 2007). According to the "incentive salience" model (Berridge and Robinson, 1998), a change in sensory processing should reflect a change in stimulus salience mediated by the activity of DA neurons. Consistent with this model, it was found that reward-driven modulations are accompanied by enhanced activity in attentional networks and attention-related DA release (Engelmann et al., 2009). At the behavioral level, reward improved performance and increased the signal-to-noise ratio of reward-related stimulus (Engelmann et al., 2009; Pessoa, 2015). These findings indicate that dopaminergic modulation is a powerful mechanism that improves attention control by improving sensory representation of the reward-related stimulus (Failing and Theeuwes, 2018).

In addition to the attentional mechanisms, there is evidence that value-related signals from the valuation network encompassing ventral striatum and orbitofrontal cortex (Rangel et al., 2008; Schultz, 2000) are fed back to early sensory areas. Such signals help to change the sensory representation of reward-related stimuli in the face of dynamic changes in the environment and/or reward associations (Cox et al., 2005; D. Liu et al., 2020). Interestingly, in addition to the classical reward network, recent studies have shown that the effect of reward on the primary visual cortex might be mediated through other sensory association areas. For instance, in a task where auditory reward value was shown to impact visual perception, multisensory association areas of the temporal cortex were also modulated by sound values suggesting that they may serve as an intermediary stage to better coordinate the interaction between the primary sensory cortices when high-value stimuli were presented (Pooresmaeili et al., 2014). This observation was a motivation for one of the objectives of the current thesis, i.e., to compare the cross-modal and intra-modal reward modulation which may or may not rely on such an intermediate stage of processing (see Chapter 2 and 3).

\subsubsection{Reward-driven modulation of perception through enhancing the plasticity of sensory areas}

Reward signals affect sensory perception even when the delivery of reward is halted, hence during a so-called no-reward phase (Anderson et al., 2013; Failing and Theeuwes, 2015; Maclean and Giesbrecht, 2015; Mine and Saiki, 2015; Rutherford et al., 2010; Watson et al., 2019). To explain reward effects that persist even when reward is not delivered anymore, a new framework referred to as 'reward history' has been suggested by recent studies (Awh et al., 2012; Failing and Theeuwes, 2018; Maclean and Giesbrecht, 2015; 
Theeuwes, 2019), which operates along with dopaminergic and/or top-down feedback mechanisms (Anderson, 2019). In this view, a prolonged association of a stimulus with reward and its accompanying dopaminergic reinforcement of reward predicting cues (i.e., CS) leads to long-term plasticity of sensory areas that encode the CS. The enhanced local plasticity of early sensory areas allows the persistent prioritized processing of rewardpredicting cues even when the reward delivery is halted (Anderson, 2017).

\subsubsection{Cognitive models of the interaction between reward and attention}

As outlined above, reward effects on sensory perception could be mediated through the engagement of attention (Chelazzi et al., 2013; Failing and Theeuwes, 2018; Pessoa, 2015). In fact, the interaction of reward and attention is so strong that their neural correlates are in some instances indistinguishable from one another (Maunsell, 2004), hence complicating their dissociation. Given this debate in the field, it is important to understand whether and how reward-driven modulation of perception can be explained in light of influential models of attention. We will therefore discuss these models in the following sections.

\subsubsection{General characteristic of Attentional mechanisms}

Attentional selection of sensory processing is guided by exogenous or endogenous factors (Carrasco, 2011). Exogenous factors are related to the stimulus, bottom-up salience, i.e., the degree to which a stimulus stands out from its surrounding. For example, a bright stimulus will have higher priority during visual processing than a dim stimulus simply by the difference in intensity of the signal. Prioritized processing is reflected in the change of neuronal response and yields to a measurable change in behavioral responses like response time and accuracy. Endogenous factors are determined by the goal-directed relevance of a specific type of information according to the demands of the task at hand. Furthermore, attentional mechanisms are commonly categorized based on the type of information that guides the selection of relevant information. Accordingly, three categories have been described: space-based attention, object-based attention, and feature-based attention (Carrasco, 2011). Space-based attention follows the traditional metaphor of "spotlight" (Hoffman and Nelson, 1981; Posner, 1980). A stimulus that falls under the spatial window of "spotlight" is enhanced and selected for further processing (Eriksen and Eriksen, 1974). Object-based attention relies on the representational basis of attention by objects (Duncan, 1984; Rock and Gutman, 1981). In series of experiment Kahneman, Treisman, and Gibbs (1992) proposed a concept of an "object file" that reflect a representation an object, that is a 
result of the integration of features that share the location of temporal alignment with this object. Finally, the mechanism of feature-based attention, rather than biasing attentional resources to certain location or object, biases attention to a unique feature. This mechanism is demonstrated by enhanced sensory processing of the attended feature across the visual field (Corbetta et al., 1990; Serences et al., 2009).

Attentional modulation of sensory processing has been found at the earliest stages of sensory processing in visual areas (V1, V2, V4) (Motter, 1993; Reynolds and Chelazzi, 2004; Wild and Treue, 2021) and overall also involve the frontoparietal attentional network that sends guiding feedback signals to early sensory areas (Corbetta and Shulman, 2002).

\subsubsection{Attentional priority map}

To discuss relations between reward and attentional mechanisms (Bourgeois et al., 2016; Failing and Theeuwes, 2018; Maunsell, 2004; Pessoa, 2015), it is important to have a computational model that reflects these relations. A good candidate is a priority map model. A priority map is a two-dimensional representation of the sensory information (predominantly visual) that reflects objects' attentional priorities within a determined space (Bisley, 2011; Itti and Koch, 2001; Wolfe, 2021). These attentional priorities integrate sensory properties of the external stimulus (bottom-up) and internal goals like the relevance and importance of the stimulus for the current task (top-down) (Trappenberg et al., 2001; Van Zoest et al., 2004). According to this framework, at first sensory inputs will indiscriminately activate all locations of such a map and a high priority will be given to physically salient stimuli irrespective of their relevance to the task, e.g., whether it is a target or a distractor. This is followed by a top-down selection process that adjusts the priority of stimuli according to the current goals. As such, a salient distractor will impair the task performance if the top-down control is weak or if the task is too difficult. This model is primarily based on the results on the visual search tasks (Wolfe, 2021), where at any given time, an observer first evaluates location values in the priority map and then makes a saccade to a location with the highest importance. The priority map model has been supported by neurophysiological studies showing that it is likely to be implemented at the lateral interparietal cortex (LIP), an area involved in planning of eye movements (Balan and Gottlieb, 2006), attentional selection (Corbetta and Shulman, 2002), and more recently in value-based decision making (Platt and Glimcher, 1999). 
a)

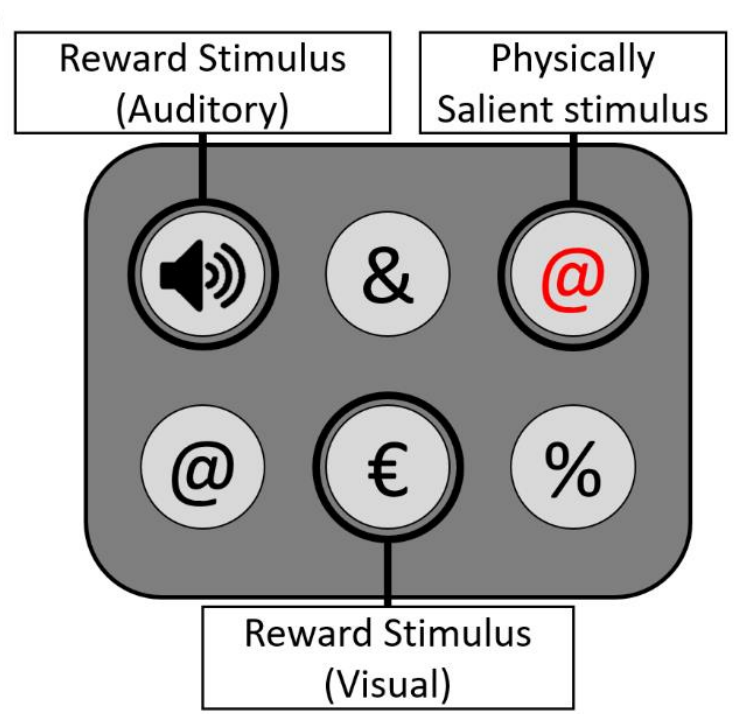

b)

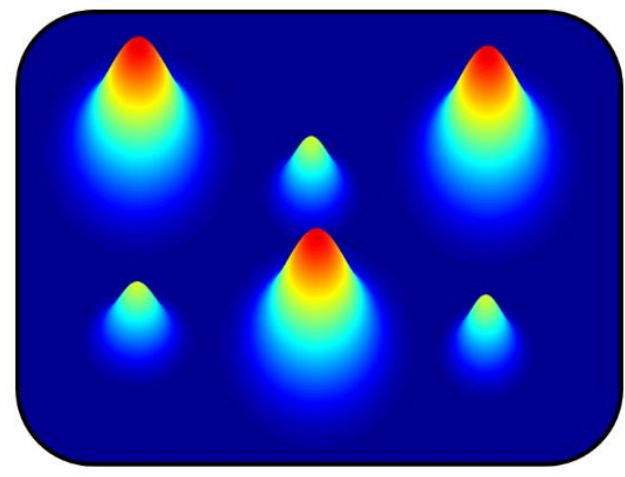

Figure 3. Embedding rewards into a priority map model of information processing. Priority map (Bisley, 2011) is a conceptual framework that explains how information is prioritized for further processing, especially through the allocation of attention. Although this framework is often in context of visual search tasks, the same conceptual framework can be applied to prioritization of information in other dimensions. a) an example of a hypothetical search task, where the task is to move eyes to the @ sign, i.e. the target. A physically salient distractor (top-right), a high-reward auditory stimulus (top-left) and a high-reward visual stimulus (bottommiddle) may all have an enhanced representation in such a map and hence influence the search task. b) Representation of each object in a on a putative priority map. Rewarding visual and auditory stimuli, as well as the physically salient distractor have a higher peak compared to other objects. The figure represents the current understanding of the reward mechanisms (Anderson et al., 2021; Failing and Theeuwes, 2018). It has remained unknown how reward value that is signaled through modalities other than vision can influence representations on such a map and how reward value and attention interact in such contexts.

\subsubsection{Reward guides the selective processing of representations on the priority map}

As mentioned above, during the processing of incoming sensory information at first, a high priority is given to physically salient stimuli, followed by selection mechanisms that adjust the priorities according to the goals of the tasks and other top-down factors. In this view, reward could boost stimulus representations on a priority map in three ways. Firstly, reward may enhance the bottom-up salience of the sensory stimuli (Anderson et al., 2011a; Hickey et al., 2010b; Serences, 2008; Theeuwes and Belopolsky, 2012; Wang et al., 2013). Secondly, reward-predicting cues can boost the sensory representations that lead to a reward in a top-down, goal-driven manner (Berridge, 2004; Berridge and Kringelbach, 2015). Recently, a new third category of reward-driven effects was identified (Awh et al., 2012). In this view, associating a stimulus with reward outcomes leads to prioritized processing through a mechanism called "reward selection history". The specific feature of this type of modulation is that through learning stimuli that were consistently selected in the past due to their value or importance receive additional prioritization in future that is above and beyond top-down and bottom-up factors (Failing and Theeuwes, 2017; Theeuwes, 2018). Against this background, the current thesis tries to shed light on interactions of reward and attention 
and hopefully provide a mechanistic understanding they should be included in influential cognitive models such as the priority map model.

\subsubsection{Neurophysiological signatures of reward-driven modulations}

Previous research has identified value-driven modulations of neuronal responses using various methods. Single-cell studies have shown reward-driven modulation of visual processing as early as in area V1 (Henschke et al., 2020; Shuler and Bear, 2006; Stanisor et al., 2013) and even in subcortical areas (Takakuwa et al., 2017). Electroencephalography (EEG) studies showed early modulations of the visual Event-Related Potentials (ERPs), including P1 (80-120 ms) (Bayer et al., 2019, 2012; Hammerschmidt et al., 2018) N1 (140$220 \mathrm{~ms}$ ) (Luque et al., 2017) and C1 ( 70 ms) (Bayer et al., 2017; Rossi et al., 2017). P1 and especially C1 ERP components are likely to originate from the primate and extrastriate visual cortices (Tankelevitch et al., 2020). Early mechanisms of reward modulation were also corroborated by fMRI studies. For instance, in visual areas V1, V2, V4 (Arsenault et al., 2013; Serences, 2008; Serences and Saproo, 2010; Shuler and Bear, 2006).

The current thesis uses the high temporal resolution of the EEG data to delineate different stages of reward modulation of stimulus processing across time.

\subsubsection{Measuring reward effects across sensory modalities}

Up to this point, we discussed early reward effects from the perspective of the visual system. However, reward mechanisms are also present in other sensory modalities, as shown by recent studies (Goldstein et al., 2006; Pleger et al., 2008; Rutkowski and Weinberger, 2005; Weil et al., 2010). Thus, it is important to understand if reward mechanisms reflect a broad principle of information processing across sensory modalities and how reward information is communicated across senses. For instance, recent evidence showed that auditory reward stimuli could influence sensory processing in the visual modality (Anderson, 2016a; Leo and Noppeney, 2014; Pooresmaeili et al., 2014; Sanz et al., 2018). Notably, auditory reward cues in these studies were task-irrelevant, but instead of acting as distractors that capture attention away from the target, led to a facilitation in target processing (Leo and Noppeney, 2014; Pooresmaeili et al., 2014). This is in contrast to the commonly reported interference effect of the high-value intra-modal distractors (Yantis et al., 2012) and points to a potential difference between intra-modal and cross-modal reward effects. On the other hand, another possibility is that cross-modal and intra-modal reward modulations have similar effects as reward cues from different modalities exert their effect 
in a supra-modal manner that is independent of sensory modality. However, cross-modal and intra-modal reward effects have not been directly compared against each other.

Understanding cross-modal reward effects also advances our understanding of basic mechanisms of integration of signals across sensory modalities. Previous studies have provided numerous examples of interaction between senses. For instance, it has been shown that pairing auditory and visual stimuli can decrease response time (Shams and Seitz, 2008) and increase accuracy (Diederich and Colonius, 1987; Ngo and Spence, 2010; Seitz et al., 2006; Von Kriegstein and Giraud, 2006) compared to uni-sensory stimuli. In other studies, multisensory stimulus showed an advantage in sensory processing of its unimodal components. This was measured using d' (sensitivity measurement) measurement, where concurrent irrelevant sound improved the visual sensitivity of target stimuli (Bolognini et al., 2005). Effects of multisensory integration on d' indicate that these effects not only influence behavioral responses but also play a role in early mechanisms of sensory processing by tuning the response of neurons involved in the processing of this multisensory stimulus (Frassinetti et al., 2002). However, the interaction between sensory and value crossmodal integration remains unknown.

Taken together, measuring reward effects across sensory modalities might be a powerful tool to understand mechanisms of reward modulation in particular and the basics of sensory processing in general. 


\subsection{Objectives and structure of the current thesis}

\subsubsection{Summary of our current knowledge regarding reward effects on sensory perception}

As discussed in previous sections, reward is a powerful mechanism that guides our decisions, actions and even affects early stimuli sensory representations (Anderson, 2013; Hickey et al., 2010b; Serences, 2008). With the reward-driven modulation of perception, subtle yet valuable signals gain processing priority, resulting in faster detection and more efficient consumption behavior, which is essential for our well-being. Recent findings reported that reward signals could influence automatically and involuntarily even when reward signals are irrelevant for the current goals (Anderson, 2013; Anderson et al., 2011a; Hickey et al., 2010b; Pessoa and Engelmann, 2010; Pleger et al., 2008). There is an ongoing debate on whether attentional and reward mechanisms are independent (Maunsell, 2004; Pessoa, 2015), and recent studies suggested that reward may elicit an independent mechanism by itself that is referred to a reward selection history, which operates parallel to goal-driven and stimulus-driven attentional mechanisms (Awh et al., 2012; Failing and Theeuwes, 2018; Maclean and Giesbrecht, 2015; Theeuwes, 2019). Neurophysiological studies revealed that such reward modulations could affect information processing in early primary visual areas (Arsenault et al., 2013; Serences, 2008) and could be found as early as C1 (60-90 ms) and P1 (90-120 ms) ERP components (Bayer et al., 2017; Hickey et al., 2010b; Luque et al., 2017). Notably, in natural environments, reward predictions are often based on information from multiple sensory modalities (Wise, 2004). Thus, it is important to understand how reward information is transferred across different sensory modalities. Indeed, previous findings showed that reward signals help to integrate information between modalities (Bean et al., 2021; Bruns et al., 2014; Cheng et al., 2020; Sanz et al., 2018) and can modulate the sensory representation of stimuli in another sensory modality (Anderson, 2016a; Cheng et al., 2020; Pooresmaeili et al., 2014).

\subsubsection{Open questions}

The majority of previous research has been focused on the effect of reward on visual perception, mediated through reward cues that are also presented visually. Recent studies have reported reward modulations in other sensory modalities other than vision and effects that occur cross-modally. Yet, it is unknown whether intra-modal and cross-modal reward effects exert similar effects on sensory processing. Results from previous findings indicate that cross-modal reward signals could both enhance processing of sensory representation of the target stimuli (Pooresmaeili et al., 2014) as well as suppress it since the reward stimulus 
is task-irrelevant (Anderson, 2016a). Similarly, both facilitatory, as well as suppressive effects have been reported for intra-modal cues, depending on whether reward cue is the target or the distractor of the task. Therefore, to gain a better insight into the underlying mechanisms of reward modulations across sensory modalities, it is important to compare intra-modal and cross-modal rewards directly and within the same task while neurophysiological data is concurrently recorded.

It is also important to evaluate how selective attention and reward interact. Current scientific evidence cannot disentangle reward and attentional mechanisms (Maunsell, 2004). In most cases, reward value is induced by an attentional task, and attention is controlled by reward delivery (but see: Baldassi and Simoncini, 2011). By contrasting effects of cross- and intra-modal reward cues, we will identify whether attention and reward interact in a modality-specific manner or whether general principles govern their interactions.

\subsubsection{Objectives}

The overarching goal of this doctoral thesis is to gain insight into the underlying mechanisms of reward-driven modulation of sensory perception. To this end, we seek to realize two key objectives:

- Objective 1: Understanding how intra- and cross-modal reward cues influence early sensory processing.

- Objective 2: Understanding how intra- and cross-modal rewards interact with attention.

To achieve these objectives, we conducted three studies. In all studies, participants first learned the reward associations of visual and auditory cues and then were tested during a no-reward phase, when cues were not predictive of the reward delivery but could affect perception due to their persistent privileged processing.

The first study (Chapter 2) focused on the electrophysiological signatures of intraand cross-modal reward modulation (Objective 1). Previous findings have shown a wide range of reward effects across different stages of sensory processing (Bachman et al., 2020; Baines et al., 2011; Bayer et al., 2017; Hickey et al., 2010b; Luque et al., 2017; Novak and Foti, 2015; Pfabigan et al., 2014). However, despite reward effects found in the early stages of sensory processing across different sensory modalities, intra- and cross-modal reward effects on sensory processing were not directly compared. Thus, the first study aimed to compare intra-modal (visual) and cross-modal (auditory) reward cues on visual sensitivity. For this, we adapted an existing experimental paradigm with an orientation discrimination task in the presence of task-irrelevant cues that were previously associated with reward 
(Pooresmaeili et al., 2014). Based on previous literature, we predicted that the reward modulations should be in the same direction, i.e., facilitatory, in both cross- and intra-modal rewards. Additionally, we predicted two possible scenarios regarding the timing of reward effects based on previous studies: 1) the computation of reward value occurs in parallel with early sensory processing within each sensory modality and hence happens at the same time in both intra-modal and cross-modal rewards; 2) reward signals emerge at the later stages of sensory processing where information from different sensory modalities is combined, and hence cross-modal reward effects occur later than intra-modal effects. To explore these cases, we focused on the early ERP components like P1 (70-170 ms) and N1 (180-250 ms). The procedures of this study were preregistered (https://osf.io/47 wxr/).

In the second study (Chapter 3), we wanted to further characterize similarities and differences of cross- and intra-modal reward cues in relation to their spatiotemporal characteristics (Objective 1). Specifically, we compared the cross-modal and intra-modal reward effects in terms of their optimal size of peri-stimulus attentional spotlight and timing of their presentation relative to the target. To this end, we used an experimental paradigm similar to the first study with intra- and cross-modal reward cues, and at the same time, we controlled the spatial and temporal arrangement of the reward cues relative to the target. This study measured behavioral indices of reward modulation (visual sensitivity and reaction times).

In our third study (Chapter 4), we aimed to shed light on the interaction between reward and attention and its dependence on rewards' sensory modality (Objective 2). To this end, we adapted the design of a previous study (Talsma and Woldorff, 2005) where participants detected a brief change in an audio-visual stimulus. Importantly, we manipulated both the locus of spatial attention and the reward value associated with the audiovisual stimuli. Participants were required to only report changes occurring on the attended side (varied across blocks). Both, only one, or none of the modalities were associated with high reward. This design allowed us to examine the interaction of spatial attention and reward value signaled through different sensory modalities. We hypothesized that reward and attention independently affect behavioral and electrophysiological (i.e., visual P1 and auditory N1 ERP components) correlates of sensory processing, with both enhancing perception. Additionally, we hypothesized that the reward-driven modulations of ERPs depended on rewards' sensory modality, with maximal modulation of visual ERPs expected to occur for visual high reward cues, and maximal modulation of auditory ERPS expected to occur for auditory high reward cues. This allowed us to investigate how reward guides 
the allocation of attention and how, on the other hand, reward effects are modulated by the allocation of attention across different sensory modalities. The procedures of this study were preregistered (https://osf.io/xte4v).

With these studies, the current thesis seeks three long-term goals. Firstly, this thesis will pave the way to identify the biological markers of reward-driven effects within and across sensory modalities. Secondly, a deeper understanding of biological mechanisms of cross- and intra-modal reward modulation will allow the refinement of computational and cognitive models of sensory processing such as a putative priority map illustrated above. Lastly, a deeper understanding of neuronal mechanisms behind cross- and intra-modal reward modulation can help to develop better rehabilitation procedures for patients with impairments in one sensory modality. These long-term goals, as well as the general conclusions driven from the three studies reported in this thesis, will be further discussed in

\section{Chapter 5.}




\subsection{References}

Ainslie, G., 1975. Specious reward: A behavioral theory of impulsiveness and impulse control. Psychol. Bull. 82, 463-496. https://doi.org/10.1037/h0076860

Alais, D., Morrone, C., Burr, D., 2006. Separate attentional resources for vision and audition. Proc. R. Soc. B Biol. Sci. 273, 1339-1345. https://doi.org/10.1098/rspb.2005.3420

Anderson, B.A., 2019. Neurobiology of value-driven attention. Curr. Opin. Psychol. https://doi.org/10.1016/j.copsyc.2018.11.004

Anderson, B.A., 2017. Reward processing in the value-driven attention network: reward signals tracking cue identity and location. Soc. Cogn. Affect. Neurosci. 12, 461-467. https://doi.org/10.1093/scan/nsw141

Anderson, B.A., 2016. Value-driven attentional capture in the auditory domain. Attention, Perception, Psychophys. 78, 242-250. https://doi.org/10.3758/s13414-015-1001-7

Anderson, B.A., 2013. A value-driven mechanism of attentional selection. J. Vis. 13, 7-7. https://doi.org/10.1167/13.3.7

Anderson, B.A., Kim, H., Kim, A.J., Liao, M.-R., Mrkonja, L., Clement, A., Grégoire, L., 2021. The Past, Present, and Future of Selection History. Neurosci. Biobehav. Rev. 130, 326-350. https://doi.org/10.1016/j.neubiorev.2021.09.004

Anderson, B.A., Laurent, P.A., Yantis, S., 2011. Value-driven attentional capture. Proc. Natl. Acad. Sci. U. S. A. 108, 10367-10371. https://doi.org/10.1073/pnas.1104047108

Anderson, Laurent, Yantis, Anderson, B.A., Laurent, P.A., Yantis, S., 2013. Reward predictions bias attentional selection. Front. Hum. Neurosci. 7, 1-6. https://doi.org/10.3389/fnhum.2013.00262

Arsenault, J.T., Nelissen, K., Jarraya, B., Vanduffel, W., 2013. Dopaminergic Reward Signals Selectively Decrease fMRI Activity in Primate Visual Cortex. Neuron 77, 1174-1186. https://doi.org/10.1016/j.neuron.2013.01.008

Awh, E., Belopolsky, A. V., Theeuwes, J., 2012. Top-down versus bottom-up attentional control: A failed theoretical dichotomy. Trends Cogn. Sci. https://doi.org/10.1016/j.tics.2012.06.010

Bachman, M.D., Wang, L., Gamble, M.L., Woldorff, M.G., 2020. Physical Salience and ValueDriven Salience Operate through Different Neural Mechanisms to Enhance Attentional Selection. J. Neurosci. 40, 5455-5464. https://doi.org/10.1523/JNEUROSCI.1198-19.2020

Baines, S., Ruz, M., Rao, A., Denison, R., Nobre, A.C., 2011. Modulation of neural activity by motivational and spatial biases. Neuropsychologia 49, 2489-2497. https://doi.org/10.1016/j.neuropsychologia.2011.04.029

Balan, P.F., Gottlieb, J., 2006. Integration of exogenous input into a dynamic salience map revealed by perturbing attention. J. Neurosci. 26, 9239-9249. https://doi.org/10.1523/JNEUROSCI.1898-06.2006

Baldassi, S., Simoncini, C., 2011. Reward sharpens orientation coding independently of attention. Front. Neurosci. 5, 13. https://doi.org/10.3389/fnins.2011.00013

Bao, S., Chan, V.T., Merzenich, M.M., 2001. Cortical remodelling induced by activity of ventral tegmental dopamine neurons. Nature 412, 79-83. https://doi.org/10.1038/35083586

Basten, U., Biele, G., Heekeren, H.R., Fiebach, C.J., 2010. How the brain integrates costs and benefits during decision making. Proc. Natl. Acad. Sci. U. S. A. 107, 21767-21772. https://doi.org/10.1073/pnas.0908104107

Bayer, M., Grass, A., Schacht, A., 2019. Associated valence impacts early visual processing of letter strings: Evidence from ERPs in a cross-modal learning paradigm. Cogn. Affect. Behav. Neurosci. 19, 98-108. https://doi.org/10.3758/s13415-018-00647-2

Bayer, M., Rossi, V., Vanlessen, N., Grass, A., Schacht, A., Pourtois, G., 2017. Independent effects of motivation and spatial attention in the human visual cortex. Soc. Cogn. Affect. Neurosci. 12, 146-156. https://doi.org/10.1093/scan/nsw162

Bayer, M., Sommer, W., Schacht, A., 2012. P1 and beyond: Functional separation of multiple emotion effects in word recognition. Psychophysiology 49, 959-969. https://doi.org/10.1111/j.1469-8986.2012.01381.x

Bean, N.L., Stein, B.E., Rowland, B.A., 2021. Stimulus value gates multisensory integration. Eur. J. Neurosci. 53, 3142-3159. https://doi.org/10.1111/ejn.15167 
Berridge, K.C., 2004. Motivation concepts in behavioral neuroscience. Physiol. Behav. 81, 179-209. https://doi.org/10.1016/J.PHYSBEH.2004.02.004

Berridge, K.C., 2000. Reward learning: Reinforcement, incentives, and expectations. Psychol. Learn. Motiv. - Adv. Res. Theory 40, 223-278.

Berridge, K.C., Kringelbach, M.L., 2015. Pleasure Systems in the Brain. Neuron 86, 646-664. https://doi.org/10.1016/J.NEURON.2015.02.018

Berridge, K.C., Robinson, T.E., 1998. What is the role of dopamine in reward: Hedonic impact, reward learning, or incentive salience? Brain Res. Rev. 28, 309-369. https://doi.org/10.1016/S0165-0173(98)00019-8

Bisley, J.W., 2011. The neural basis of visual attention. J. Physiol. https://doi.org/10.1113/jphysiol.2010.192666

Bolognini, N., Frassinetti, F., Serino, A., Làdavas, E., 2005. “Acoustical vision” of below threshold stimuli: Interaction among spatially converging audiovisual inputs. Exp. Brain Res. 160, $273-$ 282. https://doi.org/10.1007/s00221-004-2005-Z

Bourgeois, A., Chelazzi, L., Vuilleumier, P., 2016. How motivation and reward learning modulate selective attention. Prog. Brain Res. 229, 325-342. https://doi.org/10.1016/BS.PBR.2016.06.004

Braver, T.S., Krug, M.K., Chiew, K.S., Kool, W., Andrew Westbrook, J., Clement, N.J., Alison Adcock, R., Barch, D.M., Botvinick, M.M., Carver, C.S., Cools, R., Custers, R., Dickinson, A., Dweck, C.S., Fishbach, A., Gollwitzer, P.M., Hess, T.M., Isaacowitz, D.M., Mather, M., Murayama, K., Pessoa, L., Samanez-Larkin, G.R., Somerville, L.H., 2014. Mechanisms of motivation-cognition interaction: Challenges and opportunities. Cogn. Affect. Behav. Neurosci. https://doi.org/10.3758/s13415-014-0300-0

Bruns, P., Maiworm, M., Röder, B., 2014. Reward expectation influences audiovisual spatial integration. Attention, Perception, Psychophys. 76, 1815-1827. https://doi.org/10.3758/s13414-014-0699-y

Calabresi, P., Picconi, B., Tozzi, A., Di Filippo, M., 2007. Dopamine-mediated regulation of corticostriatal synaptic plasticity. Trends Neurosci. https://doi.org/10.1016/j.tins.2007.03.001

Carrasco, M., 2011. Visual attention: The past 25 years. Vision Res. 51, 1484-1525. https://doi.org/10.1016/J.VISRES.2011.04.012

Chelazzi, L., Perlato, A., Santandrea, E., Della Libera, C., 2013. Rewards teach visual selective attention. Vision Res. 85, 58-72. https://doi.org/10.1016/J.VISRES.2012.12.005

Cheng, Saglam, A., André, S., Pooresmaeili, A., 2020. Cross-Modal Integration of Reward Value during Oculomotor Planning. eneuro 7, ENEURO.0381-19.2020. https://doi.org/10.1523/ENEURO.0381-19.2020

Corbetta, M., Miezin, F., Dobmeyer, S., Shulman, G., Petersen, S., 1990. Attentional modulation of neural processing of shape, color, and velocity in humans. Science (80-. ). 248, 1556-1559. https://doi.org/10.1126/SCIENCE.2360050

Corbetta, M., Shulman, G.L., 2002. Control of goal-directed and stimulus-driven attention in the brain. Nat. Rev. Neurosci. 3, 201-215. https://doi.org/10.1038/nrn755

Cornelio, P., Velasco, C., Obrist, M., 2021. Multisensory Integration as per Technological Advances: A Review. Front. Neurosci. https://doi.org/10.3389/fnins.2021.652611

Cox, S.M.L., Andrade, A., Johnsrude, I.S., 2005. Learning to like: A role for human orbitofrontal cortex in conditioned reward. J. Neurosci. 25, 2733-2740. https://doi.org/10.1523/JNEUROSCI.3360-04.2005

Dawkins, R., 2016. The selfish gene.

Desimone, R., Duncan, J., 1995. NEURAL MECHANISMS OF SELECTIVE VISUAL ATTENTION, Annu. Rev. Neurosci.

Dickinson, A., Balleine, B., 1994. Motivational control of goal-directed action. Anim. Learn. Behav. 1994221 22, 1-18. https://doi.org/10.3758/BF03199951

Diederich, A., Colonius, H., 1987. Intersensory facilitation in the motor component? - A reaction time analysis. Psychol. Res. 49, 23-29. https://doi.org/10.1007/BF00309199

Duncan, J., 1984. Selective attention and the organization of visual information. J. Exp. Psychol. Gen. 113, 501-517. https://doi.org/10.1037/0096-3445.113.4.501

Engelmann, J.B., Damaraju, E., Padmala, S., Pessoa, L., 2009. Combined effects of attention and motivation on visual task performance: Transient and sustained motivational effects. Front. 
Hum. Neurosci. 3, 4. https://doi.org/10.3389/neuro.09.004.2009

Eriksen, B.A., Eriksen, C.W., 1974. Effects of noise letters upon the identification of a target letter in a nonsearch task. Percept. Psychophys. 1974 161 16, 143-149. https://doi.org/10.3758/BF03203267

Failing, M., Theeuwes, J., 2018. Selection history: How reward modulates selectivity of visual attention. Psychon. Bull. Rev. 25, 514-538. https://doi.org/10.3758/s13423-017-1380-y

Failing, M., Theeuwes, J., 2017. Don't let it distract you: how information about the availability of reward affects attentional selection. Attention, Perception, Psychophys. 79, 2275-2298. https://doi.org/10.3758/s13414-017-1376-8

Failing, M.F., Theeuwes, J., 2015. Nonspatial attentional capture by previously rewarded scene semantics. Vis. cogn. 23. https://doi.org/10.1080/13506285.2014.990546

Floresco, S.B., Magyar, O., 2006. Mesocortical dopamine modulation of executive functions: Beyond working memory. Psychopharmacology (Berl). https://doi.org/10.1007/s00213-0060404-5

Fox, C.R., Poldrack, R.A., 2009. Prospect theory and the brain, in: Neuroeconomics. Elsevier Inc., pp. 145-173. https://doi.org/10.1016/B978-0-12-374176-9.00011-7

Frassinetti, F., Bolognini, N., Làdavas, E., 2002. Enhancement of visual perception by crossmodal visuo-auditory interaction. Exp. Brain Res. 147, 332-343. https://doi.org/10.1007/s00221-002$1262-y$

Frederick, S., Loewenstein, G., O’Donoghue, T., 2002. Time Discounting and Time Preference: A Critical Review. J. Econ. Lit. 40, 351-401. https://doi.org/10.1257/002205102320161311

Frens, M.A., Van Opstal, A.J., Van Der Willigen, R.F., 1995. Spatial and temporal factors determine auditory-visual interactions in human saccadic eye movements. Percept. Psychophys. 57, 802816. https://doi.org/10.3758/BF03206796

Friston, K., 2010. The free-energy principle: a unified brain theory? Nat. Rev. Neurosci. 2010112 11, 127-138. https://doi.org/10.1038/nrn2787

Gold, J.I., Shadlen, M.N., 2007. The Neural Basis of Decision Making. Annu. Rev. Neurosci. 30, 535-574. https://doi.org/10.1146/annurev.neuro.29.051605.113038

Goldring, J.E., Dorris, M.C., Corneil, B.D., Ballantyne, P.A., Munoz, D.P., 1996. Combined eyehead gaze shifts to visual and auditory targets in humans. Exp. Brain Res. 111, 68-78. https://doi.org/10.1007/BF00229557

Goldstein, R.Z., Cottone, L.A., Jia, Z., Maloney, T., Volkow, N.D., Squires, N.K., 2006. The effect of graded monetary reward on cognitive event-related potentials and behavior in young healthy adults. Int. J. Psychophysiol. 62, 272-279. https://doi.org/10.1016/j.ijpsycho.2006.05.006

Hammerschmidt, W., Kagan, I., Kulke, L., Schacht, A., 2018. Implicit reward associations impact face processing: Time-resolved evidence from event-related brain potentials and pupil dilations. Neuroimage 179, 557-569. https://doi.org/10.1016/j.neuroimage.2018.06.055

Henschke, J.U., Dylda, E., Katsanevaki, D., Dupuy, N., Currie, S.P., Amvrosiadis, T., Pakan, J.M.P., Rochefort, N.L., 2020. Reward Association Enhances Stimulus-Specific Representations in Primary Visual Cortex. Curr. Biol. 30, 1866-1880.e5. https://doi.org/10.1016/j.cub.2020.03.018

Herd, S., Krueger, K., Nair, A., Mollick, J., O’Reilly, R., 2021. Neural Mechanisms of Human Decision-Making. Cogn. Affect. Behav. Neurosci. 21, 35-57. https://doi.org/10.3758/s13415020-00842-0

Hickey, C., Chelazzi, L., Theeuwes, J., 2010a. Reward guides vision when it's your thing: Trait reward-seeking in reward-mediated visual priming. PLoS One 5, 14087. https://doi.org/10.1371/journal.pone.0014087

Hickey, C., Chelazzi, L., Theeuwes, J., 2010b. Reward changes salience in human vision via the anterior cingulate. J. Neurosci. 30, 11096-11103. https://doi.org/10.1523/JNEUROSCI.102610.2010

Hoffman, J.E., Nelson, B., 1981. Spatial selectivity in visual search. Percept. Psychophys. 1981303 30, 283-290. https://doi.org/10.3758/BF03214284

Hull, C., 1943. Principles of behavior: An introduction to behavior theory. Appleton-Century-Crofts, New York.

Itti, L., Koch, C., 2001. Computational modelling of visual attention. Nat. Rev. Neurosci. 2, 194203. https://doi.org/10.1038/35058500 
Jay, T.M., 2003. Dopamine: A potential substrate for synaptic plasticity and memory mechanisms. Prog. Neurobiol. https://doi.org/10.1016/S0301-0082(03)00085-6

Kahneman, D., Treisman, A., Gibbs, B.J., 1992. The reviewing of object files: Object-specific integration of information. Cogn. Psychol. 24, 175-219. https://doi.org/10.1016/00100285(92)90007-O

Kahneman, D., Tversky, A., 2013. Choices, Values, and Frames. pp. 269-278. https://doi.org/10.1142/9789814417358_0016

Kiss, M., Driver, J., Eimer, M., 2009. Reward Priority of Visual Target Singletons Modulates EventRelated Potential Signatures of Attentional Selection. Psychol. Sci. 20, 245-251. https://doi.org/10.1111/j.1467-9280.2009.02281.x

Leo, F., Noppeney, U., 2014. Conditioned Sounds Enhance Visual Processing. PLoS One 9, e106860. https://doi.org/10.1371/journal.pone.0106860

Liu, D., Deng, J., Zhang, Z., Zhang, Z.Y., Sun, Y.G., Yang, T., Yao, H., 2020. Orbitofrontal control of visual cortex gain promotes visual associative learning. Nat. Commun. 11, 1-14. https://doi.org/10.1038/s41467-020-16609-7

Lockhofen, D.E.L., Mulert, C., 2021. Neurochemistry of Visual Attention. Front. Neurosci. 15, 517. https://doi.org/10.3389/fnins.2021.643597

Luque, D., Beesley, T., Morris, R.W., Jack, B.N., Griffiths, O., Whitford, T.J., Le Pelley, M.E., 2017. Goal-directed and habit-like modulations of stimulus processing during reinforcement learning. J. Neurosci. 37, 3009-3017. https://doi.org/10.1523/JNEUROSCI.3205-16.2017

Maclean, M.H., Giesbrecht, B., 2015. Neural evidence reveals the rapid effects of reward history on selective attention. Brain Res. 1606, 86-94. https://doi.org/10.1016/j.brainres.2015.02.016

Maunsell, J.H.R., 2004. Neuronal representations of cognitive state: Reward or attention? Trends Cogn. Sci. 8, 261-265. https://doi.org/10.1016/j.tics.2004.04.003

Meredith, M.A., Stein, B.E., 1986. Visual, auditory, and somatosensory convergence on cells in superior colliculus results in multisensory integration. J. Neurophysiol. 56, 640-662. https://doi.org/10.1152/jn.1986.56.3.640

Mine, C., Saiki, J., 2015. Task-irrelevant stimulus-reward association induces value-driven attentional capture. Attention, Perception, Psychophys. 77, 1896-1907. https://doi.org/10.3758/s13414-015-0894-5

Mogami, T., Tanaka, K., 2006. Reward association affects neuronal responses to visual stimuli in macaque TE and perirhinal cortices. J. Neurosci. 26, 6761-6770. https://doi.org/10.1523/JNEUROSCI.4924-05.2006

Motter, B.C., 1993. Focal attention produces spatially selective processing in visual cortical areas V1, V2, and V4 in the presence of competing stimuli. J. Neurophysiol. 70, 909-919. https://doi.org/10.1152/jn.1993.70.3.909

Nakayama, K., 1990. The iconic bottleneck and the tenuous link between early visual processing and perception, in: Blakemore, C. (Ed.), Vision: Coding and Efficiency. Cambridge: Cambridge University Press, pp. 411-422.

Ngo, M.K., Spence, C., 2010. Crossmodal facilitation of masked visual target identification. Attention, Perception, Psychophys. 72, 1938-1947. https://doi.org/10.3758/APP.72.7.1938

Niv, Y., Joel, D., Dayan, P., 2006. A normative perspective on motivation. Trends Cogn. Sci. 10, 375-381. https://doi.org/10.1016/J.TICS.2006.06.010

Novak, K.D., Foti, D., 2015. Teasing apart the anticipatory and consummatory processing of monetary incentives: An event-related potential study of reward dynamics. Psychophysiology 52, 1470-1482. https://doi.org/10.1111/psyp.12504

O'Doherty, J.P., Cockburn, J., Pauli, W.M., 2017. Learning, Reward, and Decision Making. Annu. Rev. Psychol. 68, 73-100. https://doi.org/10.1146/annurev-psych-010416-044216

Otto, A.R., Daw, N.D., 2019. The opportunity cost of time modulates cognitive effort. Neuropsychologia 123, 92-105. https://doi.org/10.1016/j.neuropsychologia.2018.05.006

Pavlov, 1927. Conditioned Reflexes. Oxford University Press, Oxford.

Pessiglione, M., Schmidt, L., Draganski, B., Kalisch, R., Lau, H., Dolan, R.J., Frith, C.D., 2007. How the Brain Translates Money into Force: A Neuroimaging Study of Subliminal Motivation. Science 316, 904. https://doi.org/10.1126/SCIENCE.1140459

Pessiglione, M., Seymour, B., Flandin, G., Dolan, R.J., Frith, C.D., 2006. Dopamine-dependent prediction errors underpin reward-seeking behaviour in humans. Nature 442, 1042-1045. 
https://doi.org/10.1038/nature05051

Pessiglione, M., Vinckier, F., Bouret, S., Daunizeau, J., Le Bouc, R., 2018. Why not try harder? Computational approach to motivation deficits in neuro-psychiatric diseases, Brain.

Pessoa, L., 2015. Multiple influences of reward on perception and attention. Vis. cogn. 23, 272-290. https://doi.org/10.1080/13506285.2014.974729

Pessoa, L., Engelmann, J.B., 2010. Embedding reward signals into perception and cognition. Front. Neurosci. 4, 17. https://doi.org/10.3389/fnins.2010.00017

Peters, J., Büchel, C., 2010. Neural representations of subjective reward value. Behav. Brain Res. 213, 135-141. https://doi.org/10.1016/J.BBR.2010.04.031

Pfabigan, D.M., Seidel, E.M., Sladky, R., Hahn, A., Paul, K., Grahl, A., Küblböck, M., Kraus, C., Hummer, A., Kranz, G.S., Windischberger, C., Lanzenberger, R., Lamm, C., 2014. P300 amplitude variation is related to ventral striatum BOLD response during gain and loss anticipation: An EEG and fMRI experiment. Neuroimage 96, 12-21. https://doi.org/10.1016/j.neuroimage.2014.03.077

Platt, M.L., Glimcher, P.W., 1999. Neural correlates of decision variables in parietal cortex. Nature 400, 233-238. https://doi.org/10.1038/22268

Pleger, B., Blankenburg, F., Ruff, C.C., Driver, J., Dolan, R.J., 2008. Reward facilitates tactile judgments and modulates hemodynamic responses in human primary somatosensory cortex. J. Neurosci. 28, 8161-8168. https://doi.org/10.1523/JNEUROSCI.1093-08.2008

Pooresmaeili, A., FitzGerald, T.H.B., Bach, D.R., Toelch, U., Ostendorf, F., Dolan, R.J., 2014. Cross-modal effects of value on perceptual acuity and stimulus encoding. Proc. Natl. Acad. Sci. U. S. A. 111, 15244-15249. https://doi.org/10.1073/pnas.1408873111

Posner, M.I., 1980. Orienting of Attention. Q. J. Exp. Psychol. 32, 3-25. https://doi.org/10.1080/00335558008248231

Rangel, A., Camerer, C., Montague, P.R., 2008. A framework for studying the neurobiology of valuebased decision making. Nat. Rev. Neurosci. https://doi.org/10.1038/nrn2357

Reynolds, J.H., Chelazzi, L., 2004. Attentional modulation of visual processing. Annu. Rev. Neurosci. https://doi.org/10.1146/annurev.neuro.26.041002.131039

Reynolds, J.H., Chelazzi, L., Desimone, R., 1999. Competitive mechanisms subserve attention in macaque areas V2 and V4. J. Neurosci. 19, 1736-1753. https://doi.org/10.1523/jneurosci.1905-01736.1999

Rock, I., Gutman, D., 1981. The effect of inattention on form perception. J. Exp. Psychol. Hum. Percept. Perform. 7, 275-285. https://doi.org/10.1037/0096-1523.7.2.275

Rossi, V., Vanlessen, N., Bayer, M., Grass, A., Pourtois, G., Schacht, A., 2017. Motivational salience modulates early visual cortex responses across task sets. J. Cogn. Neurosci. 29, 968-979. https://doi.org/10.1162/jocn_a_01093

Rutherford, H.J.V., O'Brien, J.L., Raymond, J.E., 2010. Value associations of irrelevant stimuli modify rapid visual orienting. Psychon. Bull. Rev. 17, 536-542. https://doi.org/10.3758/PBR.17.4.536

Rutkowski, R.G., Weinberger, N.M., 2005. Encoding of learned importance of sound by magnitude of representational area in primary auditory cortex. Proc. Natl. Acad. Sci. U. S. A. 102, 13664 13669. https://doi.org/10.1073/pnas.0506838102

Sanz, L.R.D., Vuilleumier, P., Bourgeois, A., 2018. Cross-modal integration during value-driven $\begin{array}{llll}\text { attentional capture. } & \text { Neuropsychologia } & 120, & 105-112 .\end{array}$ https://doi.org/10.1016/j.neuropsychologia.2018.10.014

Schultz, W., 2015. Neuronal reward and decision signals: From theories to data. Physiol. Rev. 95, 853-951. https://doi.org/10.1152/physrev.00023.2014

Schultz, W., 2013. Updating dopamine reward signals. Curr. Opin. Neurobiol. 23, 229-238. https://doi.org/10.1016/J.CONB.2012.11.012

Schultz, W., 2006. Behavioral Theories and the Neurophysiology of Reward. Annu. Rev. Psychol. 57, 87-115. https://doi.org/10.1146/annurev.psych.56.091103.070229

Schultz, W., 2002. Getting Formal with Dopamine and Reward. Neuron 36, 241-263. https://doi.org/10.1016/S0896-6273(02)00967-4

Schultz, W., 2000. Multiple reward signals in the brain 1, 199-207. https://doi.org/10.1038/35044563

Schultz, W., 1998. Predictive Reward Signal of Dopamine Neurons. J. Neurophysiol. 80, 1-27. 
https://doi.org/10.1152/jn.1998.80.1.1

Schultz, W., 1986. Responses of midbrain dopamine neurons to behavioral trigger stimuli in the monkey. J. Neurophysiol. 56, 1439-1461. https://doi.org/10.1152/jn.1986.56.5.1439

Schultz, W., Dayan, P., Montague, P.R., 1997. A Neural Substrate of Prediction and Reward. Science (80-. ). 275, 1593-1599. https://doi.org/10.1126/science.275.5306.1593

Seitz, A.R., Kim, R., Shams, L., 2006. Sound Facilitates Visual Learning. Curr. Biol. 16, 1422-1427. https://doi.org/10.1016/j.cub.2006.05.048

Serences, J.T., 2008. Value-Based Modulations in Human Visual Cortex. Neuron 60, 1169-1181. https://doi.org/10.1016/j.neuron.2008.10.051

Serences, J.T., Saproo, S., 2010. Population response profiles in early visual cortex are biased in favor of more valuable stimuli. J. Neurophysiol. 104, 76-87. https://doi.org/10.1152/jn.01090.2009

Serences, J.T., Saproo, S., Scolari, M., Ho, T., Muftuler, L.T., 2009. Estimating the influence of attention on population codes in human visual cortex using voxel-based tuning functions. Neuroimage 44, 223-231. https://doi.org/10.1016/J.NEUROIMAGE.2008.07.043

Shadmehr, R., Xivry, J.J.O. de, Xu-Wilson, M., Shih, T.-Y., 2010. Temporal Discounting of Reward and the Cost of Time in Motor Control. J. Neurosci. 30, 10507-10516. https://doi.org/10.1523/JNEUROSCI.1343-10.2010

Shams, L., Seitz, A.R., 2008. Benefits of multisensory learning. Trends Cogn. Sci. 12, 411-417. https://doi.org/10.1016/j.tics.2008.07.006

Shuler, M.G., Bear, M.F., 2006. Reward timing in the primary visual cortex. Science (80-. ). 311, 1606-1609. https://doi.org/10.1126/science.1123513

Sobal, J., 1998. Cultural Comparison Research Designs in Food, Eating, and Nutrition. Food Qual. Prefer. 9, 385-392. https://doi.org/10.1016/S0950-3293(98)00029-9

Stanisor, L., Van Der Togt, C., Pennartz, C.M.A., Roelfsema, P.R., 2013. A unified selection signal for attention and reward in primary visual cortex. Proc. Natl. Acad. Sci. U. S. A. 110, 91369141. https://doi.org/10.1073/pnas.1300117110

Stein, B.E., Meredith, M.A., 1993. The merging of the senses. The MIT Press.

Steinberg, E.E., Keiflin, R., Boivin, J.R., Witten, I.B., Deisseroth, K., Janak, P.H., 2013. A causal link between prediction errors, dopamine neurons and learning. Nat. Neurosci. 16, 966-973. https://doi.org/10.1038/nn.3413

Stine, G.M., Zylberberg, A., Ditterich, J., Shadlen, M.N., 2020. Differentiating between integration and non-integration strategies in perceptual decision making. Elife 9. https://doi.org/10.7554/eLife.55365

Sutton, R.S., Barto, A.G., 2018. Reinforcement Learning: An Introduction. MIT Press, Cambridge; MA.

Takakuwa, N., Kato, R., Redgrave, P., Isa, T., 2017. Emergence of visually-evoked reward expectation signals in dopamine neurons via the superior colliculus in V1 lesioned monkeys. Elife 6. https://doi.org/10.7554/eLife.24459

Takikawa, Y., Kawagoe, R., Itoh, H., Nakahara, H., Hikosaka, O., 2002. Modulation of saccadic eye movements by predicted reward outcome. Exp. Brain Res. 142. https://doi.org/10.1007/s00221001-0928-1

Talsma, D., Woldorff, M.G., 2005. Selective attention and multisensory integration: Multiple phases of effects on the evoked brain activity. J. Cogn. Neurosci. 17, 1098-1114. https://doi.org/10.1162/0898929054475172

Tankelevitch, L., Spaak, E., Rushworth, M.F.S., Stokes, M.G., 2020. Previously Reward-Associated Stimuli Capture Spatial Attention in the Absence of Changes in the Corresponding Sensory Representations as Measured with MEG. J. Neurosci. 40, 5033-5050. https://doi.org/10.1523/JNEUROSCI.1172-19.2020

Theeuwes, J., 2019. Goal-driven, stimulus-driven, and history-driven selection. Curr. Opin. Psychol. https://doi.org/10.1016/j.copsyc.2018.12.024

Theeuwes, J., 2018. Visual selection: Usually fast and automatic; Seldom slow and volitional. J. Cogn. 1. https://doi.org/10.5334/joc.13

Theeuwes, J., Belopolsky, A. V., 2012. Reward grabs the eye: Oculomotor capture by rewarding stimuli. Vision Res. 74, 80-85. https://doi.org/10.1016/j.visres.2012.07.024

Thorndike, E.L., 1911. Animal intelligence. Macmillan, New York. 
Trappenberg, T.P., Dorris, M.C., Munoz, D.P., Klein, R.M., 2001. A model of saccade initiation based on the competitive integration of exogenous and endogenous signals in the superior colliculus. J. Cogn. Neurosci. 13, 256-271. https://doi.org/10.1162/089892901564306

Van Zoest, W., Donk, M., Theeuwes, J., 2004. The role of stimulus-driven and goal-driven control in saccadic visual selection. J. Exp. Psychol. Hum. Percept. Perform. 30, 746-759. https://doi.org/10.1037/0096-1523.30.4.749

Vitay, J., Hamker, F.H., 2007. On the role of dopamine in cognitive vision, in: Lecture Notes in Computer Science (Including Subseries Lecture Notes in Artificial Intelligence and Lecture Notes in Bioinformatics). Springer Verlag, pp. 352-366. https://doi.org/10.1007/978-3-54077343-6_23

Von Kriegstein, K., Giraud, A.L., 2006. Implicit multisensory associations influence voice recognition. PLoS Biol. 4, 1809-1820. https://doi.org/10.1371/journal.pbio.0040326

Wang, L., Yu, H., Zhou, X., 2013. Interaction between value and perceptual salience in value-driven attentional capture. J. Vis. 13, 5-5. https://doi.org/10.1167/13.3.5

Watson, P., Pearson, D., Most, S.B., Theeuwes, J., Wiers, R.W., Le Pelley, M.E., 2019. Attentional capture by Pavlovian reward-signalling distractors in visual search persists when rewards are removed. PLoS One 14, e0226284. https://doi.org/10.1371/journal.pone.0226284

Weil, R.S., Furl, N., Ruff, C.C., Symmonds, M., Flandin, G., Dolan, R.J., Driver, J., Rees, G., 2010. Rewarding Feedback After Correct Visual Discriminations Has Both General and Specific Influences on Visual Cortex. J. Neurophysiol. 104, 1746-1757. https://doi.org/10.1152/jn.00870.2009

Westbrook, A., Frank, M.J., Cools, R., 2021. A mosaic of cost-benefit control over cortico-striatal circuitry. Trends Cogn. Sci. https://doi.org/10.1016/j.tics.2021.04.007

Wild, B., Treue, S., 2021. Primate extrastriate cortical area MST: a gateway between sensation and cognition. J. Neurophysiol. 125, 1851-1882. https://doi.org/10.1152/jn.00384.2020

Wise, R.A., 2004. Dopamine, learning and motivation. Nat. Rev. Neurosci. https://doi.org/10.1038/nrn1406

Wittmann, M., Leland, D.S., Paulus, M.P., 2007. Time and decision making: differential contribution of the posterior insular cortex and the striatum during a delay discounting task. Exp. Brain Res. 20061794 179, 643-653. https://doi.org/10.1007/S00221-006-0822-Y

Wolfe, J.M., 2021. Guided Search 6.0: An updated model of visual search. Psychon. Bull. Rev. 28, 1060-1092. https://doi.org/10.3758/s13423-020-01859-9

Woods, T.M., Recanzone, G.H., 2004. Cross-Modal Interactions Evidenced, in: The Handbook of Multisensory Processes. MIT Press, Cambridge, MA, pp. 35-48.

Yantis, S., Anderson, B.A., Wampler, E.K., Laurent, P.A., 2012. Reward and attentional control in visual search. Nebraska Symp. Motiv. https://doi.org/10.1007/978-1-4614-4794-8-5 


\section{Chapter 2 Differential effects of intra- modal and cross-modal reward value on visual perception: ERP evidence}

Roman Vakhrushev ${ }^{1}$, Felicia Cheng ${ }^{1}$, Annekathrin Schacht ${ }^{2}$, Arezoo Pooresmaeili $^{1 *}$

${ }^{1}$ Perception and Cognition Lab, European Neuroscience Institute Goettingen- A Joint

Initiative of the University Medical Center Goettingen and the Max-Planck-Society,

Germany, Grisebachstrasse 5, 37077 Goettingen, Germany

${ }^{2}$ Affective Neuroscience and Psychophysiology Laboratory, Georg-Elias-Müller-Institute

of Psychology, Georg-August University, Goettingen, Germany

*Corresponding author: a.pooresmaeili@eni-g.de 


\begin{abstract}
Stimuli associated with high reward modulate perception. Such value-driven effects have been shown to originate from the modulation of the earliest stages of sensory processing in the brain. In natural environments objects comprise multiple features (imagine a rolling soccer ball, with its black and white patches and the swishing sound made during its motion), where each feature may signal different associations with previously encountered rewards. How perception of such an object is affected by the value associations of its constituent parts is unknown. The present study compares intra- and cross-modal value-driven effects on behavioral and electrophysiological correlates of visual perception. Human participants first learned the reward associations of visual and auditory cues. Subsequently, they performed a visual orientation discrimination task in the presence of previously rewarded visual or auditory cues (intra- and cross-modal cues, respectively) that were concurrently presented with the target stimulus. During the conditioning phase, when reward associations were learned and reward cues were the target of the task, reward value of both modalities enhanced the electrophysiological correlates of sensory processing in visual cortex. During the postconditioning phase, when reward delivery was halted and previously rewarded stimuli were task-irrelevant, cross-modal value-enhanced behavioral measures of visual sensitivity whereas intra-modal value led to a trend for suppression. A similar pattern of modulations was found in the simultaneously recorded event-related potentials (ERPs) of posterior electrodes. We found an early (90-120 ms) suppression of ERPs evoked by high-value, intramodal stimuli. Cross-modal cues led to a later value-driven modulation, with an enhancement of response positivity for high-compared to low-value stimuli starting at the $\mathrm{N} 1$ window (180-250 ms) and extending to the P3 (300-600 ms) responses of the posterior electrodes. These results indicate that visual cortex is modulated by the reward value of visual as well as auditory cues. Previously rewarded, task-irrelevant cues from the same or different sensory modality have a different effect on visual perception, as intra-modal highvalue cues may interfere with the target processing, whereas cross-modal high-value cues boost the perception of the target.
\end{abstract}




\subsection{Introduction}

Reward seeking is a fundamental mechanism for survival and one of the main predictors of behavior (Berridge and Kringelbach, 2008; Schultz, 2015). We tend to prioritize what we eat, where we go, and what we do based on the expected value of objects or actions learned through experience. A large body of literature has identified a network encompassing the ventral striatum and orbitofrontal cortex to play a key role in learning the associated value of neutral stimuli through experience (Rangel et al., 2008; Schultz, 2000). As natural environments are rich and dynamic, reward information could be conveyed through multiple sensory modalities (e.g., hearing the sound of an approaching ice-cream truck or seeing its characteristic red color both inform us of the possibility of enjoying an ice cream) and reward associations or the goals of the task at hand may change over time (e.g., a red truck can carry other items rather than ice-cream). These features entail a tight interaction between the reward network and the early sensory areas so that stimuli leading to better outcomes such as higher rewards or realization of the goals of the task are prioritized for perceptual processing (Haber, 2011; Pessoa and Engelmann, 2010). In fact, previous research has identified value-driven modulations of neuronal responses in almost all primary sensory areas (Goltstein et al., 2013; Pleger et al., 2008; Rutkowski and Weinberger, 2005; Shuler and Bear, 2006; Stanisor et al., 2013; Weil et al., 2010). In line with the effect of reward on the earliest stages of sensory processing, studies on humans with the use of electroencephalography (EEG) reported reward-related modulations of the visual EventRelated Potentials (ERPs) that are likely to originate from the primary and extrastriate visual cortices (Tankelevitch et al., 2020), including modulations in P1 (80-120 ms) (Bayer et al., 2019; Hammerschmidt et al., 2018; Schacht et al., 2012), N1 (140-220 ms) (Luque et al., 2017) and C1 ( 70 ms) (Bayer et al., 2017; Rossi et al., 2017) ERP components. Despite the robust evidence for an influence of reward on early sensory mechanisms, especially in the visual cortex, it has remained unclear how reward information and sensory processing are coordinated across multiple sensory modalities. This is an important question as in natural environments stimuli are typically multisensory and reward information should be hence coordinated across multiple sensory modalities.

Two recent studies tried to bridge this gap and examined cross-modal reward mechanisms, where an auditory stimulus associated with positive reward value affects visual perception (Leo and Noppeney, 2014; Pooresmaeili et al., 2014). During a conditioning phase, participants associated different pure tones with reward values. In a subsequent postconditioning phase, participants either reported the location (Leo and Noppeney, 2014) or 
the orientation (Pooresmaeili et al., 2014) of a near-threshold Gabor stimulus in the presence of task-irrelevant auditory tones. Importantly, during the post-conditioning phase, auditory tones did not predict the delivery of reward anymore. The first study (Leo and Noppeney, 2014) showed that participants' accuracy in determining the location of the near-threshold Gabor was higher in the presence of tones previously associated with a positive reward compared to no reward (neutral). The authors concluded that the rewarded tones enhanced the bottom-up salience of the visual stimuli hence leading to a better detection and more accurate localization of the near-threshold Gabors. Similar results were found in the second study (Pooresmaeili et al., 2014), as it was shown that participants' orientation discrimination improved in the presence of auditory tones previously associated with high compared to low reward value. With the aid of the simultaneously acquired functional MRI data, it was further shown that the enhanced orientation discrimination is also reflected in the activation patterns of the early visual areas elicited by a specific Gabor orientation (Pooresmaeili et al., 2014). Moreover, in addition to classical reward coding areas (such as Striatum and Orbitofrontal cortex), multisensory regions of the temporal cortex were also modulated by sound values suggesting that they may serve as an intermediary stage to better coordinate the interaction between the primary sensory cortices when high-value stimuli were presented (Pooresmaeili et al., 2014). Subsequent studies (Anderson, 2016a; Kang et al., 2018, 2017; Sanz et al., 2018) used a variety of tasks encompassing visual search or detection or discrimination tasks and confirmed that cross-modal (auditory) reward cues could affect visual perception, albeit the cross-modal reward cues in some cases interfered with the visual task (Anderson, 2016a; Sanz et al., 2018) and in other cases improved it (Kang et al., 2018, 2017). Together, these results provide evidence that reward signals can be broadcasted cross-modally. However, it remains unclear which mechanisms underlie the transmission of reward signals between sensory modalities and their effect on visual perception.

A predominant view posits that reward effects on sensory perception occur through the engagement of attentional mechanisms (Chelazzi et al., 2013; Failing and Theeuwes, 2018; Pessoa, 2015). In this view, rewarding cues receive higher attentional prioritization either through an involuntary, value-driven attentional capture (Anderson et al., 2011a), through voluntary, goal-directed attentional selection (Kiss et al., 2009; Sawaki et al., 2015; Van Den Berg et al., 2014), or through cognitive control mechanisms (Etzel et al., 2016; Pessoa, 2009). In line with this, it has been shown that a reward cue that is aligned with the goal-directed attention in space and in time improves visual performance (Kiss et al., 2009; 
Kristjánsson et al., 2010), whereas when the rewarded stimulus appears away from the target, it interferes with the target detection or discrimination (Anderson et al., 2013; Della Libera and Chelazzi, 2009). Considering these findings, enhancement of visual perception by cooccurring sounds (Leo and Noppeney, 2014; Pooresmaeili et al., 2014) is unexpected, as auditory tones in these studies were irrelevant to the visual task and could potentially act as a high-reward distractor capturing attention away from the visual target. We consider three possible explanations for the observation that high-reward auditory cues could in some cases enhance visual perception. Firstly, cross-modal auditory reward cues may involve a different type of attentional selection compared to the attentional mechanisms involved in the processing of visual targets (Alais et al., 2006). According to this view, although auditory reward cues capture attention, they do not interfere with the task-relevant visual attention as they are processed through a different attentional mechanism. However, this explanation cannot yet explain why rewarded sounds should enhance visual performance. A second possibility is that cross-modal reward cues enhance visual perception by strengthening the audiovisual integration of the auditory and visual components of an audiovisual stimulus. Although audiovisual integration largely occurs automatically (Calvert and Thesen, 2004), top-down factors such as attention (Alsius et al., 2005; Navarra et al., 2010; Talsma et al., 2007) and recently reward value (Bean et al., 2021; Cheng et al., 2020) have been shown to affect its strength. Through a more efficient integration with the visual cues, auditory reward signals could hence capture attention not only to themselves but also to the whole audiovisual object, including the visual target, thereby improving performance. In fact, the boost of integration may be a key characteristic of reward modulation where the association of one sensory property of an object with higher reward spreads to all sensory properties of the same object hence promoting their grouping, as has been shown before (Stanisor et al., 2013). Lastly, cross-modal and intra-modal reward cues could influence visual perception at different stages of sensory processing (Anderson et al., 2011a; Yantis et al., 2012). While intra-modal, task-irrelevant reward cues may interfere with the early bottom-up processing of the visual target, cross-modal reward cues could enhance the later sensory or post-sensory decision stages (Franzen et al., 2020). These possible scenarios can be disentangled by comparing cross-modal reward effects against intra-modal reward effects, i.e., when the reward cue and target cues appear in the same sensory modality. This would also determine whether common or modality-specific mechanisms underlie reward-driven modulation of sensory perception. However, previous studies have not undertaken such a comparison. 
The central aim of this study was to provide a better understanding of reward effects across sensory modalities by comparing intra- and cross-modal reward effects while using the high temporal resolution of the EEG data to delineate different stages of stimulus processing across time. To this end, we employed a task design similar to a previous study (Pooresmaeili et al., 2014), where participants first learned the reward value of visual or auditory cues during a conditioning phase and subsequently performed a visual orientation task in the presence of previously rewarded cues. During the latter post-conditioning phase, auditory and visual reward cues were presented simultaneously with the target stimulus and at the same side of the visual field. Crucially reward cues during the post-conditioning phase were irrelevant to the task at hand and did not predict the delivery of reward (i.e. no-reward phase). Measuring reward effects at a no-reward phase has proven to be an effective method to separate different modulations of perception, i.e., those related to the long-term associative value of reward cues from those of goal-directed boosts driven by rewardpredicting cues or the delivery of the reward itself (Anderson and Yantis, 2013; Failing and Theeuwes, 2015; Maclean and Giesbrecht, 2015; Mine and Saiki, 2015; Rutherford et al., 2010; Watson et al., 2019). Association of task-irrelevant cues, but not the target, with reward values allows dissociating reward effects and effects of selective attention involved in the processing of the target stimulus. Thus, the current study will use task-irrelevant intraand cross-modal reward cues during a no-reward phase (here referred to as postconditioning) to measure reward effects across sensory modalities on visual perception.

We hypothesized that cues previously associated with high value should positively influence behavioral performance (i.e., enhancing the target discriminability and reducing the reaction times) and early posterior ERP components (i.e., increasing the amplitudes of P1 and N1 components, and decreasing their latencies). This hypothesis is based on a mechanism where higher reward value not only prioritizes the processing of rewardassociated cues but also promotes the integration of different components of an object into a coherent perceptual group. With this, we expected that the reward-related enhancements should spread to other sensory components of the same object, irrespective of the sensory modality of the reward cue. Next, we hypothesized that reward-related modulations of P1 and N1 components in posterior electrodes occur earlier and are stronger when the reward cue is delivered intra-modally (in visual modality) compared to when the reward cue is delivered cross-modally (in auditory modality), as intra-modal effects rely on direct neuronal connections between reward and target sensory representations (Serences, 2008; Serences and Saproo, 2010), whereas cross-modal effects rely on the long-range communication 
between different brain areas and/or involve intermediate stages (Pooresmaeili et al., 2014). Finally, we hypothesized that in later stages of information processing (i.e.,> $250 \mathrm{~ms}$ ), intraand cross-modal reward cues elicit similar modulations of ERP amplitudes, both leading to an enhancement of P3 ERP component.

\subsection{Material and Methods}

\section{Participants}

Thirty-eight participants took part in our experiment (23 women, mean age \pm SD: 25.8 \pm 5 ). Two participants were excluded since their performance during the associative reward learning task indicated that they either did not learn the reward associations $(\mathrm{N}=1)$ or had difficulties with discriminating the location of the stimuli $(\mathrm{N}=1)$. The final sample consisted of 36 healthy participants with normal or corrected-to-normal vision who had no history of neurophysiological or psychiatric disorders, according to a self-report. Participants gave written informed consent after the experimental procedures were clearly explained to them. The study was conducted in full accordance with the Declaration of Helsinki and was approved by the local Ethics Committee of the Medical University Göttingen (proposal: 15/7/15).

The sample size, all procedures, and the analysis plan of the study were preregistered (https://osf.io/47wxr/). In brief, the required sample size for this study was calculated based on the effect size observed in a pilot study $(\mathrm{N}=8)$. To this end, we averaged the reward effect on the amplitude of the P1 component of the posterior ERPs across cross- and intra-modal cues during post-conditioning. The sample size was then calculated for a within-subjects, paired, two-tailed t-test for a significant difference between high and low-value stimuli with alpha $=0.05$ and power $(1-$ Beta $)=0.8$. in GPower software (Faul et al., 2007). This power analysis indicated that at least 33 subjects were needed (mean \pm SD of effect size: $0.35 \pm$ 0.69 for the difference between high and low value, $\left.d_{z}=0.5097\right)$. Based on this and to maintain the counterbalancing of our experimental conditions ( 4 possible combinations of auditory and visual cues with high or low reward), we aimed for an a priori sample size of $\mathrm{N}=36$ before the data collection started.

\section{Experimental design}

\section{Study structure}

Each participant completed three phases of the experiment: a pre-conditioning phase (Figure 1b), an associative reward learning (conditioning) phase (Figure 1a), and finally, a 
post-conditioning phase (Figure 1b): During the pre-conditioning phase, participants performed a visual orientation discrimination task at their discrimination threshold (see Stimuli and equipment) in the presence of the task-irrelevant auditory or visual cues. In the conditioning phase, participants learned reward values of the visual and auditory cues with a cue localization task. Finally, the post-conditioning phase employed the same task as the pre-conditioning, but the auditory or visual cues had now been associated with a specific reward value during conditioning. We used the pre-conditioning phase to control for perceptual biases towards visual and auditory cues that were independent of their reward value. Therefore, all effect sizes (Figure 3-6) are reported after the subtraction of the preconditioning data from the corresponding data during post-conditioning.

\subsubsection{Conditioning phase}

During the conditioning phase (Figure 1a), participants reported the location of a peripheral visual $\left(9^{\circ}\right.$ eccentricity) or auditory stimulus (sound played in the left or right earphone) by pressing left or right arrow keyboard buttons. Correct responses were followed by a monetary reward, the magnitude of which depended on the respective color or pitch of the visual or auditory cues. Reward information was presented at the screen center both in written format (with digits showing the number of Euro cents obtained) and graphically (a color bar illustrating the ratio of the reward magnitude in the current trial to the maximum possible level in the experiment, respectively), and stayed in view for $1.5 \mathrm{~s}$. Rewards were drawn from two Poisson distributions with the mean of 25 cents and SD of 4.8 cents for high-reward trials and a mean of 2 cents and SD 1.4 cents for low-reward trials (minimum and maximum reward was fixed at 35 and 0.4 cents, respectively). Participants were instructed to remember and report the color and the sound pitch that delivered higher rewards at the end of the conditioning phase and at the end of the experiment. The location (left or right), modality (auditory or visual), and identity (color or sound pitch) of the cues were pseudorandomized across trials with the constraint that the same condition could not be repeated more than twice in a row. The association of each cue identity with reward value was counterbalanced across participants. 

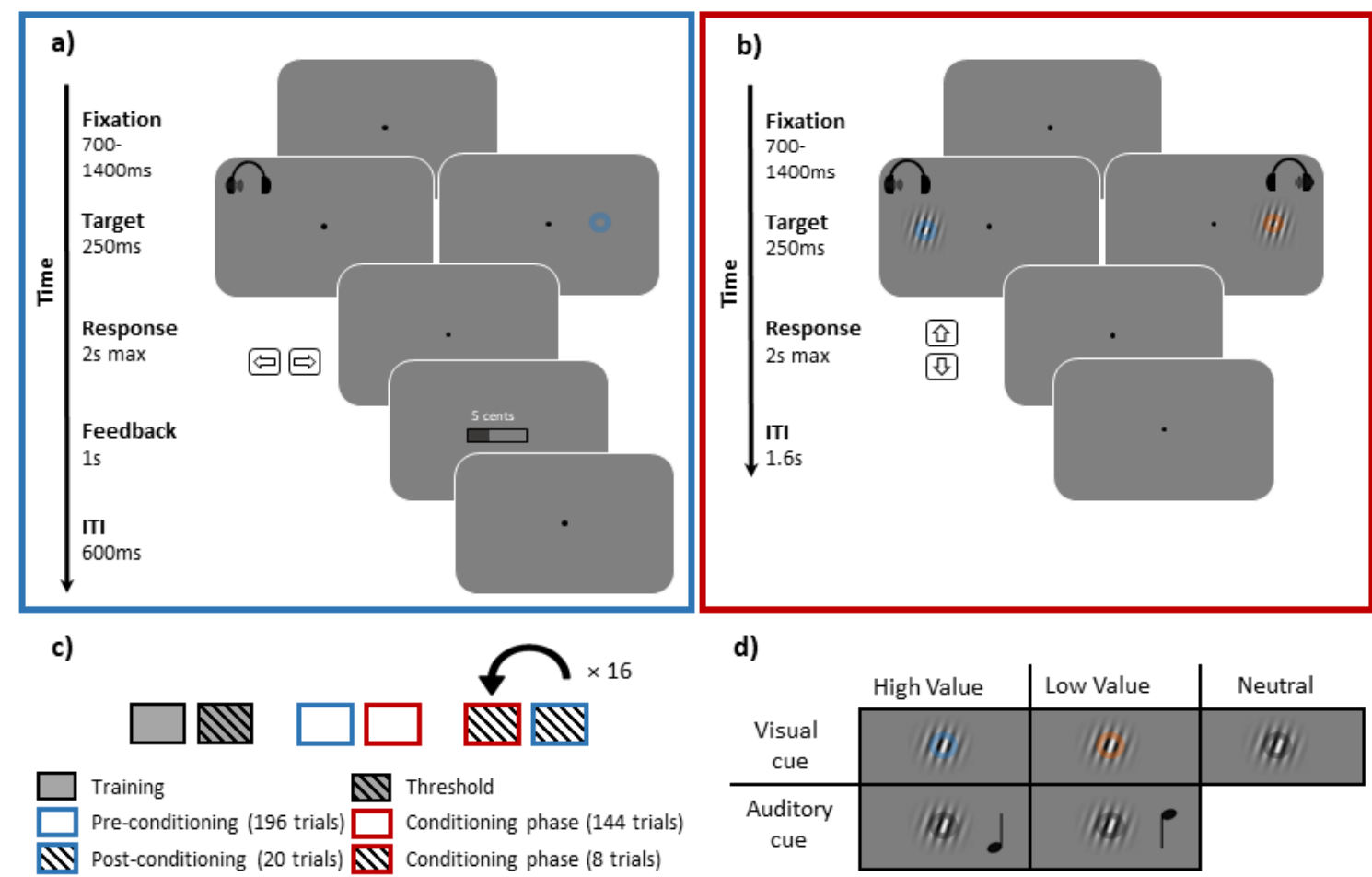

Figure 1. Stimuli and experimental procedure. a) Reward learning phase (conditioning). Participants learned the reward association of the visual or auditory cues by performing a localization task and observing their monetary rewards contingent on the color (visual) or pitch (auditory) of the cues. In this task, after an initial fixation period (700-1400 ms), a visual or an auditory cue was presented to the left or right side of the fixation point and participants had to localize them by pressing either left or right arrow buttons of a keyboard (maximum response time $2 \mathrm{~s}$ ). Here, stimuli in two example trials are shown, one with an auditory cue presented to the left and the other with a visual cue presented to the right. The correct performance led to either a high or a low monetary reward dependent on the identity of the cue (color of visual cues and pitch of auditory cues). b) Orientation discrimination task employed during the pre- and post-conditioning phases. To probe the effects of reward value on visual sensitivity, an orientation discrimination task was employed. A trial of this task started with a fixation period $(700-1400 \mathrm{~ms})$, followed by the presentation of a peripheral Gabor stimulus $\left(9^{\circ}\right.$ eccentricity). Participants were instructed to discriminate the orientation of the Gabor stimulus (clockwise or counterclockwise tilt) by pressing up or down arrow buttons on a keyboard (maximum response duration $=2 \mathrm{~s}$ ). Concurrent with the Gabor, a visual or auditory cue was also presented (intra- or cross-modal cues, respectively). Intra- and cross-modal cues were irrelevant to the orientation discrimination task and did not predict reward delivery. c) The schematic illustration of the different stages of an experimental session. d) Design matrix of all stimulus conditions used during the test phase.

Participants completed 280 trials with the cue localization task to learn reward associations. From these, 144 trials were presented during an initial longer conditioning block (comprising 36 repetitions of value $\times$ modality conditions). Subsequently, 136 trials of the conditioning phase were divided into 16 short blocks ( 8 trials per block) and then interleaved with short blocks of the post-conditioning phase (20 trials per block) to prevent the extinction of the reward associations (Figure 1c, also see under Pre- and postconditioning phases).

\section{Pre- and Post-conditioning phases}

During pre- and post-conditioning phases, participants performed an orientation discrimination task (Figure 1b). Each trial started with a fixation period (700-1400 ms) 
followed by a Gabor stimulus. The Gabor stimulus was displayed at $9^{\circ}$ eccentricity on the left or right side from the fixation point for $250 \mathrm{~ms}$ (see also Stimuli and equipment). Simultaneously with the Gabor stimulus, an ipsilateral visual (intra-modal) or auditory (cross-modal) cue appeared. Participants were instructed to focus on judging the orientation of the Gabor and ignore irrelevant visual and auditory cues. During these phases, correct responses in the orientation discrimination task granted them a fixed reward shown at the end of the experiment. These instructions aimed to emphasize that previously rewarded cues were not relevant to the task and did not lead to differential immediate rewards anymore.

In total, participants performed 160 trials in the pre-conditioning phase and 320 trials in the post-conditioning phase, with 32 and 64 repetitions of each of the value (high, low, neutral $\times$ modality (intra-modal, cross-modal). In the neutral condition, the Gabor was presented without an auditory tone or color and served as a control condition to characterize the behavioral performance and evoked potentials, irrespective of the reward value (Figure 1c-d).

\section{Stimuli and equipment}

Visual reward cues consisted of a transparent ring overlaid on the Gabor patch $\left(0.44^{\circ}\right.$ in diameter, 0.17 pixels thick, alpha 50\%, color in $\mathrm{HSV}=[20,0.4, \mathrm{~V}]$ for orange, $[250,0.4, \mathrm{~V}]$ for blue and $[0,0, \mathrm{~V}]$ for grey colors and $\mathrm{V}$ was calibrated for each participant to produce iso-luminance, intra-modal conditions contained either a blue or an orange ring whereas cross-modal and neutral conditions contained a grey ring). Auditory reward cues were pure monaural tones, lateralized to the left or right earphone (pitch $=350 \mathrm{~Hz}$ or $1050 \mathrm{~Hz}$ and sound level pressure $=70 \mathrm{~dB}$, adjusted with a digital sound-level meter; SL-100, Voltcraft, Hirschau; separately for each tone and delivered through in-ear earphones ER-3C, Etymotic Research Inc.). Auditory cues were not localized in space as we aimed to keep their characteristics identical to a previous study (Pooresmaeili et al., 2014). Gabor stimuli were Gaussian-windowed sinusoidal gratings with an SD of $0.33^{\circ}$, a spatial frequency of 3 cycles per degree, $2^{\circ}$ diameter, and $50 \%$ contrast, displayed on a gray background of $34.4 \mathrm{~cd} / \mathrm{m}^{2}$ luminance. Before the experiment, participants performed a color adjustment procedure to match the brightness of the visual reward cues. After that, they underwent a short training session to get familiar with the orientation discrimination task (Number of trials $=36$, Figure 1c). After training, participants' orientation discrimination threshold for a performance of $70 \%$ was determined using a QUEST procedure, followed by the main experiment (Figure 1c). Throughout the experiment, trials were repeated in case participants made an eye movement $\left(>2^{\circ}\right.$ displacement of gaze from the fixation dot during fixation or target period), 
used an undesignated button to respond, or did not respond at all to maintain equal number of repetitions across conditions.

Data collection was done in a darkened, sound-attenuated, and electromagnetically shielded chamber. Participants sat $91 \mathrm{~cm}$ away from a 22.5-inch calibrated monitor (ViewPixx/EEG inc., resolution $=1440 \times 980$ pixels, refresh rate $=120 \mathrm{~Hz}$ ) with their heads resting on a chinrest. Stimulus presentation was controlled in Psychophysics toolbox-3 (Brainard, 1997) in MATLAB (version R2015b) environment. Eye movements were recorded with an Eyelink 1000 eye tracker system (SR Research, Ontario, Canada) in a desktop mount configuration, recording the right eye at a sampling rate of $1000 \mathrm{~Hz}$. Electrophysiological data were recorded from 64 electrodes (BrainVision Recorder 1.23.0001 Brain Products GmbH, Gilching, Germany; actiCap, Brain Products GmbH, Gilching, Germany), online referenced to TP9, digitized at $1000 \mathrm{~Hz}$, and amplified with a gain of 10,000. Electrode impedances were kept below $10 \mathrm{k} \Omega$.

\section{Data Analysis}

\section{Analysis of the behavioral data}

For behavioral assessment of visual sensitivity during the orientation discrimination task, we used participants' d-prime scores (d'). D-prime was measured based on the probability of hits and false-alarms, as $\mathrm{d}^{\prime}=\mathrm{Z}\left(\mathrm{P}_{\mathrm{Hit}}\right)-\mathrm{Z}\left(\mathrm{P}_{\mathrm{FA}}\right)$, where one of the tilt directions was arbitrarily treated as "target-present" as in formal Signal Detection Theory analysis of discrimination tasks (Macmillan and Creelman, 1991; Leo et al., 2011). Extreme values of $\mathrm{P}_{\text {Hit }}$ or $\mathrm{P}_{\mathrm{FA}}$ were slightly up- or down-adjusted (i.e., a probability equal to 0 or 1 was adjusted by adding or subtracting 0.001 , respectively). Reaction times in all phases of the experiment were calculated as the elapsed time between the onset of the target stimulus and the participant's response. The resulting response times were averaged for each experimental condition (including correct and incorrect responses).

Outliers were removed from the behavioral and ERP data of each participant $(0.38 \% \pm$ 0.6 SD across subjects). A trial was considered to be an outlier if the gaze fixation during the target presentation period was suboptimal (eye position $>0.9^{\circ}$ from the fixation point) or the reaction time was faster than $10 \mathrm{~ms}$.

\section{Analysis of Event-Related Potentials (ERPs)}

The EEG data was imported and processed offline using EEGLAB (Delorme and Makeig, 2004), an open-source toolbox running under the MATLAB environment. First, an automatic bad channel detection and removal algorithm was applied (using EEGLAB's 
pop_rejchan method; threshold $=5$, method $=$ kurtosis). Later, data of each participant was band-pass filtered with $0.1 \mathrm{~Hz}$ as the high-pass cutoff and $40 \mathrm{~Hz}$ as the low-pass cutoff frequencies. After this, the epochs were extracted by using a stimulus-locked window of $1900 \mathrm{~ms}$ (-700 to $1200 \mathrm{~ms}$ ) and subjected to an Independent Component Analysis (ICA) algorithm (Delorme and Makeig, 2004). Blinks and eye-movement artifacts were automatically identified and corrected using an ICA-based automatic method, implemented in the ADJUST plugin of EEGLAB (Mognon et al., 1994). Bad channels were interpolated using the default spherical interpolation method of the EEGLAB toolbox. Next, data were re-referenced to the average reference. To calculate ERPs, epochs were extracted using a window of $900 \mathrm{~ms}(-100$ to $800 \mathrm{~ms})$ time-locked to the onset of the target, and baselinecorrected using the pre-stimulus time interval (-100 to $0 \mathrm{~ms})$.

Our ERP analysis focused mainly on the latencies and amplitudes of the visual P1 and $\mathrm{N} 1$ components of the visual and auditory reward cues averaged across a region of interest (ROI) comprising four posterior electrodes (PO7/PO8, O1/O2). As auditory and visual cues have different response latencies and reward associations may also affect response latencies, the following method was used to tailor P1 and N1 amplitude measures to each specific condition. ERP components were defined individually for each condition as a $30 \mathrm{~ms}$ window around the peak centered on the grand average ERP wave across participants. The peak of P1 component was defined as the most positive peak within 70-170 ms window, and the peak of N1 component was defined as the most negative peak within 180-250 ms window. Latencies of P1 and N1 peaks were measured by finding the corresponding positive or negative peaks of the ERPs within the corresponding time windows for each participant. Finally, modulations of the P3 component were quantified as the average ERP amplitude between 300-600 ms across all electrodes of the midline ROI (Fz, FCz, Cz, CPz, and Pz).

To determine the onset of intra- and cross-modal reward effects, we calculated the difference between high- and low-value ERPs of each cue type within a $10 \mathrm{~ms}$ moving window against their baseline difference (i.e., within $100 \mathrm{~ms}$ before the stimulus onset). Amplitude differences between 50-800 ms after stimulus onset that reached significance (uncorrected $\mathrm{p}<0.05$ ) were considered as significant value-driven modulations. To account for the inflated probability of type I errors that appear from application of multiple comparisons, only results that were significant in two or more consecutive time windows will be reported (Talsma and Woldorff, 2005).

All procedures described above strictly followed our pre-registered analysis plan. However, after data acquisition and visual inspection of the ERPs, we undertook the 
following modifications and exploratory analyses. Firstly, in the post-conditioning phase, we observed ERP modulations in time intervals that preceded our pre-registered time windows. To examine these early effects, we performed an exploratory analysis in a time window between 90-120 ms after the stimulus onset (referred to as the PA component). The timing of this component corresponds to the timing of the earliest positive peak of visual ERPs observed in previous studies (Luck et al., 1994; Mangun, 1995). It should be noted that in our experiments, reward cues and the Gabor stimulus were presented at the same time. As such, whereas the highest positive peak of P1 that has been the basis of our estimates of P1 component is likely to reflect the responses to both reward cues and the Gabor, the earlier PA component may reflect primarily the response to the reward cues. This conclusion is also supported by inspecting the PA responses to the neutral stimulus depicted in Figure 4a and Supplementary Figure 4a, where PA responses start to emerge only after the reward associative learning (in the post-conditioning phase) when participants pay attention to the identity of different cues. The second exploratory analysis was to inspect the stimulusevoked contra-lateral ERPs. As visual areas are known to have a higher sensitivity to the contralateral stimuli (Woldorff et al., 1997), we also tested the responses of the posterior ROI to contralateral stimuli (i.e., responses of O1 and PO7 to the stimuli on the right visual hemifield and $\mathrm{O} 2$ and PO8 to the stimuli on the left visual hemifield) and measured the correlation of contralateral responses with behavior (Figure 6, Supplementary Figure 4), as exploratory analyses. Finally, ERP modulations occurring at a time window overlapping with the P3 responses (300-600 ms) planned to only be analyzed in midline electrodes were also inspected in the posterior $\mathrm{ROI}(\mathrm{O} 1, \mathrm{O} 2, \mathrm{PO} 7, \mathrm{PO} 8)$ during both conditioning and postconditioning phases.

\section{Statistical Analysis}

We used 2 by 2 repeated measures ANOVAs (rm-ANOVAs) to test the effect of Reward Value (high, low) and Modality (visual, auditory) on behavioral (d' and RT) and electrophysiological responses of the conditioning and post-conditioning phases. To remove the effect of perceptual biases that participants may have for different colors or tone pitches prior to the learning of cues' reward associations, we corrected the behavioral and ERP results of each participant by subtracting the data of each condition in pre-conditioning from the data of post-conditioning. The corrected results were then subjected to rmANOVAs. Planned, paired t-tests were used for pairwise comparisons. Effect sizes in rmANOVAs are reported as partial eta-squared $\left(\eta_{p}^{2}\right)$ and in pairwise comparisons as Cohen's d; i.e. $\mathrm{d}_{\mathrm{z}}$ (Lakens, 2013). 


\subsection{Results}

\section{Conditioning phase}

\section{Behavioral results}

Participants' performance in this task was near perfect as accuracies for both cues were $>95 \%$ (99\% $\pm 1 \%$ for auditory and $100 \% \pm 0 \%$ visual cues). Analysis of reaction times (RTs) revealed a significant main effect of modality $\left(\mathrm{F}_{(1,35)}=70.44, p<0.001, \eta_{\mathrm{p}}{ }^{2}=0.67\right)$. Participants localized visual cues (mean \pm s.e.m.: $501 \pm 12 \mathrm{~ms}$ ) faster than auditory cues (mean \pm s.e.m.: $584 \pm 16 \mathrm{~ms}$ ), in line with the superior performance of vision in localization tasks (Bertelson and Radeau, 1981; Pick et al., 1969). We did not observe a main effect of reward value $\left(\mathrm{F}_{(1,35)}<1\right)$; however, an interaction was found between reward value and modality $\left(\mathrm{F}_{(1,35)}=7.68, p=0.009, \eta_{\mathrm{p}}^{2}=0.18\right)$. For auditory cues, high reward value slowed down responses (mean \pm s.e.m.: $589 \pm 17 \mathrm{~ms}$ and $580 \pm 16 \mathrm{~ms}$ for high and low value cues, respectively), while in the visual modality, high-value cues sped up responses (mean \pm s.e.m.: $496 \pm 13 \mathrm{~ms}$ and $506 \pm 12 \mathrm{~ms}$ for high- and low-value cues, respectively). The effect of reward value separately in each modality did not reach significance $(\mathrm{t}(35)=-2.02, p=$ $0.051, \mathrm{~d}_{\mathrm{z}}=0.34$ and $\mathrm{t}(35)=1.52, p=0.137, \mathrm{dz}=0.253$ for visual and auditory cues, respectively).

\section{ERP responses: $P 1, N 1, P 3$}

We next examined whether the posterior ROI (PO7/PO8 and O1/O2, reflecting the activity of occipital and parietal visual areas) that we had planned to use for probing reward effects during the main orientation discrimination task exhibits reliable reward modulations (ERP amplitude and or latency) during reward associative learning (Figure 2). The rationale for examining visual ERPs in the conditioning phase is twofold. Firstly, in this phase reward modulations in the posterior ROI serve as an index of how value information affects sensory processing when reward cues are the target of the task (task-relevant). Secondly, of particular interest are reward modulations evoked by auditory cues, as such modulations would indicate that reward information is broadcasted to visual cortex even in the absence of concomitant visual stimulation.

According to the rmANOVA, $\boldsymbol{P 1}$ amplitudes were impacted by modality $\left(\mathrm{F}_{(1,35)}=\right.$ $12.89, p<0.001, \eta_{\mathrm{p}}{ }^{2}=0.27$ ), with higher amplitudes for auditory (mean \pm s.e.m: $3.66 \pm 0.28$ $\mu \mathrm{V}$ ) than visual cues (mean \pm s.e.m: $2.27 \pm 0.24 \mu \mathrm{V})$. Effects of reward values $\left(\mathrm{F}_{(1,35)}<1, p\right.$ $=0.919)$ and the modality by reward interaction $\left(\mathrm{F}_{(1,35)}<1, p=0.971\right)$ did not reach significance. Analysis of $\boldsymbol{P 1}$ latency revealed a main effect of modality $\left(\mathrm{F}_{(1,35)}=53.1, p<\right.$ 
$0.001, \eta_{\mathrm{p}}{ }^{2}=0.60$ ), reflecting an earlier onset of $\mathrm{P} 1$ responses for auditory (mean \pm s.e.m.: $130.7 \pm 2 \mathrm{~ms}$ ) compared to visual cues (mean \pm s.e.m.: $155.3 \pm 2.7 \mathrm{~ms}$ ). Neither a main effect of reward value $\left(\mathrm{F}_{(1,35)}=1.19, p=0.284\right)$ nor an interaction between reward value and modality $\left(\mathrm{F}_{(1,35)}=2.72, p=0.108\right)$ was found.

Analysis of N1 amplitudes in the conditioning phase revealed a main effect of modality $\left(\mathrm{F}_{(1,35)}=17.42, p<0.001, \eta_{\mathrm{p}}{ }^{2}=0.33\right)$, again with larger amplitudes for auditory (mean \pm s.e.m: $-1.36 \pm 0.35 \mu \mathrm{V}$ ) compared to visual cues (mean \pm s.e.m: $0.73 \pm 0.32 \mu \mathrm{V}$ ). Again, neither the main effect of reward value nor the reward by modality interaction reached significance (all ps $>0.1)$. N1 latency was not modulated by modality $\left(\mathrm{F}_{(1,35)}=1.92\right.$, $p=0.175)$ or by reward value $\left(\mathrm{F}_{(1,35)}<1, p=0.477\right)$. However, a significant interaction was found between reward value and modality $\left(\mathrm{F}_{(1,35)}=7.07, \mathrm{p}=0.012, \eta_{\mathrm{p}}{ }^{2}=0.17\right)$, reflecting different direction of value-driven modulation of $\mathrm{N} 1$ latencies in the two modalities. Whereas visual high value cues significantly sped up the N1 peak (mean \pm s.e.m.: $208.47 \pm$ $2.86 \mathrm{~ms}$ and $215.58 \pm 2.99 \mathrm{~ms}$ and for high and low-value cues respectively, $\mathrm{t}(35)=-2.54, p$ $=0.016, \mathrm{~d}_{\mathrm{z}}=0.424$ ), auditory high-value cues tended to slow down $\mathrm{N} 1$ responses (mean \pm s.e.m.: $218 \pm 3.3 \mathrm{~ms}$ and $214.7 \pm 3.2 \mathrm{~ms}$ for high and low value cues respectively, $\mathrm{t}(35)=$ $1.12, \mathrm{p}=0.27$, Cohen's $\mathrm{d}=0.19$ ).

[Insert Table. 1 here]

\begin{tabular}{lc|c|c|c} 
Amplitude $(\mu \mathrm{V})$ & \multicolumn{2}{c}{$\mathrm{P} 1$} & $\mathrm{~N} 1$ & $\mathrm{P} 3$ \\
& $\mathrm{VH}$ & $2.26 \pm 0.25$ & $0.62 \pm 0.33$ & $2.85 \pm 0.42$ \\
& $\mathrm{VL}$ & $2.27 \pm 0.26$ & $0.84 \pm 0.33$ & $2.74 \pm 0.38$ \\
\cline { 3 - 5 } & $\mathrm{AH}$ & $3.65 \pm 0.29$ & $-1.22 \pm 0.36$ & $2.88 \pm 0.27$ \\
\cline { 3 - 5 } & $\mathrm{AL}$ & $3.66 \pm 0.31$ & $-1.49 \pm 0.39$ & $2.56 \pm 0.29$
\end{tabular}

Latency (ms)

\begin{tabular}{cc|c}
\multicolumn{1}{c|}{ P1 } & N1 \\
\cline { 2 - 3 } VH & $154.67 \pm 3.03$ & $208.47 \pm 2.86$ \\
\cline { 2 - 3 } VL & $155.86 \pm 3.68$ & $215.58 \pm 2.99$ \\
\cline { 2 - 3 } AH & $134.94 \pm 3.30$ & $218.47 \pm 3.23$ \\
\cline { 2 - 3 } AL & $126.56 \pm 2.97$ & $214.69 \pm 3.24$
\end{tabular}

Table 1. Amplitude and latencies of visual ERP components evoked by visual high value (VH), visual low value (VL), auditory high value (AH), and auditory low value (LA) cues during the conditioning phase, measured in the posterior ROI.

Analysis of $\boldsymbol{P 3}$ amplitude in the posterior ROI (PO7, O1, O2, PO8), quantified between 300 and $600 \mathrm{~ms}$, revealed a main effect of reward value $\left(\mathrm{F}_{(1,35)}=4.82, p=0.035\right.$, 
$\eta_{\mathrm{p}}{ }^{2}=0.121$ ), reflecting larger amplitudes for high-reward cues (mean \pm s.e.m.: $2.86 \pm 0.29$ $\mu \mathrm{V}$ ) than for low-reward cues (mean \pm s.e.m.: $2.65 \pm 0.3 \mu \mathrm{V}$ ). The main effect of modality $\left(\mathrm{F}_{(1,35)}<1, p=0.816\right)$ and an interaction effect between modality and value $\left(\mathrm{F}_{(1,35)}<1, p=\right.$ 0.458) were non-significant.

We note that this analysis is exploratory as in our pre-registered plan we intended to only examine the $\mathrm{P} 3$ component in midline electrodes $(\mathrm{Pz}, \mathrm{CPz}, \mathrm{Cz}, \mathrm{FCz}, \mathrm{Fz})$ in a time window of 300-600 ms. However, examination of the topographic distribution of these ERP modulations within this window suggested a rather posterior topography than the expected more centrally distributed P300 (Figure 2a-b). Hence, we decided to also examine P3 amplitudes in a posterior ROI (O1, O2, PO7, PO8). Similar motivational modulation of posterior P3-like responses has been observed in previous studies (Baines et al., 2011). 

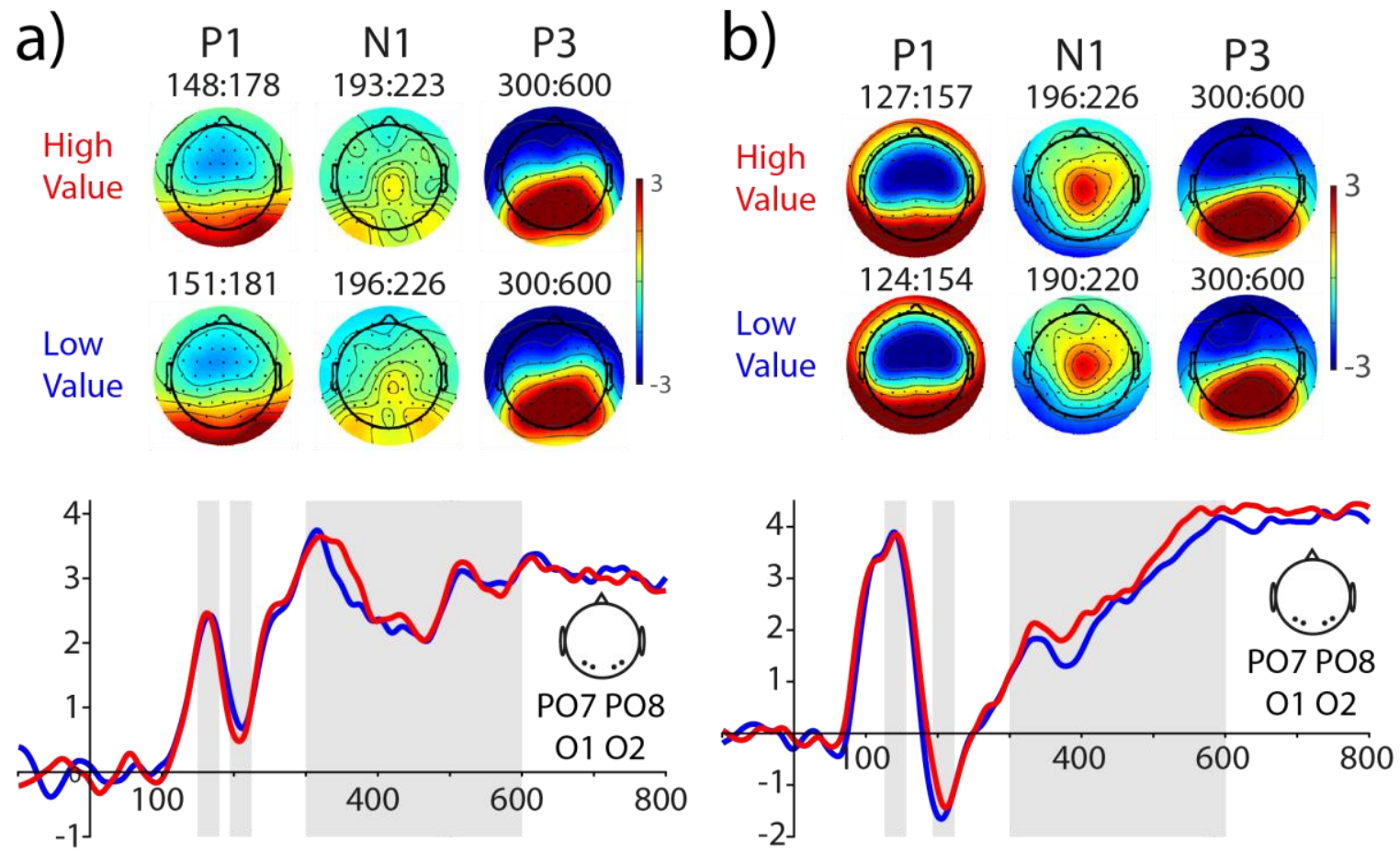

C) P3 Amplitude

d)

N1 Latency
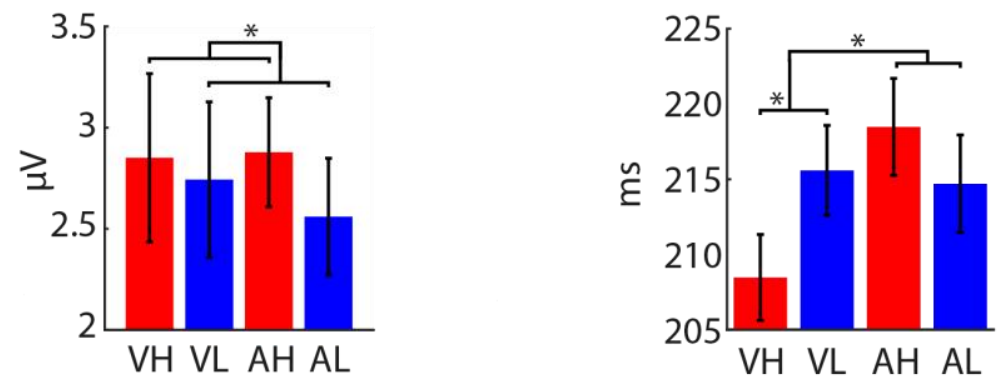

Figure 2. ERP responses of the posterior ROI to the visual and auditory reward cues during the conditioning phase. a) ERPs of visual reward cues with high (red traces) and low (blue traces) values measured in a posterior $\mathrm{ROI}(\mathrm{O} 1, \mathrm{O} 2, \mathrm{PO} 7$, and PO8). The shaded grey areas correspond to $30 \mathrm{~ms}$ windows around the peak of P1 (70-170 ms) and N1 (180-250 ms) used to estimate the amplitude of these components for each condition (here the window is averaged across high and low-value conditions) and the window used to estimate P3 responses (300-600 ms). The topographic distribution of P1, N1 and P3 components are shown for each reward value condition. b) Same as a for auditory reward cues. c-d) analysis of the amplitude and latency of the ERP components revealed significant value-driven modulations for the amplitude of P3 shown in $\mathbf{c}$ and the latency of the N1 component shown in d. Visual High Value: VH; visual Low Value: VL; Auditory High Value: AH; Auditory Low Value: AL.

In summary examination of the posterior ROI $(\mathrm{O} 1, \mathrm{O} 2, \mathrm{PO} 7$, and PO8) revealed a significant interaction of reward value and reward modality on the latency of N1 responses, with a significant speeding up of ERP responses to high-value compared to low-value visual cues, and opposite trend for auditory N1 responses. In the P3 window, a main effect of reward value was found across all high- compared to low-reward value conditions. Taken together, these results indicate that the higher associative value of both visual and auditory cues enhances their sensory processing in visual areas during the associative learning phase of our experiment. 


\section{Post-Conditioning phase}

\section{Behavioral results}

To assess the behavioral visual sensitivity, participants' $\boldsymbol{d}$-prime scores (d' Post minus d' Pre) were subjected to an ANOVA with reward value (high or low) and modality (intraor cross-modal) as independent factors (Table 2 and Figure 3). This analysis revealed no main effect of modality $\left(\mathrm{F}_{(1,35)}<1, p=0.99\right)$ or reward value $\left(\mathrm{F}_{(1,35)}<1, p=0.35\right)$. Importantly, an interaction was found between modality and reward value $\left(\mathrm{F}_{(1,35)}=5.75, p\right.$ $\left.=.022, \eta_{\mathrm{p}}{ }^{2}=0.14\right)$. Whereas cross-modal reward value enhanced visual sensitivity (mean \pm s.e.m: $0.44 \pm 0.19$, for the difference between high and low value stimuli, corrected for preconditioning, $\left.\mathrm{t}(35)=2.31, p=0.027, \mathrm{~d}_{\mathrm{z}}=0.38\right)$, intra-modal reward value led to a decrement in visual sensitivity (mean \pm s.e.m.: $-0.18 \pm 0.19$, for the difference between high and low value stimuli, corrected for pre-conditioning). The performance decrement for highcompared to low-value intra-modal condition however, did not reach statistical significance $\left(t(35)=-0.97, p=0.34, d_{z}=0.162\right)$. Analysis of the reaction times $(R T)$ revealed a trend for an overall slowing down of responses for high- compared to low-value stimuli, but this effect did not reach statistical significance $\left(\mathrm{F}_{(1,35)}=2.36, p=0.133\right)$. Other main and interaction effects were non-significant (all Fs $<1$, all ps $>0.1$ ).

In summary, during the post-conditioning phase, cross-modal high-value cues improved the visual sensitivity of orientation discrimination while intra-modal, high-value cues showed a trend for interference compared to low-value cues. Reaction times were not significantly affected by the reward value of either type. 
a)

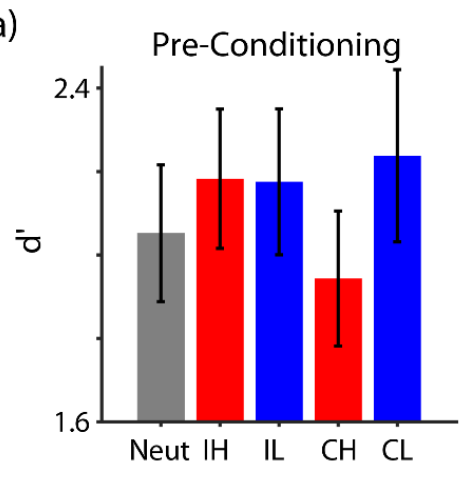

b)

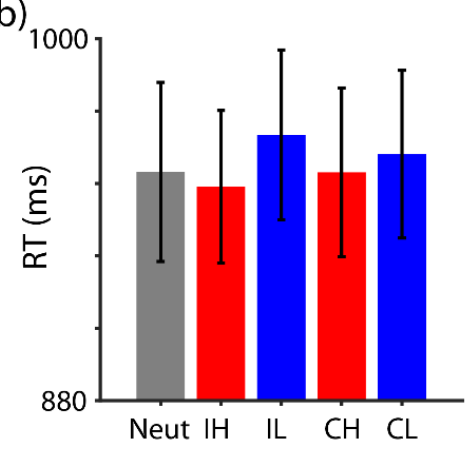

c)

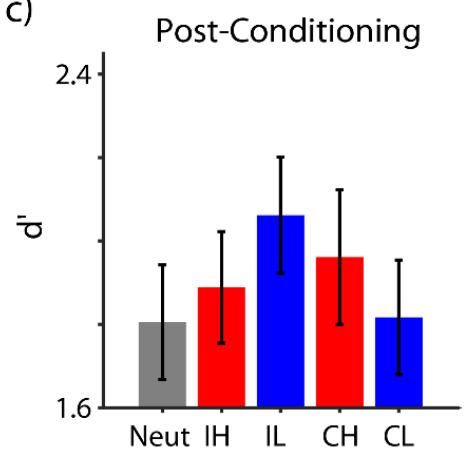

d)

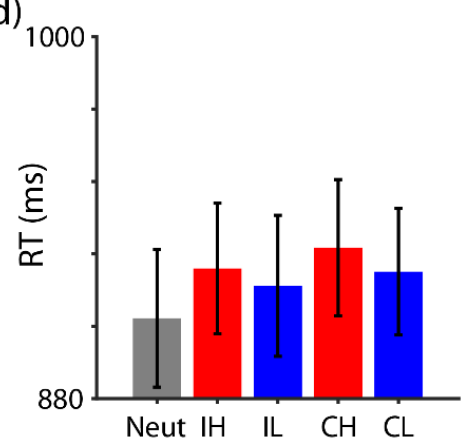

e)

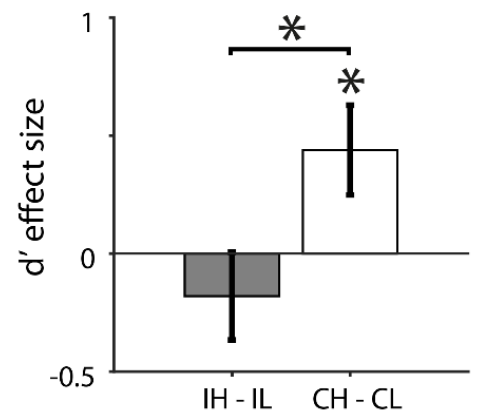

f)

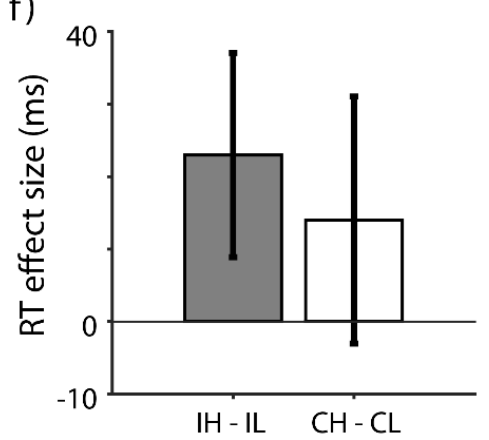

Figure 3. Behavioral performance in the orientation discrimination task. a) Visual sensitivity (d-prime: d') during the pre-conditioning phase for different conditions (Neutral: Neut; Intra-modal High Value: IH; Intra-Modal Low Value: IL; Cross-modal High Value: CH; and Cross-Modal Low Value: CL). b) Same as a, for reaction times (RT). c) Visual sensitivity during the post-conditioning phase, i.e. after the associative reward value of different cues were learned. d) Same as $\mathbf{c}$ for RTs. e) Effect size for d' modulations, measured as the difference in $\mathrm{d}^{\prime}$ between high- and low-value conditions corrected for their difference during pre-conditioning, in intra- and cross-modal cue types (grey and white bars, respectively). f) Same as e for reaction times (RT). * marks the significant effects $(\mathrm{p}<0.05)$. Error bars are s.e.m.

\section{ERP responses in posterior $R O I$}

We next examined the responses of the posterior ROI to the Gabor stimuli when it appeared together with task-irrelevant visual or auditory cues during the orientation discrimination task (Figure 4, Figure 5, and Table 2). Examination of P1 amplitudes with a two-way rmANOVA revealed a main effect of modality $\left(\mathrm{F}_{(1,35)}=5.65, p=0.023, \eta_{\mathrm{p}}{ }^{2}=\right.$ 0.14), corresponding to larger P1 amplitudes in the cross-modal condition than in the intramodal condition (see Table 2). The main effect of reward value $\left(\mathrm{F}_{(1,35)}<1, p=0.831\right)$ and the interaction between reward value and modality did not reach significance $\left(\mathrm{F}_{(1,35)}<1, p\right.$ $=0.501)$. Analysis of P1 latencies did not reveal any effect of modality $\left(\mathrm{F}_{(1,35)}<1, p=\right.$ $0.935)$, value $\left(\mathrm{F}_{(1,35)}<1, p=0.342\right)$, or their interaction $\left(\mathrm{F}_{(1,35)}=1.2, p=0.287\right)$.

Analysis of the N1 amplitude revealed a trend for an effect of modality $\left(\mathrm{F}_{(1,35)}=3.6\right.$, $p=0.066, \eta_{\mathrm{p}}^{2}=0.093$ ), with intra-modal reward cues evoking less negative $\mathrm{N} 1$ peak than cross-modal reward cues (see Table 2). The main effect of reward value did not reach significance $\left(\mathrm{F}_{(1,35)}=2.04, p=0.162\right)$ but a trend was found for an interaction between reward value and modality $\left(\mathrm{F}_{(1,35)}=4.09, p=0.051, \eta_{\mathrm{p}}{ }^{2}=0.105\right)$, where high value cross- 
modal cues decreased N1 negativity compared to intra-modal condition (see Table 2 and Figure 4-5). Planned, pairwise comparisons showed that cross-modal high value condition significantly decreased $\left(\mathrm{t}(35)=2.98, p=0.005, \mathrm{~d}_{\mathrm{z}}=0.5\right.$, Figure 5c) the $\mathrm{N} 1$ negativity compared to the low value condition. The difference between intra-modal, high and low value conditions did not reach statistical significance $(\mathrm{p}=0.45)$. Latency analysis of $\mathbf{N 1}$ component did not reveal any effects of modality $\left(\mathrm{F}_{(1,35)}<1, p=0.935\right)$, value $\left(\mathrm{F}_{(1,35)}<1, p\right.$ $=0.363)$ or their interaction $\left(\mathrm{F}_{(1,35)}=2.17, p=0.150\right)$.

Analysis of the P3 component in midline electrodes (300-600 ms, corrected for preconditioning) revealed no main effect of modality, reward value, electrode, or their interaction (all ps > 0.1). Although post-hoc inspection of the ERP responses of midline electrodes (Supplementary Figures 2 and 3) suggests that value-driven modulations occur in other time windows than our pre-registered intervals, we decided to adhere to our a priori plan and do not explore these modulations any further.

[Insert Table. 2 here]

\begin{tabular}{|c|c|c|c|c|c|c|c|c|c|c|}
\hline & \multicolumn{5}{|c|}{ Pre-conditioning } & \multicolumn{5}{|c|}{ Post-conditioning } \\
\hline & $\mathrm{IH}$ & IL & $\mathrm{CH}$ & $\mathrm{CL}$ & Neut & $\mathrm{IH}$ & IL & $\mathrm{CH}$ & $\mathrm{CL}$ & Neut \\
\hline $\begin{array}{l}\text { RT } \\
(\mathbf{m s})\end{array}$ & $\begin{array}{l}950 \\
\pm 25.3\end{array}$ & $\begin{array}{l}968 \\
\pm 28.2\end{array}$ & $\begin{array}{r}955 \\
\pm 28\end{array}$ & $\begin{array}{l}961 \\
\pm 27.8\end{array}$ & $\begin{array}{l}955 \\
\pm 29.7\end{array}$ & $\begin{array}{l}923 \\
\pm 21.7\end{array}$ & $\begin{array}{l}917 \\
\pm 23.4\end{array}$ & $\begin{array}{l}930 \\
\pm 22.6\end{array}$ & $\begin{array}{l}922 \\
\pm 20.9\end{array}$ & $\begin{array}{l}906 \\
\pm 22.8\end{array}$ \\
\hline d' $^{\prime}$ & $\begin{array}{l}2.18 \\
\pm 0.17\end{array}$ & $\begin{array}{l}2.18 \\
\pm 0.18\end{array}$ & $\begin{array}{l}1.94 \\
\pm 0.16\end{array}$ & $\begin{array}{l}2.24 \\
\pm 0.21\end{array}$ & $\begin{array}{l}2.05 \\
\pm 0.16\end{array}$ & $\begin{array}{l}1.89 \\
\pm 0.13\end{array}$ & $\begin{array}{l}2.06 \\
\pm 0.14\end{array}$ & $\begin{array}{l}1.96 \\
\pm 0.16\end{array}$ & $\begin{array}{l}1.82 \\
\pm 0.14\end{array}$ & $\begin{array}{l}1.81 \\
\pm 0.14\end{array}$ \\
\hline $\begin{array}{l}\mathbf{P A} \\
(\mu \mathrm{V})\end{array}$ & $\begin{array}{l}0.62 \\
\pm 0.29 \\
\end{array}$ & $\begin{array}{l}0.16 \\
\pm 0.26\end{array}$ & $\begin{array}{l}3.52 \\
\pm 0.40\end{array}$ & $\begin{array}{l}3.89 \\
\pm 0.41\end{array}$ & $\begin{array}{l}0.09 \\
\pm 0.24\end{array}$ & $\begin{array}{l}0.09 \\
\pm 0.15\end{array}$ & $\begin{array}{l}\mathbf{0 . 3 9} \\
\pm 0.17\end{array}$ & $\begin{array}{l}3.36 \\
\pm 0.34\end{array}$ & $\begin{array}{l}3.30 \\
\pm 0.36\end{array}$ & $\begin{array}{l}0.40 \\
\pm 0.17\end{array}$ \\
\hline $\begin{array}{l}\mathbf{P 1} \\
(\mu \mathrm{V})\end{array}$ & $\begin{array}{l}2.28 \\
\pm 0.36\end{array}$ & $\begin{array}{l}2.35 \\
\pm 0.35\end{array}$ & $\begin{array}{l}5.56 \\
\pm 0.43\end{array}$ & $\begin{array}{l}6.03 \\
\pm 0.39\end{array}$ & $\begin{array}{l}1.98 \\
\pm 0.34\end{array}$ & $\begin{array}{l}1.84 \\
\pm 0.26\end{array}$ & $\begin{array}{l}2.07 \\
\pm 0.30\end{array}$ & $\begin{array}{l}4.81 \\
\pm 0.34\end{array}$ & $\begin{array}{l}5.00 \\
\pm 0.36\end{array}$ & $\begin{array}{l}1.93 \\
\pm 0.26\end{array}$ \\
\hline $\begin{array}{l}\text { N1 } \\
(\mu \mathrm{V})\end{array}$ & $\begin{array}{l}0.93 \\
\pm 0.41\end{array}$ & $\begin{array}{l}0.99 \\
\pm 0.39\end{array}$ & $\begin{array}{l}-1.29 \\
\pm 0.46\end{array}$ & $\begin{array}{l}-0.67 \\
\pm 0.48\end{array}$ & $\begin{array}{l}0.42 \\
\pm 0.42\end{array}$ & $\begin{array}{l}0.38 \\
\pm 0.35\end{array}$ & $\begin{array}{l}0.74 \\
\pm 0.35\end{array}$ & $\begin{array}{l}-\mathbf{- 0 . 7 0} \\
\pm 0.40\end{array}$ & $\begin{array}{l}-0.94 \\
\pm 0.41\end{array}$ & $\begin{array}{l}0.91 \\
\pm 0.36\end{array}$ \\
\hline $\begin{array}{l}\text { P3 } \\
(\mu V)\end{array}$ & $\begin{array}{l}2.65 \\
\pm 0.41\end{array}$ & $\begin{array}{l}2.70 \\
\pm 0.43\end{array}$ & $\begin{array}{l}3.44 \\
\pm 0.47\end{array}$ & $\begin{array}{l}4.23 \\
\pm 0.49\end{array}$ & $\begin{array}{l}1.98 \\
\pm 0.46\end{array}$ & $\begin{array}{l}2.58 \\
\pm 0.46\end{array}$ & $\begin{array}{l}2.48 \\
\pm 0.45\end{array}$ & $\begin{array}{c}3.91 \\
\pm 0.47\end{array}$ & $\begin{array}{l}3.76 \\
\pm 0.49\end{array}$ & $\begin{array}{l}2.52 \\
\pm 0.45\end{array}$ \\
\hline $\begin{array}{l}\text { P1 } \\
\text { (ms) }\end{array}$ & $\begin{array}{l}151 \\
\pm 3.63\end{array}$ & $\begin{array}{l}152 \\
\pm 3.78\end{array}$ & $\begin{array}{l}141 \\
\pm 3.08\end{array}$ & $\begin{array}{l}145 \\
\pm 2.78\end{array}$ & $\begin{array}{l}147 \\
\pm 4.15\end{array}$ & $\begin{array}{l}147 \\
\pm 4.01\end{array}$ & $\begin{array}{l}147 \\
\pm 4.33\end{array}$ & $\begin{array}{c}140 \\
\pm 3.62\end{array}$ & $\begin{array}{l}136 \\
\pm 3.50\end{array}$ & $\begin{array}{l}151 \\
\pm 3.26\end{array}$ \\
\hline $\begin{array}{l}\text { N1 } \\
\text { (ms) }\end{array}$ & $\begin{array}{l}214 \\
\pm 3.85\end{array}$ & $\begin{array}{l}213 \\
\pm 3.71\end{array}$ & $\begin{array}{l}214 \\
\pm 2.82\end{array}$ & $\begin{array}{l}213 \\
\pm 2.81\end{array}$ & $\begin{array}{l}211 \\
\pm 3.59\end{array}$ & $\begin{array}{l}209 \\
\pm 3.29\end{array}$ & $\begin{array}{l}215 \\
\pm 3.34\end{array}$ & $\begin{array}{c}213 \\
\pm 2.66\end{array}$ & $\begin{array}{l}210 \\
\pm 2.33\end{array}$ & $\begin{array}{l}212 \\
\pm 3.65\end{array}$ \\
\hline
\end{tabular}

Table 2. Summary of behavioral and ERP data during the pre- and post-conditioning phases (the gray and white cells, respectively) for intra-modal (IH and IL, high and low values), cross-modal (CH and CI, high and low value), and neutral (Neut) conditions. Significant pairwise comparisons are shown in bold fonts $(\mathrm{p}<0.05)$.

Based on our pre-registered analysis plan, we next examined the earliest and latest time points, where the reward modulations of each cue type were significant using a moving window analysis (see Methods). This analysis revealed an earlier onset of value-driven modulations for intra-modal compared to cross-modal conditions in the posterior ROI. Highand low-value ERP responses of the intra-modal conditions differed significantly within the P1 window, i.e., between 101 and 114 ms. The cross-modal value-modulations started later, at $167 \mathrm{~ms}$, and remained significant until $223 \mathrm{~ms}$, thus overlapping with the N1 time window 
(Figure 4). Based on these results and visual inspection of the ERPs, we performed an exploratory analysis on the amplitude of evoked responses in a time window between 90$120 \mathrm{~ms}$, where early value-driven modulation was found for intra-modal conditions. This amplitude modulation occurred earlier than our pre-registered measure of P1 component and will be referred to as the PA component throughout. A two-way ANOVA on PA amplitudes revealed a significant value $\times$ modality interaction effect $\left(\mathrm{F}_{(1,35)}=5.12, p=0.03, \eta_{\mathrm{p}}{ }^{2}=0.128\right)$, but no main effect of reward value or modality (both Fs $<1$ and ps $>0.1$ ). Post-hoc pairwise comparisons (Figure 5c) revealed a significant suppression of PA amplitude for highcompared to low-value intra-modal condition $\left(\mathrm{t}(35)=-2.1, p=0.04, \mathrm{~d}_{\mathrm{z}}=0.35\right)$. The valuedriven modulation in the cross-modal conditions was in opposite direction but did not reach statistical significance $\left(\mathrm{t}(35)=1.35, p=0.185, \mathrm{~d}_{\mathrm{z}}=0.22\right)$. We wondered whether the negative modulation of PA responses by intra-modal reward value reflects a suppression of high-reward stimuli or rather an enhancement of the low-reward conditions. To this end, we contrasted the PA amplitudes of intra-modal high- and low-value conditions, respectively, against the neutral condition that had not undergone reward associative learning (see also Figure 4-5). These comparisons revealed a significant suppression of PA responses evoked by intra-modal, high-value compared to neutral stimuli $\left(\mathrm{t}(35)=-2.07, p=0.044, \mathrm{~d}_{\mathrm{z}}=0.35\right)$. The intra-modal, low-value stimuli, however, were not significantly different from the neutral condition $\left(\mathrm{t}(35)=0.25, p=0.801, \mathrm{~d}_{\mathrm{z}}=0.042\right)$. These results suggest that intra-modal high-value stimuli undergo active suppression compared to both intra-modal low value and neutral conditions occurring very early after the onset of stimuli. 

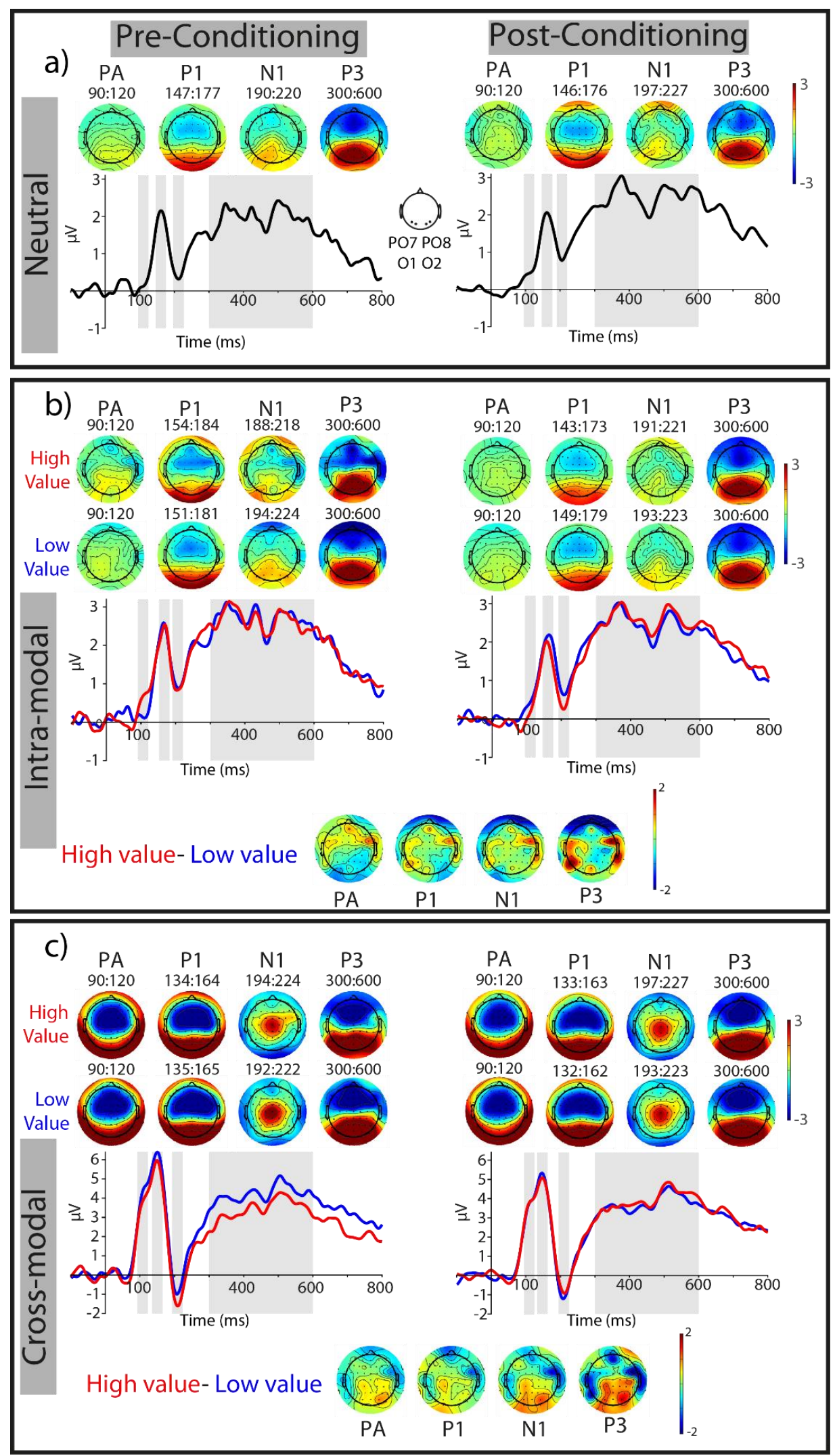

Figure 4. ERP responses of the posterior ROI (PO7, PO8, O1, O2) during the visual orientation discrimination task. a) ERPs elicited by the neutral condition during pre- and post-conditioning (illustrated on the left and right, respectively). The topographic distributions of PA (90-120 ms), P1 (most positive peak 70-170 ms), N1 (most negative peak 180-250 ms, and P300 (300-600 ms) components, measured in respective grey shaded areas of the ERP time courses are also shown. b) ERPs in the presence of task-irrelevant, intramodal reward cues, with high (red traces) and low (blue traces) values. The corresponding topographic distribution of each component is shown for each reward value condition. To test the significance of value- 
driven modulations, the difference between high- and low-value conditions was corrected for their preconditioning difference. The topographic distributions of these corrected modulations are shown for each component (the lowermost topographic maps). See also Figure 5c. c) Same as $\mathbf{b}$ for ERPs in the presence of task-irrelevant, cross-modal reward cues.

We next performed an exploratory analysis on the ERPs of the posterior ROI in a later time window (300-600 ms) corresponding to the $P 3$ component. This analysis revealed a main effect of reward value $\left(\mathrm{F}_{(1,35)}=5.63, p=0.023, \eta_{\mathrm{p}}^{2}=0.138\right)$. The main effect of modality and the modality by reward interaction were not significant (both ps > 0.1). Planned, pairwise comparisons revealed a significant enhancement of P3 responses by crossmodal high- compared to low-value condition $(\mathrm{t}(35)=2.72, \mathrm{P}=0.01, \mathrm{~d}=0.45)$. In the intramodal condition, P3 amplitudes did not differ between the two reward conditions $(\mathrm{t}(35)=$ $\left.0.37, p=0.72, \mathrm{~d}_{\mathrm{z}}=0.061\right)$. Together, examination of late ERPs in the posterior ROI mainly indicated a value-driven response enhancement for cross-modal condition (Figure 5c).

In our pre-registered analysis plan, we had intended to compare the ERP effects of the correct and error trials. However, after the data acquisition, we noticed that we could not have a noise-free estimation of the ERPs of error trials, as in some cases errors were too infrequent (Mean \pm SD: $6.6 \pm 2.4$ and $13.6 \pm 5.4$, for number of error trials in pre- and postconditioning, respectively). Therefore, we did not undertake this pre-registered analysis, albeit we tested whether we obtain similar results when we only include correct trials in our analysis (for which we had sufficient number of trials; i.e.,> 25 trials for each condition and each phase). Our analysis showed that overall the pattern of results remains unchanged when only correct trials were included in our analyses and in most cases effect sizes were larger than when all trials were included in our analysis (Supplementary Text and Supplementary Figure 5).

Finally, due to the retinotopic organization of visual cortex, responses to lateralized stimuli are strongest in contralateral cortical regions; therefore, we tested potential valuedriven modulations of ERPs of each condition in contra-lateral posterior ROIs separately (Supplementary Figure 4). Results from these analyses were highly similar to those, where ERPs in the posterior ROI were averaged across both hemispheres (see also the Supplementary Information).

Taken together, the ERP effects provide evidence for an interaction effect between intra- and cross-modal reward cues, with intra-modal cues leading to an early suppression and cross-modal reward cues producing a later response enhancement for high-compared to low-value stimuli. 
a)

PA

P1

N1

P3

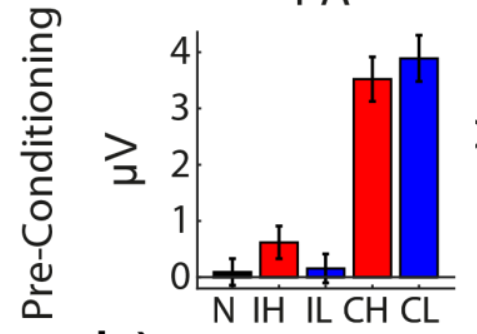

b)
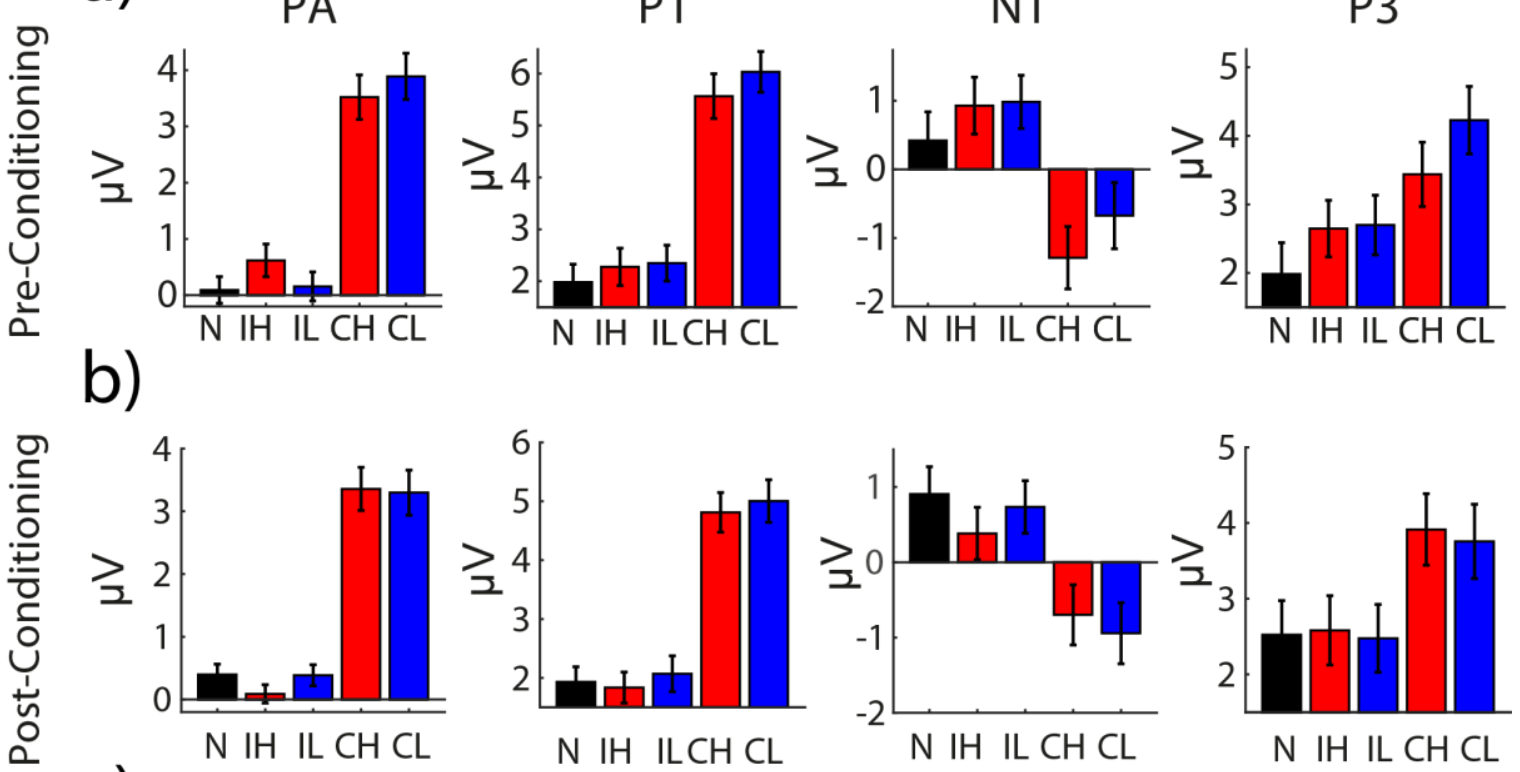

C)
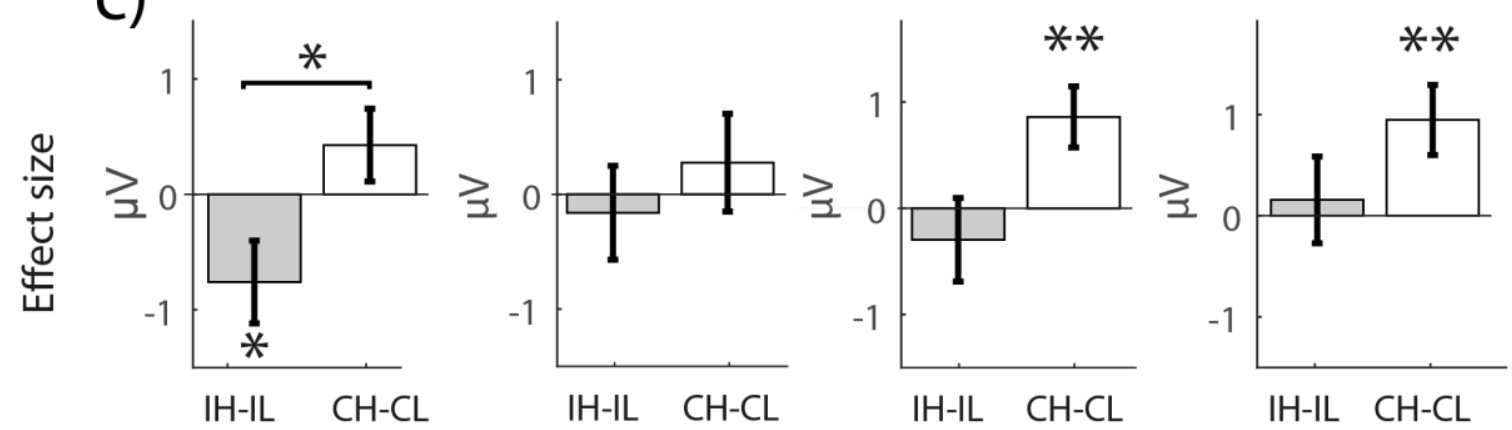

Figure 5. Mean amplitude of different ERP components of the posterior ROI (PO7, PO8, O1, O2) during the visual orientation discrimination task. a) mean amplitude of PA, P1, N1, and P3 responses evoked by neutral $(\mathrm{N})$, intra-modal high value (IH), intra-modal low value (IL), cross-modal high value (CH) and crossmodal low value (CL) conditions, during pre-condoning. b) Same as a, for ERPs during post-condoning. c) Corrected effect sizes, measured as the difference between high- and low-value conditions corrected for their difference during pre-conditioning, for intra- and cross-modal cue types (grey and white bars, respectively). * mark significant differences $(\mathrm{p}<0.05)$ and $* *$ mark significant differences $(\mathrm{p}<0.01)$. Error bars are s.e.m.

\section{Correlation of behavioral and ERP effects}

We next measured the correlation between value-driven amplitude modulations of components for which a significant reward effect was found (i.e., PA, N1, and P3, see Figure 5) and changes in behavioral indices (d' and RT). This analysis did not reveal any significant correlation (all ps>0.1). We wondered whether the lack of a correlation with behavior, particularly with $\mathrm{d}^{\prime}$ is due to averaging the responses of contralateral and ipsilateral electrodes in our ROI. Due to the strong retinotopic organization of early visual areas, the reward modulations of lateralized stimulus are strongest and most reliably correlated with behavior on the contralateral visual areas (Pooresmaeili et al., 2014). To this end, we performed an exploratory analysis where the responses of the contralateral electrodes of the posterior ROI measured in each time window (corresponding to PA, N1, and P3) were 
correlated with the behavioral d' of the corresponding condition. This analysis revealed a significant positive correlation between value-driven modulations of the contralateral ERPs in $\mathrm{N} 1\left(\mathrm{r}=0.347, \mathrm{p}=0.037\right.$, Pearson Correlation, $\left.\mathrm{d}_{\mathrm{z}}=0.74\right)$ and $\mathrm{P} 3(\mathrm{r}=0.352, \mathrm{p}=0.035$, Pearson Correlation, $\mathrm{d}_{\mathrm{z}}=0.75$ ) windows and behavioral d-primes of the cross-modal stimuli. These results hence indicate that the cross-modal reward effects on behavior may indeed rely on the value-driven response modulations of the contralateral visual areas. However, we note that these results should be treated with caution, as they reflect results obtained from an exploratory analysis and were not corrected for multiple comparisons.

[Insert figure 6]
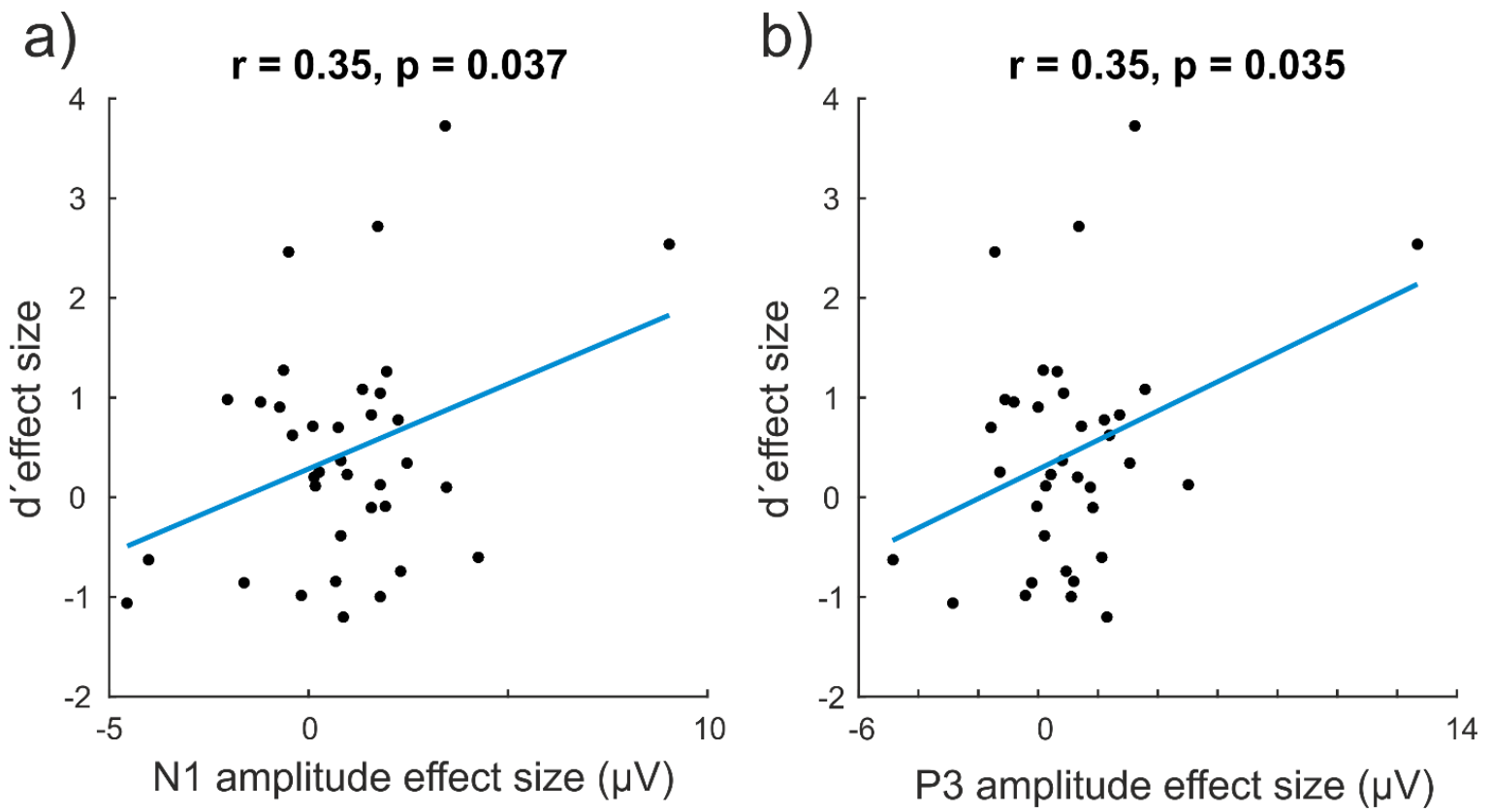

Figure 6. Correlation between electrophysiological and behavioral effects of reward value. a) Correlation between reward modulation of contralateral N1 amplitude and d' in cross-modal condition. Effect size corresponds to the difference of high- and low-value conditions corrected for their difference during preconditioning. b) Same as a for the contralateral P3 amplitude. 


\subsection{Discussion}

In the current study we tested whether intra-modal and cross-modal reward cues exert similar effects on the processing of visual stimuli. To this end, we examined behavioral and electrophysiological responses to reward cues during a conditioning phase when reward associations were learned and during a post-conditioning phase when rewards were not delivered anymore. Importantly, during post-conditioning reward cues were irrelevant to the visual discrimination task that participants had to perform. In the conditioning phase, we found that intra- and cross-modal reward cues affect latency of the N1 responses over the posterior electrodes differently: while intra-modal reward cues significantly sped up N1 response, there was a trend for deceleration of $\mathrm{N} 1$ responses by cross-modal reward cues. However, in the P3 component, we found higher amplitudes for both intra- and cross-modal high-value cues. In the post-conditioning phase, similarly to the conditioning phase, P3-like responses of the posterior ROI were enhanced for high-value cues of both types. Importantly, we found much earlier reward effects within 90-120 ms (P1 window), where intra-modal high-value reward cues suppressed ERP responses compared to the low-value reward cues. The reward effects from cross-modal cues, on the other hand, led to a later modulation at a time point corresponding to the N1 component. Behavioral results were in line with electrophysiological findings; we found an interaction between the reward value and the modality of the reward cue: cross-modal reward cues significantly enhanced visual sensitivity whereas intra-modal reward cues showed a trend for suppression. Interaction effects of reward value with modality within P1 and N1 windows suggest that intra-modal and cross-modal reward values exert different effects on visual perception under settings employed in our study (i.e., task-irrelevant reward cues during a no-reward phase). Similar reward effects of intra- and cross-modal conditions in the P3 window indicate that beyond the differential effect of modality on early visual responses, later reward effects are independent of sensory modality. In the following sections, we will discuss possible mechanisms that might explain such interaction effects at the early ERP components.

\section{Reward-driven modulations during conditioning}

In this phase, a visual target predictive of higher rewards sped up reaction times and early cue-evoked neural responses, particularly in the N1 window. This result is similar to early modulations of visual ERPs (i.e., <250 ms) observed in previous studies (Baines et al., 2011; Hammerschmidt et al., 2018; Hickey et al., 2010b; Luque et al., 2017, 2015; Maclean and Giesbrecht, 2015). Auditory stimuli also evoked strong responses in the visual cortex even 
in the absence of concomitant visual stimulation (Feng et al., 2014; McDonald et al., 2013; Weinberger, 2007; Wikman et al., 2019). However, reward signals from auditory stimuli did not significantly affect the amplitude or latency of early visual ERP components when auditory stimuli were presented alone, albeit a trend was found for deceleration of N1 responses. Lack of cross-modal reward effects during the conditioning phase may indicate that reward information does not transfer automatically when there is no incoming visual information. A trend for deceleration of $\mathrm{N} 1$ responses and reaction times by cross-modal reward cues may also be due to the type of the task employed during conditioning (i.e., a localization task) as auditory modality is generally less sensitive to spatial properties of the stimuli than vision (Bertelson and Radeau, 1981; Pick et al., 1969).

The similarity of intra- and cross-modal reward effects on later P3 component indicates that at the later stages of sensory processing, reward information integrates into a coherent reward representation across various sources irrespective of the unique characteristics of those sources. On the other hand, during the early stages of sensory processing, privileged processing of rewarded stimuli only prioritizes cues that are most suited for the task at hand, i.e., vision in case of a task requiring localization. These results are overall explainable within the framework of reinforcement learning, where association of a stimulus with reward results in value-driven modulations at 2 time points (Luque et al., 2015): an early modulation of neural responses for stimuli associated with higher value within the first 200-250 ms after the stimulus onset (Luque et al., 2015; Maclean and Giesbrecht, 2015); and a later reward modulation primarily the P3 component related to anticipation of the reward delivery (Goldstein et al., 2006; Luque et al., 2017, 2015; Pornpattananangkul and Nusslock, 2015; Yeung and Sanfey, 2004).

\section{Reward-driven modulations during post-conditioning}

The results obtained in the post-conditioning phase showed a differential pattern of reward-related modulation for cross-modal and intra-modal cues. Specifically, we observed an improvement of behavioral measures of visual sensitivity for high compared to low-value cross-modal cues. These results are in line with the reported facilitatory effects of crossmodal value on visual processing observed in human psychophysical and neuroimaging studies (Leo et al., 2011; Pooresmaeili et al., 2014). As a first attempt to characterize the electrophysiological correlates of this effect, our study demonstrated that the behavioral advantage conferred by high-reward auditory cues is accompanied by a modulation of visual ERPs. As these modulations were corrected for differences potentially occurring due to 
physical stimulus features (i.e., tone pitches) already during the pre-conditioning phase, they most probably reflect effects driven by associative learning of the reward values.

Cross-modal reward modulations in our study occurred later than attentional effects observed in some of the previous studies of cross-modal attention (Störmer et al., 2009), where response modulations were found in P1 window. However, in these experiments auditory tones were presented prior to the visual target and could therefore modulate the early ERP responses. In fact, when auditory and visual components of an audiovisual object were presented together, attentional effects occurred at a later time window around $220 \mathrm{~ms}$ (Busse et al., 2005; Zimmer et al., 2010). Whereas reward-driven boost of attention may to some extent account for cross-modal reward effects in our study, the direction of response modulations suggests that additional mechanisms may also be involved. Specifically, we found a reduction in N1 negativity after learning of the reward associations, which is different from an enhanced P1 positivity and N1 negativity that has been observed in studies of cross-modal attention (Busse et al., 2005; Störmer et al., 2009; Zimmer et al., 2010). One possible mechanism is a reward-driven enhancement of audiovisual integration, which is in line with recent findings demonstrating a role of reward in multisensory integration (Bean et al., 2021; Cheng et al., 2020). This mechanism can also explain the direction of ERP modulations, as previous studies found that an audiovisual stimulus elicits response modulations of visual ERPs mainly in N185 window, with a reduction of the negativity of N185 component compared to the unimodal stimuli (Giard and Peronnet, 1999), which is similar to the pattern of modulations we observed for cross-modal rewards. The reduction in $\mathrm{N} 1$ negativity may indicate that audiovisual integration enhances the gain of the neural responses of visual cortex, hence reducing the energy (i.e. N1 amplitude) needed to process the same load of sensory information, an example of sub-additive cross-modal interactions reported before (Molholm et al., 2002; Teder-Sälejärvi et al., 2005, 2002). An enhanced integration between auditory reward cues and visual targets in our task potentially reduces the distracting effect of task-irrelevant sounds on visual discrimination while promoting the spread of privileged processing from rewarded sounds to the visual target. Later modulations by cross-modal compared to intra-modal rewards suggests that a putative reward-driven enchantment of audiovisual integration may first occur in multimodal areas such as in Superior Temporal Sulcus (STS), being then fed back to visual cortex, a proposal in line with the findings of neuroimaging studies of cross-modal value and emotion effects on vision (Pooresmaeili et al., 2014; Watson et al., 2014). In addition to the above mechanisms (reward-driven boost of attention and audiovisual integration), the later value-driven 
modulations in P3 window may indicate that the cross-modal reward effects also rely on post-sensory and decisional stages (Franzen et al., 2020).

The effects of intra-modal reward cues on visual perception were contrary to our a-priori hypothesis that intra-modal and cross-modal reward stimuli should have similar facilitatory effects on visual perception. This hypothesis was based on previous findings (Stanisor et al., 2013) that reported a spread of reward enhancement effects from one component of an object to its other parts (here from colored circles signaling reward to the Gabor target), akin to the spread of object-based attention (Duncan, 1984; Pooresmaeili and Roelfsema, 2014). Contrary to our prediction, we found that intra-modal reward stimuli interfered with sensory processing of the visual target, which was reflected in suppressed ERP responses at an early time window of 90-120 ms elicited by high rewards compared to both low reward and neutral conditions. The interference effect that we observed is in line with the findings of several studies where value-driven effects during visual search were investigated (Anderson, 2013; Anderson et al., 2011b, 2011a; Chang and Egeth, 2021; Feldmann-Wüstefeld and Schubö, 2013; Gong et al., 2016; Hickey et al., 2009; Rusz et al., 2020, 2018; Yantis et al., 2012). These experiments have consistently reported that presentation of a high reward stimulus at the target location speeds up visual search and enhances target-evoked responses, whereas presentation of high reward stimuli at a the distractor location captures attention away from the target and interferes with the search task. Given these previous findings, we superimposed reward cues on the target to boost the processing of all object elements at that location. However, since the visual discrimination task was performed on a different feature of the object (i.e. orientation) than the defining feature of the reward cue (color), high reward visual cues may have captured attention away from the target feature, hence interfering with the target processing. A similar interference effect has been observed in studies where a certain feature of an object was predictive of its reward but this feature was incongruent with the goal of the task, e.g. in many tasks employing the Stroop Effect (Bustamante et al., 2021; Grégoire and Anderson, 2019; Krebs et al., 2013, 2011, 2010).

In light of these previous studies, we propose four possible mechanisms for the observed intra-modal, reward-related suppression. Firstly, it is possible that as a result of an enhanced response to the high-reward distractors (Itthipuripat et al., 2019) some form of local inhibition is exerted on the adjacent stimuli, thereby decreasing the overall responses to target and rewarding distractors that were at the same location. Such a center-surround inhibition around an attended feature has been observed in studies of feature-based and spatial attention (Hopf et al., 2006; Mounts, 2000; Störmer and Alvarez, 2014). Secondly, it 
is possible that the suppression is a reflection of the higher processing load of high-reward cues (Engelmann et al., 2009) and the capacity limitation of attentional processing. This mechanism could therefore result from a mixture of enhanced processing of distractors in some trials and decrement of processing of target+distractor in other trials due to the depletion of attentional resources. Thirdly, it is possible that the suppression is due to cognitive control mechanisms that actively inhibit the processing of intra-modal reward cues with a resultant spillover of inhibition to the target as also observed in previous studies of feature-based attention (Nobre et al., 2006). The fourth possibility is that reduction of neural responses in visual cortex is a general characteristic of reward-related modulations occurring due to direct dopaminergic inputs to visual cortex (Arsenault et al., 2013) or value-driven attentional gain control mechanisms (Shapcott et al., 2020) that adjust signal-to-noise ratio of neural responses. These scenarios may all contribute to the suppression of responses to intra-modal high reward cues that we observed, although the early onset of the suppressive effects best matches the first two scenarios. Future studies will be needed to tease apart these possibilities.

In summary, our study showed that intra- and cross-modal reward stimuli affect visual perception during a no-reward (post-conditioning) phase differently. In line with our a priori hypothesis, intra-modal reward cues affected visual sensory processing early, thus the modulation took place within the visual cortex. Cross-modal reward effects, on the other hand, occurred much later and exhibited characteristics of audiovisual integration, thus being likely to rely on feedback from multimodal areas, such as STS. We did not expect to find a difference between the direction of modulations evoked by intra- and cross-modal reward stimuli. However, ERP analysis of P1 and N1 components revealed the difference between these conditions, most importantly an early suppression of intra-modal high reward condition. We suggested a number of factors that could contribute to the latter effect: a local suppression induced by reward-irrelevant cues on adjacent target stimuli, a competition for processing resources between target and reward stimuli, active inhibition due to cognitive control mechanisms, or a general mechanism of reward modulation where dopamine as a result of high-reward stimuli adjust signal-to-noise ratio, thus suppress the neuronal response. The first two mechanisms are most favorable, but subsequent studies are needed to tease them apart. Overall, our study provides evidence for value-driven plasticity in visual perception that can be induced not only by signals from the visual modality but also crossmodally. Embedding cross-modal rewards in visual tasks is a promising tool to assist vision, 
especially in the face of visual impairments, through boosting cross-modal advantages conferred by another intact sensory modality.

\section{Acknowledgments}

We thank Adem Saglam for his help with the programming of the experiment, Franziska Ehbrecht for her help with the data collection, and Jessica Emily Antono for her valuable comments on the manuscript. This work was supported by an ERC Starting Grant (no: 716846) to AP.

\section{Authors' contributions}

RV and AP conceptualized and designed the experiment. All authors participated in the pre-registration of the study. RV conducted the experiments. RV, FC, and AP analyzed the data. RV and AP interpreted the results and wrote the first draft of the manuscript. All authors revised the manuscript. 


\subsection{References}

Ainslie, G., 1975. Specious reward: A behavioral theory of impulsiveness and impulse control. Psychol. Bull. 82, 463-496. https://doi.org/10.1037/h0076860

Alais, D., Morrone, C., Burr, D., 2006. Separate attentional resources for vision and audition. Proc. R. Soc. B Biol. Sci. 273, 1339-1345. https://doi.org/10.1098/rspb.2005.3420

Algazi, U., Duda, R., Thompson, D., The, C.A.-2001 I.W. on, 2001. The CIPIC HRTF Database, in Applications of Signal Processing to Audio and Acoustics. IEEE Work. on the, 99-102.

Alsius, A., Navarra, J., Campbell, R., Soto-Faraco, S., 2005. Audiovisual integration of speech falters under high attention demands. Curr. Biol. 15, 839-843. https://doi.org/10.1016/j.cub.2005.03.046

Anderson, B.A., 2019. Neurobiology of value-driven attention. Curr. Opin. Psychol. https://doi.org/10.1016/j.copsyc.2018.11.004

Anderson, B.A., 2017. Reward processing in the value-driven attention network: reward signals tracking cue identity and location. Soc. Cogn. Affect. Neurosci. 12, 461-467. https://doi.org/10.1093/scan/nsw141

Anderson, B.A., 2016a. Value-driven attentional capture in the auditory domain. Attention, Perception, Psychophys. 78, 242-250. https://doi.org/10.3758/s13414-015-1001-7

Anderson, B.A., 2016b. The attention habit: how reward learning shapes attentional selection. Ann. N. Y. Acad. Sci. 1369, 24-39. https://doi.org/10.1111/nyas.12957

Anderson, B.A., 2013. A value-driven mechanism of attentional selection. J. Vis. 13, 7-7. https://doi.org/10.1167/13.3.7

Anderson, B.A., Kim, A.J., 2020. Selection history-driven signal suppression. Vis. cogn. https://doi.org/10.1080/13506285.2020.1727599

Anderson, B.A., Kim, H., Kim, A.J., Liao, M.-R., Mrkonja, L., Clement, A., Grégoire, L., 2021. The Past, Present, and Future of Selection History. Neurosci. Biobehav. Rev. 130, 326-350. https://doi.org/10.1016/j.neubiorev.2021.09.004

Anderson, B.A., Laurent, P.A., Yantis, S., 2011a. Value-driven attentional capture. Proc. Natl. Acad. Sci. U. S. A. 108, 10367-10371. https://doi.org/10.1073/pnas.1104047108

Anderson, B.A., Laurent, P.A., Yantis, S., 2011b. Learned Value Magnifies Salience-Based Attentional Capture. PLoS One 6, e27926. https://doi.org/10.1371/journal.pone.0027926

Anderson, B.A., Yantis, S., 2013. Persistence of value-driven attentional capture. J. Exp. Psychol. Hum. Percept. Perform. 39, 6-9. https://doi.org/10.1037/a0030860

Anderson, Laurent, Yantis, Anderson, B.A., Laurent, P.A., Yantis, S., 2013. Reward predictions bias attentional selection. Front. Hum. Neurosci. 7, 1-6. https://doi.org/10.3389/fnhum.2013.00262

Arsenault, J.T., Nelissen, K., Jarraya, B., Vanduffel, W., 2013. Dopaminergic Reward Signals Selectively Decrease fMRI Activity in Primate Visual Cortex. Neuron 77, 1174-1186. https://doi.org/10.1016/j.neuron.2013.01.008

Asutay, E., Västfjäll, D., 2016. Auditory attentional selection is biased by reward cues. Sci. Rep. 6, 1-6. https://doi.org/10.1038/srep36989

Awh, E., Belopolsky, A. V., Theeuwes, J., 2012. Top-down versus bottom-up attentional control: A failed theoretical dichotomy. Trends Cogn. Sci. https://doi.org/10.1016/j.tics.2012.06.010

Bachman, M.D., Wang, L., Gamble, M.L., Woldorff, M.G., 2020. Physical Salience and ValueDriven Salience Operate through Different Neural Mechanisms to Enhance Attentional Selection. J. Neurosci. 40, 5455-5464. https://doi.org/10.1523/JNEUROSCI.1198-19.2020

Baines, S., Ruz, M., Rao, A., Denison, R., Nobre, A.C., 2011. Modulation of neural activity by motivational and spatial biases. Neuropsychologia 49, 2489-2497. https://doi.org/10.1016/j.neuropsychologia.2011.04.029

Balan, P.F., Gottlieb, J., 2006. Integration of exogenous input into a dynamic salience map revealed by perturbing attention. J. Neurosci. 26, 9239-9249. https://doi.org/10.1523/JNEUROSCI.1898-06.2006

Baldassi, S., Simoncini, C., 2011. Reward sharpens orientation coding independently of attention. Front. Neurosci. 5, 13. https://doi.org/10.3389/fnins.2011.00013

Bao, S., Chan, V.T., Merzenich, M.M., 2001. Cortical remodelling induced by activity of ventral 
tegmental dopamine neurons. Nature 412, 79-83. https://doi.org/10.1038/35083586

Basten, U., Biele, G., Heekeren, H.R., Fiebach, C.J., 2010. How the brain integrates costs and benefits during decision making. Proc. Natl. Acad. Sci. U. S. A. 107, 21767-21772. https://doi.org/10.1073/pnas.0908104107

Bayer, M., Grass, A., Schacht, A., 2019. Associated valence impacts early visual processing of letter strings: Evidence from ERPs in a cross-modal learning paradigm. Cogn. Affect. Behav. Neurosci. 19, 98-108. https://doi.org/10.3758/s13415-018-00647-2

Bayer, M., Rossi, V., Vanlessen, N., Grass, A., Schacht, A., Pourtois, G., 2017. Independent effects of motivation and spatial attention in the human visual cortex. Soc. Cogn. Affect. Neurosci. 12, 146-156. https://doi.org/10.1093/scan/nsw162

Bayer, M., Sommer, W., Schacht, A., 2012. P1 and beyond: Functional separation of multiple emotion effects in word recognition. Psychophysiology 49, 959-969. https://doi.org/10.1111/j.1469-8986.2012.01381.x

Bean, N.L., Stein, B.E., Rowland, B.A., 2021. Stimulus value gates multisensory integration. Eur. J. Neurosci. 53, 3142-3159. https://doi.org/10.1111/ejn.15167

Berridge, K.C., 2004. Motivation concepts in behavioral neuroscience. Physiol. Behav. 81, 179-209. https://doi.org/10.1016/J.PHYSBEH.2004.02.004

Berridge, K.C., 2000. Reward learning: Reinforcement, incentives, and expectations. Psychol. Learn. Motiv. - Adv. Res. Theory 40, 223-278.

Berridge, K.C., Kringelbach, M.L., 2015. Pleasure Systems in the Brain. Neuron 86, 646-664. https://doi.org/10.1016/J.NEURON.2015.02.018

Berridge, K.C., Kringelbach, M.L., 2008. Affective neuroscience of pleasure: Reward in humans and animals. Psychopharmacology (Berl). https://doi.org/10.1007/s00213-008-1099-6

Berridge, K.C., Robinson, T.E., 1998. What is the role of dopamine in reward: Hedonic impact, reward learning, or incentive salience? Brain Res. Rev. 28, 309-369. https://doi.org/10.1016/S0165-0173(98)00019-8

Bertelson, P., Radeau, M., 1981. Cross-modal bias and perceptual fusion with auditory-visual spatial discordance. Percept. Psychophys. 29, 578-584. https://doi.org/10.3758/BF03207374

Bisley, J.W., 2011. The neural basis of visual attention. J. Physiol. https://doi.org/10.1113/jphysiol.2010.192666

Bolognini, N., Frassinetti, F., Serino, A., Làdavas, E., 2005. “Acoustical vision” of below threshold stimuli: Interaction among spatially converging audiovisual inputs. Exp. Brain Res. 160, 273282. https://doi.org/10.1007/s00221-004-2005-Z

Bourgeois, A., Chelazzi, L., Vuilleumier, P., 2016. How motivation and reward learning modulate selective attention. Prog. Brain Res. 229, 325-342. https://doi.org/10.1016/BS.PBR.2016.06.004

Brainard, D.H., 1997. The Psychophysics Toolbox. Spat. Vis. 10, 433-436. https://doi.org/10.1163/156856897X00357

Braver, T.S., Krug, M.K., Chiew, K.S., Kool, W., Andrew Westbrook, J., Clement, N.J., Alison Adcock, R., Barch, D.M., Botvinick, M.M., Carver, C.S., Cools, R., Custers, R., Dickinson, A., Dweck, C.S., Fishbach, A., Gollwitzer, P.M., Hess, T.M., Isaacowitz, D.M., Mather, M., Murayama, K., Pessoa, L., Samanez-Larkin, G.R., Somerville, L.H., 2014. Mechanisms of motivation-cognition interaction: Challenges and opportunities. Cogn. Affect. Behav. Neurosci. https://doi.org/10.3758/s13415-014-0300-0

Bruns, P., 2019. The Ventriloquist Illusion as a Tool to Study Multisensory Processing: An Update. Front. Integr. Neurosci. https://doi.org/10.3389/fnint.2019.00051

Bruns, P., Maiworm, M., Röder, B., 2014. Reward expectation influences audiovisual spatial integration. Attention, Perception, Psychophys. 76, 1815-1827. https://doi.org/10.3758/s13414-014-0699-y

Busse, L., Roberts, K.C., Crist, R.E., Weissman, D.H., Woldorff, M.G., 2005. The spread of attention across modalities and space in a multisensory object. Proc. Natl. Acad. Sci. U. S. A. 102, 18751-18756. https://doi.org/10.1073/pnas.0507704102

Bustamante, L., Lieder, F., Musslick, S., Shenhav, A., Cohen, J., 2021. Learning to Overexert Cognitive Control in a Stroop Task. Cogn. Affect. Behav. Neurosci. 1-19. https://doi.org/10.3758/s13415-020-00845-x

Calabresi, P., Picconi, B., Tozzi, A., Di Filippo, M., 2007. Dopamine-mediated regulation of 
corticostriatal synaptic plasticity. Trends Neurosci. https://doi.org/10.1016/j.tins.2007.03.001

Calvert, G.A., Thesen, T., 2004. Multisensory integration: Methodological approaches and emerging principles in the human brain. J. Physiol. Paris 98, 191-205. https://doi.org/10.1016/j.jphysparis.2004.03.018

Carrasco, M., 2011. Visual attention: The past 25 years. Vision Res. 51, 1484-1525. https://doi.org/10.1016/J.VISRES.2011.04.012

Castiello, U., Umiltà, C., 1992. Splitting Focal Attention. J. Exp. Psychol. Hum. Percept. Perform. 18, 837-848. https://doi.org/10.1037/0096-1523.18.3.837

Chang, S., Egeth, H.E., 2021. Can salient stimuli really be suppressed? Attention, Perception, Psychophys. 83, 260-269. https://doi.org/10.3758/s13414-020-02207-8

Chelazzi, L., Eštočinová, J., Calletti, R., Gerfo, E. Lo, Sani, I., Libera, C. Della, Santandrea, E., 2014. Altering spatial priority maps via reward-based learning. J. Neurosci. 34, 8594-8604. https://doi.org/10.1523/JNEUROSCI.0277-14.2014

Chelazzi, L., Perlato, A., Santandrea, E., Della Libera, C., 2013. Rewards teach visual selective attention. Vision Res. 85, 58-72. https://doi.org/10.1016/J.VISRES.2012.12.005

Cheng, Saglam, A., André, S., Pooresmaeili, A., 2020. Cross-Modal Integration of Reward Value during Oculomotor Planning. eneuro 7, ENEURO.0381-19.2020. https://doi.org/10.1523/ENEURO.0381-19.2020

Corbetta, M., Miezin, F., Dobmeyer, S., Shulman, G., Petersen, S., 1990. Attentional modulation of neural processing of shape, color, and velocity in humans. Science (80-. ). 248, 1556-1559. https://doi.org/10.1126/SCIENCE.2360050

Corbetta, M., Shulman, G.L., 2002. Control of goal-directed and stimulus-driven attention in the brain. Nat. Rev. Neurosci. 3, 201-215. https://doi.org/10.1038/nrn755

Cornelio, P., Velasco, C., Obrist, M., 2021. Multisensory Integration as per Technological Advances: A Review. Front. Neurosci. https://doi.org/10.3389/fnins.2021.652611

Cox, S.M.L., Andrade, A., Johnsrude, I.S., 2005. Learning to like: A role for human orbitofrontal cortex in conditioned reward. J. Neurosci. 25, 2733-2740. https://doi.org/10.1523/JNEUROSCI.3360-04.2005

Dawkins, R., 2016. The selfish gene.

Della Libera, C., Chelazzi, L., 2009. Learning to Attend and to Ignore Is a Matter of Gains and Losses. Psychol. Sci. 20, 778-784. https://doi.org/10.1111/j.1467-9280.2009.02360.x

Della Libera, C., Chelazzi, L., 2006. Visual Selective Attention and the Effects of Monetary Rewards. Psychol. Sci. 17, 222-227. https://doi.org/10.1111/j.1467-9280.2006.01689.x

Delorme, A., Makeig, S., 2004. EEGLAB: An open source toolbox for analysis of single-trial EEG dynamics including independent component analysis. J. Neurosci. Methods. https://doi.org/10.1016/j.jneumeth.2003.10.009

Desimone, R., Duncan, J., 1995. NEURAL MECHANISMS OF SELECTIVE VISUAL ATTENTION, Annu. Rev. Neurosci.

Dickinson, A., Balleine, B., 1994. Motivational control of goal-directed action. Anim. Learn. Behav. 1994221 22, 1-18. https://doi.org/10.3758/BF03199951

Diederich, A., Colonius, H., 1987. Intersensory facilitation in the motor component? - A reaction time analysis. Psychol. Res. 49, 23-29. https://doi.org/10.1007/BF00309199

Driver, J., Noesselt, T., 2008. Multisensory Interplay Reveals Crossmodal Influences on "SensorySpecific" Brain Regions, Neural Responses, and Judgments. Neuron. https://doi.org/10.1016/j.neuron.2007.12.013

Duncan, J., 1984. Selective attention and the organization of visual information. J. Exp. Psychol. Gen. 113, 501-517. https://doi.org/10.1037/0096-3445.113.4.501

Duncan, J., Martens, S., Ward, R., 1997. Restricted attentional capacity within but not between sensory modalities. Nature 387, 808-810. https://doi.org/10.1038/42947

Dundon, N.M., Bertini, C., Làdavas, E., Sabel, B.A., Gall, C., 2015. Visual rehabilitation: Visual scanning, multisensory stimulation and vision restoration trainings. Front. Behav. Neurosci. https://doi.org/10.3389/fnbeh.2015.00192

Engelmann, J.B., Damaraju, E., Padmala, S., Pessoa, L., 2009. Combined effects of attention and motivation on visual task performance: Transient and sustained motivational effects. Front. Hum. Neurosci. 3, 4. https://doi.org/10.3389/neuro.09.004.2009

Eriksen, B.A., Eriksen, C.W., 1974. Effects of noise letters upon the identification of a target letter 
in a nonsearch task. Percept. Psychophys. 1974 161 16, 143-149. https://doi.org/10.3758/BF03203267

Etzel, J.A., Cole, M.W., Zacks, J.M., Kay, K.N., Braver, T.S., 2016. Reward Motivation Enhances Task Coding in Frontoparietal Cortex. Cereb. Cortex 26, 1647-1659. https://doi.org/10.1093/cercor/bhu327

Failing, M., Nissens, T., Pearson, D., Le Pelley, M., Theeuwes, J., 2015. Oculomotor capture by stimuli that signal the availability of reward. J. Neurophysiol. 114, 2316-2327. https://doi.org/10.1152/jn.00441.2015

Failing, M., Theeuwes, J., 2018. Selection history: How reward modulates selectivity of visual attention. Psychon. Bull. Rev. 25, 514-538. https://doi.org/10.3758/s13423-017-1380-y

Failing, M., Theeuwes, J., 2017. Don't let it distract you: how information about the availability of reward affects attentional selection. Attention, Perception, Psychophys. 79, 2275-2298. https://doi.org/10.3758/s13414-017-1376-8

Failing, M.F., Theeuwes, J., 2015. Nonspatial attentional capture by previously rewarded scene semantics. Vis. cogn. 23. https://doi.org/10.1080/13506285.2014.990546

Faul, F., Erdfelder, E., Lang, A.G., Buchner, A., 2007. G*Power 3: A flexible statistical power analysis program for the social, behavioral, and biomedical sciences, in: Behavior Research Methods. Psychonomic Society Inc., pp. 175-191. https://doi.org/10.3758/BF03193146

Feldmann-Wüstefeld, T., Schubö, A., 2013. Context homogeneity facilitates both distractor inhibition and target enhancement. J. Vis. 13. https://doi.org/10.1167/13.3.11

Feng, W., Stormer, V.S., Martinez, A., McDonald, J.J., Hillyard, S.A., 2014. Sounds Activate Visual Cortex and Improve Visual Discrimination. J. Neurosci. 34, 9817-9824. https://doi.org/10.1523/JNEUROSCI.4869-13.2014

Floresco, S.B., Magyar, O., 2006. Mesocortical dopamine modulation of executive functions: Beyond working memory. Psychopharmacology (Berl). https://doi.org/10.1007/s00213-0060404-5

Fox, C.R., Poldrack, R.A., 2009. Prospect theory and the brain, in: Neuroeconomics. Elsevier Inc., pp. 145-173. https://doi.org/10.1016/B978-0-12-374176-9.00011-7

Franzen, L., Delis, I., De Sousa, G., Kayser, C., Philiastides, M.G., 2020. Auditory information enhances post-sensory visual evidence during rapid multisensory decision-making. Nat. Commun. 11, 1-14. https://doi.org/10.1038/s41467-020-19306-7

Frassinetti, F., Bolognini, N., Làdavas, E., 2002. Enhancement of visual perception by crossmodal visuo-auditory interaction. Exp. Brain Res. 147, 332-343. https://doi.org/10.1007/s00221-002$1262-\mathrm{y}$

Frederick, S., Loewenstein, G., O’Donoghue, T., 2002. Time Discounting and Time Preference: A Critical Review. J. Econ. Lit. 40, 351-401. https://doi.org/10.1257/002205102320161311

Frens, M.A., Van Opstal, A.J., Van Der Willigen, R.F., 1995. Spatial and temporal factors determine auditory-visual interactions in human saccadic eye movements. Percept. Psychophys. 57, 802816. https://doi.org/10.3758/BF03206796

Friston, K., 2010. The free-energy principle: a unified brain theory? Nat. Rev. Neurosci. 2010112 11, 127-138. https://doi.org/10.1038/nrn2787

Garcia-Lazaro, H.G., Bartsch, M. V., Boehler, C.N., Krebs, R.M., Donohue, S.E., Harris, J.A., Schoenfeld, M.A., Hopf, J.M., 2018. Dissociating reward- and attention-driven biasing of global feature-based selection in human visual cortex. J. Cogn. Neurosci. 31, 469-481. https://doi.org/10.1162/jocn_a_01356

Giard, M.H., Peronnet, F., 1999. Auditory-visual integration during multimodal object recognition in humans: A behavioral and electrophysiological study. J. Cogn. Neurosci. 11, 473-490. https://doi.org/10.1162/089892999563544

Gold, J.I., Shadlen, M.N., 2007. The Neural Basis of Decision Making. Annu. Rev. Neurosci. 30, 535-574. https://doi.org/10.1146/annurev.neuro.29.051605.113038

Goldring, J.E., Dorris, M.C., Corneil, B.D., Ballantyne, P.A., Munoz, D.P., 1996. Combined eyehead gaze shifts to visual and auditory targets in humans. Exp. Brain Res. 111, 68-78. https://doi.org/10.1007/BF00229557

Goldstein, R.Z., Cottone, L.A., Jia, Z., Maloney, T., Volkow, N.D., Squires, N.K., 2006. The effect of graded monetary reward on cognitive event-related potentials and behavior in young healthy adults. Int. J. Psychophysiol. 62, 272-279. https://doi.org/10.1016/j.ijpsycho.2006.05.006 
Goltstein, P.M., Coffey, E.B.J., Roelfsema, P.R., Pennartz, C.M.A., 2013. In vivo two-photon Ca2+ imaging reveals selective reward effects on stimulus-specific assemblies in mouse visual cortex. J. Neurosci. 33, 11540-11555. https://doi.org/10.1523/JNEUROSCI.1341-12.2013

Goltstein, P.M., Meijer, G.T., Pennartz, C.M.A., 2018. Conditioning sharpens the spatial representation of rewarded stimuli in mouse primary visual cortex. Elife 7. https://doi.org/10.7554/eLife.37683

Gong, M., Yang, F., Li, S., 2016. Reward association facilitates distractor suppression in human visual search. Eur. J. Neurosci. 43, 942-953. https://doi.org/10.1111/ejn.13174

Grabenhorst, F., Rolls, E.T., Margot, C., 2011. A hedonically complex odor mixture produces an attentional capture effect in the brain. Neuroimage 55, 832-843. https://doi.org/10.1016/j.neuroimage.2010.12.023

Grégoire, L., Anderson, B.A., 2019. Semantic generalization of value-based attentional priority. Learn. Mem. 26, 460-464. https://doi.org/10.1101/lm.050336.119

Haber, S.N., 2011. Neuroanatomy of Reward: A View from the Ventral Striatum, Neurobiology of Sensation and Reward. CRC Press/Taylor \& Francis.

Halverson, H.M., 1927. The Upper Limit of Auditory Localization. Am. J. Psychol. 38, 97. https://doi.org/10.2307/1414648

Hammerschmidt, W., Kagan, I., Kulke, L., Schacht, A., 2018. Implicit reward associations impact face processing: Time-resolved evidence from event-related brain potentials and pupil dilations. Neuroimage 179, 557-569. https://doi.org/10.1016/j.neuroimage.2018.06.055

Henschke, J.U., Dylda, E., Katsanevaki, D., Dupuy, N., Currie, S.P., Amvrosiadis, T., Pakan, J.M.P., Rochefort, N.L., 2020. Reward Association Enhances Stimulus-Specific Representations in Primary Visual Cortex. Curr. Biol. 30, 1866-1880.e5. https://doi.org/10.1016/j.cub.2020.03.018

Herd, S., Krueger, K., Nair, A., Mollick, J., O'Reilly, R., 2021. Neural Mechanisms of Human Decision-Making. Cogn. Affect. Behav. Neurosci. 21, 35-57. https://doi.org/10.3758/s13415020-00842-0

Hickey, C., Chelazzi, L., Theeuwes, J., 2010a. Reward guides vision when it's your thing: Trait reward-seeking in reward-mediated visual priming. PLoS One 5, 14087. https://doi.org/10.1371/journal.pone.0014087

Hickey, C., Chelazzi, L., Theeuwes, J., 2010b. Reward changes salience in human vision via the anterior cingulate. J. Neurosci. 30, 11096-11103. https://doi.org/10.1523/JNEUROSCI.102610.2010

Hickey, C., Di Lollo, V., McDonald, J.J., 2009. Electrophysiological indices of target and distractor processing in visual search. J. Cogn. Neurosci. 21, 760-775. https://doi.org/10.1162/jocn.2009.21039

Hickey, C., Van Zoest, W., 2012. Reward creates oculomotor salience. Curr. Biol. https://doi.org/10.1016/j.cub.2012.02.007

Hoffman, J.E., Nelson, B., 1981. Spatial selectivity in visual search. Percept. Psychophys. 1981303 30, 283-290. https://doi.org/10.3758/BF03214284

Hollerman, J.R., Schultz, W., 1998. Dopamine neurons report an error in the temporal prediction of reward during learning. Nat. Neurosci. 1, 304-309. https://doi.org/10.1038/1124

Hopf, J.M., Boehler, C.N., Luck, S.J., Tsotsos, J.K., Heinze, H.J., Schoenfeld, M.A., 2006. Direct neurophysiological evidence for spatial suppression surrounding the focus of attention in vision. Proc. Natl. Acad. Sci. U. S. A. 103, 1053-1058. https://doi.org/10.1073/pnas.0507746103

Hughes, G., Mathan, S., Yeung, N., 2013. EEG indices of reward motivation and target detectability in a rapid visual detection task. Neuroimage 64, 590-600. https://doi.org/10.1016/j.neuroimage.2012.09.003

Hull, C., 1943. Principles of behavior: An introduction to behavior theory. Appleton-Century-Crofts, New York.

Itthipuripat, S., Vo, V.A., Sprague, T.C., Serences, J.T., 2019. Value-driven attentional capture enhances distractor representations in early visual cortex. PLoS Biol. 17, e3000186. https://doi.org/10.1371/journal.pbio.3000186

Itti, L., Koch, C., 2001. Computational modelling of visual attention. Nat. Rev. Neurosci. 2, 194203. https://doi.org/10.1038/35058500 
Jay, T.M., 2003. Dopamine: A potential substrate for synaptic plasticity and memory mechanisms. Prog. Neurobiol. https://doi.org/10.1016/S0301-0082(03)00085-6

Kahneman, D., Treisman, A., Gibbs, B.J., 1992. The reviewing of object files: Object-specific integration of information. Cogn. Psychol. 24, 175-219. https://doi.org/10.1016/00100285(92)90007-O

Kahneman, D., Tversky, A., 2013. Choices, Values, and Frames. pp. 269-278. https://doi.org/10.1142/9789814417358_0016

Kang, G., Chang, W., Wang, L., Wei, P., Zhou, X., 2018. Reward enhances cross-modal conflict control in object categorization: Electrophysiological evidence. Psychophysiology 55, e13214. https://doi.org/10.1111/psyp.13214

Kang, G., Wang, L., Zhou, X., 2017. Reward interacts with modality shift to reduce cross-modal conflict. J. Vis. 17. https://doi.org/10.1167/17.1.19

Keitel, C., Maess, B., Schröger, E., Müller, M.M., 2013. Early visual and auditory processing rely on modality-specific attentional resources. Neuroimage 70, 240-249. https://doi.org/10.1016/j.neuroimage.2012.12.046

Kim, A.J., Lee, D.S., Anderson, B.A., 2021. Previously reward-associated sounds interfere with goaldirected auditory processing. Q. J. Exp. Psychol. 74, 1257-1263. https://doi.org/10.1177/1747021821990033

Kiss, M., Driver, J., Eimer, M., 2009. Reward Priority of Visual Target Singletons Modulates EventRelated Potential Signatures of Attentional Selection. Psychol. Sci. 20, 245-251. https://doi.org/10.1111/j.1467-9280.2009.02281.x

Klingel, M., Kopčo, N., Laback, B., 2021. Reweighting of Binaural Localization Cues Induced by Lateralization Training. JARO - J. Assoc. Res. Otolaryngol. 1-16. https://doi.org/10.1007/s10162-021-00800-8

Krebs, R.M., Boehler, C.N., Appelbaum, L.G., Woldorff, M.G., 2013. Reward Associations Reduce Behavioral Interference by Changing the Temporal Dynamics of Conflict Processing. PLoS One 8. https://doi.org/10.1371/journal.pone.0053894

Krebs, R.M., Boehler, C.N., Egner, T., Woldorff, M.G., 2011. The neural underpinnings of how reward associations can both guide and misguide attention. J. Neurosci. 31, 9752-9759. https://doi.org/10.1523/JNEUROSCI.0732-11.2011

Krebs, R.M., Boehler, C.N., Woldorff, M.G., 2010. The influence of reward associations on conflict processing in the Stroop task. Cognition 117, 341-347. https://doi.org/10.1016/j.cognition.2010.08.018

Kristjánsson, Á., Sigurjónsdóttir, Ó., Driver, J., 2010. Fortune and reversals of fortune in visual search: Reward contingencies for pop-out targets affect search efficiency and target repetition effects. Attention, Perception, Psychophys. 72, 1229-1236. https://doi.org/10.3758/APP.72.5.1229

Làdavas, E., Tosatto, L., Bertini, C., 2020. Behavioural and functional changes in neglect after multisensory stimulation. Neuropsychol. Rehabil. https://doi.org/10.1080/09602011.2020.1786411

Lakens, D., 2013. Calculating and reporting effect sizes to facilitate cumulative science: a practical primer for t-tests and ANOVAs. Front. Psychol. 4, 863. https://doi.org/10.3389/fpsyg.2013.00863

Le Pelley, M.E., Mitchell, C.J., Beesley, T., George, D.N., Wills, A.J., 2016. Attention and associative learning in humans: An integrative review. Psychol. Bull. 142, 1111-1140. https://doi.org/10.1037/bul0000064

Leo, F., Noppeney, U., 2014. Conditioned Sounds Enhance Visual Processing. PLoS One 9, e106860. https://doi.org/10.1371/journal.pone.0106860

Leo, F., Romei, V., Freeman, E., Ladavas, E., Driver, J., 2011. Looming sounds enhance orientation sensitivity for visual stimuli on the same side as such sounds. Exp. Brain Res. 213, 193-201. https://doi.org/10.1007/s00221-011-2742-8

Liu, D., Deng, J., Zhang, Z., Zhang, Z.Y., Sun, Y.G., Yang, T., Yao, H., 2020. Orbitofrontal control of visual cortex gain promotes visual associative learning. Nat. Commun. 11, 1-14. https://doi.org/10.1038/s41467-020-16609-7

Liu, J., Zhang, C., Zhu, Y., Liu, Y., Sun, H., Ristaniemi, T., Cong, F., Parviainen, T., 2020. Dissociable Effects of Reward on P300 and EEG Spectra Under Conditions of High vs. Low 
Vigilance During a Selective Visual Attention Task. Front. Hum. Neurosci. 14, 207. https://doi.org/10.3389/fnhum.2020.00207

Lockhofen, D.E.L., Mulert, C., 2021. Neurochemistry of Visual Attention. Front. Neurosci. 15, 517. https://doi.org/10.3389/fnins.2021.643597

Luck, S.J., Hillyard, S.A., Mouloua, M., Woldorff, M.G., et al, 1994. Effects of spatial cuing on luminance detectability: Psychophysical and electrophysiological evidence for early selection. J. Exp. Psychol. Hum. Percept. Perform. 20, 887-904. https://doi.org/10.1037//00961523.20.4.887

Luque, D., Beesley, T., Morris, R.W., Jack, B.N., Griffiths, O., Whitford, T.J., Le Pelley, M.E., 2017. Goal-directed and habit-like modulations of stimulus processing during reinforcement learning. J. Neurosci. 37, 3009-3017. https://doi.org/10.1523/JNEUROSCI.3205-16.2017

Luque, D., Morís, J., Rushby, J.A., Le Pelley, M.E., 2015. Goal-directed EEG activity evoked by discriminative stimuli in reinforcement learning. Psychophysiology 52, 238-248. https://doi.org/10.1111/psyp.12302

Maclean, M.H., Giesbrecht, B., 2015. Neural evidence reveals the rapid effects of reward history on selective attention. Brain Res. 1606, 86-94. https://doi.org/10.1016/j.brainres.2015.02.016

Macmillan, N.A., Creelman, C.D., 1991. Detection theory: A user's guide., Detection theory: A user's guide. Cambridge University Press, New York, NY, US.

Maier, M., Ballester, B.R., Verschure, P.F.M.J., 2019. Principles of Neurorehabilitation After Stroke Based on Motor Learning and Brain Plasticity Mechanisms. Front. Syst. Neurosci. https://doi.org/10.3389/fnsys.2019.00074

Mangun, G.R., 1995. Neural mechanisms of visual selective attention. Psychophysiology 32, 4-18. https://doi.org/10.1111/j.1469-8986.1995.tb03400.x

Manohar, S.G., Chong, T.T.J., Apps, M.A.J., Batla, A., Stamelou, M., Jarman, P.R., Bhatia, K.P., Husain, M., 2015. Reward Pays the Cost of Noise Reduction in Motor and Cognitive Control. Curr. Biol. 25, 1707-1716. https://doi.org/10.1016/j.cub.2015.05.038

Manohar, S.G., Muhammed, K., Fallon, S.J., Husain, M., 2019. Motivation dynamically increases noise resistance by internal feedback during movement. Neuropsychologia 123, 19-29. https://doi.org/10.1016/j.neuropsychologia.2018.07.011

Maunsell, J.H.R., 2004. Neuronal representations of cognitive state: Reward or attention? Trends Cogn. Sci. 8, 261-265. https://doi.org/10.1016/j.tics.2004.04.003

McDonald, J.J., Störmer, V.S., Martinez, A., Feng, W., Hillyard, S.A., 2013. Salient Sounds Activate Human Visual Cortex Automatically. J. Neurosci. 33, 9194.

Meredith, M.A., Stein, B.E., 1986. Visual, auditory, and somatosensory convergence on cells in superior colliculus results in multisensory integration. J. Neurophysiol. 56, 640-662. https://doi.org/10.1152/jn.1986.56.3.640

Mine, C., Saiki, J., 2015. Task-irrelevant stimulus-reward association induces value-driven attentional capture. Attention, Perception, Psychophys. 77, 1896-1907. https://doi.org/10.3758/s13414-015-0894-5

Mogami, T., Tanaka, K., 2006. Reward association affects neuronal responses to visual stimuli in macaque TE and perirhinal cortices. J. Neurosci. 26, 6761-6770. https://doi.org/10.1523/JNEUROSCI.4924-05.2006

Mognon, A., Jovicich, J., Bruzzone, L., Buiatti, M., 1994. ADJUST: An automatic EEG artifact detector based on the joint use of spatial and temporal features. Psychophysiology. https://doi.org/10.1111/j.1469-8986.2010.01061.x

Molholm, S., Ritter, W., Murray, M.M., Javitt, D.C., Schroeder, C.E., Foxe, J.J., 2002. Multisensory auditory-visual interactions during early sensory processing in humans: A high-density electrical mapping study, in: Cognitive Brain Research. Elsevier, pp. 115-128. https://doi.org/10.1016/S0926-6410(02)00066-6

Motter, B.C., 1993. Focal attention produces spatially selective processing in visual cortical areas V1, V2, and V4 in the presence of competing stimuli. J. Neurophysiol. 70, 909-919. https://doi.org/10.1152/jn.1993.70.3.909

Mounts, J.R.W., 2000. Attentional capture by abrupt onsets and feature singletons produces inhibitory surrounds. Percept. Psychophys. 62, 1485-1493. https://doi.org/10.3758/BF03212148

Nakayama, K., 1990. The iconic bottleneck and the tenuous link between early visual processing and 
perception, in: Blakemore, C. (Ed.), Vision: Coding and Efficiency. Cambridge: Cambridge University Press, pp. 411-422.

Navarra, J., Alsius, A., Soto-Faraco, S., Spence, C., 2010. Assessing the role of attention in the audiovisual integration of speech. Inf. Fusion 11, 4-11. https://doi.org/10.1016/j.inffus.2009.04.001

Ngo, M.K., Spence, C., 2010. Crossmodal facilitation of masked visual target identification. Attention, Perception, Psychophys. 72, 1938-1947. https://doi.org/10.3758/APP.72.7.1938

Niv, Y., Joel, D., Dayan, P., 2006. A normative perspective on motivation. Trends Cogn. Sci. 10, 375-381. https://doi.org/10.1016/J.TICS.2006.06.010

Nobre, A.C., Rao, A., Chelazzi, L., 2006. Selective attention to specific features within objects: Behavioral and electrophysiological evidence. J. Cogn. Neurosci. 18, 539-561. https://doi.org/10.1162/jocn.2006.18.4.539

Novak, K.D., Foti, D., 2015. Teasing apart the anticipatory and consummatory processing of monetary incentives: An event-related potential study of reward dynamics. Psychophysiology 52, 1470-1482. https://doi.org/10.1111/psyp.12504

O'Doherty, J.P., Cockburn, J., Pauli, W.M., 2017. Learning, Reward, and Decision Making. Annu. Rev. Psychol. 68, 73-100. https://doi.org/10.1146/annurev-psych-010416-044216

Otto, A.R., Daw, N.D., 2019. The opportunity cost of time modulates cognitive effort. Neuropsychologia 123, 92-105. https://doi.org/10.1016/j.neuropsychologia.2018.05.006

Padmala, S., Pessoa, L., 2010. Interactions between cognition and motivation during response inhibition. Neuropsychologia https://doi.org/10.1016/j.neuropsychologia.2009.10.017

Pavlov, 1927. Conditioned Reflexes. Oxford University Press, Oxford.

Pearson, D., Watson, P., Cheng, P.X., Le Pelley, M.E., 2020. Overt Attentional Capture by RewardRelated Stimuli Overcomes Inhibitory Suppression. J. Exp. Psychol. Hum. Percept. Perform. https://doi.org/10.1037/xhp0000728

Pessiglione, M., Schmidt, L., Draganski, B., Kalisch, R., Lau, H., Dolan, R.J., Frith, C.D., 2007. How the Brain Translates Money into Force: A Neuroimaging Study of Subliminal Motivation. Science 316, 904. https://doi.org/10.1126/SCIENCE.1140459

Pessiglione, M., Seymour, B., Flandin, G., Dolan, R.J., Frith, C.D., 2006. Dopamine-dependent prediction errors underpin reward-seeking behaviour in humans. Nature 442, 1042-1045. https://doi.org/10.1038/nature05051

Pessiglione, M., Vinckier, F., Bouret, S., Daunizeau, J., Le Bouc, R., 2018. Why not try harder? Computational approach to motivation deficits in neuro-psychiatric diseases, Brain.

Pessoa, L., 2015. Multiple influences of reward on perception and attention. Vis. cogn. 23, 272-290. https://doi.org/10.1080/13506285.2014.974729

Pessoa, L., 2009. How do emotion and motivation direct executive control? Trends Cogn. Sci. 13, 160-166. https://doi.org/10.1016/j.tics.2009.01.006

Pessoa, L., Engelmann, J.B., 2010. Embedding reward signals into perception and cognition. Front. Neurosci. 4, 17. https://doi.org/10.3389/fnins.2010.00017

Peters, J., Büchel, C., 2010. Neural representations of subjective reward value. Behav. Brain Res. 213, 135-141. https://doi.org/10.1016/J.BBR.2010.04.031

Pfabigan, D.M., Seidel, E.M., Sladky, R., Hahn, A., Paul, K., Grahl, A., Küblböck, M., Kraus, C., Hummer, A., Kranz, G.S., Windischberger, C., Lanzenberger, R., Lamm, C., 2014. P300 amplitude variation is related to ventral striatum BOLD response during gain and loss anticipation: An EEG and fMRI experiment. Neuroimage 96, 12-21. https://doi.org/10.1016/j.neuroimage.2014.03.077

Pick, H.L., Warren, D.H., Hay, J.C., 1969. Sensory conflict in judgments of spatial direction. Percept. Psychophys. 6, 203-205. https://doi.org/10.3758/BF03207017

Platt, M.L., Glimcher, P.W., 1999. Neural correlates of decision variables in parietal cortex. Nature 400, 233-238. https://doi.org/10.1038/22268

Pleger, B., Blankenburg, F., Ruff, C.C., Driver, J., Dolan, R.J., 2008. Reward facilitates tactile judgments and modulates hemodynamic responses in human primary somatosensory cortex. J. Neurosci. 28, 8161-8168. https://doi.org/10.1523/JNEUROSCI.1093-08.2008

Pool, E., Brosch, T., Delplanque, S., Sander, D., 2014. Where is the chocolate? Rapid spatial orienting toward stimuli associated with primary rewards. Cognition 130, 348-359. 
https://doi.org/10.1016/j.cognition.2013.12.002

Pooresmaeili, A., FitzGerald, T.H.B., Bach, D.R., Toelch, U., Ostendorf, F., Dolan, R.J., 2014. Cross-modal effects of value on perceptual acuity and stimulus encoding. Proc. Natl. Acad. Sci. U. S. A. 111, 15244-15249. https://doi.org/10.1073/pnas.1408873111

Pooresmaeili, A., Roelfsema, P.R., 2014. A growth-cone model for the spread of object-based attention during contour grouping. Curr. Biol. 24. https://doi.org/10.1016/j.cub.2014.10.007

Pornpattananangkul, N., Nusslock, R., 2015. Motivated to win: Relationship between anticipatory and outcome reward-related neural activity. Brain Cogn. 100, 21-40. https://doi.org/10.1016/j.bandc.2015.09.002

Posner, M.I., 1980. Orienting of Attention. Q. J. Exp. Psychol. 32, 3-25. https://doi.org/10.1080/00335558008248231

Qin, N., Gu, R., Xue, J., Chen, C., Zhang, M., 2021. Reward-driven attention alters perceived salience. J. Vis. 21, 1-13. https://doi.org/10.1167/JOV.21.1.7

Rangel, A., Camerer, C., Montague, P.R., 2008. A framework for studying the neurobiology of valuebased decision making. Nat. Rev. Neurosci. https://doi.org/10.1038/nrn2357

Raymond, J.E., O’Brien, J.L., 2009. Selective visual attention and motivation: the consequences of value learning in an attentional blink task. Psychol. Sci. 20, 981-8. https://doi.org/10.1111/j.1467-9280.2009.02391.x

Reynolds, J.H., Chelazzi, L., 2004. Attentional modulation of visual processing. Annu. Rev. Neurosci. https://doi.org/10.1146/annurev.neuro.26.041002.131039

Reynolds, J.H., Chelazzi, L., Desimone, R., 1999. Competitive mechanisms subserve attention in macaque areas V2 and V4. J. Neurosci. 19, 1736-1753. https://doi.org/10.1523/jneurosci.1905-01736.1999

Rock, I., Gutman, D., 1981. The effect of inattention on form perception. J. Exp. Psychol. Hum. Percept. Perform. 7, 275-285. https://doi.org/10.1037/0096-1523.7.2.275

Rolls, E.T., 2015. Taste, olfactory, and food reward value processing in the brain. Prog. Neurobiol. https://doi.org/10.1016/j.pneurobio.2015.03.002

Rossi, V., Vanlessen, N., Bayer, M., Grass, A., Pourtois, G., Schacht, A., 2017. Motivational salience modulates early visual cortex responses across task sets. J. Cogn. Neurosci. 29, 968-979. https://doi.org/10.1162/jocn_a_01093

Rusz, D., Bijleveld, E., Kompier, M.A.J., 2018. Reward-associated distractors can harm cognitive performance. PLoS One 13. https://doi.org/10.1371/journal.pone.0205091

Rusz, D., Le Pelley, M.E., Kompier, M.A.J., Mait, L., Bijleveld, E., 2020. Reward-Driven distraction: A meta-analysis. Psychol. Bull. 146, 872-899.

Rutherford, H.J.V., O'Brien, J.L., Raymond, J.E., 2010. Value associations of irrelevant stimuli modify rapid visual orienting. Psychon. Bull. Rev. 17, 536-542. https://doi.org/10.3758/PBR.17.4.536

Rutkowski, R.G., Weinberger, N.M., 2005. Encoding of learned importance of sound by magnitude of representational area in primary auditory cortex. Proc. Natl. Acad. Sci. U. S. A. 102, 1366413669. https://doi.org/10.1073/pnas.0506838102

San Martín, R., Appelbaum, L.G., Huettel, S.A., Woldorff, M.G., 2016. Cortical Brain Activity Reflecting Attentional Biasing Toward Reward-Predicting Cues Covaries with Economic Decision-Making Performance. Cereb. Cortex 26, 1-11. https://doi.org/10.1093/cercor/bhu160

Sanz, L.R.D., Vuilleumier, P., Bourgeois, A., 2018. Cross-modal integration during value-driven attentional capture. $\quad$ Neuropsychologia $120, \quad 105-112$. https://doi.org/10.1016/j.neuropsychologia.2018.10.014

Sawaki, R., Luck, S.J., Raymond, J.E., 2015. How attention changes in response to incentives. J. Cogn. Neurosci. 27, 2229-2239. https://doi.org/10.1162/jocn_a_00847

Schacht, A., Adler, N., Chen, P., Guo, T., Sommer, W., 2012. Association with positive outcome induces early effects in event-related brain potentials. Biol. Psychol. 89, 130-136. https://doi.org/10.1016/j.biopsycho.2011.10.001

Schultz, W., 2015. Neuronal reward and decision signals: From theories to data. Physiol. Rev. 95, 853-951. https://doi.org/10.1152/physrev.00023.2014

Schultz, W., 2013. Updating dopamine reward signals. Curr. Opin. Neurobiol. 23, 229-238. https://doi.org/10.1016/J.CONB.2012.11.012

Schultz, W., 2006. Behavioral Theories and the Neurophysiology of Reward. Annu. Rev. Psychol. 
57, 87-115. https://doi.org/10.1146/annurev.psych.56.091103.070229

Schultz, W., 2002. Getting Formal with Dopamine and Reward. Neuron 36, 241-263. https://doi.org/10.1016/S0896-6273(02)00967-4

Schultz, W., 2000. Multiple reward signals in the brain 1, 199-207. https://doi.org/10.1038/35044563

Schultz, W., 1998. Predictive Reward Signal of Dopamine Neurons. J. Neurophysiol. 80, 1-27. https://doi.org/10.1152/jn.1998.80.1.1

Schultz, W., 1986. Responses of midbrain dopamine neurons to behavioral trigger stimuli in the monkey. J. Neurophysiol. 56, 1439-1461. https://doi.org/10.1152/jn.1986.56.5.1439

Schultz, W., Dayan, P., Montague, P.R., 1997. A Neural Substrate of Prediction and Reward. Science (80-. ). 275, 1593-1599. https://doi.org/10.1126/science.275.5306.1593

Seitz, A.R., Kim, R., Shams, L., 2006. Sound Facilitates Visual Learning. Curr. Biol. 16, 1422-1427. https://doi.org/10.1016/j.cub.2006.05.048

Serences, J.T., 2008. Value-Based Modulations in Human Visual Cortex. Neuron 60, 1169-1181. https://doi.org/10.1016/j.neuron.2008.10.051

Serences, J.T., Saproo, S., 2010. Population response profiles in early visual cortex are biased in favor of more valuable stimuli. J. Neurophysiol. 104, 76-87. https://doi.org/10.1152/jn.01090.2009

Serences, J.T., Saproo, S., Scolari, M., Ho, T., Muftuler, L.T., 2009. Estimating the influence of attention on population codes in human visual cortex using voxel-based tuning functions. Neuroimage 44, 223-231. https://doi.org/10.1016/J.NEUROIMAGE.2008.07.043

Shadmehr, R., Xivry, J.J.O. de, Xu-Wilson, M., Shih, T.-Y., 2010. Temporal Discounting of Reward and the Cost of Time in Motor Control. J. Neurosci. 30, 10507-10516. https://doi.org/10.1523/JNEUROSCI.1343-10.2010

Shams, L., Kamitani, Y., Shimojo, S., 2000. Illusions: What you see is what you hear. Nature 408, 788. https://doi.org/10.1038/35048669

Shams, L., Seitz, A.R., 2008. Benefits of multisensory learning. Trends Cogn. Sci. 12, 411-417. https://doi.org/10.1016/j.tics.2008.07.006

Shapcott, K.A., Schmiedt, J.T., Kouroupaki, K., Kienitz, R., Lazar, A., Singer, W., Schmid, M.C., 2020. Reward-related suppression of neural activity in macaque visual area v4. Cereb. Cortex 30, 4871-4881. https://doi.org/10.1093/cercor/bhaa079

Shuler, M.G., Bear, M.F., 2006. Reward timing in the primary visual cortex. Science (80-. ). 311, 1606-1609. https://doi.org/10.1126/science.1123513

Slutsky, D.A., Recanzone, G.H., 2001. Temporal and spatial dependency of the ventriloquism effect. Neuroreport 12, 7-10. https://doi.org/10.1097/00001756-200101220-00009

Sobal, J., 1998. Cultural Comparison Research Designs in Food, Eating, and Nutrition. Food Qual. Prefer. 9, 385-392. https://doi.org/10.1016/S0950-3293(98)00029-9

Stanisor, L., Van Der Togt, C., Pennartz, C.M.A., Roelfsema, P.R., 2013. A unified selection signal for attention and reward in primary visual cortex. Proc. Natl. Acad. Sci. U. S. A. 110, $9136-$ 9141. https://doi.org/10.1073/pnas.1300117110

Stein, B.E., Meredith, M.A., 1993. The merging of the senses. The MIT Press.

Steinberg, E.E., Keiflin, R., Boivin, J.R., Witten, I.B., Deisseroth, K., Janak, P.H., 2013. A causal link between prediction errors, dopamine neurons and learning. Nat. Neurosci. 16, 966-973. https://doi.org/10.1038/nn.3413

Stine, G.M., Zylberberg, A., Ditterich, J., Shadlen, M.N., 2020. Differentiating between integration and non-integration strategies in perceptual decision making. Elife 9. https://doi.org/10.7554/eLife.55365

Störmer, V.S., Alvarez, G.A., 2014. Feature-based attention elicits surround suppression in feature space. Curr. Biol. 24, 1985-1988. https://doi.org/10.1016/j.cub.2014.07.030

Störmer, V.S., McDonald, J.J., Hillyard, S.A., 2009. Cross-modal cueing of attention alters appearance and early cortical processing of visual stimuli. Proc. Natl. Acad. Sci. U. S. A. 106, 22456-22461. https://doi.org/10.1073/pnas.0907573106

Sutton, R.S., Barto, A.G., 2018. Reinforcement Learning: An Introduction. MIT Press, Cambridge; MA.

Takakuwa, N., Kato, R., Redgrave, P., Isa, T., 2017. Emergence of visually-evoked reward expectation signals in dopamine neurons via the superior colliculus in V1 lesioned monkeys. 
Elife 6. https://doi.org/10.7554/eLife.24459

Takikawa, Y., Kawagoe, R., Itoh, H., Nakahara, H., Hikosaka, O., 2002. Modulation of saccadic eye movements by predicted reward outcome. Exp. Brain Res. 142. https://doi.org/10.1007/s00221001-0928-1

Talsma, D., Doty, T.J., Woldorff, M.G., 2007. Selective attention and audiovisual integration: Is attending to both modalities a prerequisite for early integration? Cereb. Cortex 17, 679-690. https://doi.org/10.1093/cercor/bhk016

Talsma, D., Woldorff, M.G., 2005. Selective attention and multisensory integration: Multiple phases of effects on the evoked brain activity. J. Cogn. Neurosci. 17, 1098-1114. https://doi.org/10.1162/0898929054475172

Tankelevitch, L., Spaak, E., Rushworth, M., Stokes, M., 2019. Previously reward-associated stimuli capture spatial attention in the absence of changes in the corresponding sensory representations as measured with MEG. bioRxiv 622589. https://doi.org/10.1101/622589

Tankelevitch, L., Spaak, E., Rushworth, M.F.S., Stokes, M.G., 2020. Previously Reward-Associated Stimuli Capture Spatial Attention in the Absence of Changes in the Corresponding Sensory Representations as Measured with MEG. J. Neurosci. 40, 5033-5050. https://doi.org/10.1523/JNEUROSCI.1172-19.2020

Teder-Sälejärvi, W.A., Di Russo, F., McDonald, J.J., Hillyard, S.A., 2005. Effects of spatial congruity on audio-visual multimodal integration. J. Cogn. Neurosci. 17, 1396-1409. https://doi.org/10.1162/0898929054985383

Teder-Sälejärvi, W.A., McDonald, J.J., Di Russo, F., Hillyard, S.A., 2002. An analysis of audiovisual crossmodal integration by means of event-related potential (ERP) recordings, in: Cognitive Brain Research. https://doi.org/10.1016/S0926-6410(02)00065-4

Theeuwes, J., 2019. Goal-driven, stimulus-driven, and history-driven selection. Curr. Opin. Psychol. https://doi.org/10.1016/j.copsyc.2018.12.024

Theeuwes, J., 2018. Visual selection: Usually fast and automatic; Seldom slow and volitional. J. Cogn. 1. https://doi.org/10.5334/joc.13

Theeuwes, J., Belopolsky, A. V., 2012. Reward grabs the eye: Oculomotor capture by rewarding stimuli. Vision Res. 74, 80-85. https://doi.org/10.1016/j.visres.2012.07.024

Thorndike, E.L., 1911. Animal intelligence. Macmillan, New York.

Trappenberg, T.P., Dorris, M.C., Munoz, D.P., Klein, R.M., 2001. A model of saccade initiation based on the competitive integration of exogenous and endogenous signals in the superior colliculus. J. Cogn. Neurosci. 13, 256-271. https://doi.org/10.1162/089892901564306

Vakhrushev, R., Cheng, F., Schacht, A., Pooresmaeili, A., 2021. Differential effects of intra-modal and cross-modal reward value on visual perception: ERP evidence. bioRxiv 2021.09.29.462374. https://doi.org/10.1101/2021.09.29.462374

Van Den Berg, B., Krebs, R.M., Lorist, M.M., Woldorff, M.G., 2014. Utilization of reward-prospect enhances preparatory attention and reduces stimulus conflict. Cogn. Affect. Behav. Neurosci. 14, 561-577. https://doi.org/10.3758/s13415-014-0281-Z

Van Zoest, W., Donk, M., Theeuwes, J., 2004. The role of stimulus-driven and goal-driven control in saccadic visual selection. J. Exp. Psychol. Hum. Percept. Perform. 30, 746-759. https://doi.org/10.1037/0096-1523.30.4.749

Vitay, J., Hamker, F.H., 2007. On the role of dopamine in cognitive vision, in: Lecture Notes in Computer Science (Including Subseries Lecture Notes in Artificial Intelligence and Lecture Notes in Bioinformatics). Springer Verlag, pp. 352-366. https://doi.org/10.1007/978-3-54077343-6_23

Von Kriegstein, K., Giraud, A.L., 2006. Implicit multisensory associations influence voice recognition. PLoS Biol. 4, 1809-1820. https://doi.org/10.1371/journal.pbio.0040326

Wang, L., Yu, H., Zhou, X., 2013. Interaction between value and perceptual salience in value-driven attentional capture. J. Vis. 13, 5-5. https://doi.org/10.1167/13.3.5

Watson, A.B., Pelli, D.G., 1983. Quest: A Bayesian adaptive psychometric method. Percept. Psychophys. 33, 113-120. https://doi.org/10.3758/BF03202828

Watson, P., Pearson, D., Most, S.B., Theeuwes, J., Wiers, R.W., Le Pelley, M.E., 2019. Attentional capture by Pavlovian reward-signalling distractors in visual search persists when rewards are removed. PLoS One 14, e0226284. https://doi.org/10.1371/journal.pone.0226284

Watson, R., Latinus, M., Noguchi, T., Garrod, O., Crabbe, F., Belin, P., 2014. Crossmodal adaptation 
in right posterior superior temporal sulcus during face-voice emotional integration. J. Neurosci. 34, 6813-6821. https://doi.org/10.1523/JNEUROSCI.4478-13.2014

Weil, R.S., Furl, N., Ruff, C.C., Symmonds, M., Flandin, G., Dolan, R.J., Driver, J., Rees, G., 2010. Rewarding Feedback After Correct Visual Discriminations Has Both General and Specific Influences on Visual Cortex. J. Neurophysiol. 104, 1746-1757. https://doi.org/10.1152/jn.00870.2009

Weinberger, N.M., 2007. Associative representational plasticity in the auditory cortex: A synthesis of two disciplines. Learn. Mem. https://doi.org/10.1101/lm.421807

Welch, R.B., Warren, D.H., 1980. Immediate perceptual response to intersensory discrepancy. Psychol. Bull. 88, 638-667. https://doi.org/10.1037/0033-2909.88.3.638

Westbrook, A., Frank, M.J., Cools, R., 2021. A mosaic of cost-benefit control over cortico-striatal circuitry. Trends Cogn. Sci. https://doi.org/10.1016/j.tics.2021.04.007

Wikman, P., Rinne, T., Petkov, C.I., 2019. Reward cues readily direct monkeys' auditory performance resulting in broad auditory cortex modulation and interaction with sites along cholinergic and dopaminergic pathways. Sci. Rep. 9. https://doi.org/10.1038/s41598-01938833-y

Wild, B., Treue, S., 2021. Primate extrastriate cortical area MST: a gateway between sensation and cognition. J. Neurophysiol. 125, 1851-1882. https://doi.org/10.1152/jn.00384.2020

Wise, R.A., 2004. Dopamine, learning and motivation. Nat. Rev. Neurosci. https://doi.org/10.1038/nrn1406

Wittmann, M., Leland, D.S., Paulus, M.P., 2007. Time and decision making: differential contribution of the posterior insular cortex and the striatum during a delay discounting task. Exp. Brain Res. 20061794 179, 643-653. https://doi.org/10.1007/S00221-006-0822-Y

Woldorff, M.G., 1993. Distortion of ERP averages due to overlap from temporally adjacent ERPs: Analysis and correction. Psychophysiology 30, 98-119. https://doi.org/10.1111/j.14698986.1993.tb03209.x

Woldorff, M.G., Fox, P.T., Matzke, M., Lancaster, J.L., Veeraswamy, S., Zamarripa, F., Seabolt, M., Glass, T., Gao, J.H., Martin, C.C., Jerabek, P., 1997. Retinotopic organization of early visual spatial attention effects as revealed by PET and ERPs, in: Human Brain Mapping. Hum Brain Mapp, pp. 280-286. https://doi.org/10.1002/(SICI)1097-0193(1997)5:4<280::AIDHBM13>3.0.CO;2-I

Wolfe, J.M., 2021. Guided Search 6.0: An updated model of visual search. Psychon. Bull. Rev. 28, 1060-1092. https://doi.org/10.3758/s13423-020-01859-9

Woods, T.M., Recanzone, G.H., 2004. Cross-Modal Interactions Evidenced, in: The Handbook of Multisensory Processes. MIT Press, Cambridge, MA, pp. 35-48.

Yantis, S., Anderson, B.A., Wampler, E.K., Laurent, P.A., 2012. Reward and attentional control in visual search. Nebraska Symp. Motiv. https://doi.org/10.1007/978-1-4614-4794-8-5

Yeung, N., Sanfey, A.G., 2004. Independent coding of reward magnitude and valence in the human brain. J. Neurosci. 24, 6258-6264. https://doi.org/10.1523/JNEUROSCI.4537-03.2004

Zelinsky, G.J., Bisley, J.W., 2015. The what, where, and why of priority maps and their interactions with visual working memory. Ann. N. Y. Acad. Sci. 1339, 154-164. https://doi.org/10.1111/nyas.12606

Zimmer, U., Itthipanyanan, S., Grent-'T-Jong, T., Woldorff, M.G., 2010. The electrophysiological time course of the interaction of stimulus conflict and the multisensory spread of attention. Eur. J. Neurosci. 31, 1744-1754. https://doi.org/10.1111/j.1460-9568.2010.07229.x 


\subsection{Supplementary Information}

\section{ERP results when contralateral posterior ERPs were examined}

We evaluated the amplitude of ERP components (PA, P1, N1, and P3) in contralateral electrodes (Supplementary Figure 4). To this end, responses of O2 and PO8 to the stimuli presented on the left hemi-field and responses of $\mathrm{O} 1$ and PO7 to the stimuli on the right hemi-field were measured. The contralateral ERPs were then averaged across the two sides. Two-way ANOVAs with reward value and modality as independent factors and amplitude of each component as dependent factor revealed only a trend for an interaction between reward value and modality in $\mathrm{N} 1$ window $\left(\mathrm{F}_{(1,35)}=3.19, \mathrm{p}=0.083, \eta_{\mathrm{p}}{ }^{2}=0.083\right)$, corresponding to a decrease in N1 negativity for cross-modal, high- compared to low-value condition (mean \pm s.e.m: $0.41 \pm 0.28$ and $-0.49 \pm 0.39$ for high- and low-value stimuli respectively, $\left.\mathrm{t}(35)=2.3, \mathrm{p}=0.025, \mathrm{~d}_{\mathrm{z}}=0.389\right)$. Furthermore, a main effect of reward value was found in $\mathrm{P} 3$ window $\left(\mathrm{F}_{(1,35)}=9.14, \mathrm{p}=0.005, \eta_{\mathrm{p}}{ }^{2}=0.207\right)$. Planed pairwise comparisons revealed a significant enhancement of contralateral P3 responses for high- compared to lowvalue cross-modal cues (mean \pm s.e.m: $0.5 \pm 0.28$ and $-0.59 \pm 0.44$, for high- compared to low-value cross-modal cues respectively, $\mathrm{t}(35)=2.388, \mathrm{p}=0.022, \mathrm{~d}_{\mathrm{z}}=0.398$ ). 


\section{$\mathrm{P3}$ responses of midline electrodes during conditioning}

Previous studies have reported robust reward modulations of P3 responses of midline electrodes $(\mathrm{Pz}, \mathrm{CPz}, \mathrm{Cz}, \mathrm{FCz}$ and $\mathrm{Fz})$ time-locked to the presentation of reward cues, both for both visual and auditory cues (Gehring and Willoughby, 2002; Glazer et al., 2018; Krugliakova et al., 2019, 2018; Van Den Berg et al., 2014). Based on these and our preregistered plan, we next inspected the ERP responses of the midline electrodes (Supplementary Figure 1), time-locked to the presentation of the reward cues in the P3 window (300-600 ms). The frontocentral electrodes are most sensitive to auditory signals (Fz, FCz), whereas the posterior midline electrodes are most sensitive to visual cues $(\mathrm{Cz}, \mathrm{Pz})$ or both $(\mathrm{Cz})$, based on the studies reported above. Therefore, we expected to find a significant difference in P3 amplitudes for stimuli of different modalities, reflecting the different topography of areas processing visual or auditory information. In addition, we also expected an effect of reward value for one or both modalities across these electrodes. We did not find an overall robust value-driven modulation based on our preregistered analysis plan. Specifically, a rmANOVA revealed a main effect of modality $\left(\mathrm{F}_{(1,35)}=4.64, \mathrm{P}=0.038\right.$, $\left.\eta_{\mathrm{p}}{ }^{2}=0.117\right)$, a main effect of electrode $\left(\mathrm{F}_{(4,140)}=124.73, \mathrm{p}<0.001, \eta_{\mathrm{p}}{ }^{2}=0.781\right)$ and a trend for modality and electrode interaction $\left(\mathrm{F}_{(4,140)}=3.53, \mathrm{p}=0.054, \eta_{\mathrm{p}}{ }^{2}=0.092\right)$, as expected. Other comparisons did not reach significance: main effect of value $\left(F_{(1,35)}=1.6, p=0.214\right)$, interaction between value and modality $\left(\mathrm{F}_{(1,35)}=3.19, \mathrm{p}=0.083\right)$, interaction between value and electrode $\mathrm{F}(1,35)<1, \mathrm{p}=0.381)$ and interaction between factors value, modality and electrode $\left(\mathrm{F}_{(1,35)}=1.34, \mathrm{p}=0.054\right)$. Although the inspection of ERP responses (Supplementary Figure 1) suggests value-driven modulations in some of these electrodes for visual (esp. Pz), auditory (esp. Fz), or both cues types (Cz), in order to adhere to our preregistered plan, we did not further investigate these effects.

\section{ERP results when only correct trials were included}

We examined the ERP responses of the posterior ROI when only correct trials of each condition were included in our analysis (Supplementary Figure 5). To this end, we performed rmANOVAs on the amplitude of PA, P1, N1 and P3 and the latency of P1 and N1 components of the ERPs with reward value (high or low) and modality (intra-modal or cross-modal) as factors. Overall, we obtained the same results as results obtained with all trials, albeit some of our reported were even stronger when only correct trials were included.

In PA (90-120 ms) window we found an interaction effect between factors modality and reward value $\left(\mathrm{F}(1,35)=10.54, \mathrm{p}=0.003, \eta_{\mathrm{p}}{ }^{2}=0.231\right)$. Intra-modal high-value cues 
suppressed PA amplitudes compared to low-value cues (mean \pm s.e.m: $-0.58 \pm 0.35$ and 0.41 \pm 0.27 , for the PA amplitude of high- compared to low-value cues, $t(35)=-2.475, p=0.018$, $\mathrm{d}_{\mathrm{z}}=0.412$ ). Cross-modal high value cues on the other hand increased the PA amplitude compared to low value cues (mean \pm s.e.m: $0.02 \pm 0.2$ and $-0.86 \pm 0.33$, for the PA amplitude of high- compared to low-value cues, $\mathrm{t}(35)=2.278, \mathrm{p}=0.029, \mathrm{~d}_{\mathrm{z}}=0.380$ ).

Analysis of $\mathrm{P} 1$ component revealed only a main effect of modality $\left(\mathrm{F}_{(1,35)}=5.33, \mathrm{P}=\right.$ $0.027, \eta_{\mathrm{p}}{ }^{2}=0.132$ ) but no main or interaction effect with reward value (both ps>0.1).

In N1 (170-250 ms) window, we found an interaction effect between reward value and modality $\left(\mathrm{F}(1,35)=6.46, \mathrm{P}=0.016, \eta_{\mathrm{p}}{ }^{2}=0.156\right)$. Cross-modal high-value cues decreased the N1 negativity compared to low value cues (mean \pm s.e.m: $1.21 \pm 0.37$ and $\left.0.46 \pm 0.41, \mathrm{t}(35)=3.245, \mathrm{p}=0.003, \mathrm{~d}_{\mathrm{z}}=0.541\right)$. The modulation of $\mathrm{N} 1$ amplitude was not significant for intra-modal cues.

In P3 window (300-600 ms), we found no main effect of modality, reward value or an interaction between reward and modality.

The analysis of the latency of $\mathrm{P} 1$ and $\mathrm{N} 1$ components revealed only a significant value $\times$ modality interaction effect for $\mathrm{N} 1$ latency $\left(\mathrm{F}(1,35)=6.38, \mathrm{P}=0.016, \eta_{\mathrm{p}}{ }^{2}=0.154\right)$. Intramodal, high value cues elicited faster $\mathrm{N} 1$ responses compared to low values cues (mean \pm s.e.m: $-4.94 \pm 4.61$ and $8.33 \pm 3.39$, for N1 latency of high- and low-value cues respectively corrected for pre-conditioning differences, $\mathrm{t}(35)=-2.228, \mathrm{p}=0.032, \mathrm{~d}_{\mathrm{z}}=0.371$ ). 

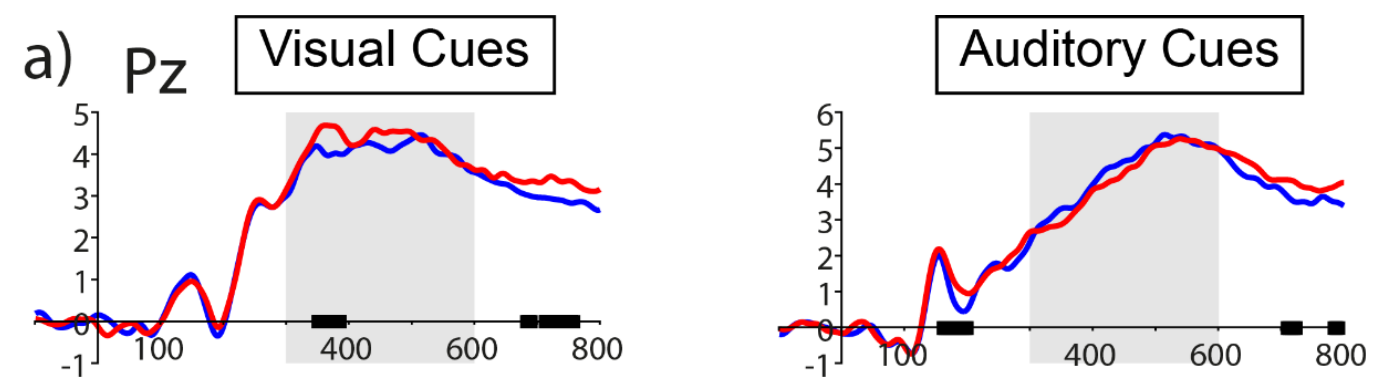

b) $\mathrm{CPz}$
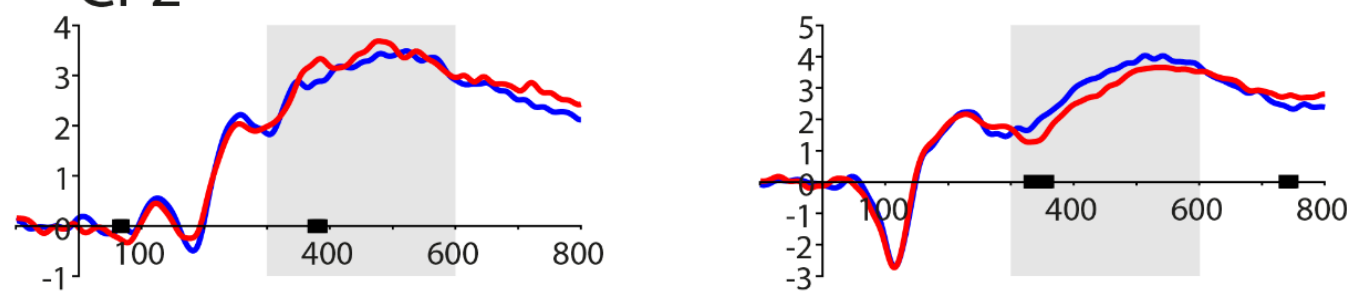

c) $\mathrm{Cz}$
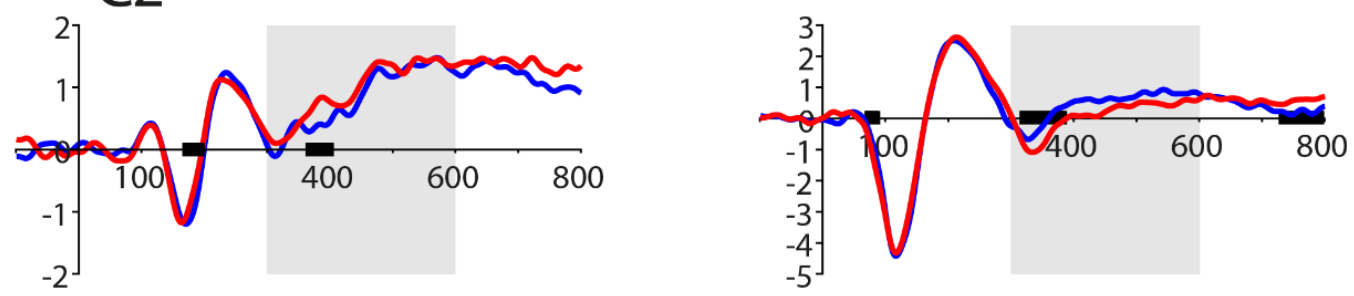

d) $\mathrm{FCz}$
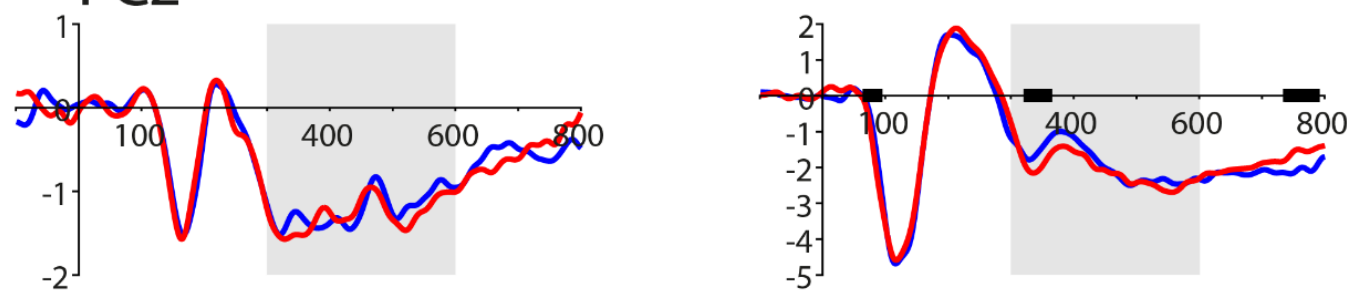

e) $\mathrm{Fz}$

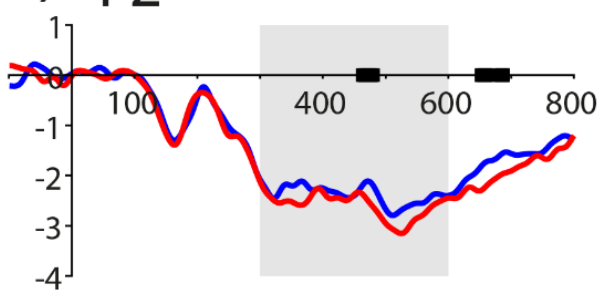

High value

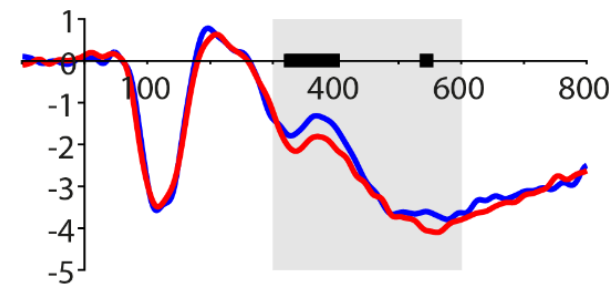

Low value

Supplementary Figure 1. ERPs of midline electrodes during the reward associative learning (conditioning phase). Black marks on $\mathrm{x}$ axis correspond to times points when a significant difference between high and low reward value cues were found (uncorrected $\mathrm{p}<0.05$ ), as measured by a moving window of $10 \mathrm{~ms}$. 
a) $\mathrm{Pz}$ Intra-modal

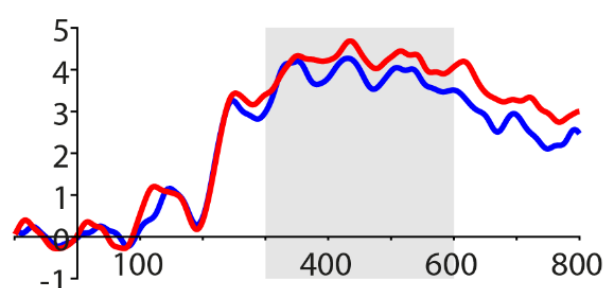

b) $\mathrm{CPz}$

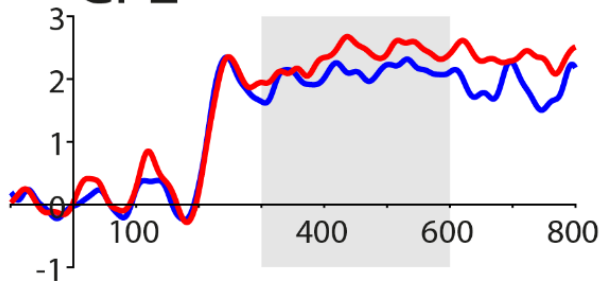

C) $\mathrm{Cz}$

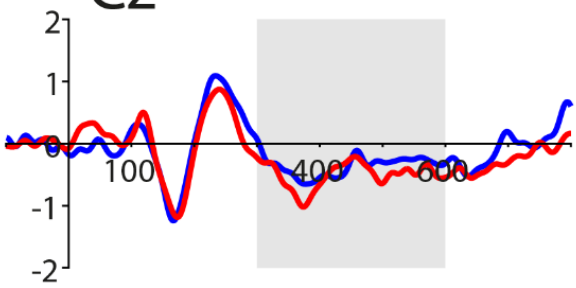

d) $\mathrm{FCz}$

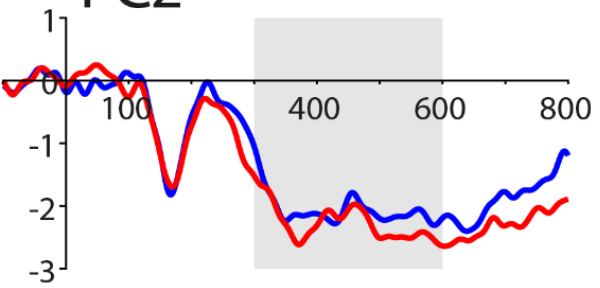

e) $\mathrm{Fz}$

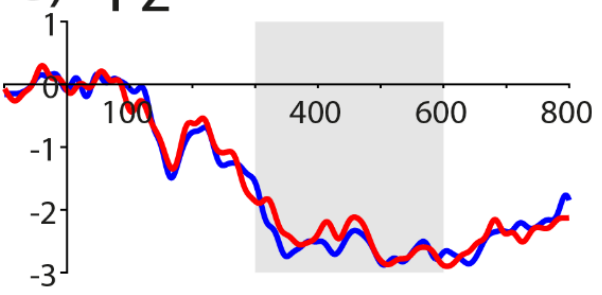

Cross-modal
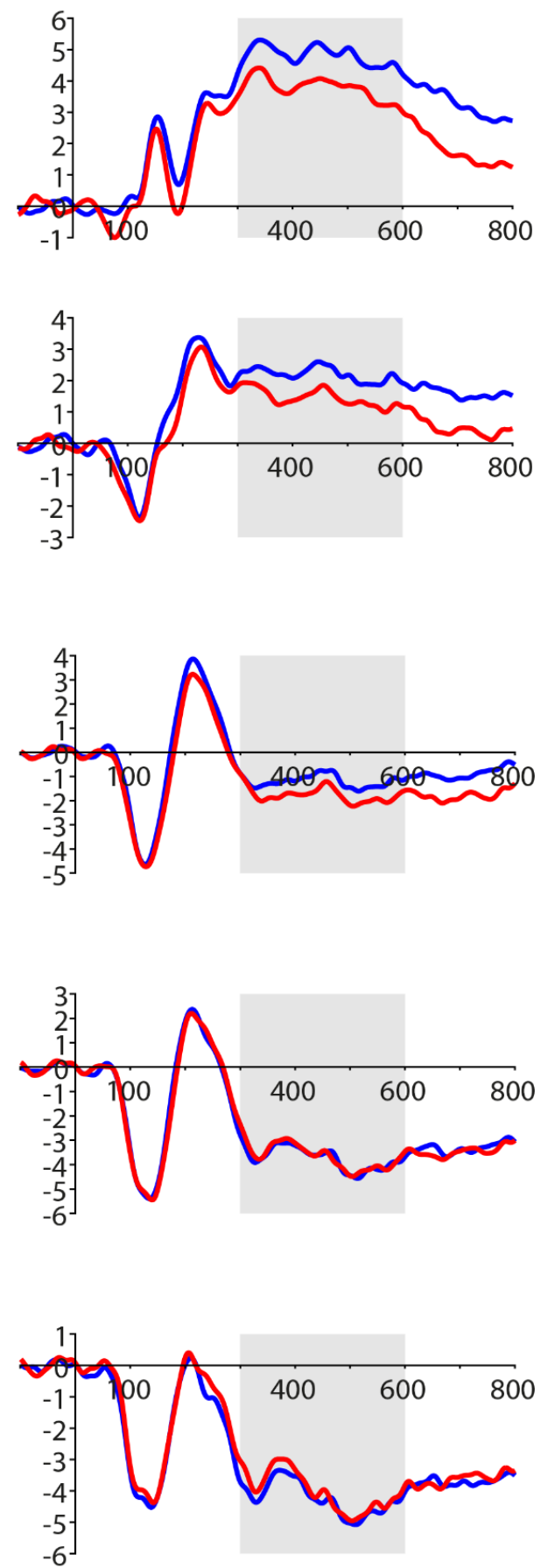

Low value

Supplementary Figure 2. ERPs of midline electrodes during the pre-conditioning phase. 

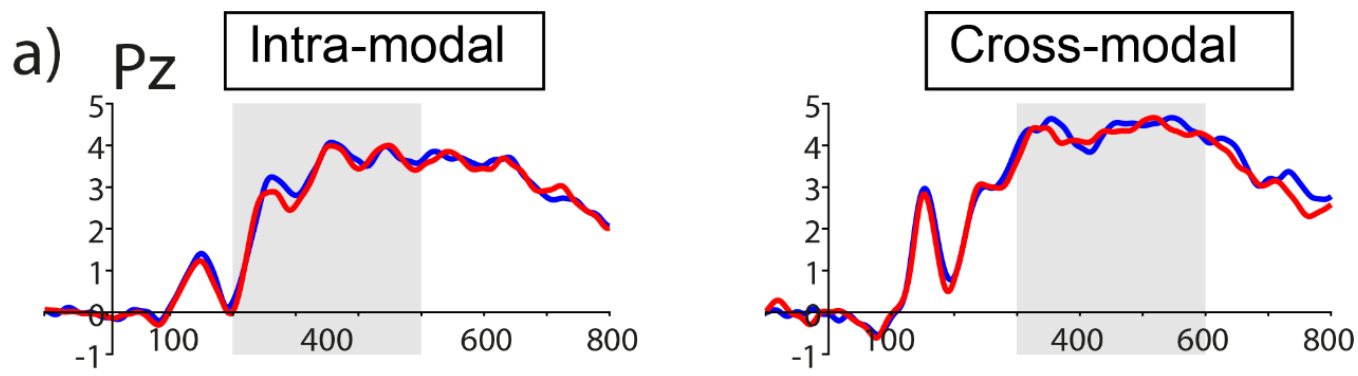

b) $\mathrm{CPz}$
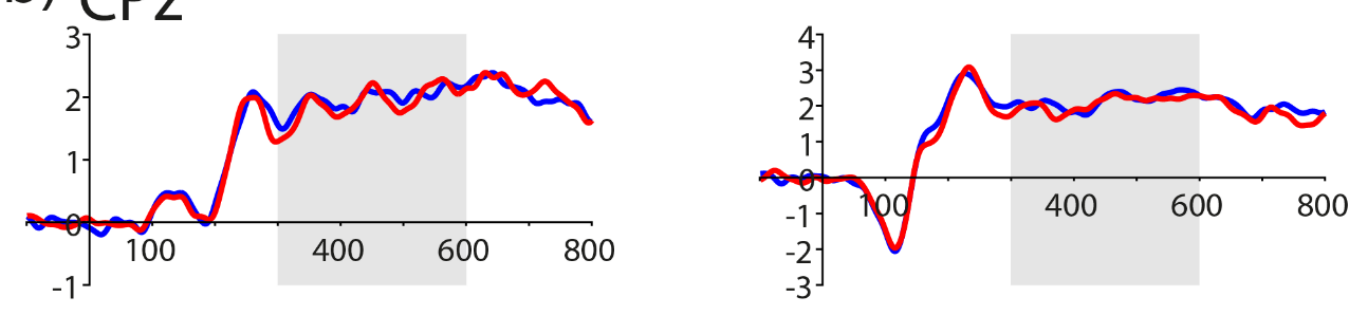

c) $\mathrm{Cz}$
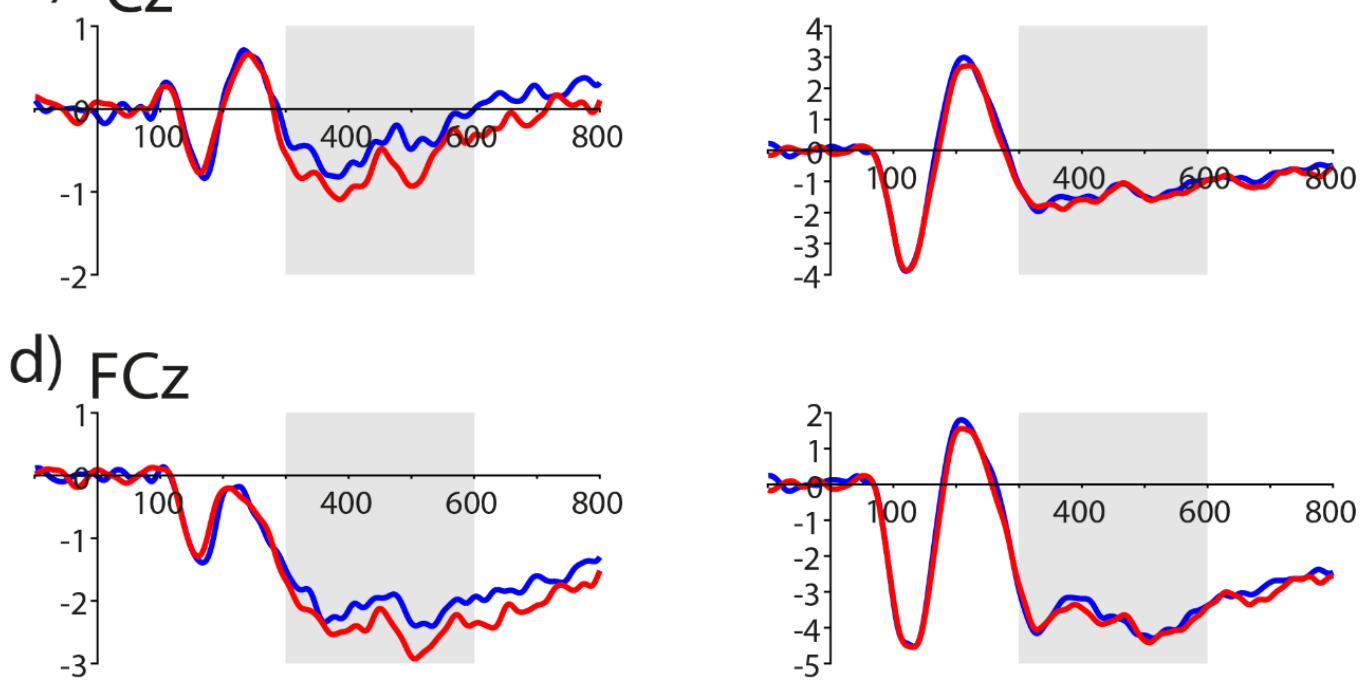

e) $\mathrm{Fz}$
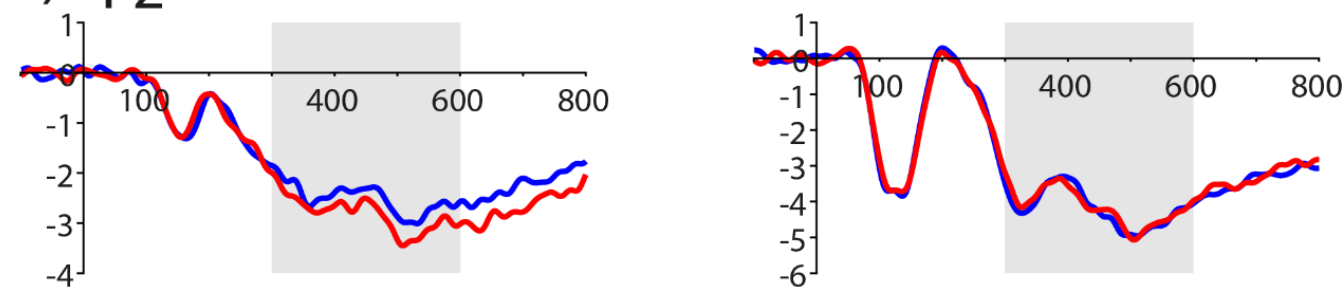

High value

Low value

Supplementary Figure 3. ERPs of midline electrodes during the post-conditioning phase. 

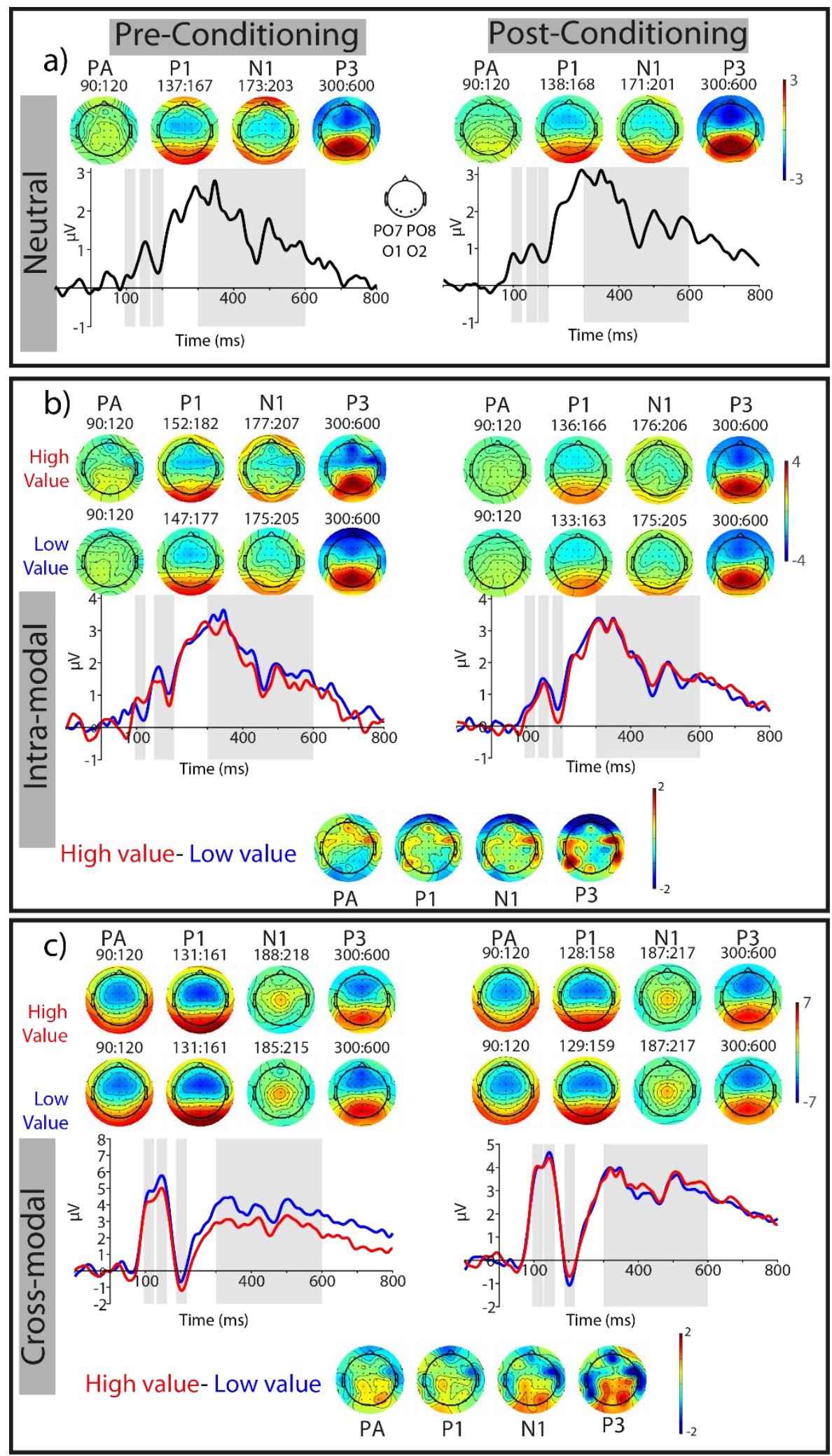

Supplementary Figure 4. Contralateral responses of the posterior ROI during pre- and post-conditioing phases, see also Figure 4 in the main text. 

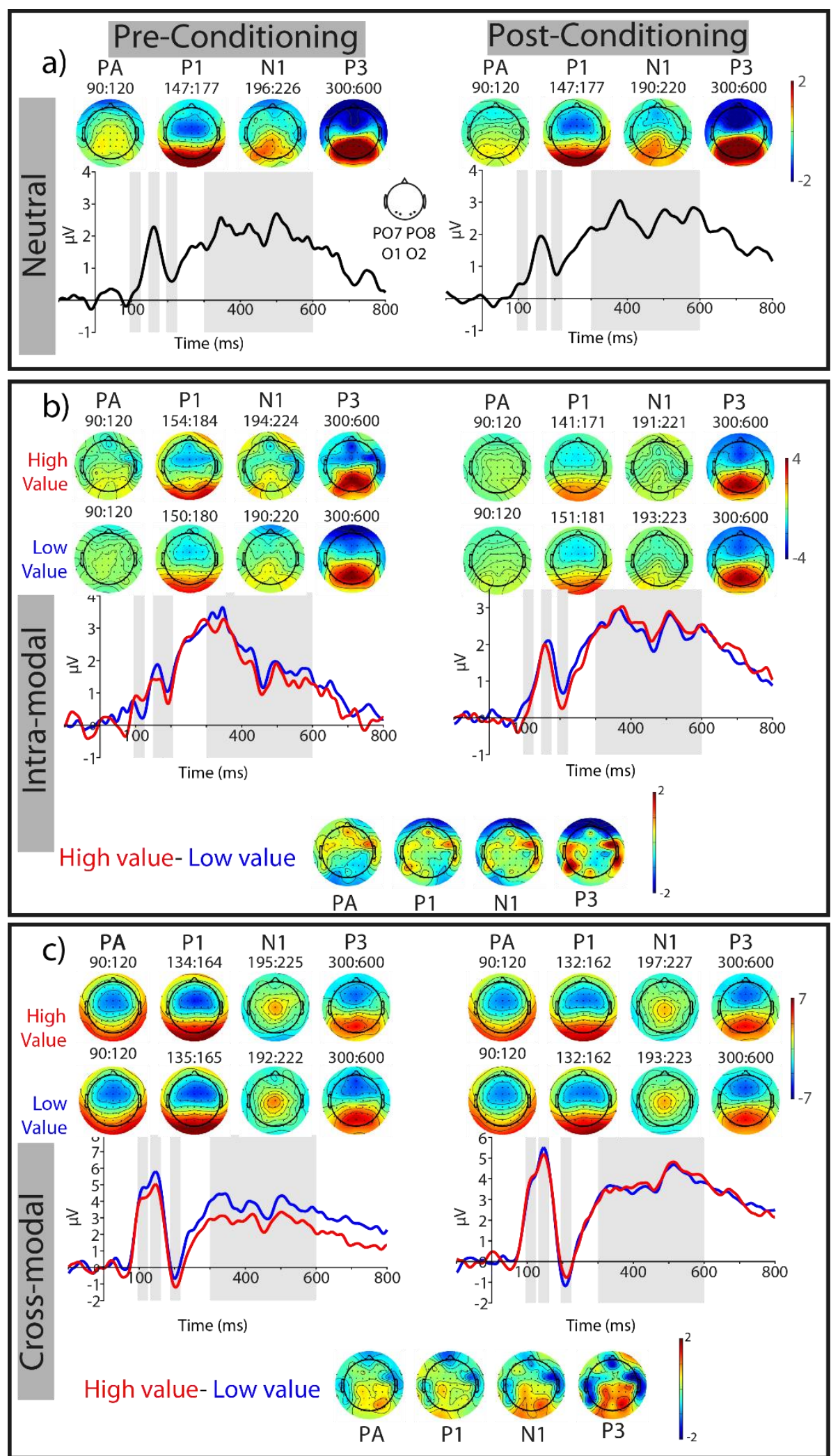

Supplementary Figure 5. ERP results of the posterior ROI when only correct trials were included, see also Figure 4 in the main text. 


\section{Chapter 3: Spatiotemporal characteristics of the capture of attention by reward cues from different sensory modalities}

Roman Vakhrushev*1, Arezoo Pooresmaeili ${ }^{1}$

${ }^{1}$ Perception and Cognition Lab, European Neuroscience Institute Goettingen- A Joint Initiative of the University Medical Center Goettingen and the Max-Planck-Society, Germany, Grisebachstrasse 5, 37077 Goettingen, Germany

* Corresponding author: r.vakhrushev@eni-g.de 


\begin{abstract}
Stimuli that have been previously associated with the delivery of reward capture attention and modulate behavioral and neuronal responses. These effects were shown in visual and auditory modalities as well as across modalities, yet the spatiotemporal characteristics of intra- and cross-modal reward effects were not directly compared. We tested effects of previously rewarded, task-irrelevant visual (intra-modal) and auditory (cross-modal) cues on a visual orientation discrimination task while manipulating the spatial focus of attention (experiment 1) and the temporal asynchrony of the reward cues (Experiment 2). Results of the first experiment revealed a difference between intra- and cross-modal reward effects. When attention was narrowly focused, intra-modal high reward cues captured attention toward the target and sped up response times. Cross-modal high reward cues, on the other hand, enhanced the visual sensitivity only when attentional focus was wide. These results indicate that intra-modal and cross-modal rewards exert their effects through engaging different sizes of attentional focus. The findings of experiment 1 under a wide focus of attention were replicated in experiment 2 , and the strongest effects were observed when reward cues and the target were presented synchronously. These results demonstrate that the associated value of task-irrelevant reward cues promotes their integration with the target, thereby allowing the prioritized processing of reward signals to spread to the target and that this effect operates under different sizes of the attentional focus for intra-modal and crossmodal cues.
\end{abstract}

Keywords: reward, focus of attention, sensory modality. 


\subsection{Introduction}

Reward signals determine animals' behavior (Berridge and Kringelbach, 2008; Schultz, 2015) by biasing them towards signals that deliver rewards. Previous findings showed that cues associated with high reward outcomes affect human behavioral responses by improving response time and accuracy (for review see: Anderson et al., 2021; Pessoa, 2015). These effects were present even when reward cues were irrelevant to the task or when they were measured at the time that reward delivery was halted, i.e. the extinction phase (Anderson et al., 2011a; Failing and Theeuwes, 2018; Hickey et al., 2010b; Pooresmaeili et al., 2014; Tankelevitch et al., 2020). For example, Anderson et al. (2011a) demonstrated that visual task-irrelevant high reward cues captured visual spatial attention and impaired behavioral performance when reward cues appeared as a distractor. The automatic nature of reward effects (Hickey and Van Zoest, 2012; Watson et al., 2019) suggests that reward signals influence sensory processing at the early stages (Rossi et al., 2017; Shuler and Bear, 2006). Indeed, previous findings reported prioritized sensory processing of high reward cues when these cues were delivered intra-modally. For instance, studies reported reward effects on behavioral performance and neuronal representation of the reward cues in visual (Goldstein et al., 2006; Shuler and Bear, 2006; Weil et al., 2010), auditory (Rutkowski and Weinberger, 2005), olfactory (Grabenhorst et al., 2011; Pool et al., 2014), tactile (Pleger et al., 2008), or gustatory (Rolls, 2015) modalities. Moreover, reward cues could influence sensory processing cross-modally (Anderson, 2016a; Cheng et al., 2020; Leo and Noppeney, 2014; Pooresmaeili et al., 2014). Cross-modal reward cues, as well as intra-modal reward cues, exert similar modulatory effects, where reward cues improve behavioral performance and neuronal responses when they could integrate with the target (Baldassi and Simoncini, 2011; Pooresmaeili et al., 2014) and impair behavioral performance when appear as a distractor (Anderson, 2016a; Kim et al., 2021). Thus, irrespective of the cue modality, reward modulation exerts similar effects on sensory processing. However, cross-modal and intra-modal reward modulation were not compared directly (but see: Cheng et al., 2020; Vakhrushev et al., 2021).

In our previous study (Vakhrushev et al., 2021), we found differences between intraand cross-modal reward effects on visual sensitivity. In that study, participants performed an orientation discrimination task while the target cue appeared together with visual or auditory cues previously associated with high reward. The results showed improved behavior when target cues appeared together with auditory high-value cues, while visual high-value cues impaired behavioral performance. It was suggested that the difference 
between intra- and cross-modal reward conditions could come from the interaction of reward signals with selective attention (Maunsell, 2004). While the intra-modal reward condition competed for attentional resources with a goal-directed orientation discrimination task (Awh et al., 2012; Failing and Theeuwes, 2018), cross-modal reward cues relied on an independent pool of attentional resources (Keitel et al., 2013), which resulted in improved behavioral and neuronal responses. Alternatively, these differences could be a result of the unique characteristics of visual and auditory modalities. The high spatial sensitivity of visual modality (Klingel et al., 2021; Shams et al., 2000) results in a competition for sensory processing between target and reward cues, while spatially unspecific auditory cues (Halverson, 1927; Slutsky and Recanzone, 2001; Welch and Warren, 1980) exert less competition on sensory processing of the target cue and strengthen the integration between modalities (Bruns et al., 2014; Cheng et al., 2020; Sanz et al., 2018). To resolve these alternatives, cross-modal and intra-modal reward effects should be compared while controlling for the spatial and temporal characteristics of reward cues.

The current study aimed to test intra- and cross-modal reward effects by manipulating visual and auditory modality-specific attentional resources (Keitel et al., 2013). In the first experiment, we controlled attentional resources in visual modality by priming attention to a narrow area or spreading it around the target cue. This manipulation was done as in the experimental paradigm by Castiello and Umilta (1992). There, each trial started by priming the spatial attention by pre-allocating the target cue with frames. The size of the frames changed every trial and varied between small ( $4^{\circ}$ visual degrees), medium ( $7^{\circ}$ visual degrees), and large ( $10^{\circ}$ visual degrees) sizes. In the current study, we modified the previous design by introducing visual (intra-modal) and auditory(cross-modal) task-irrelevant reward cues. Visual cues were a color change of the frame around the target cue. Auditory reward cues were pure tones played together with the appearance of the target cue. We expected to find an interaction between reward and spatial attention as shown in the previous studies (Anderson, 2016a; Anderson et al., 2011a; Anderson and Kim, 2020; Cheng et al., 2020; Kim et al., 2021). Intra- and cross-modal reward cues should improve behavioral performance when spatial attention is allocated narrowly. However, positive effects of intramodal reward cues should disappear when spatial attention is spread, while reward effects from cross-modal reward cues should have similar effects irrespective of the size of the attentional window.

In the second experiment, we examined whether reward effects depend on the temporal synchrony between the reward cues and the target. To test this, we compared intra- and cross- 
modal reward effects when the target and reward cues were presented together with a condition where reward cue preceded the target cue. The experimental design was identical to the first experiment, but frame sizes were fixed over the experiment. We expected to find reward effects only when cue and target appear together and no reward effects when they are separated in time. This result indicates that reward effects are the result of the integration of reward and target cues.

\subsection{Methods: Experiment 1}

\section{Participants}

Forty healthy volunteers participated in Experiment 1 (mean age \pm SD: 25.6 \pm 4.5 ; 24 female). Seven of them were removed after the data collection: 4 participants did not learn the reward associations, 3 participants had accuracies outside the range that we targeted $(\mathrm{N}$ $=2<60 \%$ and $\mathrm{N}=1>95 \%$ ). The remaining 33 participants had a normal or corrected-tonormal vision and normal hearing. The sample size was based on previous studies (Pooresmaeili et al., 2014) and our previous study (Vakhrushev et al., 2021). Participants received 21 Euros as monetary compensation at the end of the experiment. All procedures were approved by “Universitätsmedizin Göttingen” (UMG) under proposal number 15/7/15.

\section{Apparatus}

Experimental stimuli were generated on a Windows computer equipped with MATLAB (version R2015b) and Psychophysics toolbox-3 (Brainard, 1997) and displayed on a 22.5-inch calibrated monitor (ViewPixx/EEG inc., resolution $=1440 \times 980$ pixels, refresh rate $=120 \mathrm{~Hz}$ ). Background luminance, as well as the luminance of cueing colors, were set to $80 \mathrm{~cd} / \mathrm{m}^{2}$. Participants sat $60 \mathrm{~cm}$ away from the screen with their heads fixed on the chinrest. Sounds were delivered via over-ear headphones Sennheiser HDA280 with the loudness of all sounds adjusted to $75 \mathrm{~dB}$. The experiment was done in a sound-attenuated, darkened chamber. 
a)

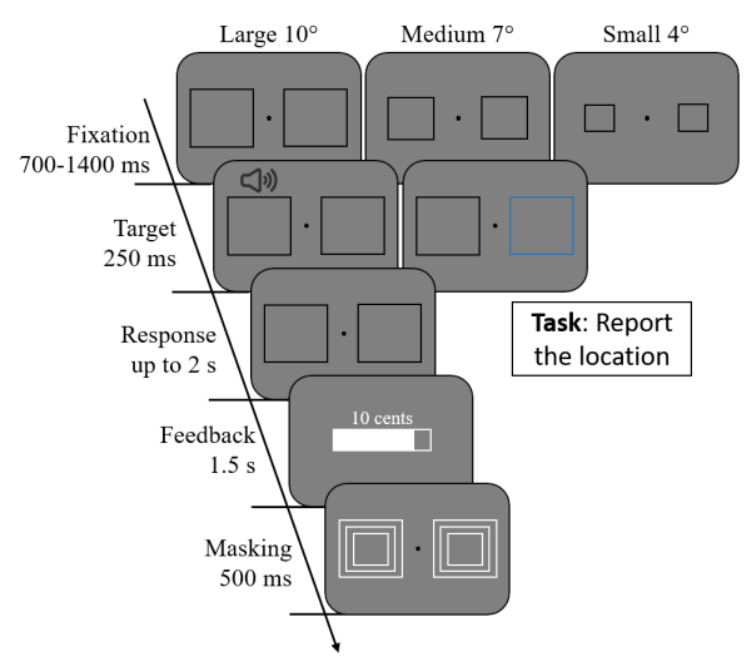

b) Pre- and post-conditioning phases

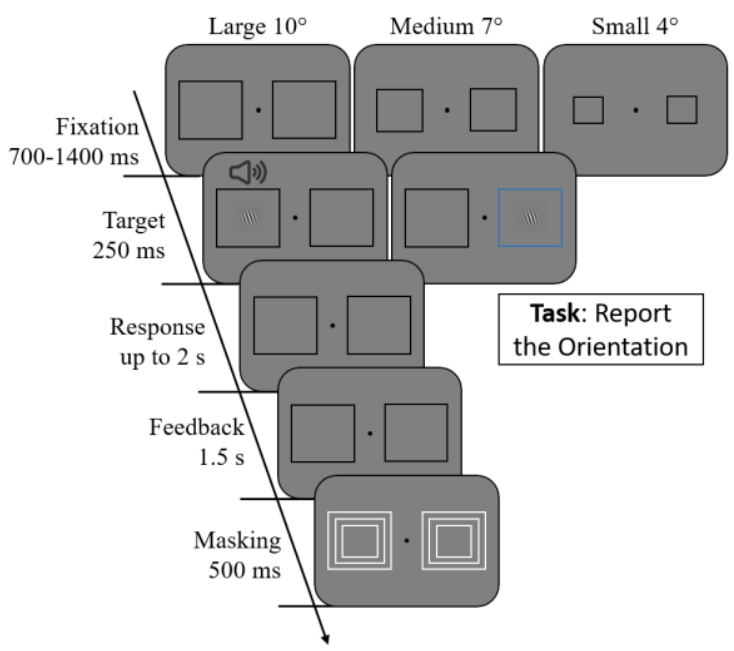

Figure 1. Experiment 1: The sequence of trial events. a) Trial events during the conditioning phase. Participants reported the location of visual or auditory cues. Correct responses were followed by a delivery of monetary reward feedback scaled with the value of the current cue. One visual and one auditory cue systematically delivered more reward, while other delivered less reward. b) Trial events during pre- and postconditioning phases. Participants reported the orientation of the Gabor stimuli inside one of the frames in periphery while ignoring previously rewarding visual and auditory cues. Frames changed the size every trial $\left(4^{\circ}, 7^{\circ}\right.$, or $10^{\circ}$ visual degrees in diameter). The manipulation of the modality, value of the reward cue as well as the spread of attentional window (Castiello and Umiltà, 1992) allowed us to test visual and auditory reward effects on visual sensitivity under different levels of attentional window.

\section{Conditioning phase}

The conditioning phase (Figure 1, left panel) consisted of 288 trials. Each trial started with the fixation period (700-1400 ms), followed by a target period (250 ms), a response period (up to 2 seconds), monetary feedback (1.5 seconds), and a masking window (500 ms). Starting with the fixation period, participants saw a fixation dot $\left(0.1^{\circ} \times 0.1^{\circ}\right.$ visual angle $)$ at the screen center and two identical grey frames $\left(0.1^{\circ}\right.$ visual degrees thick $)$ on the left and right sides of the screen $\left(9^{\circ}\right.$ visual degrees). The size of the frames was manipulated across trials and varied between a small ( $4^{\circ}$ visual degrees), medium ( $7^{\circ}$ visual degrees), and large $\left(10^{\circ}\right.$ visual degrees) size. Next, a visual or auditory target cue appeared. The visual target was a change in the frame color. During the following target period, a visual (a change of frame color) or auditory (a pure tone delivered through an earpiece) cue was presented on the left or right side of the screen $\left(9^{\circ}\right.$ visual degrees). There were two different sounds (350 $\mathrm{Hz}$ or $1050 \mathrm{~Hz}$ ) and two different colors (orange (RGB: 11434 1) or blue (RGB: 061 104)), which delivered high (10 \pm 3 cents) or low $(2 \pm 1$ cent $)$ reward outcomes drawn from a Poisson distribution. Prior to the experiment, sounds and colors were adjusted to reach isoloudness $(75 \mathrm{~dB})$ and isoluminance $\left(60 \mathrm{~cd} / \mathrm{m}^{2}\right)$. The order of the sounds and colors was fully randomized across trials, and value associations were counterbalanced across participants. Participants were instructed to report the side of the cues by pressing "Left" or 
"Right" arrow buttons when the cue appeared on the left or right side of the screen. If reported successfully, participants were given monetary feedback based on the value of the current cue. The feedback consisted of a white numeric output in cents (e.g. " 8 cents") above and a white rectangle frame $\left(3^{\circ}\right.$ width and $1^{\circ}$ height visual angle) under the fixation point. The rectangle was filled from the left to right side with white color proportionally (e.g., 2/3) to the difference between the maximum possible reward (12 cents) and the monetary reward of the current trial. Each trial ended with a masking display intended to remove any possible afterimage of the lateralized frames. For this reason, at the location of the frames displayed on the left and right sides of the screen, identical frames of all possible sizes appeared in white color.

\section{Pre- and post-conditioning phases}

In the pre- and post-conditioning phases (Figure 1, right panel), participants were required to perform a visual orientation discrimination task. Similar to the conditioning phase, trials in these phases started with a fixation period (700-1400 ms) with the fixation dot at the screen center and two frames on the left and right sides $\left(9^{\circ}\right.$ visual degrees) with three different sizes $\left(4^{\circ}, 7^{\circ}\right.$, or $10^{\circ}$ visual degrees $)$ across trials. The fixation period was followed by the presentation of a Gabor patch (target, $250 \mathrm{~ms}$ ) with a near-threshold tilt angle. The Gabor consisted of vertically aligned Gaussian-windowed sinusoidal gratings with an $\mathrm{SD}$ of $0.33^{\circ}$, a spatial frequency of 3 cycles per degree, $2^{\circ}$ diameter, and $50 \%$ contrast. Simultaneously with the Gabor, a change of frames' color (visual cue) or the presentation of a sound (auditory cue) occurred ipsilateral to it in all trials except for trials containing a neutral condition. Participants were instructed to report the orientation of the Gabor patch by pressing "Left" arrow button when the Gabor had counter clock-wise orientation or "Right" arrow button when the Gabor had clock-wise orientation and ignored visual or auditory cues. The response window was set to 2 seconds. Responses within the response window were followed by a fixation screen for 1.5 seconds and a masking period for $500 \mathrm{~ms}$. Trials with responses slower than 2 seconds and trials where participants made excessive eye movements ( $>2^{\circ}$ from the fixation point) were repeated in an additional block at the end of the experiment. Similar to the conditioning phase, the masking window included the presentation of peripheral ( $9^{\circ}$ visual degrees) white frames with $4^{\circ}, 7^{\circ}$ and $10^{\circ}$ (visual angle) sizes. 


\section{Procedure}

The experiment began with a training phase including 30 trials of the orientation discrimination task with the Gabor presented at a tilt angle of $\pm 45^{\circ}$. In the following threshold phase, the tilt angle of the Gabor patch was adjusted for each participant to achieve $70 \%$ accuracy using a QUEST procedure (Watson and Pelli, 1983). After completing the threshold phase, participants completed the pre-conditioning phase, where we measured the baseline performance of the Gabor orientation discrimination, followed by the conditioning phase and 24 mini-blocks of interleaved conditioning and post-conditioning phases.

The total, there were 936 trials. In the pre-conditioning phase participants completed 216 trials (16 repetitions per reward condition 8 repetitions for neutral condition), and in post-conditioning 432 trials (32 repetitions per reward condition and 16 repetitions for neutral condition). The conditioning phase consisted of 96 trials where reward associations were learned. An additional 192 trials of conditioning phase were divided into 24 miniblocks ( 8 trials per block). These blocks were interleaved with blocks from the postconditioning phase to prevent the extinction of reward associations (hence in total 24 trials per condition).

\section{Data analysis}

The behavioral effects were assessed by measuring the reaction time (RT, correct trials only) and d-prime (d'). RT was measured from the onset of the target cue until the response. Trials with speeded responses ( $<10 \mathrm{~ms}$ ) and RT with more than two standard deviations above or below the mean for each condition for each phase for each participant were removed (Anderson and Yantis, 2013). In total, $5.30 \pm 1.04 \%$ trials were removed across participants. D' was derived from the accuracy with the formula: $\mathrm{d}^{\prime}=\mathrm{Z}\left(\mathrm{P}_{\mathrm{Hit}}\right)-\mathrm{Z}\left(\mathrm{P}_{\mathrm{FA}}\right)$, where one of the tilt directions was arbitrarily treated as "target-present" as formal Signal Detection Theory analysis of discrimination tasks (Leo et al., 2011; Macmillan and Creelman, 1991). Extreme values of $\mathrm{P}_{\mathrm{Hit}}$ or $\mathrm{P}_{\mathrm{FA}}$ (i.e., a probability equal to 0 or 1 ) were adjusted to 0.001 and 0.999 , respectively. Analysis of the post-conditioning phase was done by subtracting the preconditioning phase from the post-conditioning phase to control for participants' perceptual biases for different visual and auditory cues that were independent of their reward value. Significance was defined as $p<0.05$. All statistical analysis was conducted using MATLAB $2015 b$.

In total, there were three independent variables: cue modality (visual or auditory cues), cue value (high-value or low-value cues), and frame size $\left(4^{\circ}, 7^{\circ}\right.$, and $\left.10^{\circ}\right)$. The neutral 
condition in which the Gabor stimulus was not accompanied by a change in frames' color or a sound was inspected separately to assess participants' performance irrespective of rewards.

\subsection{Results: Experiment 1}

\section{Effects of the size of attentional focus}

First, we examined whether the size of the attentional window $\left(2^{\circ}, 7^{\circ}\right.$, and $10^{\circ}$ visual degrees) produces a reliable attentional modulation before the learning of reward associations (pre-conditioing phase). For this the data was averaged across visual and auditory conditions and an ANOVA was performed with factors cue type (neutral condition, visual cues, auditory cues) and frame size $\left(4^{\circ}, 7^{\circ}\right.$ and $\left.10^{\circ}\right)$. The ANOVA showed a significant effect of cue type $\mathrm{F}(2,64)=15.69, p<0.001, \eta_{\mathrm{p}}{ }^{2}=0.33$ and no effect of frame size $\mathrm{F}(2,64)=1.45, p=0.24$ or an interaction effect $\mathrm{F}(4,128)=0.54, p=0.65$. Following pairwise comparisons revealed higher visual sensitivity (mean \pm s.e.m.: $4.27 \pm 0.22$ ) in nocue condition compared to conditions when the sound was played (mean \pm s.e.m.: $3.43 \pm$ $0.18)\left(\mathrm{t}(32)=4.39, p<0.001, \mathrm{~d}_{\mathrm{z}}=0.76\right)$ or visual cue was displayed (mean \pm s.e.m.: $3.27 \pm$ $0.21)\left(\mathrm{t}(32)=4.36, p<0.001, \mathrm{~d}_{\mathrm{z}}=0.76\right)$. Similar two way ANOVA with reaction times (RTs) as independent variable did not reveal any significant effect: cue type effect $\mathrm{F}(2,64)=$ $2.19, p=0.14$, frame size $\mathrm{F}(2,64)=0.50, p=0.58$ or their interaction $\mathrm{F}(4,128)=0.81, p=$ 0.46. There results contrast with our expectations and previous findings (Castiello and Umiltà, 1992), where reaction times revealed a significant main effect. We suggest that the lack of frame size effects could be attributed to the difference between tasks participants performed. In previous study by Castiello and Umiltà (1992) participants performed a detection task, while in our experiment participants performed an orientation discrimination task. Importantly, these results indicate that the auditory and visual cues acted as distractors and significantly interfered with the processing of the target. We next asked how reward value affects the impact of auditory and visual cues on the target processing.

\section{Reward effects under different sizes of attentional focus}

Next, we compared the effects of intra- and cross-modal reward cues under different levels of the attentional window. Analysis of d' with a three-way ANOVA (Modality $x$ Value $\times$ Frame size) revealed no main effect of value $(\mathrm{F}(1,32)=1.00, p=0.32)$, no main effect of modality $(\mathrm{F}(1,32)=0.30, p=0.58)$, and no effect of frame size $(\mathrm{F}(2,64)=0.09, p$ $=0.91$ ). However the analysis revealed an interaction between factors modality and value $\left(\mathrm{F}_{(1,35)}=4.22, p<0.05, \eta_{\mathrm{p}}{ }^{2}=0.15\right)$. Following up on the observed interaction effect, we 
conduct $2 \times 2$ ANOVAa at each frame size $\left(4^{\circ}, 7^{\circ}\right.$, and $\left.10^{\circ}\right)$. This analysis revealed an interaction effect between modality and value when the frame size was $10^{\circ}\left(\mathrm{F}_{(1,32)}=5.34, p\right.$ $\left.<0.05, \eta_{\mathrm{p}}{ }^{2}=0.14\right)$, where a higher reward in auditory cue improved the visual sensitivity (mean \pm s.e.m.: $1.17 \pm 0.36$ ) whereas higher reward in visual modality decreased it (mean \pm s.e.m.: $-0.01 \pm 0.46$ ). Pairwise comparisons revealed that across the pre and postconditioning phases, high-value auditory cue had more positive effect on visual sensitivity (mean \pm s.e.m.: $-0.31 \pm 0.27$ ) compared to a low-value auditory cue (mean \pm s.e.m.: $-1.48 \pm$ 0.29), whitch decreased the visual sensitivity after participants learned reward associations $\left(\mathrm{t}(32)=3.28, p<0.01, \mathrm{~d}_{\mathrm{z}}=0.57\right)$.
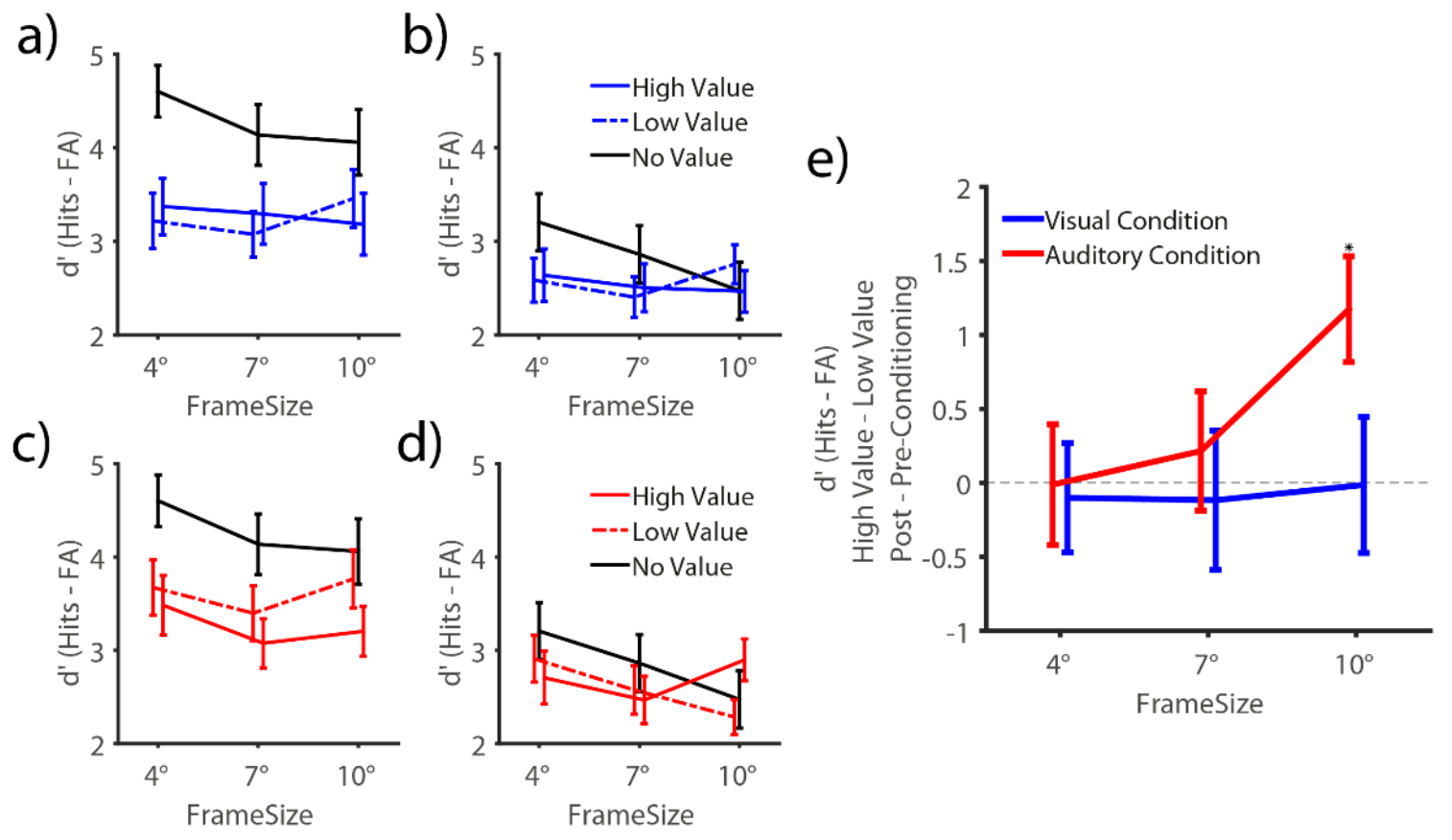

Figure 3. Experiment 1: The effects of visual and auditory reward cues on visual sensitivity (d': hits false alarms) when the focus of attention was narrow ( $4^{\circ}$ visual degrees), spread $\left(10^{\circ}\right.$ visual degrees), or in between ( $7^{\circ}$ visual degrees). a) d' during preconditioning phase showed the difference in performance when the target cue was presented alone (black line) compared to when it presented with a visual cue (blue, solid, and dashed lines) b) d' during the post-conditioning phase show similar differences between condition without a visual cue (black line) and conditions with high- (solid blue line) and low-value (dashed blue line) visual cues. c) d' during the pre-conditioning phase when target cue was displayed together with the auditory cue (red, solid and dashed lines). d) d' during post-conditioning phase reflects a change in performance when the attentional window was spread $\left(10^{\circ}\right.$ visual degrees) compared to a similar condition in the pre-conditioning phase. e) Reward effects that emerge after learning reward associations in conditioning phase (pre-conditioning phase subtracted from post-conditioning phase). The results showed a significant increase in visual sensitivity by a high-value auditory cue when the attentional was spread $\left(10^{\circ}\right.$ visual degrees $)$.

Analysis of RTs with a three way ANOVA (Modality $\times$ Value $\times$ Frame size) revealed a two-way interaction effect between Modality and Value $\left(\mathrm{F}_{(1,32)}=4.75, p=0.037, \eta_{\mathrm{p}}{ }^{2}=\right.$ $0.13)$. Main effect of modality $\mathrm{F}(1,32)=2.40, p=0.13$, frame size $\mathrm{F}(2,64)=0.28, p=0.74$ and value $\mathrm{F}(1,32)=1.75, \mathrm{p}=0.19$ did not reach significance level. Analysis of effects for each level of frame size revealed that the interaction effect between value and modality 
$\left(\mathrm{F}_{(1,32)}=7.36, p=0.011, \eta_{\mathrm{p}}^{2}=0.19\right)$ was significant when the frame size was small $\left(4^{\circ}\right) . \mathrm{At}$ this frame size $\left(4^{\circ}\right)$, reward cues in visual modality sped up participants' responses (mean \pm s.e.m.: $-32.36 \pm 14.85 \mathrm{~ms}$ ) compared to reward cues in auditory modality (mean \pm s.e.m.: $49.55 \pm 28.03 \mathrm{~ms})$. Additionally, planned pairwise comparisons revealed a difference between high- and low- value visual cues when the attention frame size was narrow $(\mathrm{t}(32)=$ $2.18, p=0.037, d_{z}=0.38$ ). High value visual cues lowered the speed of responses (mean \pm s.e.m.: $-84.41 \pm 18.42$ ) compared to a low value visual cue (mean \pm s.e.m.: $-52.05 \pm 19.61$ ).

When the frame size was $7^{\circ}$ a main effect of modality reached significance $(\mathrm{t}(32)=2.2, p<$ $0.05, \mathrm{~d}_{\mathrm{z}}=0.38$ ). Responses to visual cues were slower (mean \pm s.e.m.: $-54.06 \pm 15.34 \mathrm{~ms}$ ) compared to auditory cues (mean \pm s.e.m.: $-85.55 \pm 14.99 \mathrm{~ms}$ ).

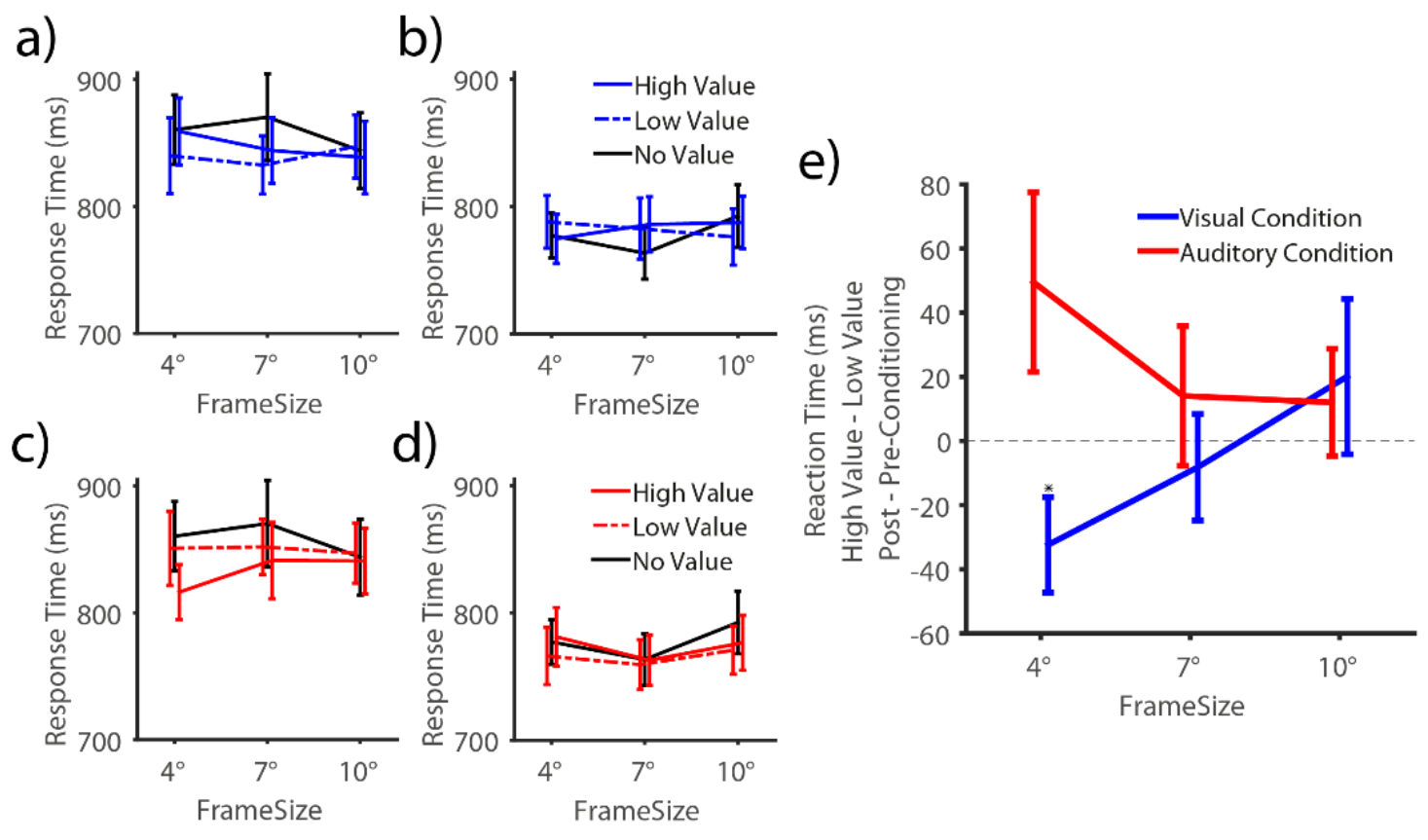

Figure 4. Experiment 1: Modulation of reaction times (correct trials) by a) visual cues before conditioning, b) visual cues after conditioning, c) auditory cues before conditioning, d) auditory cues after conditioning. e) Commutative reward effects of visual and auditory on response time (post-conditioning minus preconditioning). The results revealed that high-value visual cues improved response time significantly when the attention was focused narrowly on the target cue $\left(4^{\circ}\right)$. Auditory cues on another size decreased response time when the attention was narrow.

\section{Interim discussion}

The first experiment aimed to compare intra- and cross-modal reward effects when spatial attention was wide or narrow around the target cue. Results from the pre- and postconditioning phases showed a significant difference in visual sensitivity between different levels of spatial attention, where behavioral performance was higher in narrow conditions. However, we did not find attentional effects in the reaction times, which was reported before (Castiello and Umiltà, 1992). Lack of attentional effects in response time could be due to the 
difference in the experimental task from the original study since the current experiment used an orientation discrimination task, while this previous study used a detection task. We use the main effect of the frame size as a reason to test effects of modality and value for each attentional window.

The analysis of intra- and cross-modal reward effects across pre- and post-conditioning phases revealed modulation of response times when the focus of attention was narrow and modulation of visual sensitivity when the focus attention was wide. These effects were specific for intra-modal and cross-modal conditions, where intra-modal reward cues improved response times when the focus of attention was narrow, while cross-modal reward cues improved visual sensitivity when the focus of attention was spread. These results indicated that attention capture by intra-and cross-modal reward cues occurs under different sizes of attentional focus. Intra-modal reward cues captured attention to the reward cues and the target (Anderson et al., 2011; Kim et al., 2021), thus facilitating reaction time when the focus of attention is narrow. Moreover, cross-modal reward cues enhanced visual sensitivities (d') when the focus of attention is wide ( $10^{\circ}$ visual degrees). However, the exact mechanisms of the latter effect are unclear and have not been investigated before. One possibility is that high reward enhances the audiovisual integration between the target and the task-irrelevant sounds. This way, high reward can improve the processing of target, either by reducing the distracting effect of the sounds or by enhancing the target representation directly. To examine this possibility, we conducted a second experiment where a wide focus of attention (frame size $=10^{\circ}$ ) at which cross-modal effects were maximum was employed and at the same time we manipulated the asynchrony (SOA) of the reward cues. If the integration between target and reward cues accounts for our effects, a dependence on the SOA would be expected. On the other hand, if our effects are due to an unspecific increase in alertness, no dependence on SOA would occur.

\subsection{Methods: Experiment 2}

In total, 36 participants participated in the experiment. Four participants were removed. Three participants did not learn reward associations, and 1 participant had below chance level performance $(<50 \%)$.

The second experiment had the same experimental procedure as the first experiment. The experiment started with the training, and threshold estimation, followed by the preconditioning phase, conditioning phase, and postconditioning phase, blocks of which were interleaved with blocks from the conditioning phase. Notably, the size of the frames at the 
beginning of the trials was fixed to a large size $\left(10^{\circ}\right)$. This choice was motivated by two reasons. Firstly, our results showed an effect of high-value auditory cues that improved visual sensitivity when the frame size was spread. Secondly, we interpreted the lack of reward effects on visual sensitivity in high-value visual cue conditions due to sensory competition between visual target and visual reward cue. This was especially evident when the attention narrowed where high-value cues increased response time but not affect visual sensitivity. Thus, for the second experiment we tried to minimize sensory competition that we observe in visual high-value conditions and to replicate positive effects of auditory highvalue cues on visual sensitivity. Since there were no changes in frame size, we removed the masking period at the end of the trial. Importantly, we manipulated the Stimuli Onset Latency (SOA) between reward stimuli and the target. There were four SOAs: $-150,-50,0$, $50 \mathrm{~ms}$.

\subsection{Results: Experiment 2}
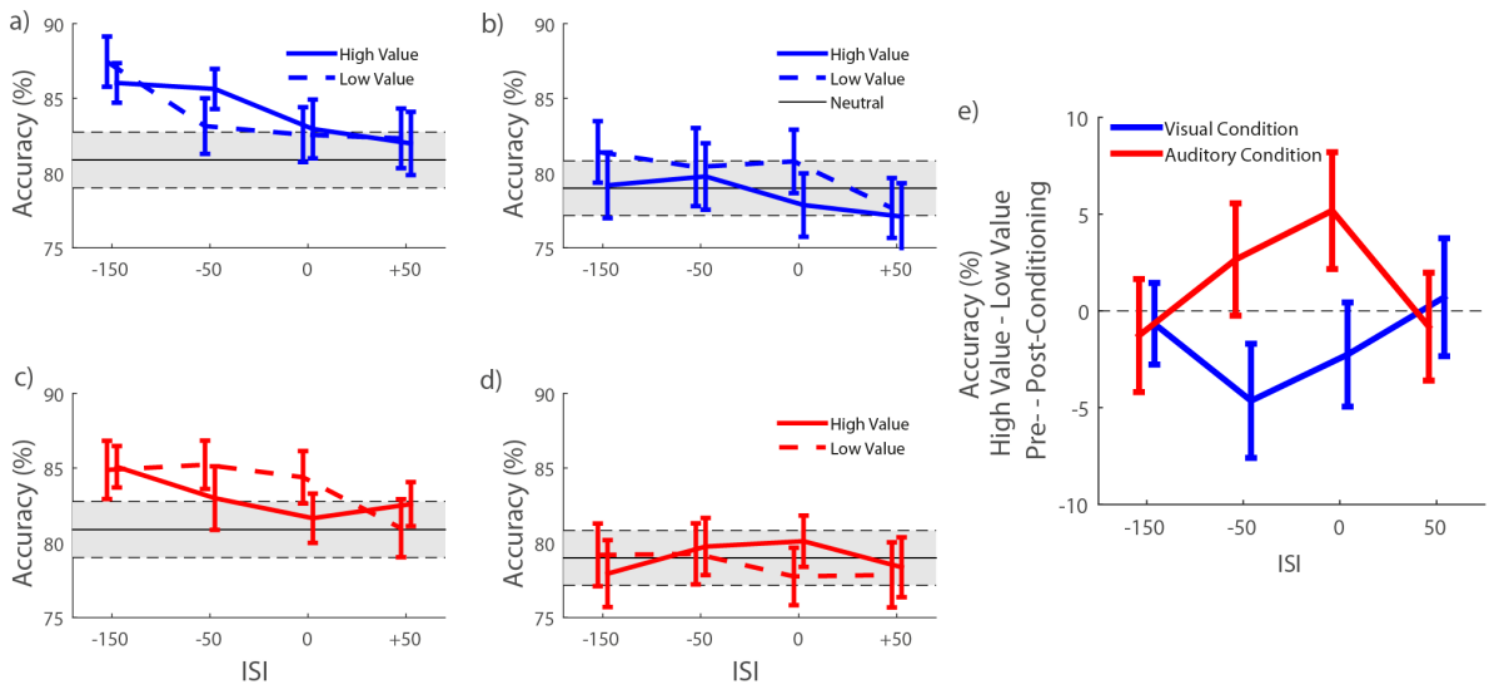

Figure 4. Experiment 2: Modulation of the accuracy of visual orientation discrimination by a) visual cues before conditioning b) visual cues after conditioning, c) auditory cues before conditioning, d) auditory cues after conditioning. e) Commutative reward effects of visual and auditory on accuracy (post-conditioning minus pre-conditioning). The results revealed an interaction between visual and auditory reward effects when reward cues appeared together with the target $(0 \mathrm{~ms})$. Reward value of auditory cue improved accuracy during the orientation discrimination task. Reward value of visual cues instead affected accuracy negatively. These results indicate that previously reported reward effects in experiment 1 (see also: Vakhrushev et al., 2021) reflect the integration between reward and target cues. Additionally, suppression effects that were reported in intra-modal condition could not be explained by perceptual competition since reward effects were the same between synchronous condition $(0 \mathrm{~ms})$ and when target and reward cues were slightly separated in time $(-50 \mathrm{~ms})$.

First, we measured cueing effect during pre-conditioning phase across different SOA levels $(-150,-50,0,50 \mathrm{~ms})$. Here reward conditions were averaged for each cue type (visual, auditory, neutral). A two-way ANOVA revealed a significant SOA effect $\mathrm{F}(3,93)=3.82, p$ $=0.014, \eta_{\mathrm{p}}{ }^{2}=0.11$. Participants were more accurate at reporting the orientation when the 
target location was cued $150 \mathrm{~ms}$ before the target onset (mean \pm s.e.m.: $84.16 \pm 1.13$ ) compared to a condition when cues appear together (mean \pm s.e.m.: $82.26 \pm 1.37)(\mathrm{t}(31)=$ 2.3, $p=0.028, \mathrm{~d} z=0.41)$ or when cue follows the target (mean \pm s.e.m.: $81.76 \pm 1.48)(\mathrm{t}(31)$ $\left.=3.1, p=0.004, \mathrm{~d}_{\mathrm{z}}=0.55\right)$.

Next, we tested whether reward effects depend on the allocation of spatial attention. For this, we measured reward effects across different SOA levels (-150, -50, 0, $50 \mathrm{~ms})$ where pre- and post-conditioning phases were subtracted. A three-way ANOVA with factors SOA, modality (visual, auditory) and value (high reward, low reward) revealed an interaction effect between cue modality and cue value $\left(\mathrm{F}_{(1,31)}=5.56, p<0.05, \eta_{\mathrm{p}}{ }^{2}=0.15\right)$. Follow up ANOVAs at each SOA revealed that the interaction effect between modality and value was significant at $\mathrm{SOA}=0$, i.e., when target and reward cues were synchronized $\left(\mathrm{F}_{(1,31)}=5.83\right.$, $\left.p<0.05, \eta_{\mathrm{p}}^{2}=0.16\right)$. At this $\mathrm{SOA}$, a high value in auditory modality improved the orientation discrimination accuracy (mean \pm s.e.m.: $0.05 \pm 0.03$ ). At the same time, high-value visual cues decreased the visual sensitivity (mean \pm s.e.m.: $-0.02 \pm 0.03$ ). The effect in individual modalities did not reach significance (all ps >0.1).

Analysis of reaction times and d' with three-way ANOVA did not reveal any significant effect of modality, value, SOA, or their interaction (all ps >0.1).

\subsection{Discussion}

The current study compared intra- and cross-modal reward effects with respect to the spatial (experiment 1) and temporal (experiment 2) characteristics of attentional focus. Results from the first experiment revealed different reward effects between intra- and crossmodal reward cues. When the focus of attention was narrow, intra-modal reward cues sped up responses. This result is consistent with previous studies (Anderson et al., 2011a), where it has been shown that reward cues capture attention and impair response time when reward cue appears as a distractor and improve response time when appear as a target or in the target location. In our case, the capture of attention by visual reward cues (color change of frames) potentially also enhanced the processing of the Gabor that was adjacent but not overlapping with the reward cues. On another side high-value cross-modal cues improved sensory processing of visual targets, but only when the focus of attention was spread. Reward effects in the cross-modal condition are consistent with previous findings that showed enhanced visual sensitivity by reward-value auditory cues (Leo and Noppeney, 2014; Pooresmaeili et al., 2014; Vakhrushev et al., 2021). The difference between intra- and cross-modal effects 
and their interaction with spatial attention suggests that these reward effects follow different modulation pathways.

Recent studies have suggested that reward value may promote the integration of information across sensory modalities (Bruns et al., 2014; Cheng et al., 2020). To test whether such a mechanism can account for the effects we observed in experiment 1 or instead are due to priming of attention, in the second experiment, we controlled the temporal synchronization of the target and the reward cues. Here, reward cues either preceded or followed the target or were presented synchronously. The results replicated our results at the large frame size in Experiment 1 and additionally showed that cross-modal enhancement of visual perception maximally occurs at $\mathrm{SOA}=0$ hence suggesting that they are likely due to the enhanced integration of reward cues and the target. When we compared intra- and crossmodal reward effects we found an interaction effect, where intra-modal reward cues impaired behavioral performance while cross-modal cues improved it. This finding replicates our previous results (Vakhrushev et al., 2021).

In both experiments, task-irrelevant auditory and visual cues were found to interfere with the target processing compared to a neutral condition where the Gabor target was presented alone. This indicates that the observed behavioral advantages of intra- and crossmodal cues primarily occur due to reduced interference of potentially distracting stimuli with the target. This is in line with the proposal of recent studies suggesting that motivational signals may overall enhance the noise resistance of the sensory (Arsenault et al., 2013; Goltstein et al., 2018) and sensorimotor systems (Manohar et al., 2019, 2015). We furthered these studies by showing that by presenting reward cues at their optimal size of attentional focus, i.e. narrow for intra-modal and wide for cross-modal cues, attentional filtering of unwanted or irrelevant information could be boosted. Additionally, when the focus of attention was wide and sensory representations of the target were weak, reward enhanced the gain of sensory representations by boosting the integration of the target and the reward cues, thereby allowing the privileged processing of reward cues to extend to the target. These two mechanisms, i.e. improving the filtering of distractors and enhancing the representation of targets, can conjointly underly the reward-driven modulation of perception. Future studies will be needed to test the unique contribution of these factors to reward effects.

In summary, we showed that the effects of previously rewarded, task-irrelevant cues on visual perception depends on the focus of attention and the sensory modality of the reward cues and that the cross-modal and intra-modal reward cues exert their effects under different sizes of the attentional focus. A combination of enhancing distractor filtering or boosting 
target representations through an increase of grouping between reward cues and target may account for these results.

\section{Acknowledgments}

We thank Annika Ziereis for her help with the data collection and suggestions regarding the design. This work was supported by an ERC Starting Grant (no: 716846) to AP.

\section{Authors' contributions}

$\mathrm{RV}$ and AP conceptualized, designed the experiment, and participated in the preregistration of the study. RV conducted the experiment. All authors analyzed the data, interpreted the results, and wrote the first draft of the manuscript. All authors revised the manuscript. 


\subsection{References}

Ainslie, G., 1975. Specious reward: A behavioral theory of impulsiveness and impulse control. Psychol. Bull. 82, 463-496. https://doi.org/10.1037/h0076860

Alais, D., Morrone, C., Burr, D., 2006. Separate attentional resources for vision and audition. Proc. R. Soc. B Biol. Sci. 273, 1339-1345. https://doi.org/10.1098/rspb.2005.3420

Algazi, U., Duda, R., Thompson, D., The, C.A.-2001 I.W. on, 2001. The CIPIC HRTF Database, in Applications of Signal Processing to Audio and Acoustics. IEEE Work. on the, 99-102.

Alsius, A., Navarra, J., Campbell, R., Soto-Faraco, S., 2005. Audiovisual integration of speech falters under high attention demands. Curr. Biol. 15, 839-843. https://doi.org/10.1016/j.cub.2005.03.046

Anderson, B.A., 2019. Neurobiology of value-driven attention. Curr. Opin. Psychol. https://doi.org/10.1016/j.copsyc.2018.11.004

Anderson, B.A., 2017. Reward processing in the value-driven attention network: reward signals tracking cue identity and location. Soc. Cogn. Affect. Neurosci. 12, 461-467. https://doi.org/10.1093/scan/nsw141

Anderson, B.A., 2016a. Value-driven attentional capture in the auditory domain. Attention, Perception, Psychophys. 78, 242-250. https://doi.org/10.3758/s13414-015-1001-7

Anderson, B.A., 2016b. The attention habit: how reward learning shapes attentional selection. Ann. N. Y. Acad. Sci. 1369, 24-39. https://doi.org/10.1111/nyas.12957

Anderson, B.A., 2013. A value-driven mechanism of attentional selection. J. Vis. 13, 7-7. https://doi.org/10.1167/13.3.7

Anderson, B.A., Kim, A.J., 2020. Selection history-driven signal suppression. Vis. cogn. https://doi.org/10.1080/13506285.2020.1727599

Anderson, B.A., Kim, H., Kim, A.J., Liao, M.-R., Mrkonja, L., Clement, A., Grégoire, L., 2021. The Past, Present, and Future of Selection History. Neurosci. Biobehav. Rev. 130, 326-350. https://doi.org/10.1016/j.neubiorev.2021.09.004

Anderson, B.A., Laurent, P.A., Yantis, S., 2011a. Value-driven attentional capture. Proc. Natl. Acad. Sci. U. S. A. 108, 10367-10371. https://doi.org/10.1073/pnas.1104047108

Anderson, B.A., Laurent, P.A., Yantis, S., 2011b. Learned Value Magnifies Salience-Based Attentional Capture. PLoS One 6, e27926. https://doi.org/10.1371/journal.pone.0027926

Anderson, B.A., Yantis, S., 2013. Persistence of value-driven attentional capture. J. Exp. Psychol. Hum. Percept. Perform. 39, 6-9. https://doi.org/10.1037/a0030860

Anderson, Laurent, Yantis, Anderson, B.A., Laurent, P.A., Yantis, S., 2013. Reward predictions bias attentional selection. Front. Hum. Neurosci. 7, 1-6. https://doi.org/10.3389/fnhum.2013.00262

Arsenault, J.T., Nelissen, K., Jarraya, B., Vanduffel, W., 2013. Dopaminergic Reward Signals Selectively Decrease fMRI Activity in Primate Visual Cortex. Neuron 77, 1174-1186. https://doi.org/10.1016/j.neuron.2013.01.008

Asutay, E., Västfjäll, D., 2016. Auditory attentional selection is biased by reward cues. Sci. Rep. 6, 1-6. https://doi.org/10.1038/srep36989

Awh, E., Belopolsky, A. V., Theeuwes, J., 2012. Top-down versus bottom-up attentional control: A failed theoretical dichotomy. Trends Cogn. Sci. https://doi.org/10.1016/j.tics.2012.06.010

Bachman, M.D., Wang, L., Gamble, M.L., Woldorff, M.G., 2020. Physical Salience and ValueDriven Salience Operate through Different Neural Mechanisms to Enhance Attentional Selection. J. Neurosci. 40, 5455-5464. https://doi.org/10.1523/JNEUROSCI.1198-19.2020

Baines, S., Ruz, M., Rao, A., Denison, R., Nobre, A.C., 2011. Modulation of neural activity by motivational and spatial biases. Neuropsychologia 49, 2489-2497. https://doi.org/10.1016/j.neuropsychologia.2011.04.029

Balan, P.F., Gottlieb, J., 2006. Integration of exogenous input into a dynamic salience map revealed by perturbing attention. J. Neurosci. 26, 9239-9249. https://doi.org/10.1523/JNEUROSCI.1898-06.2006

Baldassi, S., Simoncini, C., 2011. Reward sharpens orientation coding independently of attention. Front. Neurosci. 5, 13. https://doi.org/10.3389/fnins.2011.00013

Bao, S., Chan, V.T., Merzenich, M.M., 2001. Cortical remodelling induced by activity of ventral 
tegmental dopamine neurons. Nature 412, 79-83. https://doi.org/10.1038/35083586

Basten, U., Biele, G., Heekeren, H.R., Fiebach, C.J., 2010. How the brain integrates costs and benefits during decision making. Proc. Natl. Acad. Sci. U. S. A. 107, 21767-21772. https://doi.org/10.1073/pnas.0908104107

Bayer, M., Grass, A., Schacht, A., 2019. Associated valence impacts early visual processing of letter strings: Evidence from ERPs in a cross-modal learning paradigm. Cogn. Affect. Behav. Neurosci. 19, 98-108. https://doi.org/10.3758/s13415-018-00647-2

Bayer, M., Rossi, V., Vanlessen, N., Grass, A., Schacht, A., Pourtois, G., 2017. Independent effects of motivation and spatial attention in the human visual cortex. Soc. Cogn. Affect. Neurosci. 12, 146-156. https://doi.org/10.1093/scan/nsw162

Bayer, M., Sommer, W., Schacht, A., 2012. P1 and beyond: Functional separation of multiple emotion effects in word recognition. Psychophysiology 49, 959-969. https://doi.org/10.1111/j.1469-8986.2012.01381.x

Bean, N.L., Stein, B.E., Rowland, B.A., 2021. Stimulus value gates multisensory integration. Eur. J. Neurosci. 53, 3142-3159. https://doi.org/10.1111/ejn.15167

Berridge, K.C., 2004. Motivation concepts in behavioral neuroscience. Physiol. Behav. 81, 179-209. https://doi.org/10.1016/J.PHYSBEH.2004.02.004

Berridge, K.C., 2000. Reward learning: Reinforcement, incentives, and expectations. Psychol. Learn. Motiv. - Adv. Res. Theory 40, 223-278.

Berridge, K.C., Kringelbach, M.L., 2015. Pleasure Systems in the Brain. Neuron 86, 646-664. https://doi.org/10.1016/J.NEURON.2015.02.018

Berridge, K.C., Kringelbach, M.L., 2008. Affective neuroscience of pleasure: Reward in humans and animals. Psychopharmacology (Berl). https://doi.org/10.1007/s00213-008-1099-6

Berridge, K.C., Robinson, T.E., 1998. What is the role of dopamine in reward: Hedonic impact, reward learning, or incentive salience? Brain Res. Rev. 28, 309-369. https://doi.org/10.1016/S0165-0173(98)00019-8

Bertelson, P., Radeau, M., 1981. Cross-modal bias and perceptual fusion with auditory-visual spatial discordance. Percept. Psychophys. 29, 578-584. https://doi.org/10.3758/BF03207374

Bisley, J.W., 2011. The neural basis of visual attention. J. Physiol. https://doi.org/10.1113/jphysiol.2010.192666

Bolognini, N., Frassinetti, F., Serino, A., Làdavas, E., 2005. “Acoustical vision” of below threshold stimuli: Interaction among spatially converging audiovisual inputs. Exp. Brain Res. 160, 273282. https://doi.org/10.1007/s00221-004-2005-z

Bourgeois, A., Chelazzi, L., Vuilleumier, P., 2016. How motivation and reward learning modulate selective attention. Prog. Brain Res. 229, 325-342. https://doi.org/10.1016/BS.PBR.2016.06.004

Brainard, D.H., 1997. The Psychophysics Toolbox. Spat. Vis. 10, 433-436. https://doi.org/10.1163/156856897X00357

Braver, T.S., Krug, M.K., Chiew, K.S., Kool, W., Andrew Westbrook, J., Clement, N.J., Alison Adcock, R., Barch, D.M., Botvinick, M.M., Carver, C.S., Cools, R., Custers, R., Dickinson, A., Dweck, C.S., Fishbach, A., Gollwitzer, P.M., Hess, T.M., Isaacowitz, D.M., Mather, M., Murayama, K., Pessoa, L., Samanez-Larkin, G.R., Somerville, L.H., 2014. Mechanisms of motivation-cognition interaction: Challenges and opportunities. Cogn. Affect. Behav. Neurosci. https://doi.org/10.3758/s13415-014-0300-0

Bruns, P., 2019. The Ventriloquist Illusion as a Tool to Study Multisensory Processing: An Update. Front. Integr. Neurosci. https://doi.org/10.3389/fnint.2019.00051

Bruns, P., Maiworm, M., Röder, B., 2014. Reward expectation influences audiovisual spatial integration. Attention, Perception, Psychophys. 76, 1815-1827. https://doi.org/10.3758/s13414-014-0699-y

Busse, L., Roberts, K.C., Crist, R.E., Weissman, D.H., Woldorff, M.G., 2005. The spread of attention across modalities and space in a multisensory object. Proc. Natl. Acad. Sci. U. S. A. 102, 18751-18756. https://doi.org/10.1073/pnas.0507704102

Bustamante, L., Lieder, F., Musslick, S., Shenhav, A., Cohen, J., 2021. Learning to Overexert Cognitive Control in a Stroop Task. Cogn. Affect. Behav. Neurosci. 1-19. https://doi.org/10.3758/s13415-020-00845-x

Calabresi, P., Picconi, B., Tozzi, A., Di Filippo, M., 2007. Dopamine-mediated regulation of 
corticostriatal synaptic plasticity. Trends Neurosci. https://doi.org/10.1016/j.tins.2007.03.001

Calvert, G.A., Thesen, T., 2004. Multisensory integration: Methodological approaches and emerging principles in the human brain. J. Physiol. Paris 98, 191-205. https://doi.org/10.1016/j.jphysparis.2004.03.018

Carrasco, M., 2011. Visual attention: The past 25 years. Vision Res. 51, 1484-1525. https://doi.org/10.1016/J.VISRES.2011.04.012

Castiello, U., Umiltà, C., 1992. Splitting Focal Attention. J. Exp. Psychol. Hum. Percept. Perform. 18, 837-848. https://doi.org/10.1037/0096-1523.18.3.837

Chang, S., Egeth, H.E., 2021. Can salient stimuli really be suppressed? Attention, Perception, Psychophys. 83, 260-269. https://doi.org/10.3758/s13414-020-02207-8

Chelazzi, L., Eštočinová, J., Calletti, R., Gerfo, E. Lo, Sani, I., Libera, C. Della, Santandrea, E., 2014. Altering spatial priority maps via reward-based learning. J. Neurosci. 34, 8594-8604. https://doi.org/10.1523/JNEUROSCI.0277-14.2014

Chelazzi, L., Perlato, A., Santandrea, E., Della Libera, C., 2013. Rewards teach visual selective attention. Vision Res. 85, 58-72. https://doi.org/10.1016/J.VISRES.2012.12.005

Cheng, Saglam, A., André, S., Pooresmaeili, A., 2020. Cross-Modal Integration of Reward Value during Oculomotor Planning. eneuro 7, ENEURO.0381-19.2020. https://doi.org/10.1523/ENEURO.0381-19.2020

Corbetta, M., Miezin, F., Dobmeyer, S., Shulman, G., Petersen, S., 1990. Attentional modulation of neural processing of shape, color, and velocity in humans. Science (80-. ). 248, 1556-1559. https://doi.org/10.1126/SCIENCE.2360050

Corbetta, M., Shulman, G.L., 2002. Control of goal-directed and stimulus-driven attention in the brain. Nat. Rev. Neurosci. 3, 201-215. https://doi.org/10.1038/nrn755

Cornelio, P., Velasco, C., Obrist, M., 2021. Multisensory Integration as per Technological Advances: A Review. Front. Neurosci. https://doi.org/10.3389/fnins.2021.652611

Cox, S.M.L., Andrade, A., Johnsrude, I.S., 2005. Learning to like: A role for human orbitofrontal cortex in conditioned reward. J. Neurosci. 25, 2733-2740. https://doi.org/10.1523/JNEUROSCI.3360-04.2005

Dawkins, R., 2016. The selfish gene.

Della Libera, C., Chelazzi, L., 2009. Learning to Attend and to Ignore Is a Matter of Gains and Losses. Psychol. Sci. 20, 778-784. https://doi.org/10.1111/j.1467-9280.2009.02360.x

Della Libera, C., Chelazzi, L., 2006. Visual Selective Attention and the Effects of Monetary Rewards. Psychol. Sci. 17, 222-227. https://doi.org/10.1111/j.1467-9280.2006.01689.x

Delorme, A., Makeig, S., 2004. EEGLAB: An open source toolbox for analysis of single-trial EEG dynamics including independent component analysis. J. Neurosci. Methods. https://doi.org/10.1016/j.jneumeth.2003.10.009

Desimone, R., Duncan, J., 1995. NEURAL MECHANISMS OF SELECTIVE VISUAL ATTENTION, Annu. Rev. Neurosci.

Dickinson, A., Balleine, B., 1994. Motivational control of goal-directed action. Anim. Learn. Behav. 1994221 22, 1-18. https://doi.org/10.3758/BF03199951

Diederich, A., Colonius, H., 1987. Intersensory facilitation in the motor component? - A reaction time analysis. Psychol. Res. 49, 23-29. https://doi.org/10.1007/BF00309199

Driver, J., Noesselt, T., 2008. Multisensory Interplay Reveals Crossmodal Influences on "SensorySpecific" Brain Regions, Neural Responses, and Judgments. Neuron. https://doi.org/10.1016/j.neuron.2007.12.013

Duncan, J., 1984. Selective attention and the organization of visual information. J. Exp. Psychol. Gen. 113, 501-517. https://doi.org/10.1037/0096-3445.113.4.501

Duncan, J., Martens, S., Ward, R., 1997. Restricted attentional capacity within but not between sensory modalities. Nature 387, 808-810. https://doi.org/10.1038/42947

Dundon, N.M., Bertini, C., Làdavas, E., Sabel, B.A., Gall, C., 2015. Visual rehabilitation: Visual scanning, multisensory stimulation and vision restoration trainings. Front. Behav. Neurosci. https://doi.org/10.3389/fnbeh.2015.00192

Engelmann, J.B., Damaraju, E., Padmala, S., Pessoa, L., 2009. Combined effects of attention and motivation on visual task performance: Transient and sustained motivational effects. Front. Hum. Neurosci. 3, 4. https://doi.org/10.3389/neuro.09.004.2009

Eriksen, B.A., Eriksen, C.W., 1974. Effects of noise letters upon the identification of a target letter 
in a nonsearch task. Percept. Psychophys. 1974 161 16, 143-149. https://doi.org/10.3758/BF03203267

Etzel, J.A., Cole, M.W., Zacks, J.M., Kay, K.N., Braver, T.S., 2016. Reward Motivation Enhances Task Coding in Frontoparietal Cortex. Cereb. Cortex 26, 1647-1659. https://doi.org/10.1093/cercor/bhu327

Failing, M., Nissens, T., Pearson, D., Le Pelley, M., Theeuwes, J., 2015. Oculomotor capture by stimuli that signal the availability of reward. J. Neurophysiol. 114, 2316-2327. https://doi.org/10.1152/jn.00441.2015

Failing, M., Theeuwes, J., 2018. Selection history: How reward modulates selectivity of visual attention. Psychon. Bull. Rev. 25, 514-538. https://doi.org/10.3758/s13423-017-1380-y

Failing, M., Theeuwes, J., 2017. Don't let it distract you: how information about the availability of reward affects attentional selection. Attention, Perception, Psychophys. 79, 2275-2298. https://doi.org/10.3758/s13414-017-1376-8

Failing, M.F., Theeuwes, J., 2015. Nonspatial attentional capture by previously rewarded scene semantics. Vis. cogn. 23. https://doi.org/10.1080/13506285.2014.990546

Faul, F., Erdfelder, E., Lang, A.G., Buchner, A., 2007. G*Power 3: A flexible statistical power analysis program for the social, behavioral, and biomedical sciences, in: Behavior Research Methods. Psychonomic Society Inc., pp. 175-191. https://doi.org/10.3758/BF03193146

Feldmann-Wüstefeld, T., Schubö, A., 2013. Context homogeneity facilitates both distractor inhibition and target enhancement. J. Vis. 13. https://doi.org/10.1167/13.3.11

Feng, W., Stormer, V.S., Martinez, A., McDonald, J.J., Hillyard, S.A., 2014. Sounds Activate Visual Cortex and Improve Visual Discrimination. J. Neurosci. 34, 9817-9824. https://doi.org/10.1523/JNEUROSCI.4869-13.2014

Floresco, S.B., Magyar, O., 2006. Mesocortical dopamine modulation of executive functions: Beyond working memory. Psychopharmacology (Berl). https://doi.org/10.1007/s00213-0060404-5

Fox, C.R., Poldrack, R.A., 2009. Prospect theory and the brain, in: Neuroeconomics. Elsevier Inc., pp. 145-173. https://doi.org/10.1016/B978-0-12-374176-9.00011-7

Franzen, L., Delis, I., De Sousa, G., Kayser, C., Philiastides, M.G., 2020. Auditory information enhances post-sensory visual evidence during rapid multisensory decision-making. Nat. Commun. 11, 1-14. https://doi.org/10.1038/s41467-020-19306-7

Frassinetti, F., Bolognini, N., Làdavas, E., 2002. Enhancement of visual perception by crossmodal visuo-auditory interaction. Exp. Brain Res. 147, 332-343. https://doi.org/10.1007/s00221-002$1262-\mathrm{y}$

Frederick, S., Loewenstein, G., O’Donoghue, T., 2002. Time Discounting and Time Preference: A Critical Review. J. Econ. Lit. 40, 351-401. https://doi.org/10.1257/002205102320161311

Frens, M.A., Van Opstal, A.J., Van Der Willigen, R.F., 1995. Spatial and temporal factors determine auditory-visual interactions in human saccadic eye movements. Percept. Psychophys. 57, 802816. https://doi.org/10.3758/BF03206796

Friston, K., 2010. The free-energy principle: a unified brain theory? Nat. Rev. Neurosci. 2010112 11, 127-138. https://doi.org/10.1038/nrn2787

Garcia-Lazaro, H.G., Bartsch, M. V., Boehler, C.N., Krebs, R.M., Donohue, S.E., Harris, J.A., Schoenfeld, M.A., Hopf, J.M., 2018. Dissociating reward- and attention-driven biasing of global feature-based selection in human visual cortex. J. Cogn. Neurosci. 31, 469-481. https://doi.org/10.1162/jocn_a_01356

Giard, M.H., Peronnet, F., 1999. Auditory-visual integration during multimodal object recognition in humans: A behavioral and electrophysiological study. J. Cogn. Neurosci. 11, 473-490. https://doi.org/10.1162/089892999563544

Gold, J.I., Shadlen, M.N., 2007. The Neural Basis of Decision Making. Annu. Rev. Neurosci. 30, 535-574. https://doi.org/10.1146/annurev.neuro.29.051605.113038

Goldring, J.E., Dorris, M.C., Corneil, B.D., Ballantyne, P.A., Munoz, D.P., 1996. Combined eyehead gaze shifts to visual and auditory targets in humans. Exp. Brain Res. 111, 68-78. https://doi.org/10.1007/BF00229557

Goldstein, R.Z., Cottone, L.A., Jia, Z., Maloney, T., Volkow, N.D., Squires, N.K., 2006. The effect of graded monetary reward on cognitive event-related potentials and behavior in young healthy adults. Int. J. Psychophysiol. 62, 272-279. https://doi.org/10.1016/j.ijpsycho.2006.05.006 
Goltstein, P.M., Coffey, E.B.J., Roelfsema, P.R., Pennartz, C.M.A., 2013. In vivo two-photon Ca2+ imaging reveals selective reward effects on stimulus-specific assemblies in mouse visual cortex. J. Neurosci. 33, 11540-11555. https://doi.org/10.1523/JNEUROSCI.1341-12.2013

Goltstein, P.M., Meijer, G.T., Pennartz, C.M.A., 2018. Conditioning sharpens the spatial representation of rewarded stimuli in mouse primary visual cortex. Elife 7. https://doi.org/10.7554/eLife.37683

Gong, M., Yang, F., Li, S., 2016. Reward association facilitates distractor suppression in human visual search. Eur. J. Neurosci. 43, 942-953. https://doi.org/10.1111/ejn.13174

Grabenhorst, F., Rolls, E.T., Margot, C., 2011. A hedonically complex odor mixture produces an attentional capture effect in the brain. Neuroimage 55, 832-843. https://doi.org/10.1016/j.neuroimage.2010.12.023

Grégoire, L., Anderson, B.A., 2019. Semantic generalization of value-based attentional priority. Learn. Mem. 26, 460-464. https://doi.org/10.1101/lm.050336.119

Haber, S.N., 2011. Neuroanatomy of Reward: A View from the Ventral Striatum, Neurobiology of Sensation and Reward. CRC Press/Taylor \& Francis.

Halverson, H.M., 1927. The Upper Limit of Auditory Localization. Am. J. Psychol. 38, 97. https://doi.org/10.2307/1414648

Hammerschmidt, W., Kagan, I., Kulke, L., Schacht, A., 2018. Implicit reward associations impact face processing: Time-resolved evidence from event-related brain potentials and pupil dilations. Neuroimage 179, 557-569. https://doi.org/10.1016/j.neuroimage.2018.06.055

Henschke, J.U., Dylda, E., Katsanevaki, D., Dupuy, N., Currie, S.P., Amvrosiadis, T., Pakan, J.M.P., Rochefort, N.L., 2020. Reward Association Enhances Stimulus-Specific Representations in Primary Visual Cortex. Curr. Biol. 30, 1866-1880.e5. https://doi.org/10.1016/j.cub.2020.03.018

Herd, S., Krueger, K., Nair, A., Mollick, J., O'Reilly, R., 2021. Neural Mechanisms of Human Decision-Making. Cogn. Affect. Behav. Neurosci. 21, 35-57. https://doi.org/10.3758/s13415020-00842-0

Hickey, C., Chelazzi, L., Theeuwes, J., 2010a. Reward guides vision when it's your thing: Trait reward-seeking in reward-mediated visual priming. PLoS One 5, 14087. https://doi.org/10.1371/journal.pone.0014087

Hickey, C., Chelazzi, L., Theeuwes, J., 2010b. Reward changes salience in human vision via the anterior cingulate. J. Neurosci. 30, 11096-11103. https://doi.org/10.1523/JNEUROSCI.102610.2010

Hickey, C., Di Lollo, V., McDonald, J.J., 2009. Electrophysiological indices of target and distractor processing in visual search. J. Cogn. Neurosci. 21, 760-775. https://doi.org/10.1162/jocn.2009.21039

Hickey, C., Van Zoest, W., 2012. Reward creates oculomotor salience. Curr. Biol. https://doi.org/10.1016/j.cub.2012.02.007

Hoffman, J.E., Nelson, B., 1981. Spatial selectivity in visual search. Percept. Psychophys. 1981303 30, 283-290. https://doi.org/10.3758/BF03214284

Hollerman, J.R., Schultz, W., 1998. Dopamine neurons report an error in the temporal prediction of reward during learning. Nat. Neurosci. 1, 304-309. https://doi.org/10.1038/1124

Hopf, J.M., Boehler, C.N., Luck, S.J., Tsotsos, J.K., Heinze, H.J., Schoenfeld, M.A., 2006. Direct neurophysiological evidence for spatial suppression surrounding the focus of attention in vision. Proc. Natl. Acad. Sci. U. S. A. 103, 1053-1058. https://doi.org/10.1073/pnas.0507746103

Hughes, G., Mathan, S., Yeung, N., 2013. EEG indices of reward motivation and target detectability in a rapid visual detection task. Neuroimage 64, 590-600. https://doi.org/10.1016/j.neuroimage.2012.09.003

Hull, C., 1943. Principles of behavior: An introduction to behavior theory. Appleton-Century-Crofts, New York.

Itthipuripat, S., Vo, V.A., Sprague, T.C., Serences, J.T., 2019. Value-driven attentional capture enhances distractor representations in early visual cortex. PLoS Biol. 17, e3000186. https://doi.org/10.1371/journal.pbio.3000186

Itti, L., Koch, C., 2001. Computational modelling of visual attention. Nat. Rev. Neurosci. 2, 194203. https://doi.org/10.1038/35058500 
Jay, T.M., 2003. Dopamine: A potential substrate for synaptic plasticity and memory mechanisms. Prog. Neurobiol. https://doi.org/10.1016/S0301-0082(03)00085-6

Kahneman, D., Treisman, A., Gibbs, B.J., 1992. The reviewing of object files: Object-specific integration of information. Cogn. Psychol. 24, 175-219. https://doi.org/10.1016/00100285(92)90007-O

Kahneman, D., Tversky, A., 2013. Choices, Values, and Frames. pp. 269-278. https://doi.org/10.1142/9789814417358_0016

Kang, G., Chang, W., Wang, L., Wei, P., Zhou, X., 2018. Reward enhances cross-modal conflict control in object categorization: Electrophysiological evidence. Psychophysiology 55, e13214. https://doi.org/10.1111/psyp.13214

Kang, G., Wang, L., Zhou, X., 2017. Reward interacts with modality shift to reduce cross-modal conflict. J. Vis. 17. https://doi.org/10.1167/17.1.19

Keitel, C., Maess, B., Schröger, E., Müller, M.M., 2013. Early visual and auditory processing rely on modality-specific attentional resources. Neuroimage 70, 240-249. https://doi.org/10.1016/j.neuroimage.2012.12.046

Kim, A.J., Lee, D.S., Anderson, B.A., 2021. Previously reward-associated sounds interfere with goaldirected auditory processing. Q. J. Exp. Psychol. 74, 1257-1263. https://doi.org/10.1177/1747021821990033

Kiss, M., Driver, J., Eimer, M., 2009. Reward Priority of Visual Target Singletons Modulates EventRelated Potential Signatures of Attentional Selection. Psychol. Sci. 20, 245-251. https://doi.org/10.1111/j.1467-9280.2009.02281.x

Klingel, M., Kopčo, N., Laback, B., 2021. Reweighting of Binaural Localization Cues Induced by Lateralization Training. JARO - J. Assoc. Res. Otolaryngol. 1-16. https://doi.org/10.1007/s10162-021-00800-8

Krebs, R.M., Boehler, C.N., Appelbaum, L.G., Woldorff, M.G., 2013. Reward Associations Reduce Behavioral Interference by Changing the Temporal Dynamics of Conflict Processing. PLoS One 8. https://doi.org/10.1371/journal.pone.0053894

Krebs, R.M., Boehler, C.N., Egner, T., Woldorff, M.G., 2011. The neural underpinnings of how reward associations can both guide and misguide attention. J. Neurosci. 31, 9752-9759. https://doi.org/10.1523/JNEUROSCI.0732-11.2011

Krebs, R.M., Boehler, C.N., Woldorff, M.G., 2010. The influence of reward associations on conflict processing in the Stroop task. Cognition 117, 341-347. https://doi.org/10.1016/j.cognition.2010.08.018

Kristjánsson, Á., Sigurjónsdóttir, Ó., Driver, J., 2010. Fortune and reversals of fortune in visual search: Reward contingencies for pop-out targets affect search efficiency and target repetition effects. Attention, Perception, Psychophys. 72, 1229-1236. https://doi.org/10.3758/APP.72.5.1229

Làdavas, E., Tosatto, L., Bertini, C., 2020. Behavioural and functional changes in neglect after multisensory stimulation. Neuropsychol. Rehabil. https://doi.org/10.1080/09602011.2020.1786411

Lakens, D., 2013. Calculating and reporting effect sizes to facilitate cumulative science: a practical primer for t-tests and ANOVAs. Front. Psychol. 4, 863. https://doi.org/10.3389/fpsyg.2013.00863

Le Pelley, M.E., Mitchell, C.J., Beesley, T., George, D.N., Wills, A.J., 2016. Attention and associative learning in humans: An integrative review. Psychol. Bull. 142, 1111-1140. https://doi.org/10.1037/bul0000064

Leo, F., Noppeney, U., 2014. Conditioned Sounds Enhance Visual Processing. PLoS One 9, e106860. https://doi.org/10.1371/journal.pone.0106860

Leo, F., Romei, V., Freeman, E., Ladavas, E., Driver, J., 2011. Looming sounds enhance orientation sensitivity for visual stimuli on the same side as such sounds. Exp. Brain Res. 213, 193-201. https://doi.org/10.1007/s00221-011-2742-8

Liu, D., Deng, J., Zhang, Z., Zhang, Z.Y., Sun, Y.G., Yang, T., Yao, H., 2020. Orbitofrontal control of visual cortex gain promotes visual associative learning. Nat. Commun. 11, 1-14. https://doi.org/10.1038/s41467-020-16609-7

Liu, J., Zhang, C., Zhu, Y., Liu, Y., Sun, H., Ristaniemi, T., Cong, F., Parviainen, T., 2020. Dissociable Effects of Reward on P300 and EEG Spectra Under Conditions of High vs. Low 
Vigilance During a Selective Visual Attention Task. Front. Hum. Neurosci. 14, 207. https://doi.org/10.3389/fnhum.2020.00207

Lockhofen, D.E.L., Mulert, C., 2021. Neurochemistry of Visual Attention. Front. Neurosci. 15, 517. https://doi.org/10.3389/fnins.2021.643597

Luck, S.J., Hillyard, S.A., Mouloua, M., Woldorff, M.G., et al, 1994. Effects of spatial cuing on luminance detectability: Psychophysical and electrophysiological evidence for early selection. J. Exp. Psychol. Hum. Percept. Perform. 20, 887-904. https://doi.org/10.1037//00961523.20.4.887

Luque, D., Beesley, T., Morris, R.W., Jack, B.N., Griffiths, O., Whitford, T.J., Le Pelley, M.E., 2017. Goal-directed and habit-like modulations of stimulus processing during reinforcement learning. J. Neurosci. 37, 3009-3017. https://doi.org/10.1523/JNEUROSCI.3205-16.2017

Luque, D., Morís, J., Rushby, J.A., Le Pelley, M.E., 2015. Goal-directed EEG activity evoked by discriminative stimuli in reinforcement learning. Psychophysiology 52, 238-248. https://doi.org/10.1111/psyp.12302

Maclean, M.H., Giesbrecht, B., 2015. Neural evidence reveals the rapid effects of reward history on selective attention. Brain Res. 1606, 86-94. https://doi.org/10.1016/j.brainres.2015.02.016

Macmillan, N.A., Creelman, C.D., 1991. Detection theory: A user's guide., Detection theory: A user's guide. Cambridge University Press, New York, NY, US.

Maier, M., Ballester, B.R., Verschure, P.F.M.J., 2019. Principles of Neurorehabilitation After Stroke Based on Motor Learning and Brain Plasticity Mechanisms. Front. Syst. Neurosci. https://doi.org/10.3389/fnsys.2019.00074

Mangun, G.R., 1995. Neural mechanisms of visual selective attention. Psychophysiology 32, 4-18. https://doi.org/10.1111/j.1469-8986.1995.tb03400.x

Manohar, S.G., Chong, T.T.J., Apps, M.A.J., Batla, A., Stamelou, M., Jarman, P.R., Bhatia, K.P., Husain, M., 2015. Reward Pays the Cost of Noise Reduction in Motor and Cognitive Control. Curr. Biol. 25, 1707-1716. https://doi.org/10.1016/j.cub.2015.05.038

Manohar, S.G., Muhammed, K., Fallon, S.J., Husain, M., 2019. Motivation dynamically increases noise resistance by internal feedback during movement. Neuropsychologia 123, 19-29. https://doi.org/10.1016/j.neuropsychologia.2018.07.011

Maunsell, J.H.R., 2004. Neuronal representations of cognitive state: Reward or attention? Trends Cogn. Sci. 8, 261-265. https://doi.org/10.1016/j.tics.2004.04.003

McDonald, J.J., Störmer, V.S., Martinez, A., Feng, W., Hillyard, S.A., 2013. Salient Sounds Activate Human Visual Cortex Automatically. J. Neurosci. 33, 9194.

Meredith, M.A., Stein, B.E., 1986. Visual, auditory, and somatosensory convergence on cells in superior colliculus results in multisensory integration. J. Neurophysiol. 56, 640-662. https://doi.org/10.1152/jn.1986.56.3.640

Mine, C., Saiki, J., 2015. Task-irrelevant stimulus-reward association induces value-driven attentional capture. Attention, Perception, Psychophys. 77, 1896-1907. https://doi.org/10.3758/s13414-015-0894-5

Mogami, T., Tanaka, K., 2006. Reward association affects neuronal responses to visual stimuli in macaque TE and perirhinal cortices. J. Neurosci. 26, 6761-6770. https://doi.org/10.1523/JNEUROSCI.4924-05.2006

Mognon, A., Jovicich, J., Bruzzone, L., Buiatti, M., 1994. ADJUST: An automatic EEG artifact detector based on the joint use of spatial and temporal features. Psychophysiology. https://doi.org/10.1111/j.1469-8986.2010.01061.x

Molholm, S., Ritter, W., Murray, M.M., Javitt, D.C., Schroeder, C.E., Foxe, J.J., 2002. Multisensory auditory-visual interactions during early sensory processing in humans: A high-density electrical mapping study, in: Cognitive Brain Research. Elsevier, pp. 115-128. https://doi.org/10.1016/S0926-6410(02)00066-6

Motter, B.C., 1993. Focal attention produces spatially selective processing in visual cortical areas V1, V2, and V4 in the presence of competing stimuli. J. Neurophysiol. 70, 909-919. https://doi.org/10.1152/jn.1993.70.3.909

Mounts, J.R.W., 2000. Attentional capture by abrupt onsets and feature singletons produces inhibitory surrounds. Percept. Psychophys. 62, 1485-1493. https://doi.org/10.3758/BF03212148

Nakayama, K., 1990. The iconic bottleneck and the tenuous link between early visual processing and 
perception, in: Blakemore, C. (Ed.), Vision: Coding and Efficiency. Cambridge: Cambridge University Press, pp. 411-422.

Navarra, J., Alsius, A., Soto-Faraco, S., Spence, C., 2010. Assessing the role of attention in the audiovisual integration of speech. Inf. Fusion 11, 4-11. https://doi.org/10.1016/j.inffus.2009.04.001

Ngo, M.K., Spence, C., 2010. Crossmodal facilitation of masked visual target identification. Attention, Perception, Psychophys. 72, 1938-1947. https://doi.org/10.3758/APP.72.7.1938

Niv, Y., Joel, D., Dayan, P., 2006. A normative perspective on motivation. Trends Cogn. Sci. 10, 375-381. https://doi.org/10.1016/J.TICS.2006.06.010

Nobre, A.C., Rao, A., Chelazzi, L., 2006. Selective attention to specific features within objects: Behavioral and electrophysiological evidence. J. Cogn. Neurosci. 18, 539-561. https://doi.org/10.1162/jocn.2006.18.4.539

Novak, K.D., Foti, D., 2015. Teasing apart the anticipatory and consummatory processing of monetary incentives: An event-related potential study of reward dynamics. Psychophysiology 52, 1470-1482. https://doi.org/10.1111/psyp.12504

O'Doherty, J.P., Cockburn, J., Pauli, W.M., 2017. Learning, Reward, and Decision Making. Annu. Rev. Psychol. 68, 73-100. https://doi.org/10.1146/annurev-psych-010416-044216

Otto, A.R., Daw, N.D., 2019. The opportunity cost of time modulates cognitive effort. Neuropsychologia 123, 92-105. https://doi.org/10.1016/j.neuropsychologia.2018.05.006

Padmala, S., Pessoa, L., 2010. Interactions between cognition and motivation during response inhibition. Neuropsychologia https://doi.org/10.1016/j.neuropsychologia.2009.10.017

Pavlov, 1927. Conditioned Reflexes. Oxford University Press, Oxford.

Pearson, D., Watson, P., Cheng, P.X., Le Pelley, M.E., 2020. Overt Attentional Capture by RewardRelated Stimuli Overcomes Inhibitory Suppression. J. Exp. Psychol. Hum. Percept. Perform. https://doi.org/10.1037/xhp0000728

Pessiglione, M., Schmidt, L., Draganski, B., Kalisch, R., Lau, H., Dolan, R.J., Frith, C.D., 2007. How the Brain Translates Money into Force: A Neuroimaging Study of Subliminal Motivation. Science 316, 904. https://doi.org/10.1126/SCIENCE.1140459

Pessiglione, M., Seymour, B., Flandin, G., Dolan, R.J., Frith, C.D., 2006. Dopamine-dependent prediction errors underpin reward-seeking behaviour in humans. Nature 442, 1042-1045. https://doi.org/10.1038/nature05051

Pessiglione, M., Vinckier, F., Bouret, S., Daunizeau, J., Le Bouc, R., 2018. Why not try harder? Computational approach to motivation deficits in neuro-psychiatric diseases, Brain.

Pessoa, L., 2015. Multiple influences of reward on perception and attention. Vis. cogn. 23, 272-290. https://doi.org/10.1080/13506285.2014.974729

Pessoa, L., 2009. How do emotion and motivation direct executive control? Trends Cogn. Sci. 13, 160-166. https://doi.org/10.1016/j.tics.2009.01.006

Pessoa, L., Engelmann, J.B., 2010. Embedding reward signals into perception and cognition. Front. Neurosci. 4, 17. https://doi.org/10.3389/fnins.2010.00017

Peters, J., Büchel, C., 2010. Neural representations of subjective reward value. Behav. Brain Res. 213, 135-141. https://doi.org/10.1016/J.BBR.2010.04.031

Pfabigan, D.M., Seidel, E.M., Sladky, R., Hahn, A., Paul, K., Grahl, A., Küblböck, M., Kraus, C., Hummer, A., Kranz, G.S., Windischberger, C., Lanzenberger, R., Lamm, C., 2014. P300 amplitude variation is related to ventral striatum BOLD response during gain and loss anticipation: An EEG and fMRI experiment. Neuroimage 96, 12-21. https://doi.org/10.1016/j.neuroimage.2014.03.077

Pick, H.L., Warren, D.H., Hay, J.C., 1969. Sensory conflict in judgments of spatial direction. Percept. Psychophys. 6, 203-205. https://doi.org/10.3758/BF03207017

Platt, M.L., Glimcher, P.W., 1999. Neural correlates of decision variables in parietal cortex. Nature 400, 233-238. https://doi.org/10.1038/22268

Pleger, B., Blankenburg, F., Ruff, C.C., Driver, J., Dolan, R.J., 2008. Reward facilitates tactile judgments and modulates hemodynamic responses in human primary somatosensory cortex. J. Neurosci. 28, 8161-8168. https://doi.org/10.1523/JNEUROSCI.1093-08.2008

Pool, E., Brosch, T., Delplanque, S., Sander, D., 2014. Where is the chocolate? Rapid spatial orienting toward stimuli associated with primary rewards. Cognition 130, 348-359. 
https://doi.org/10.1016/j.cognition.2013.12.002

Pooresmaeili, A., FitzGerald, T.H.B., Bach, D.R., Toelch, U., Ostendorf, F., Dolan, R.J., 2014. Cross-modal effects of value on perceptual acuity and stimulus encoding. Proc. Natl. Acad. Sci. U. S. A. 111, 15244-15249. https://doi.org/10.1073/pnas.1408873111

Pooresmaeili, A., Roelfsema, P.R., 2014. A growth-cone model for the spread of object-based attention during contour grouping. Curr. Biol. 24. https://doi.org/10.1016/j.cub.2014.10.007

Pornpattananangkul, N., Nusslock, R., 2015. Motivated to win: Relationship between anticipatory and outcome reward-related neural activity. Brain Cogn. 100, 21-40. https://doi.org/10.1016/j.bandc.2015.09.002

Posner, M.I., 1980. Orienting of Attention. Q. J. Exp. Psychol. 32, 3-25. https://doi.org/10.1080/00335558008248231

Qin, N., Gu, R., Xue, J., Chen, C., Zhang, M., 2021. Reward-driven attention alters perceived salience. J. Vis. 21, 1-13. https://doi.org/10.1167/JOV.21.1.7

Rangel, A., Camerer, C., Montague, P.R., 2008. A framework for studying the neurobiology of valuebased decision making. Nat. Rev. Neurosci. https://doi.org/10.1038/nrn2357

Raymond, J.E., O'Brien, J.L., 2009. Selective visual attention and motivation: the consequences of value learning in an attentional blink task. Psychol. Sci. 20, 981-8. https://doi.org/10.1111/j.1467-9280.2009.02391.x

Reynolds, J.H., Chelazzi, L., 2004. Attentional modulation of visual processing. Annu. Rev. Neurosci. https://doi.org/10.1146/annurev.neuro.26.041002.131039

Reynolds, J.H., Chelazzi, L., Desimone, R., 1999. Competitive mechanisms subserve attention in macaque areas V2 and V4. J. Neurosci. 19, 1736-1753. https://doi.org/10.1523/jneurosci.1905-01736.1999

Rock, I., Gutman, D., 1981. The effect of inattention on form perception. J. Exp. Psychol. Hum. Percept. Perform. 7, 275-285. https://doi.org/10.1037/0096-1523.7.2.275

Rolls, E.T., 2015. Taste, olfactory, and food reward value processing in the brain. Prog. Neurobiol. https://doi.org/10.1016/j.pneurobio.2015.03.002

Rossi, V., Vanlessen, N., Bayer, M., Grass, A., Pourtois, G., Schacht, A., 2017. Motivational salience modulates early visual cortex responses across task sets. J. Cogn. Neurosci. 29, 968-979. https://doi.org/10.1162/jocn_a_01093

Rusz, D., Bijleveld, E., Kompier, M.A.J., 2018. Reward-associated distractors can harm cognitive performance. PLoS One 13. https://doi.org/10.1371/journal.pone.0205091

Rusz, D., Le Pelley, M.E., Kompier, M.A.J., Mait, L., Bijleveld, E., 2020. Reward-Driven distraction: A meta-analysis. Psychol. Bull. 146, 872-899.

Rutherford, H.J.V., O'Brien, J.L., Raymond, J.E., 2010. Value associations of irrelevant stimuli modify rapid visual orienting. Psychon. Bull. Rev. 17, 536-542. https://doi.org/10.3758/PBR.17.4.536

Rutkowski, R.G., Weinberger, N.M., 2005. Encoding of learned importance of sound by magnitude of representational area in primary auditory cortex. Proc. Natl. Acad. Sci. U. S. A. 102, 1366413669. https://doi.org/10.1073/pnas.0506838102

San Martín, R., Appelbaum, L.G., Huettel, S.A., Woldorff, M.G., 2016. Cortical Brain Activity Reflecting Attentional Biasing Toward Reward-Predicting Cues Covaries with Economic Decision-Making Performance. Cereb. Cortex 26, 1-11. https://doi.org/10.1093/cercor/bhu160

Sanz, L.R.D., Vuilleumier, P., Bourgeois, A., 2018. Cross-modal integration during value-driven attentional capture. $\quad$ Neuropsychologia $120, \quad 105-112$. https://doi.org/10.1016/j.neuropsychologia.2018.10.014

Sawaki, R., Luck, S.J., Raymond, J.E., 2015. How attention changes in response to incentives. J. Cogn. Neurosci. 27, 2229-2239. https://doi.org/10.1162/jocn_a_00847

Schacht, A., Adler, N., Chen, P., Guo, T., Sommer, W., 2012. Association with positive outcome induces early effects in event-related brain potentials. Biol. Psychol. 89, 130-136. https://doi.org/10.1016/j.biopsycho.2011.10.001

Schultz, W., 2015. Neuronal reward and decision signals: From theories to data. Physiol. Rev. 95, 853-951. https://doi.org/10.1152/physrev.00023.2014

Schultz, W., 2013. Updating dopamine reward signals. Curr. Opin. Neurobiol. 23, 229-238. https://doi.org/10.1016/J.CONB.2012.11.012

Schultz, W., 2006. Behavioral Theories and the Neurophysiology of Reward. Annu. Rev. Psychol. 
57, 87-115. https://doi.org/10.1146/annurev.psych.56.091103.070229

Schultz, W., 2002. Getting Formal with Dopamine and Reward. Neuron 36, 241-263. https://doi.org/10.1016/S0896-6273(02)00967-4

Schultz, W., 2000. Multiple reward signals in the brain 1, 199-207. https://doi.org/10.1038/35044563

Schultz, W., 1998. Predictive Reward Signal of Dopamine Neurons. J. Neurophysiol. 80, 1-27. https://doi.org/10.1152/jn.1998.80.1.1

Schultz, W., 1986. Responses of midbrain dopamine neurons to behavioral trigger stimuli in the monkey. J. Neurophysiol. 56, 1439-1461. https://doi.org/10.1152/jn.1986.56.5.1439

Schultz, W., Dayan, P., Montague, P.R., 1997. A Neural Substrate of Prediction and Reward. Science (80-. ). 275, 1593-1599. https://doi.org/10.1126/science.275.5306.1593

Seitz, A.R., Kim, R., Shams, L., 2006. Sound Facilitates Visual Learning. Curr. Biol. 16, 1422-1427. https://doi.org/10.1016/j.cub.2006.05.048

Serences, J.T., 2008. Value-Based Modulations in Human Visual Cortex. Neuron 60, 1169-1181. https://doi.org/10.1016/j.neuron.2008.10.051

Serences, J.T., Saproo, S., 2010. Population response profiles in early visual cortex are biased in favor of more valuable stimuli. J. Neurophysiol. 104, 76-87. https://doi.org/10.1152/jn.01090.2009

Serences, J.T., Saproo, S., Scolari, M., Ho, T., Muftuler, L.T., 2009. Estimating the influence of attention on population codes in human visual cortex using voxel-based tuning functions. Neuroimage 44, 223-231. https://doi.org/10.1016/J.NEUROIMAGE.2008.07.043

Shadmehr, R., Xivry, J.J.O. de, Xu-Wilson, M., Shih, T.-Y., 2010. Temporal Discounting of Reward and the Cost of Time in Motor Control. J. Neurosci. 30, 10507-10516. https://doi.org/10.1523/JNEUROSCI.1343-10.2010

Shams, L., Kamitani, Y., Shimojo, S., 2000. Illusions: What you see is what you hear. Nature 408, 788. https://doi.org/10.1038/35048669

Shams, L., Seitz, A.R., 2008. Benefits of multisensory learning. Trends Cogn. Sci. 12, 411-417. https://doi.org/10.1016/j.tics.2008.07.006

Shapcott, K.A., Schmiedt, J.T., Kouroupaki, K., Kienitz, R., Lazar, A., Singer, W., Schmid, M.C., 2020. Reward-related suppression of neural activity in macaque visual area v4. Cereb. Cortex 30, 4871-4881. https://doi.org/10.1093/cercor/bhaa079

Shuler, M.G., Bear, M.F., 2006. Reward timing in the primary visual cortex. Science (80-. ). 311, 1606-1609. https://doi.org/10.1126/science.1123513

Slutsky, D.A., Recanzone, G.H., 2001. Temporal and spatial dependency of the ventriloquism effect. Neuroreport 12, 7-10. https://doi.org/10.1097/00001756-200101220-00009

Sobal, J., 1998. Cultural Comparison Research Designs in Food, Eating, and Nutrition. Food Qual. Prefer. 9, 385-392. https://doi.org/10.1016/S0950-3293(98)00029-9

Stanisor, L., Van Der Togt, C., Pennartz, C.M.A., Roelfsema, P.R., 2013. A unified selection signal for attention and reward in primary visual cortex. Proc. Natl. Acad. Sci. U. S. A. 110, 91369141. https://doi.org/10.1073/pnas.1300117110

Stein, B.E., Meredith, M.A., 1993. The merging of the senses. The MIT Press.

Steinberg, E.E., Keiflin, R., Boivin, J.R., Witten, I.B., Deisseroth, K., Janak, P.H., 2013. A causal link between prediction errors, dopamine neurons and learning. Nat. Neurosci. 16, 966-973. https://doi.org/10.1038/nn.3413

Stine, G.M., Zylberberg, A., Ditterich, J., Shadlen, M.N., 2020. Differentiating between integration and non-integration strategies in perceptual decision making. Elife 9. https://doi.org/10.7554/eLife.55365

Störmer, V.S., Alvarez, G.A., 2014. Feature-based attention elicits surround suppression in feature space. Curr. Biol. 24, 1985-1988. https://doi.org/10.1016/j.cub.2014.07.030

Störmer, V.S., McDonald, J.J., Hillyard, S.A., 2009. Cross-modal cueing of attention alters appearance and early cortical processing of visual stimuli. Proc. Natl. Acad. Sci. U. S. A. 106, 22456-22461. https://doi.org/10.1073/pnas.0907573106

Sutton, R.S., Barto, A.G., 2018. Reinforcement Learning: An Introduction. MIT Press, Cambridge; MA.

Takakuwa, N., Kato, R., Redgrave, P., Isa, T., 2017. Emergence of visually-evoked reward expectation signals in dopamine neurons via the superior colliculus in V1 lesioned monkeys. 
Elife 6. https://doi.org/10.7554/eLife.24459

Takikawa, Y., Kawagoe, R., Itoh, H., Nakahara, H., Hikosaka, O., 2002. Modulation of saccadic eye movements by predicted reward outcome. Exp. Brain Res. 142. https://doi.org/10.1007/s00221001-0928-1

Talsma, D., Doty, T.J., Woldorff, M.G., 2007. Selective attention and audiovisual integration: Is attending to both modalities a prerequisite for early integration? Cereb. Cortex 17, 679-690. https://doi.org/10.1093/cercor/bhk016

Talsma, D., Woldorff, M.G., 2005. Selective attention and multisensory integration: Multiple phases of effects on the evoked brain activity. J. Cogn. Neurosci. 17, 1098-1114. https://doi.org/10.1162/0898929054475172

Tankelevitch, L., Spaak, E., Rushworth, M., Stokes, M., 2019. Previously reward-associated stimuli capture spatial attention in the absence of changes in the corresponding sensory representations as measured with MEG. bioRxiv 622589. https://doi.org/10.1101/622589

Tankelevitch, L., Spaak, E., Rushworth, M.F.S., Stokes, M.G., 2020. Previously Reward-Associated Stimuli Capture Spatial Attention in the Absence of Changes in the Corresponding Sensory Representations as Measured with MEG. J. Neurosci. 40, 5033-5050. https://doi.org/10.1523/JNEUROSCI.1172-19.2020

Teder-Sälejärvi, W.A., Di Russo, F., McDonald, J.J., Hillyard, S.A., 2005. Effects of spatial congruity on audio-visual multimodal integration. J. Cogn. Neurosci. 17, 1396-1409. https://doi.org/10.1162/0898929054985383

Teder-Sälejärvi, W.A., McDonald, J.J., Di Russo, F., Hillyard, S.A., 2002. An analysis of audiovisual crossmodal integration by means of event-related potential (ERP) recordings, in: Cognitive Brain Research. https://doi.org/10.1016/S0926-6410(02)00065-4

Theeuwes, J., 2019. Goal-driven, stimulus-driven, and history-driven selection. Curr. Opin. Psychol. https://doi.org/10.1016/j.copsyc.2018.12.024

Theeuwes, J., 2018. Visual selection: Usually fast and automatic; Seldom slow and volitional. J. Cogn. 1. https://doi.org/10.5334/joc.13

Theeuwes, J., Belopolsky, A. V., 2012. Reward grabs the eye: Oculomotor capture by rewarding stimuli. Vision Res. 74, 80-85. https://doi.org/10.1016/j.visres.2012.07.024

Thorndike, E.L., 1911. Animal intelligence. Macmillan, New York.

Trappenberg, T.P., Dorris, M.C., Munoz, D.P., Klein, R.M., 2001. A model of saccade initiation based on the competitive integration of exogenous and endogenous signals in the superior colliculus. J. Cogn. Neurosci. 13, 256-271. https://doi.org/10.1162/089892901564306

Vakhrushev, R., Cheng, F., Schacht, A., Pooresmaeili, A., 2021. Differential effects of intra-modal and cross-modal reward value on visual perception: ERP evidence. bioRxiv 2021.09.29.462374. https://doi.org/10.1101/2021.09.29.462374

Van Den Berg, B., Krebs, R.M., Lorist, M.M., Woldorff, M.G., 2014. Utilization of reward-prospect enhances preparatory attention and reduces stimulus conflict. Cogn. Affect. Behav. Neurosci. 14, 561-577. https://doi.org/10.3758/s13415-014-0281-Z

Van Zoest, W., Donk, M., Theeuwes, J., 2004. The role of stimulus-driven and goal-driven control in saccadic visual selection. J. Exp. Psychol. Hum. Percept. Perform. 30, 746-759. https://doi.org/10.1037/0096-1523.30.4.749

Vitay, J., Hamker, F.H., 2007. On the role of dopamine in cognitive vision, in: Lecture Notes in Computer Science (Including Subseries Lecture Notes in Artificial Intelligence and Lecture Notes in Bioinformatics). Springer Verlag, pp. 352-366. https://doi.org/10.1007/978-3-54077343-6_23

Von Kriegstein, K., Giraud, A.L., 2006. Implicit multisensory associations influence voice recognition. PLoS Biol. 4, 1809-1820. https://doi.org/10.1371/journal.pbio.0040326

Wang, L., Yu, H., Zhou, X., 2013. Interaction between value and perceptual salience in value-driven attentional capture. J. Vis. 13, 5-5. https://doi.org/10.1167/13.3.5

Watson, A.B., Pelli, D.G., 1983. Quest: A Bayesian adaptive psychometric method. Percept. Psychophys. 33, 113-120. https://doi.org/10.3758/BF03202828

Watson, P., Pearson, D., Most, S.B., Theeuwes, J., Wiers, R.W., Le Pelley, M.E., 2019. Attentional capture by Pavlovian reward-signalling distractors in visual search persists when rewards are removed. PLoS One 14, e0226284. https://doi.org/10.1371/journal.pone.0226284

Watson, R., Latinus, M., Noguchi, T., Garrod, O., Crabbe, F., Belin, P., 2014. Crossmodal adaptation 
in right posterior superior temporal sulcus during face-voice emotional integration. J. Neurosci. 34, 6813-6821. https://doi.org/10.1523/JNEUROSCI.4478-13.2014

Weil, R.S., Furl, N., Ruff, C.C., Symmonds, M., Flandin, G., Dolan, R.J., Driver, J., Rees, G., 2010. Rewarding Feedback After Correct Visual Discriminations Has Both General and Specific Influences on Visual Cortex. J. Neurophysiol. 104, 1746-1757. https://doi.org/10.1152/jn.00870.2009

Weinberger, N.M., 2007. Associative representational plasticity in the auditory cortex: A synthesis of two disciplines. Learn. Mem. https://doi.org/10.1101/lm.421807

Welch, R.B., Warren, D.H., 1980. Immediate perceptual response to intersensory discrepancy. Psychol. Bull. 88, 638-667. https://doi.org/10.1037/0033-2909.88.3.638

Westbrook, A., Frank, M.J., Cools, R., 2021. A mosaic of cost-benefit control over cortico-striatal circuitry. Trends Cogn. Sci. https://doi.org/10.1016/j.tics.2021.04.007

Wikman, P., Rinne, T., Petkov, C.I., 2019. Reward cues readily direct monkeys' auditory performance resulting in broad auditory cortex modulation and interaction with sites along cholinergic and dopaminergic pathways. Sci. Rep. 9. https://doi.org/10.1038/s41598-01938833-y

Wild, B., Treue, S., 2021. Primate extrastriate cortical area MST: a gateway between sensation and cognition. J. Neurophysiol. 125, 1851-1882. https://doi.org/10.1152/jn.00384.2020

Wise, R.A., 2004. Dopamine, learning and motivation. Nat. Rev. Neurosci. https://doi.org/10.1038/nrn1406

Wittmann, M., Leland, D.S., Paulus, M.P., 2007. Time and decision making: differential contribution of the posterior insular cortex and the striatum during a delay discounting task. Exp. Brain Res. 20061794 179, 643-653. https://doi.org/10.1007/S00221-006-0822-Y

Woldorff, M.G., 1993. Distortion of ERP averages due to overlap from temporally adjacent ERPs: Analysis and correction. Psychophysiology 30, 98-119. https://doi.org/10.1111/j.14698986.1993.tb03209.x

Woldorff, M.G., Fox, P.T., Matzke, M., Lancaster, J.L., Veeraswamy, S., Zamarripa, F., Seabolt, M., Glass, T., Gao, J.H., Martin, C.C., Jerabek, P., 1997. Retinotopic organization of early visual spatial attention effects as revealed by PET and ERPs, in: Human Brain Mapping. Hum Brain Mapp, pp. 280-286. https://doi.org/10.1002/(SICI)1097-0193(1997)5:4<280::AIDHBM13>3.0.CO;2-I

Wolfe, J.M., 2021. Guided Search 6.0: An updated model of visual search. Psychon. Bull. Rev. 28, 1060-1092. https://doi.org/10.3758/s13423-020-01859-9

Woods, T.M., Recanzone, G.H., 2004. Cross-Modal Interactions Evidenced, in: The Handbook of Multisensory Processes. MIT Press, Cambridge, MA, pp. 35-48.

Yantis, S., Anderson, B.A., Wampler, E.K., Laurent, P.A., 2012. Reward and attentional control in visual search. Nebraska Symp. Motiv. https://doi.org/10.1007/978-1-4614-4794-8-5

Yeung, N., Sanfey, A.G., 2004. Independent coding of reward magnitude and valence in the human brain. J. Neurosci. 24, 6258-6264. https://doi.org/10.1523/JNEUROSCI.4537-03.2004

Zelinsky, G.J., Bisley, J.W., 2015. The what, where, and why of priority maps and their interactions with visual working memory. Ann. N. Y. Acad. Sci. 1339, 154-164. https://doi.org/10.1111/nyas.12606

Zimmer, U., Itthipanyanan, S., Grent-'T-Jong, T., Woldorff, M.G., 2010. The electrophysiological time course of the interaction of stimulus conflict and the multisensory spread of attention. Eur. J. Neurosci. 31, 1744-1754. https://doi.org/10.1111/j.1460-9568.2010.07229.x 


\section{Chapter 4: Interaction of spatial attention and associative reward value of audiovisual stimuli}

Roman Vakhrushev ${ }^{1}$, Arezoo Pooresmaeili ${ }^{1 *}$

${ }^{1}$ Perception and Cognition Lab, European Neuroscience Institute Goettingen- A Joint

Initiative of the University Medical Center Goettingen and the Max-Planck-Society,

Germany, Grisebachstrasse 5, 37077 Goettingen, Germany

*Corresponding author: a.pooresmaeili@eni-g.de 


\begin{abstract}
Reward value and selective attention both enhance the representation of sensory stimuli at the earliest stages of processing. It is still debated whether and how reward-driven and attentional mechanisms interact to influence perception. Here we ask whether the interaction between reward value and selective attention depends on the sensory modality through which reward information is conveyed. Human participants first learned the reward value of uni-modal visual and auditory cues during a conditioning phase. Subsequently, they performed a change detection task on bimodal audiovisual stimuli containing a previously rewarded stimulus in one, both, or neither of the modalities. Importantly, participants were required to focus their attention on one side and only report changes that occurred on the attended side. Our results showed a strong modulation of visual and auditory event-related potentials (ERPs) by spatial attention. We found no main effect of reward value but importantly an interaction effect was found as the strength of attentional modulation of the ERPs was significantly affected by the reward value. When reward effects were inspected separately with respect to each modality, auditory value-driven modulation of attention was found to dominate the ERP effects whereas visual reward value on its own led to no effect, likely due to its interference with the target processing. These results inspire a two-stage model where first the salience of a high reward stimulus is enhanced on a local priority map specific to each sensory modality, and at a second stage reward value and top-down attentional mechanisms are integrated across sensory modalities to affect perception.
\end{abstract}




\subsection{Introduction}

Our surrounding environment contains large amounts of information, whereas our brain's processing capacity is limited. An important mechanism to face this challenge is to prioritize the processing of information that is relevant to our current goals and is associated with the most valuable outcome. However, these aspects, i.e., relevance and reward value, may not be always in the same direction. For instance, when waiting for our friend to pick us up at an intersection, we try to focus our attention on the side where our friend is most likely to arrive. At the same time, we may also notice the sound or sight of approaching vehicles that deliver takeout food, and if hungry we may be even more sensitive to these sources information compared to the familiar sight of our friend's car. Due to the prevalence of such situations, where environmental stimuli should be processed both based on their relevance as well as their reward value, a large body of literature has sought to investigate the underlying mechanisms of selective attention, reward-driven modulation of perception and the interaction of the two. However, the exact nature of this interaction and its underlying mechanisms have remained unknown (Anderson, 2016b; Anderson et al., 2021; Carrasco, 2011; Failing and Theeuwes, 2018; Maunsell, 2004; Pessoa, 2015).

Converging evidence from behavioral and neurophysiological studies has shown that reward value modulates sensory perception and its neuronal correlates (Baldassi and Simoncini, 2011; Bayer et al., 2017; Hickey et al., 2010b; Hughes et al., 2013; Leo and Noppeney, 2014; Pooresmaeili et al., 2014; San Martín et al., 2016; Serences, 2008). Similarly, selective attention affects behavioral and neural responses to sensory inputs (Desimone and Duncan, 1995). The similarity between reward and attention has raised the question of whether they can be dissociated from each other (Maunsell, 2004). Previous studies have provided divergent answers to this question. On one hand, the majority of past studies have reported a strong interaction between reward and attention (Anderson, 2013; Chelazzi et al., 2013; Failing and Theeuwes, 2018; Le Pelley et al., 2016; Yantis et al., 2012), suggesting a dependence between the two. For instance, neurophysiological studies showed similar effects of reward and attention on neuronal responses of area V1, and that reward effects are gated by attention, inspiring the idea of a 'unified selection signal' comprising both factors (Stanisor et al., 2013). Other studies showed a different form of interaction where the strength of attention to locations or features of stimuli was gated by reward (Chelazzi et al., 2014, 2013; Pessoa, 2015). These studies hence point to a bi-directional interaction between attention and reward, each influencing the strength of the other. This view is also in line with recent suggestions that reward and attention jointly influence 
stimulus representations on an integrated priority map (Chelazzi et al., 2014; Failing and Theeuwes, 2018), although it is unknown how such an integration is implemented. In contrast to this idea, a few studies provided evidence for the independence of reward and attention, as reward effects were unchanged by the amount of attentional load (Baldassi and Simoncini, 2011) or the relevance of reward cue to the task (Garcia-Lazaro et al., 2018), and occurred during separate stages of processing captured by distinct ERP components (Bayer et al., 2017).

Similar to the approach of the latter studies, one possible way to dissociate effects of reward and attention is to manipulate each factor orthogonally to the other. For instance, if successful detection of a target among distractors leads to the delivery of a reward, target features that are predictive of higher reward not only signal a better outcome (i.e., reward) but also engage attentional mechanisms more strongly than low reward cues. However, incidental reward cues that are linked to past rewarding experiences, or predict reward magnitude independent of the performance in a task are more likely to have less dependence on task-related attentional processes. Likewise, orthogonal manipulation of reward and attention can be achieved when reward information is signaled through a stimulus feature (Baldassi and Simoncini, 2011; Garcia-Lazaro et al., 2018) or sensory modality (Pooresmaeili et al., 2014; Vakhrushev et al., 2021) that is distinct from the task-relevant target. Combining these (i.e., using previously rewarded stimuli that are task-irrelevant and are delivered through a different sensory modality) is likely to allow the maximum separation between the reward-related and the attentional prioritization of stimulus processing. In the current study, we try to shed light on the interaction between reward and attention under such conditions.

We draw on previous findings showing that reward cues from a different sensory modality (audition) can affect perception in vision (Pooresmaeili et al., 2014), even when cross-modal reward cues (auditory sounds) are irrelevant to the task at hand (i.e., visual orientation discrimination). Since there is evidence for the separation of attentional resources across sensory modalities (Alais et al., 2006; Duncan et al., 1997), these findings may indicate that cross-modal reward cues affect visual processing independently of visual attention. More recently (Vakhrushev et al., 2021), we showed that behavioral and EEG correlates of reward effects differ between cues that are in the same or different sensory modalities as the target (intra-modal and cross-modal, respectively). Whereas intra-modal reward cues interfered with the processing of target, cross-modal reward boosted the target processing. These results further support the idea that the interaction of attentional and 
reward mechanisms may depend on the sensory modality of the reward with respect to the target of the task. However, in the latter study, intra-modal and cross-modal conditions not only differed in how reward was cued but also in whether they involved processing of a unimodal (only visual) or a bimodal (audiovisual) stimulus. Additionally, in these previous studies selective attention was not systematically manipulated, and therefore the assumed interaction between the sensory modality and attention could not be validated through comparing distinct experimental conditions engaging varying amounts of attention to stimuli.

In the current study, we remedy the shortcomings mentioned above and ask whether the behavioral and neural effects of reward and attention occur independently from each other. Additionally, we ask whether a putative interaction between attention and reward depends on the sensory modality of reward cues. To answer these questions, we modified a behavioral paradigm developed by a previous study Talsma (2005) where it is possible to independently control both attention and reward. In this paradigm, participants performed a change detection task on bimodal, audiovisual stimuli. Spatial attention was manipulated in a block-wise manner, where in each block participants were asked to attend either to the left or to the right visual hemifield and report changes that occurred in the attended hemifield. Reward value was manipulated by associating a specific feature of visual (orientation) or auditory (pitch) cues with different magnitudes of monetary reward (high-value or no-value), resulting in stimuli where either one, both or neither of the modalities were associated with reward. Importantly, these features were orthogonal to the change detection task and were not predictive of the reward delivery as reward associations were learned in a separate conditioning task prior to the test phase. Concurrently with this task, EEG data was recorded allowing us to inspect the effects of reward and attention on visual as well as auditory eventrelated potentials (ERPs). All procedures and hypotheses (referred to as $\mathrm{H} 1-\mathrm{H} 7$ ) were preregistered (https://osf.io/xte4v).

We expected to find that both reward value and allocation of attention $(\mathrm{H} 2)$ enhance behavioral and electrophysiological indices of sensory perception (i.e., a main effect of reward and attention). Additionally, we predicted that there would be no significant interaction between reward and attention (H3). The latter hypothesis was based on two lines of reasoning: firstly, by maximal orthogonalization of reward and attention we predicted that both factors could independently affect sensory perception. Secondly, the task imposed a minimum load on the attentional control as each trial only contained one stimulus and attention was manipulated across blocks. These characteristics were optimal for allowing 
reward information to influence perception both on the attended as well as on the unattended side without a significant cost for the system, hence leading to reward modulations independently of attention. Additionally, we hypothesized that reward effects and their interaction with attention might depend on the sensory configuration of the reward cues; i.e. whether visual, auditory or both visual and auditory cues, are associated with reward (H4/5/6). Specifically, stimuli comprising high reward stimuli in both modalities were predicted to have a higher associative value compared to those that contained a rewarded and an unrewarded cue. Additionally, based on our previous findings, we also expected a difference between intra- and cross-modal reward effects with respect to the sensory areas where reward effects were inspected $(\mathrm{H} 7)$. Therefore, we predicted the more robust modulations of visual ERPs to occur for configurations that contained a high-value visual cue, i.e., when reward cues were intra-modal, than those containing a high-value auditory component. Likewise, when examining auditory ERPs, it was expected that stimuli containing a high-value auditory component would elicit more robust responses. To provide a preview of our results, we found a strong effect of attention (confirming H2) and an interaction between reward and attention (rejecting H3) where attentional modulation of ERPs was stronger for high reward stimuli.

\subsection{Methods}

\section{Participants}

Forty-two volunteers participated in the experiment (mean \pm SD: $27.4 \pm 5.4$ years; 22 females and 20 males). Four participants were removed from the analysis due to their low performance during the behavioral task (accuracy $<60 \%$ ). The sample size was based on a previous study (Vakhrushev et al., 2021) and was set to $\mathrm{N}=33$ but to compensate for possible dropouts and outliers we recorded data from $\mathrm{N}=42$ participants. Subjects were recruited via a local database and had normal or corrected to normal vision. At the end of the experiment, participants received monetary compensation of 8.5 Euros/hour (maximum 35 euros). All procedures were approved by “Universitätsmedizin Göttingen” (UMG) under proposal number 15/7/15.

\section{Procedure}

The experiment was divided into three parts: (1) practice and threshold estimation, (2) learning of a specific association of visual and auditory (e.g., line orientation_1: High Reward, line orientation_2: No Reward, and sound pitch_1: High reward, sound pitch_2: No 
reward) cues with reward followed by the main task, (3) learning of the reversed pairings, where the association of visual and auditory features with reward was reversed, followed by the main task. This way, we ensured that the observed effects originated from the associative learning of reward values and were not due to the pre-existing biases towards a certain stimulus feature. The main task required participants to detect a transient change in the intensity of visual or auditory cues that occurred in $20 \%$ of trials (see below).

\section{Training and threshold estimation}

At the beginning of each session, participants were first familiarized with the change detection task through performing 160 practice trials. The practice session contained audiovisual stimuli with frequent changes ( $80 \%$ of trials); i.e. dimming of the luminance of visual stimuli and reduction in the sound pressure of auditory tones happening midway through their presentation, that participants had to detect. Participants were instructed to detect the change on both sides of the screen and the difficulty of the task was gradually increased by decreasing the intensity of the change. The experiment proceeded only after participants felt confident performing the task.

During the threshold estimation phase, participants were instructed to detect the change in a unimodal stimulus (i.e., either visual or auditory) on both sides of the screen. There were five levels of change intensity, which were randomized across trails. The estimation of threshold was done in blocks with 90 trials separately for visual and auditory cues. There were three blocks of threshold estimation: estimation of change detection threshold for the low pitch sound, high pitch sound, and visual cues. The order of these blocks was randomized across participants. Responses were fed to a QUEST procedure (Watson and Pelli, 1983), a built-in standard package of Psychophysics toolbox-3 (Brainard, 1997), where the smallest amount of change in stimulus intensity to achieve a certain level of performance was estimated. Based on the methods of a previous study, the threshold was set to $90 \%$ accuracy.

\section{Stimuli and task}

\section{Main Task}

At the beginning of each block (180 trl) of the task, participants were instructed to fixate their eyes on the screen center and pay attention to only one side (either left or right, counterbalanced across blocks). The task was to detect target trials, i.e., trials containing a change, by pressing a "Space" button on a keyboard. Target trials were randomly interleaved with the rest of the trials and constituted $20 \%$ of all trials. Target trials were similar to 
standard trials, except that halfway through the presentation of stimuli a transient drop of stimulus intensity occurred, which caused an impression of stutter (an auditory cue) or flicker (visual cue).

Every trial started with a fixation period $(750 \mathrm{~ms}-1050 \mathrm{~ms})$ followed by the presentation of a bimodal (audio-visual) stimulus (for $108.3 \mathrm{~ms}$, on a gray background), either on the left or right side of the screen. All stimuli were lateralized $15^{\circ}$ relative to the vertical meridian and $6^{\circ}$ below the horizontal meridian. Auditory stimuli were either a $1050 \mathrm{~Hz}$ or a $350 \mathrm{~Hz}$ tone delivered through in-ear headphones and processed with HRTF filters to render them co-localized with the visual stimuli (Algazi et al., 2001), with a linear rise and fall of $10 \mathrm{msec}$ and an amplitude of $75 \mathrm{~dB}$. Visual stimuli were tilted square-wave gratings $\left(6^{\circ}\right.$ visual degrees or about $\left.5.8 \times 5.8 \mathrm{~cm}\right)$, modulated between white and gray colors, and oriented $\pm 45^{\circ}$.

In total, trials during the test phase were divided into eight conditions with four attended and four unattended conditions and four different reward value configurations. The latter consisted of: (1) visual high-value and auditory no-value: VHSN (2) visual no-value and auditory high-value: VNSH (3) visual high-value and auditory high-value: VHSH (4) visual no-value and auditory no-value VNSN (see Figure 1). Additionally, a no-stimulus condition was added where no stimulus was presented and only the fixation point was displayed on the screen for the entire duration of the trial. This condition was added to eliminate the overlapping electrophysiological activity across consecutive trials (Woldorff, 1993), as implemented by (Talsma and Woldorff, 2005). Participants completed 3600 trials during the main task where each of the reward conditions was repeated 400 times (200 trials per screen side). Of these, target trials (only on the instructed location) constituted $10 \%$ of all trials (i.e., 40 trials).

a) Conditioning Phase

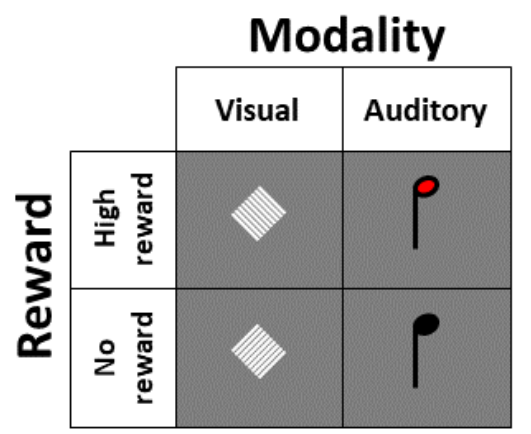

b) Main Task

\section{Modality with high reward}

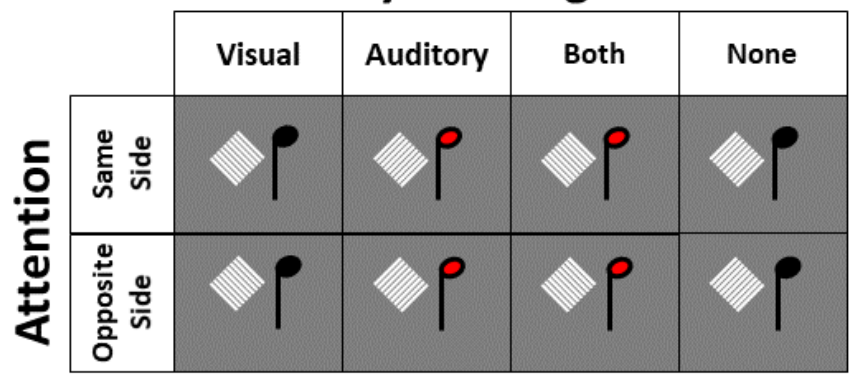

Figure 1. Stimuli and design of the experiment. The experiment has two phases: the conditioning phase, where the reward value of unimodal auditory or visual cues was learned, and the main task when the previously rewarded auditory and visual stimuli were presented together. a) Conditioning phase contained two independent factors: cue modality (visual or auditory) and cue reward value (high reward or no reward). b) In 
the main task two independent factors were manipulated: modality with high reward value (visual, auditory, both, none) and attention (same side or opposite side relative to the audiovisual stimulus).

\section{Conditioning phase}

During the conditioning phase, participants associated different tones and line orientations with positive or no reward outcomes (high-value or no-value, respectively). Similar to the main task, participants had to detect the target trials containing a change. However, there were a few key differences. First, all trials in the conditioning phase were unimodal (only visual or only auditory) and grouped in blocks of 27 trials by the modality. Blocks with different modalities were randomized across conditioning phase. Second, all trials in conditioning phase were followed by a monetary reward outcome (displayed for 500 ms) proportional to the value of the cue presented in that trial. Participants were instructed to remember the reward outcome of each condition and later report it (at the end of each block in the conditioning phase, through a $2 \mathrm{AFC}$ procedure in which the high reward stimulus of two consecutively presented stimuli had to be reported). These rewards were drawn from two Poisson distributions with the mean of 40 cents and SD of 5.5 cents for high-reward trials (maximum reward was fixed at 50) and 0 cents for no-reward trials. Third, participants were instructed to report target trials (trials with a transient change) on both sides of the screen.

In total, the conditioning phase had four conditions: (1) high-value visual, (2) Highvalue auditory, (3) no-value visual, and (4) no-value auditory (Figure 1). These conditions were divided into factors modality (visual or auditory) and reward (high- or no-value). Similar to the main task, a no-stimulus condition was added. Participants completed 1200 trials during the conditioning phase, where each condition was repeated 240 times (120 trials per screen side). $20 \%$ of all trials were targets (trials with a transient change). Finally, to control for the perceptual biases towards visual and auditory cues, this phase was repeated twice and in the second repetition the reward pairings were reversed. This was indicated to participants with an instruction display before the second repetition commenced.

\section{Apparatus}

The experiment was conducted in a darkened, sound-attenuated, and electromagnetically shielded chamber. Participants were seated on a chair with their heads fixed on a chinrest positioned $80 \mathrm{~cm}$ from a $22.5^{\prime}$ monitor (refresh rate $=120 \mathrm{~Hz}$ ). Stimulus presentation was controlled by a PC under the Windows operating system equipped with MATLAB (version R2015b) and Psychophysics toolbox-3 (Brainard, 1997). 
This study assessed the accuracy and reaction times (RT) of the detection of target stimuli (containing a transient change). Eye movements were recorded with an Eyelink 1000 eye tracker system (SR Research, Ontario, Canada) in a desktop mount configuration, recording the right eye at a sampling rate of $1000 \mathrm{~Hz}$. The electrophysiological (EEG) data was continuously recorded from 64 electrodes with an actiChamp system and referenced to the A2 electrode. The recording was done with BrainVision software (BrainVision Recorder 1.23.0001 Brain Products GmbH, Gilching, Germany; actiCap, Brain Products GmbH, Gilching, Germany). The signal was digitized at $1000 \mathrm{~Hz}$ and amplified with a gain of 10,000. All electrode/skin impedances were kept below $10 \mathrm{k} \Omega$.

\section{Transformations}

\section{Behavioral data}

Reaction times (RTs) of correctly detected target stimuli and their accuracy were computed separately for each condition at each phase of the experiment.

\section{ERP analysis}

EEG data was imported and processed offline using EEGLAB (Delorme and Makeig, 2004), an open-source toolbox running under the MATLAB environment. Raw data of each participant was band-pass filtered with $0.1 \mathrm{~Hz}$ as the high-pass cutoff and $40 \mathrm{~Hz}$ as the lowpass cutoff frequencies. An automatic bad channel detection algorithm was applied using EEGLAB's pop_rejchan method (threshold $=5$, method $=$ kurtosis). All trials detected online as a keypress or fixation breaks (eye position to the fixation point $>9^{\circ}$ ) were removed from the analysis. After this, epochs were extracted using a stimulus-locked window of 3000 ms (1000 to $2000 \mathrm{~ms}$ ). The remaining data were subjected to an Independent Component Analysis (ICA) algorithm (Delorme and Makeig, 2004). Eye-blinks and eye-movements artifacts were automatically identified and corrected using an ICA-based automatic method, implemented in the ADJUST plugin of EEGLAB (Mognon et al., 1994). Bad channels were interpolated by using the default spherical interpolation method. Data were re-referenced offline to the average reference. Finally, stimulus-locked epochs were extracted using a window of $1100 \mathrm{~ms}$ (-100 to $1000 \mathrm{~ms}$ ), and baseline corrected using the pre-stimulus time interval (-100 to $0 \mathrm{~ms})$. The stimulus-locked epochs were extracted using a window of 2200 $\mathrm{ms}(-1000$ to $1200 \mathrm{~ms})$, and baseline corrected using the pre-stimulus time interval (-100 to $0 \mathrm{~ms})$

Our ERP analysis was focused only on standard trials, i.e., trials without a change, as done previously (Talsma and Woldorff, 2005). Visual ERPs were measured in occipital 
electrodes (PO7/8 O1/2) for P1 (70-170 ms) and N1 (180-250 ms) components. P300 (350$600 \mathrm{~ms}$ ) responses were measured over $\mathrm{Pz}$, and $\mathrm{CNV}$ (contingent negative variation) component (650-1000 ms) over central electrodes (Fz, Cz, Pz) (P300 and CNV as done in Pfabigan et al. (2014). Auditory N1 component was measured at Fz electrode and within the same time window as visual ERPs. Analysis of visual P1 and N1 and auditory N1 components was based on the average within $60 \mathrm{~ms}$ window around the respective peak of each component measured across participants for each condition. Before the ERP analysis, we removed overlapping ERP activity from adjacent trials by subtracting a waveform of a no-cue condition from a waveform of each condition with an audiovisual cue, as Talsma et al. (2005) implemented. In this chapter we will only focus on P1 component measured in occipital electrodes and auditory N1 measured in Fz.

a) Conditioning Phase

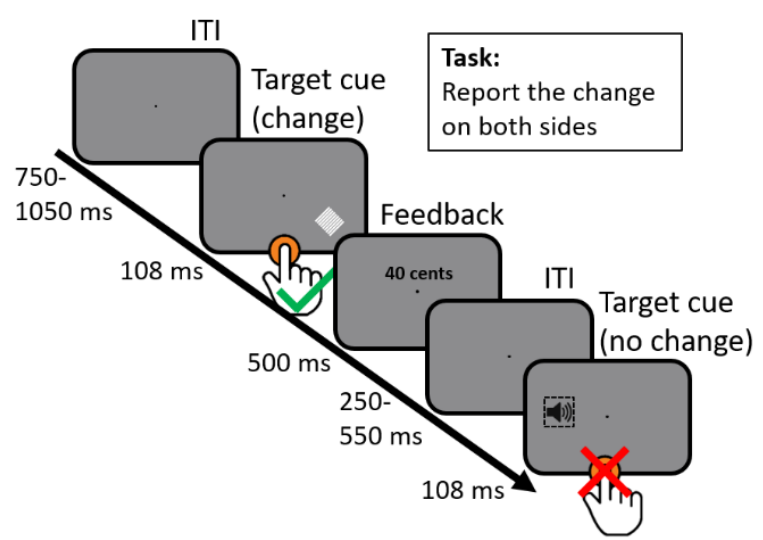

b) Main Task

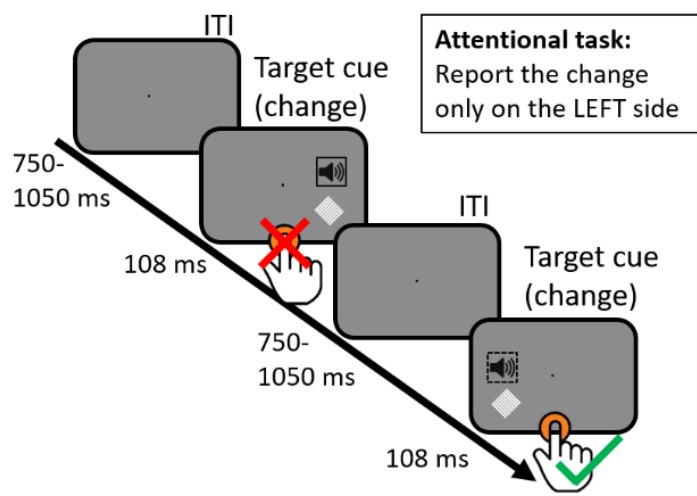

Figure 2. The sequence of trial events. Participants were instructed to detect occasional targets by pressing a "space bar button". Targets were trials with a change in intensity of auditory and/or visual stimuli midway through their presentation. The change made an impression of a stutter (auditory condition) or a flicker (visual condition). Both visual $\left( \pm 45^{\circ}\right.$ tilt $)$ and auditory stimuli $(350 \mathrm{~Hz}$ and $1050 \mathrm{~Hz})$ were presented laterally $\left(15^{\circ}\right)$ and below the fixation point $\left(6^{\circ}\right)$. a) The experiment started with a conditioning phase in which reward associations were learned. b) In the main task, visual and auditory cues always appeared together: unilaterally and synchronously. At the beginning of every block with the main task, participants were instructed to pay attention only to the one side of the screen and ignore target trials on the other side (in the demonstration above participants were instructed to only report changes on the left side).

\section{Analysis}

\section{Behavioral data}

Statistical analyses were done separately for the conditioning phase and the main task. In the conditioning phase, a two-way repeated-measures analysis of variance analysis (ANOVA) of RTs was done with factors modality (visual or auditory) and value (high-value cue or no-value cue).

In the main task, all cues were bimodal, where individual sounds and visual cues were either associated with high-value or no-value (VHSH - visual cue has high-value and sound 
has high-value; VHSN - visual cue has high-value and sound has no-value; VNSH - visual cue has no-value and sound has high-value; VNSN - visual cue has no-value and sound has no-value). Here a two-way ANOVA was used with the factor auditory reward value (high or no) and visual reward value (high or no). Significant main or interaction effects were subsequently inspected using planned pairwise comparisons.

EEG data during the main task

Firstly, to test whether we replicate the reported effects of a previous study that had inspired our paradigm (Talsma and Woldorff, 2005), ERP responses to the attended and unattended stimuli across all conditions were compared (Figure 4). Subsequently, we conducted multiple repeated-measured ANOVA (rmANOVA) to test different aspects of the effects of attention and reward on visual P1 and auditory N1 components.

A rmANOVA comprising factors attention (attended or unattended) and value (highor no-value) was done on the data of conditions in which both visual and auditory cues of bimodal stimuli had the same value; i.e., either high- or no-value (VHSH and VNSN). Here, a main effect of reward would confirm hypothesis 1, a main effect of attention would confirm hypothesis 2, the absence of interaction effects would confirm hypothesis 3 of our preregistered plan (Figure 5).

Having established an effect of attention in previous two analyses, we next subtracted the ERPs of attended and unattended conditions and tested the effect of reward value separately in each modality (Figure 6). To this end, a two-way rmANOVA with factors value in visual modality (high-value or no-value), value in auditory modality (high-value or no-value) was done separately on visual P1 and auditory N1 components (hypothesis 4-6).

Finally, to test whether the effect of value in visual or auditory modalities differs between different regions of interest (ROI), we conducted a three-way rmANOVA with factors ROI (two levels: visual (PO7, O1, O2, PO8) or frontal region (Fz)), value in visual modality (high-value or no-value), value in auditory modality (high-value or no-value). Since P1 and N1 components have an opposite amplitude polarity, their absolute values were included in this analysis. Here, our focus was on testing whether an interaction existed between value in each modality and the ROI, testing a prediction (hypothesis 7) that reward value from each modality will have the strongest effect in its respective specialized modality, i.e., intra-modally. 


\subsection{Results}

\section{Behavioral results}

We first verified that during the conditioning phase, visual and auditory reward cues modulated response times (RTs) indicating that participants had learned the value associations. An ANOVA with factors value (high- or no-value cue) and modality (visual or auditory) revealed a main effect of modality $\mathrm{F}(1,37)=13.9, p<0.001, \eta_{\mathrm{p}}{ }^{2}=0.27$ and a main effect of value $\mathrm{F}(1,37)=12, p<0.001, \eta_{\mathrm{p}}{ }^{2}=0.25$, as well as an interaction effect $\mathrm{F}(1,37)=$ $5, p=0.031, \eta_{\mathrm{p}}{ }^{2}=0.12$. Participants were faster at detecting the change in the visual cues (mean \pm s.e.m.: $486.06 \pm 6.49$ ) compared to the change in the auditory cue (mean \pm s.e.m.: $506.41 \pm 6.08)$. Similarly, participants were faster at detecting the change in high-value cues (mean \pm s.e.m.: $490.97 \pm 5.94$ ) compared to the change in no-value cues (mean \pm s.e.m.: $501.49 \pm 5.79$ ). A follow-up pairwise comparison revealed that these reward effects were strongest for auditory cues $\left(\mathrm{t}(37)=-3.25, p<0.005\right.$, Cohen's $\mathrm{D}\left(\mathrm{d}_{\mathrm{z}}\right)=0.53$ ), where response to a high-value auditory cue (mean \pm s.e.m.: $497.25 \pm 6.40$ ) was much faster than to a novalue auditory cue (mean \pm s.e.m.: $515.56 \pm 6.99$ ). The difference between high- and novalue visual cues did not reach significance $(\mathrm{t}(38)=-0.83, p=0.41)$. Nevertheless, the direct assessment of reward associations during and after conditioning phase indicated that participants successfully learned reward values of both visual and auditory cues. All participants, when offered to choose a cue with more money, selected high-value cues.

Behavioral results from the main task are shown in Figure 3. In behavioral analysis, our interest lay in how previously rewarding visual and auditory cues affected response times. A two-way rm ANOVA with the factor visual reward value (2 levels) and auditory reward value (2 levels) revealed a main effects of auditory reward value $\mathrm{F}(1,37)=5.36, p$ $<0.05, \eta_{\mathrm{p}}{ }^{2}=0.127$. Response time to high-value auditory cues was faster (mean \pm s.e.m.: $484.99 \pm 7.23$ ) compared to no-value auditory cues (mean \pm s.e.m.: $492.46 \pm 8.40$ ). We found no value effect in visual modality $(p=0.15)$. Follow-up pairwise comparisons revealed that the reward effect in auditory cues originated from two differences. One difference was between the condition with high-value cue in both modalities (VHSH) and the condition with high-value only in vision, i.e., VHSN $\left(\mathrm{t}(37)=2.28, p<0.05, \mathrm{~d}_{\mathrm{z}}=0.37\right)$. The second difference was between a condition with high-value only in auditory modality and a condition with high-value only in vision; i.e. VNSH and VHSN ( $(37)=2.99, p<0.005, \mathrm{~d}_{z}$ $=0.49$ ). Both in VHSH (mean \pm s.e.m.: $485.47 \pm 6.93$ ) and VNSH (mean \pm s.e.m.: $484.50 \pm 7.73$ ) conditions, participants responded faster than VHSN (mean \pm s.e.m.: 494.14 $\pm 8.65)$. 
Therefore, in the main task, we found faster responses to stimuli that contained a highvalue auditory cue compared to conditions that contained a low-value auditory cue. This difference was strongest when comparing VNSH and VHSH against VHSN condition, as the latter had the most prolonged response times. Together, the analysis of behavioral data in conditioning and main task indicate a strong influence of reward value cued through the auditory modality on the target detection.

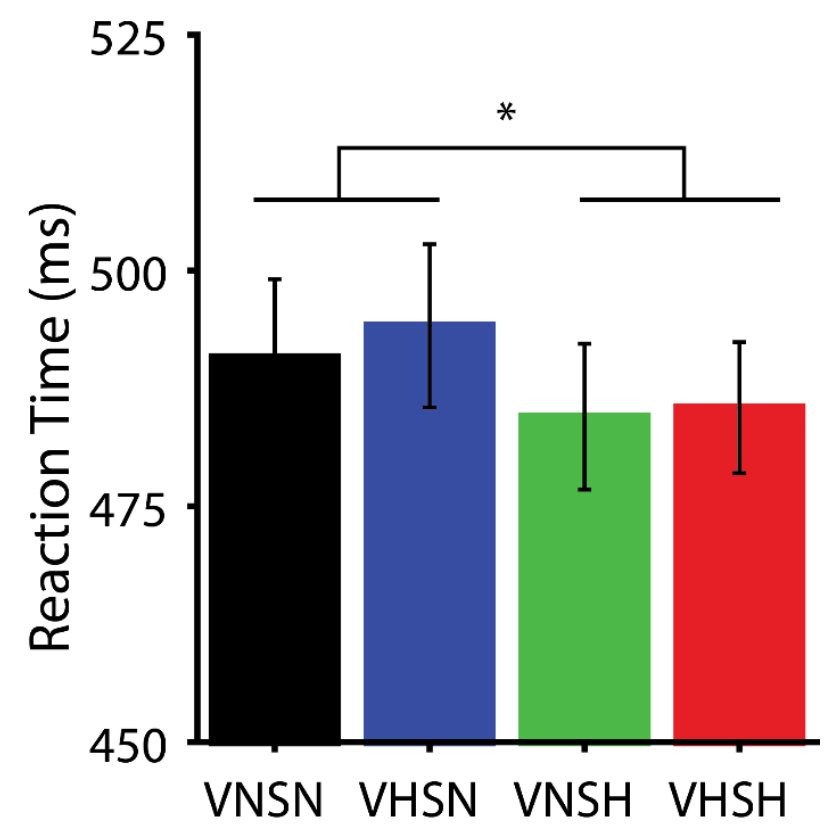

Figure 3. Reaction times during the main task. Reaction times are only shown for correctly detected trials. Four stimulus configurations consisted of conditions with high reward value in both modalities (VHSH), only in visual modality (VHSN), only in auditory modality (VNSH), or in neither of the modalities (VNSN). 


\begin{tabular}{|l|c|c|c|c|}
\hline & VNSN & VHSN & VNSH & VNSN \\
\hline $\begin{array}{l}\text { Response Time } \\
\text { (RT) }\end{array}$ & $490.79 \pm 8.28$ & $494.14 \pm 8.65$ & $484.50 \pm 7.73$ & $485.47 \pm 6.93$ \\
\hline $\begin{array}{l}\text { Visual P1 } \\
\text { amplitude } \\
\text { (attended } \\
\text { condition) }\end{array}$ & $3.07 \pm 0.25$ & $3.15 \pm 0.25$ & $3.13 \pm 0.24$ & $3.15 \pm 0.24$ \\
\hline $\begin{array}{l}\text { Visual P1 } \\
\text { amplitude } \\
\text { (unattended } \\
\text { condition) }\end{array}$ & $2.61 \pm 0.23$ & $2.70 \pm 0.22$ & $2.61 \pm 0.23$ & $2.50 \pm 0.23$ \\
\hline $\begin{array}{l}\text { Auditory N1 } \\
\text { amplitude } \\
\text { (attended } \\
\text { condition) }\end{array}$ & $-2.07 \pm 0.18$ & $-2.13 \pm 0.18$ & $-2.14 \pm 0.17$ & $-2.20 \pm 0.19$ \\
\hline $\begin{array}{l}\text { Auditory N1 } \\
\text { amplitude } \\
\text { (unattended } \\
\text { condition) }\end{array}$ & $-1.93 \pm 0.17$ & $-1.98 \pm 0.15$ & $-1.89 \pm 0.16$ & $-1.82 \pm 0.17$ \\
\hline
\end{tabular}

Table 1. Overview of behavioral and electrophysiological indices measured in each stimulus condition.

\section{Electrophysiological markers of attention and reward value}

Attentional modulation of ERP responses

We first tested whether attentional modulation effects reported by Talsma et al. (2005) are replicated in our paradigm, as shown in Figure 4. A one-way ANOVA on visual P1 component $(\mathrm{PO} 7, \mathrm{O} 1, \mathrm{O} 2, \mathrm{PO})$ revealed a main effect of attention $(\mathrm{F}(1,37)=64.34, p<$ $0.001, \eta_{\mathrm{p}}{ }^{2}=0.635$ ). Attended conditions increased the amplitude P1 component (mean $\pm \mathrm{SD}$ : $3.12 \pm 0.24$ ) compared to unattended conditions (mean $\pm \mathrm{SD}: 2.60 \pm 0.22$ ). A similar effect was found in auditory $\mathrm{N} 1$ component measure at $\mathrm{Fz}\left(\mathrm{F}(1,37)=15.53, p<0.001, \eta_{\mathrm{p}}{ }^{2}=0.296\right)$, where attended cues increased the ERP amplitude of N1 component (mean \pm SD: $-2.13 \pm$ 0.17 ) compared to unattended cues (mean $\pm \mathrm{SD}$ : $-1.90 \pm 0.15$ ). These results replicate previous findings (Talsma and Woldorff, 2005) and indicate that our experimental design modulated the allocation of spatial attention successfully. 
a)

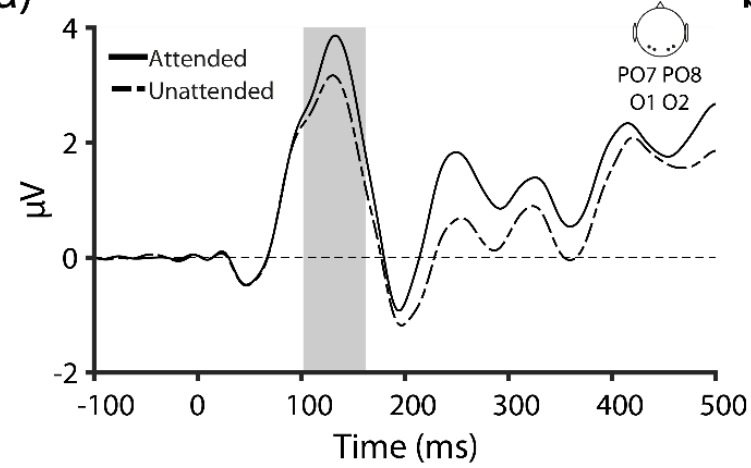

b)

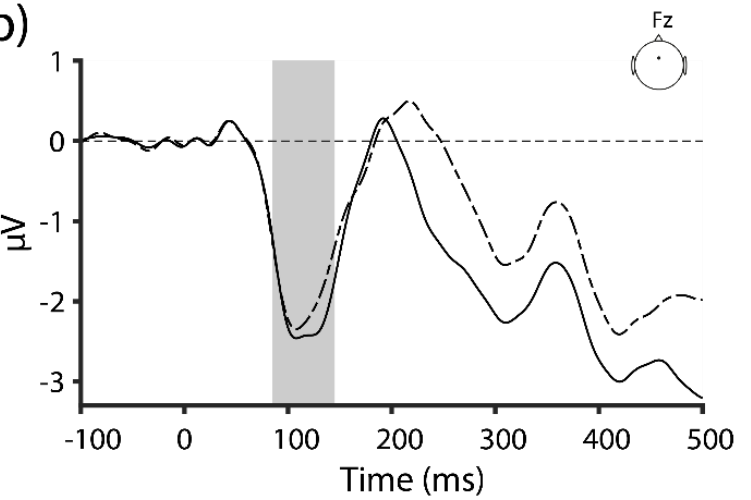

Figure 4. Attentional modulation of ERPs. a) ERP waves produced by attended and unattended audiovisual cues that were recorded over occipital electrodes (PO7, O1, O2, PO8) or b) frontal electrodes (Fz).

\section{Interaction between attention and reward and modulation of ERP responses}

Visual P1 component

Next, we examined the attentional and reward effects in conditions in which both modalities had either high- or no-value (VHSN and VNSN). Figure 5a,c shows ERP waves in occipital region (PO7,O1,O2,PO8) of high- and no-value conditions when they were attended or unattended. Here an analysis of P1 component revealed a main effect of attention $\left(\mathrm{F}(1,37)=47.1, p<0.001, \eta_{\mathrm{p}}{ }^{2}=0.56\right)$, no main effect of reward value $(\mathrm{F}(1,37)=0.03, p=$ $0.86)$ and an interaction effect between attention and reward value $(\mathrm{F}(1,37)=4.99, p=$ $0.032, \eta_{\mathrm{p}}^{2}=0.12$ ). As mentioned above, attended cues evoked higher amplitude of P1 component (mean \pm s.e.m.: $3.11 \pm 0.24$ ), compared to unattended cues (mean \pm s.e.m.: 2.56 \pm 0.23 ). The interaction effect was due to the interdependence of attention and reward effects. Firstly, the difference between attended and unattended conditions was larger when cues had high-value (mean \pm s.e.m.: $0.64 \pm 0.09$ ) compared to no-value (mean \pm s.e.m.: 0.46 \pm 0.09 ). Secondly, the difference between high- and no-value conditions was in different directions depending on whether they were attended (mean \pm s.e.m.: $0.08 \pm 0.10$ ) or unattended (mean \pm s.e.m.: $-0.11 \pm 0.09$ ). However, follow-up pairwise comparisons revealed that only the difference between attention conditions was significant: the attended high-value stimulus (mean \pm s.e.m.: $3.15 \pm 0.24$ ) evoked a significantly $(\mathrm{t}(37)=6.9, p<$ $0.001, \mathrm{~d}_{\mathrm{z}}=1.12$ ) higher P1 amplitude compared to the unattended high-value stimulus (mean \pm s.e.m.: $2.50 \pm 0.23)$. Similarly the attended no-value stimulus evoked significantly (t(37) $=5.2, p<0.001, \mathrm{~d}_{\mathrm{z}}=0.85$ ) higher P1 amplitudes (mean \pm s.e.m.: $3.07 \pm 0.25$ ) compared to the unattended no-value stimulus (mean \pm s.e.m.: $2.61 \pm 0.23$ ). 


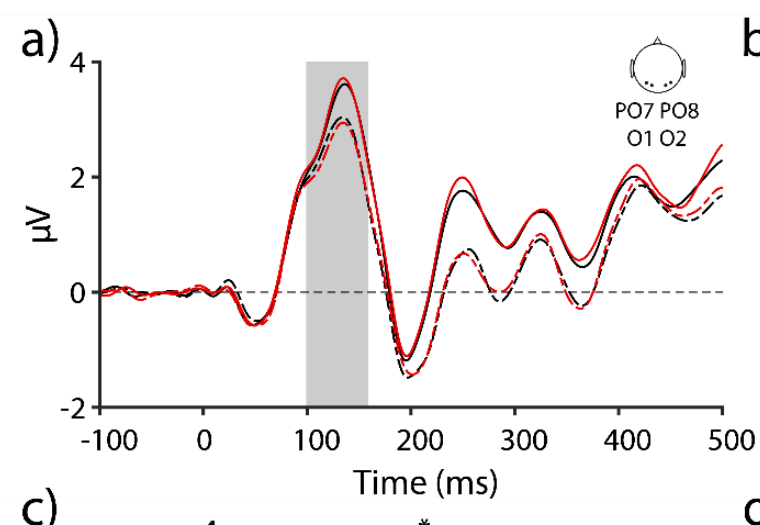

c)

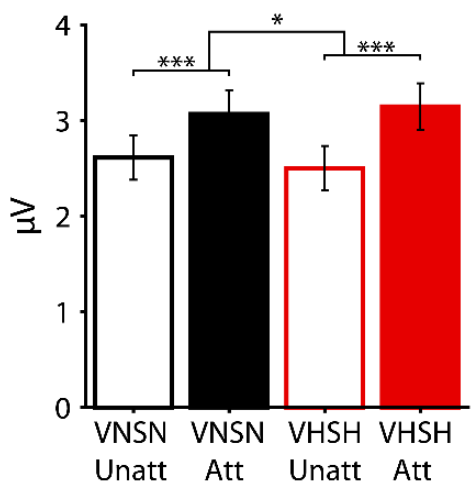

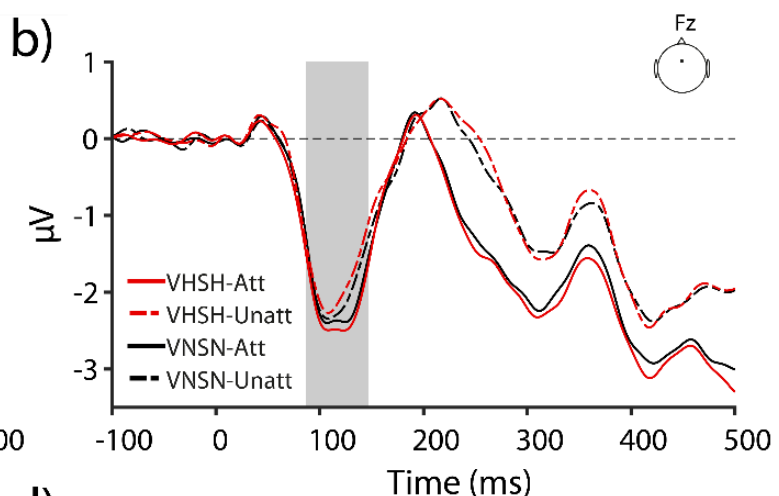

d)

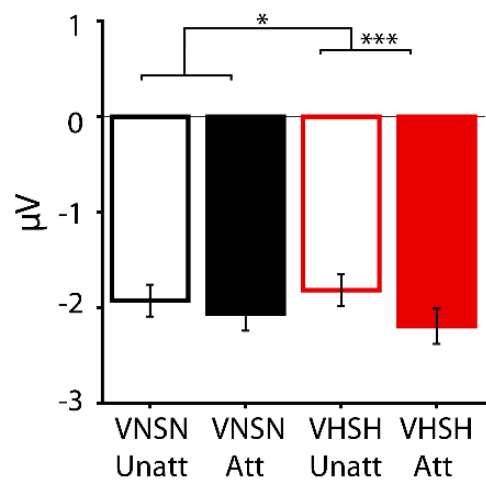

Figure 5. Modulation of ERPs by attention and reward factors. ERP waves measured over occipital (a) or frontal (b) regions for conditions that either had high reward value in both modalities (VHSH) or no reward values in both modalities (VNSN) and were either presented on the attended (Att) or unattended (Unatt) side. c) Same as a) depicting the average visual P1 amplitudes. d) Same as b) depicting auditory N1 amplitudes.

\section{Auditory N1 component}

Similar to the P1 component, an analysis of N1 component (Figure 5b, d) measured in frontal region $(\mathrm{Fz})$ revealed a main effect of attention $\left(\mathrm{F}(1,37)=9.2, p<0.005, \eta_{\mathrm{p}}{ }^{2}=0.2\right)$, no effect of reward value $(\mathrm{F}(1,37)=0.01, p=0.91)$, and an interaction effect between attention and reward value $\left(\mathrm{F}(1,37)=6.10, p<0.05, \eta_{\mathrm{p}}{ }^{2}=0.142\right)$. Attended cues evoked a more negative N1 peak (mean \pm s.e.m.: $-2.13 \pm 0.18$ ) compared to unattended cues (mean \pm s.e.m.: $-1.87 \pm 0.16)$. An interaction between attention and reward indicates that high-value cues resulted in a larger difference in N1 amplitude between attended and unattended conditions (mean \pm s.e.m.: $-0.38 \pm 0.09$ ) compared to no-value cues (mean \pm s.e.m.: $-0.14 \pm$ 0.10). On another side, high-value cues enhanced the amplitude of the N1 peak (mean \pm s.e.m.: $-0.13 \pm 0.08$ ) when they were attended and suppressed it when they were unattended (mean \pm s.e.m.: $0.11 \pm 0.11$ ). Follow-up pairwise comparisons revealed that attended highvalue cues $\left(\mathrm{t}(37)=4.1, \mathrm{p}<0.001, \mathrm{~d}_{\mathrm{z}}=0.66\right)$ evoked higher $\mathrm{N} 1$ amplitudes (mean \pm s.e.m.: $-2.20 \pm 0.19$ ) compared to unattended high-value cues (mean \pm s.e.m.: $-1.82 \pm 0.17$ ) Notably, the difference between attended and unattended no-value cues did not reach significance $(p=0.188)$. 
Together, the results obtained in P1 and N1 components showed a main effect of attention and interaction between attention and reward. A stronger attentional modulation for high-value stimuli demonstrates that stimulus value influences the allocation of attention. Different directions of reward modulations dependent on attention indicate that attention influences the processing of reward information, but the difference between individual reward conditions was non-significant.

\section{Dependence of the reward-driven modulation of attention on the sensory modality of reward cues}

We have so far shown a strong effect of attention on ERP components that is influenced by stimulus reward. We next asked whether this effect depends on the specific configuration of stimuli; i.e., the modality through which reward value is cued (Figure 6). To this end, the ERPs of attended and unattended conditions were subtracted from each other and entered into an ANOVA with factors visual reward value and auditory reward value.

Analysis of visual P1 component revealed no main effect of visual reward value $(\mathrm{F}(1,37)=0.88, p=0.35)$ and a trend for a main effect of auditory reward value $(\mathrm{F}(1,37)=$ $3.49, p=0.07)$. No interaction effect was found between these factors $(\mathrm{F}(1,37)=0.67, p=$ $0.42)$.

Analysis of auditory N1 component showed a main effect of auditory reward value $\left(\mathrm{F}(1,37)=5.64, p=0.023, \eta_{\mathrm{p}}{ }^{2}=0.13\right)$ as the presence of high-value auditory cues increased the N1 amplitude (mean \pm s.e.m.: $-0.32 \pm 0.05 \mu \mathrm{V}$ ) compared to conditions that contained a no-value auditory cue (mean \pm s.e.m.: $-0.14 \pm 0.08 \mu \mathrm{V}$ ). The main effect of visual reward value $(\mathrm{F}(1,37)=0.94, p=0.34)$ and the interaction of visual and auditory reward value were not significant $(\mathrm{F}(1,37)=0.57, p=0.45)$.

Inspection of individual stimulus configuration (see Table 1 and Figure 6) reveals that both P1 and N1 had the highest amplitude when visual and auditory cues were associated with high-value (VHSH), followed by high-value only in auditory modality (VNSH, see also Table 1) whereas other configurations (VHSN and VNSH) elicited lower amplitudes. Pairwise comparisons on auditory N1 where a main effect of auditory reward value was found showed that VHSH elicited significantly higher N1 amplitude (mean \pm s.e.m.: $-0.38 \pm$ $0.09 \mu \mathrm{V})$ compared to both VHSN $\left(\Delta=0.24, \mathrm{t}(37)=2.47, p=0.018, \mathrm{~d}_{\mathrm{z}}=0.4\right)$ and VNSH $\left(\Delta=0.23, \mathrm{t}(37)=2.23, \mathrm{p}=0.032, \mathrm{~d}_{\mathrm{z}}=0.36\right)$. Other pairwise comparisons did not reach significance level (all $p s>0.1$ ).

Finally, to test whether the influence of auditory and visual reward value depended on the region of interest (ROI), we conducted an ANOVA on the absolute values of visual P1 
and auditory $\mathrm{N} 1$ components. This analysis revealed a main effect of $R O I(\mathrm{~F}(1,37)=21.9, p$ $\left.<0.001, \eta_{\mathrm{p}}{ }^{2}=0.37\right)$ as expected, and a main effect of auditory reward value $(\mathrm{F}(1,37)=6.12$, $\left.p<0.05, \eta_{\mathrm{p}}{ }^{2}=0.14\right)$. Importantly, no interaction was found between the auditory or visual reward value and ROI, indicating that auditory reward value enhanced sensory processing both intra-modally (i.e., in Fz) as well as cross-modally (in occipital ROI).

a)

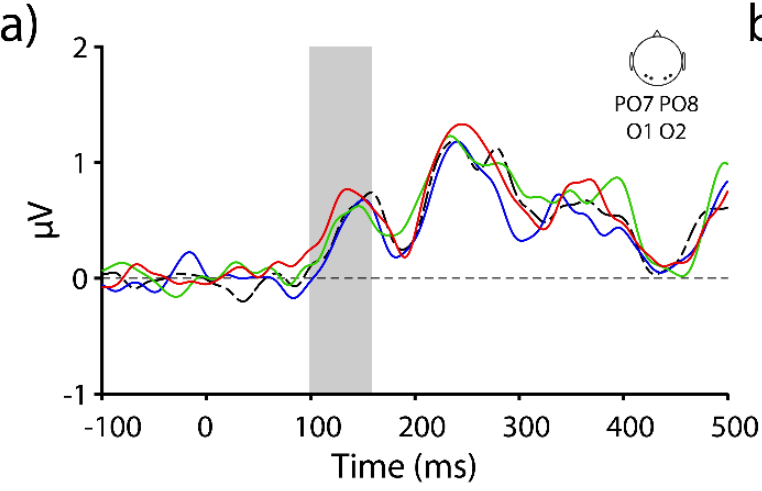

c)

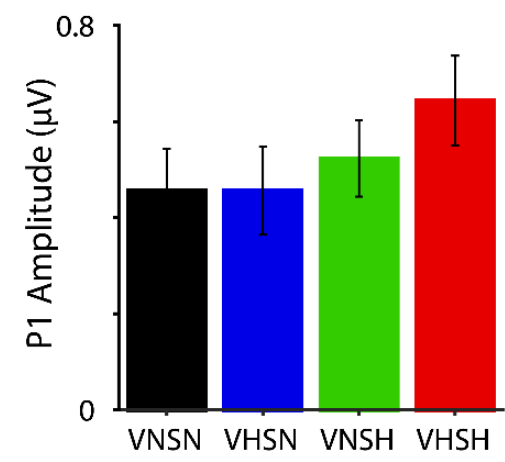

b)

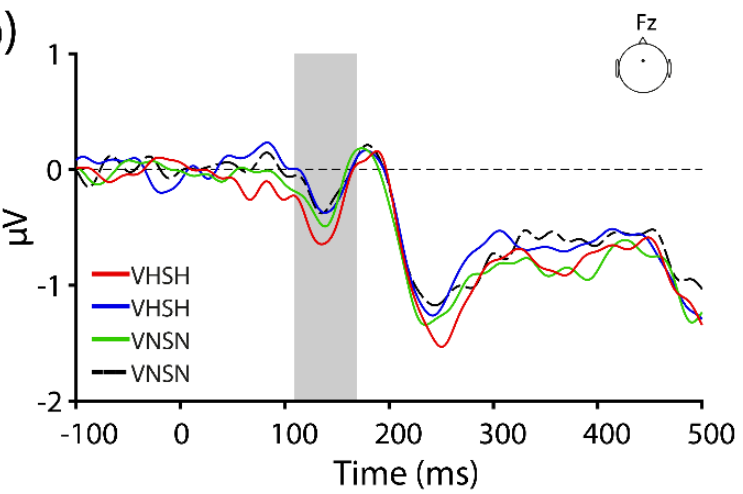

d)

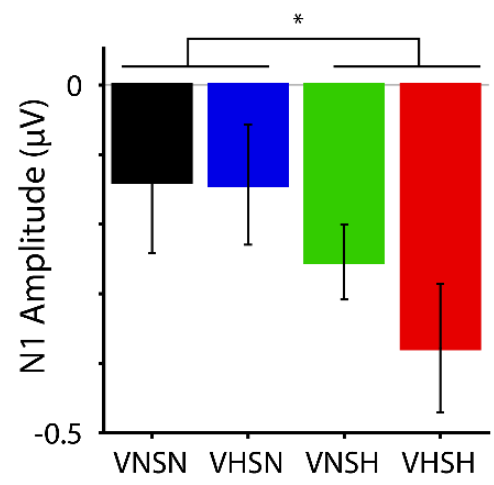

Figure 6. Modulation of attention by reward value of auditory and visual modalities. a) The difference wave between attended and unattended reward conditions (high reward in vision: VHSN, in auditory: VNSH, in both: VHSH, or in neither: VNSN modality) measured in occipital electrodes (PO7, O1, O2, PO8). b) Same as (a) for Fz electrode.

\subsection{Discussion}

The current study examined the interaction between spatial attention and reward value when visual, auditory, or both modalities were associated with high reward. During the associative learning phase, both visual and auditory cues sped up the target detection, with a stronger effect in auditory modality. During the main task, we found a strong effect of attention on early event-related responses of occipital and frontal areas (visual P1 and auditory N1 components, respectively), thus replicating a previous study that inspired our design (Talsma and Woldorff, 2005). We did not find a main effect of reward value, but importantly a significant interaction was found as attentional modulation of P1 and N1 responses were stronger when both modalities had high value compared to no value. These findings suggest that reward value modulates the strength of attentional filtering. However, when visual and auditory reward effects were compared separately, a more robust reward 
modulation of attention was found for the latter, thus reflecting the dominant role of auditory reward signals in the guidance of attention. Finally, our results also revealed that attentional modulation of the ERPs was more robust when both compared to only one of the unimodal components of an audiovisual stimulus were associated with reward. Hence, in the context of our paradigm stimuli with high value, especially in auditory domain, guide the attentional resources towards the attended location and withdraw them from the unattended location.

A host of previous studies have demonstrated that reward biases selective attention towards stimuli previously associated with a positive outcome, an effect observed for shapes (Della Libera and Chelazzi, 2009, 2006), faces (Raymond and O'Brien, 2009), orientations (Kiss et al., 2009), and semantic information in Stroop tasks (Krebs et al., 2010). However, it is not always possible to determine the degree to which the observed behavioral or neural modulations are due to attention, reward, or both (Maunsell, 2004). Often reward-associated stimuli appear as the target of the task or contain target-related information, hence inadvertently engaging attention. More recent studies showed that even when reward stimuli are task-irrelevant (Garcia-Lazaro et al., 2018) or are delivered through a different sensory modality (Pooresmaeili et al. 2014) target processing is influenced by the reward information, suggesting independence of reward and attentional mechanisms. Specifically, a lack of influence of attentional load on reward effects (Baldassi and Simoncini, 2011) and temporally distinct ERP modulations for reward and attention (Bayer et al., 2017) supported the idea that selective attention and reward can influence perception independently. Here, we tried to resolve this ambiguity but while we had predicted an independence of attention and reward, we found evidence to the contrary. In contrast to previous studies that showed a clear separation between effects of reward and attention, our results show that reward effects on early perception occur exclusively through the modulation of attention. We note several major differences between the latter studies and ours. Firstly, Baldassi et al. (2011) employed a weak manipulation of attention, i.e. by performing a secondary task at fixation, compared to ours where attention was strictly controlled. The form of attentional manipulation by Baldassi and Simoncini may have allowed a boost of reward effects through attention, despite the conclusion of the study (Baldassi and Simoncini, 2011). Secondly, in both Baldassi et al. (2011) and Bayer et al. (2017) reward cues were predictive of the delivery of reward during the main task, whereas reward cues in our task were previously associated with reward value and did not lead to rewards anymore. Reward predicting incentives can overall exert stronger effects on perception as better perceptual performance is constantly reinforced by the delivery of reward. This however does not allow to study the pure role of 
the associative value independent of any task-related feedback or continuous reward reinforcement. However, in light of these studies and the fact that the reward effects on their own were weak in our paradigm, we surmise that independent effects of attention and reward can only be observed under conditions where reward can exert its maximal influence on perception without any assistance from attentional mechanisms. In other contexts, such as ours where rewards are learned prior to the task and are not further reinforced, associative value of stimuli may affect perception primarily through modulating attention.

An important novel aspect of the current study is to investigate effects of visual and auditory cues that were previously associated with high monetary reward outcomes. Previous studies on value-driven mechanisms have been mostly focused on reward effects in the visual modality (for review see: Failing and Theeuwes, 2018), although reward effects have also been reported in other sensory modalities (Goltstein et al., 2013; Pleger et al., 2008; Rutkowski and Weinberger, 2005; Shuler and Bear, 2006; Stanisor et al., 2013; Weil et al., 2010). This leaves a question of the extent to which value-driven mechanisms reflect a general principle of information processing across sensory modalities. A few recent studies approached this question by testing cross-modal reward modulations (Anderson, 2016a; Pooresmaeili et al., 2014; Sanz et al., 2018), testing the competition of reward and attention in other sensory modalities (Anderson et al., 2011b; Asutay and Västfjäll, 2016; Baines et al., 2011; Bourgeois et al., 2016; Chelazzi et al., 2014; Failing et al., 2015; Kim et al., 2021; Qin et al., 2021; Tankelevitch et al., 2020; Yantis et al., 2012), and comparing intra- and cross-modal reward modulation directly (Anderson, 2016a; Bean et al., 2021; Bruns et al., 2014; Cheng et al., 2020; Kang et al., 2017; Pooresmaeili et al., 2014; Vakhrushev et al., 2021). The emerging picture from these studies is that value-driven mechanisms reflect a general information processing principle that operates across modalities.

One important principle across sensory modalities is that when distractors or taskirrelevant features are associated with high reward they may potentially interfere with the processing of the target (for review see: Anderson et al., 2021). In line with this, recent studies (Vakhrushev et al., 2021) showed a suppression of visual sensitivity when the taskirrelevant reward cue was in the visual modality (i.e., intra-modally), but surprisingly visual sensitivity was enhanced when reward was cued through the auditory modality (e.g., Pooresmaeili et al., 2014; Vakhrushev et al., 2021). Hence, reward information exerted different effects on sensory processing dependent on whether it was cued intra- or crossmodally suggesting that modality-specific attentional resources might contribute to valuedriven effects. Interestingly, although we used a different task and explicitly controlled the 
amount of spatial attention towards reward cues, our results are in line with the interpretation provided by (Vakhrushev et al., 2021). We found almost no effect of visual reward value in the main task when reward was only cued through vision (i.e., VHSN), but instead there was a dominance of auditory reward value. This was despite the fact that reward value of both modalities was successfully learned and influenced behavior during the conditioning. Notably, although visual high-value cues on their own (i.e., in VHSN configuration) led to almost indistinguishable ERPs relative to a no-value condition (VNSN), joint presence of high value in both modalities (i.e., VHSH) enhanced the ERPs, especially in case of N1, above and beyond both VNSN and VNSH conditions, indicating that congruent reward in both sensory modalities strengthens reward-driven modulation of attention. These two findings reveal two important stages of reward-driven modulation of sensory processing. The first stage occurs locally within each sensory modality. At this stage, reward information competes with attention in order to gain priority in sensory processing (Anderson et al., 2021, 2011a; Failing and Theeuwes, 2018). As reward information conveyed through vision could potentially compete with the change detection task, for instance by withdrawing resources from a relevant feature (luminance) and allocating them to an irrelevant feature (line orientation), the putative local competition of reward and attention can lead to a reduction of reward effects. However, the local competition can be overturned by rewards in another sensory modality, suggesting that at a second stage, the reward information integrates across different sensory modalities (Bruns et al., 2014; Cheng et al., 2020; Wise, 2004) resulting in an additive effect of reward of two sensory modalities and general improvement of sensory representations, as found when comparing VHSH against VHSN and VNSH conditions. Future studies will be needed to test the validity of this proposal.

We note that the lack of reward effects in visual modality could reflect two potential causes: (1) As mentioned above, these results may indicate a potential interference of reward information in visual modality with the specificity of the change detection task. Alternatively, (2) participants may have actually relied more strongly on the auditory modality when detecting a change, hence processing auditory reward values better than visual reward values. The second cause is less likely to account for our results since during the conditioning phase, reward effects were present in both modalities, and participants managed to detect changes in visual stimuli in more than $80 \%$ of all trials. Based on a significant prolongation of response times in VHSN configuration compared to both VNSH and VHSH, as well as lack of any difference between evoked responses to VNSN and VHSH, we surmise that the first scenario, i.e., modality-specific limitations induced by 
competition between attentional and reward signals; resulted in dampening of reward effects in visual modality. Nevertheless, both scenarios point to the fact that our effects may depend on the specific change detection task that was employed and future studies will be needed to test their generalizability to other contexts.

Finally, in our experiment, participants learned the reward value of each sensory modality separately and then were exposed to bimodal stimuli containing the same or different reward values in two modalities. The pattern of results observed here may be due to this training protocol, as auditory and visual cues were never paired during the learning. Alternatively, learning the associatve value of a bimodal stimulus may promote integrating sensory features and reward values more strongly across sensory modalities and thereby lead to reward modulations that have less dependence on modality-specific mechanisms. Future studies will be needed to examine the role of learning protocols in how reward signals from different sensory modalities interact.

\section{Conclusion}

In summary, we found that the allocation of spatial attention towards audiovisual stimuli is guided by the associative reward value of auditory and visual modalities. In the context of the task employed here, value-driven modulation of attention was more robust in auditory modality, with the maximum effect observed when both visual and auditory components of an audiovisual stimulus were associated with high value. These results inspire a two-stage model in which reward information is first represented separately in each sensory modality and is subsequently integrated across modalities. The integration of reward value boosts the combined associative value of a bimodal stimulus and at the same time enhances the attentional selection of the task-relevant information. 


\subsection{References}

Alais, D., Morrone, C., Burr, D., 2006. Separate attentional resources for vision and audition. Proc. R. Soc. B Biol. Sci. 273, 1339-1345. https://doi.org/10.1098/rspb.2005.3420

Algazi, U., Duda, R., Thompson, D., The, C.A.-2001 I.W. on, 2001. The CIPIC HRTF Database, in Applications of Signal Processing to Audio and Acoustics. IEEE Work. on the, 99-102.

Anderson, B.A., 2016a. Value-driven attentional capture in the auditory domain. Attention, Perception, Psychophys. 78, 242-250. https://doi.org/10.3758/s13414-015-1001-7

Anderson, B.A., 2016b. The attention habit: how reward learning shapes attentional selection. Ann. N. Y. Acad. Sci. 1369, 24-39. https://doi.org/10.1111/nyas.12957

Anderson, B.A., 2013. A value-driven mechanism of attentional selection. J. Vis. 13, 7-7. https://doi.org/10.1167/13.3.7

Anderson, B.A., Kim, H., Kim, A.J., Liao, M.-R., Mrkonja, L., Clement, A., Grégoire, L., 2021. The Past, Present, and Future of Selection History. Neurosci. Biobehav. Rev. 130, 326-350. https://doi.org/10.1016/j.neubiorev.2021.09.004

Anderson, B.A., Laurent, P.A., Yantis, S., 2011a. Learned Value Magnifies Salience-Based Attentional Capture. PLoS One 6, e27926. https://doi.org/10.1371/journal.pone.0027926

Anderson, B.A., Laurent, P.A., Yantis, S., 2011b. Value-driven attentional capture. Proc. Natl. Acad. Sci. U. S. A. 108, 10367-10371. https://doi.org/10.1073/pnas.1104047108

Asutay, E., Västfjäll, D., 2016. Auditory attentional selection is biased by reward cues. Sci. Rep. 6, 1-6. https://doi.org/10.1038/srep36989

Baines, S., Ruz, M., Rao, A., Denison, R., Nobre, A.C., 2011. Modulation of neural activity by motivational and spatial biases. Neuropsychologia 49, 2489-2497. https://doi.org/10.1016/j.neuropsychologia.2011.04.029

Baldassi, S., Simoncini, C., 2011. Reward sharpens orientation coding independently of attention. Front. Neurosci. 5, 13. https://doi.org/10.3389/fnins.2011.00013

Bayer, M., Rossi, V., Vanlessen, N., Grass, A., Schacht, A., Pourtois, G., 2017. Independent effects of motivation and spatial attention in the human visual cortex. Soc. Cogn. Affect. Neurosci. 12, 146-156. https://doi.org/10.1093/scan/nsw162

Bean, N.L., Stein, B.E., Rowland, B.A., 2021. Stimulus value gates multisensory integration. Eur. J. Neurosci. 53, 3142-3159. https://doi.org/10.1111/ejn.15167

Bourgeois, A., Chelazzi, L., Vuilleumier, P., 2016. How motivation and reward learning modulate selective attention. Prog. Brain Res. 229, 325-342. https://doi.org/10.1016/BS.PBR.2016.06.004

Brainard, D.H., 1997. The Psychophysics Toolbox. Spat. Vis. 10, 433-436. https://doi.org/10.1163/156856897X00357

Bruns, P., Maiworm, M., Röder, B., 2014. Reward expectation influences audiovisual spatial integration. Attention, Perception, Psychophys. 76, 1815-1827. https://doi.org/10.3758/s13414-014-0699-y

Carrasco, M., 2011. Visual attention: The past 25 years. Vision Res. 51, 1484-1525. https://doi.org/10.1016/J.VISRES.2011.04.012

Chelazzi, L., Eštočinová, J., Calletti, R., Gerfo, E. Lo, Sani, I., Libera, C. Della, Santandrea, E., 2014. Altering spatial priority maps via reward-based learning. J. Neurosci. 34, 8594-8604. https://doi.org/10.1523/JNEUROSCI.0277-14.2014

Chelazzi, L., Perlato, A., Santandrea, E., Della Libera, C., 2013. Rewards teach visual selective attention. Vision Res. 85, 58-72. https://doi.org/10.1016/J.VISRES.2012.12.005

Cheng, Saglam, A., André, S., Pooresmaeili, A., 2020. Cross-Modal Integration of Reward Value during Oculomotor Planning. eneuro 7, ENEURO.0381-19.2020. https://doi.org/10.1523/ENEURO.0381-19.2020

Della Libera, C., Chelazzi, L., 2009. Learning to Attend and to Ignore Is a Matter of Gains and Losses. Psychol. Sci. 20, 778-784. https://doi.org/10.1111/j.1467-9280.2009.02360.x

Della Libera, C., Chelazzi, L., 2006. Visual Selective Attention and the Effects of Monetary Rewards. Psychol. Sci. 17, 222-227. https://doi.org/10.1111/j.1467-9280.2006.01689.x

Delorme, A., Makeig, S., 2004. EEGLAB: An open source toolbox for analysis of single-trial EEG dynamics including independent component analysis. J. Neurosci. Methods. 
https://doi.org/10.1016/j.jneumeth.2003.10.009

Desimone, R., Duncan, J., 1995. NEURAL MECHANISMS OF SELECTIVE VISUAL ATTENTION, Annu. Rev. Neurosci.

Duncan, J., Martens, S., Ward, R., 1997. Restricted attentional capacity within but not between sensory modalities. Nature 387, 808-810. https://doi.org/10.1038/42947

Failing, M., Nissens, T., Pearson, D., Le Pelley, M., Theeuwes, J., 2015. Oculomotor capture by stimuli that signal the availability of reward. J. Neurophysiol. 114, 2316-2327. https://doi.org/10.1152/jn.00441.2015

Failing, M., Theeuwes, J., 2018. Selection history: How reward modulates selectivity of visual attention. Psychon. Bull. Rev. 25, 514-538. https://doi.org/10.3758/s13423-017-1380-y

Garcia-Lazaro, H.G., Bartsch, M. V., Boehler, C.N., Krebs, R.M., Donohue, S.E., Harris, J.A., Schoenfeld, M.A., Hopf, J.M., 2018. Dissociating reward- and attention-driven biasing of global feature-based selection in human visual cortex. J. Cogn. Neurosci. 31, 469-481. https://doi.org/10.1162/jocn_a_01356

Goltstein, P.M., Coffey, E.B.J., Roelfsema, P.R., Pennartz, C.M.A., 2013. In vivo two-photon Ca2+ imaging reveals selective reward effects on stimulus-specific assemblies in mouse visual cortex. J. Neurosci. 33, 11540-11555. https://doi.org/10.1523/JNEUROSCI.1341-12.2013

Hickey, C., Chelazzi, L., Theeuwes, J., 2010. Reward changes salience in human vision via the anterior cingulate. J. Neurosci. 30, 11096-11103. https://doi.org/10.1523/JNEUROSCI.102610.2010

Hughes, G., Mathan, S., Yeung, N., 2013. EEG indices of reward motivation and target detectability in a rapid visual detection task. Neuroimage 64, 590-600. https://doi.org/10.1016/j.neuroimage.2012.09.003

Kang, G., Wang, L., Zhou, X., 2017. Reward interacts with modality shift to reduce cross-modal conflict. J. Vis. 17. https://doi.org/10.1167/17.1.19

Kim, A.J., Lee, D.S., Anderson, B.A., 2021. Previously reward-associated sounds interfere with goaldirected auditory processing. Q. J. Exp. Psychol. 74, 1257-1263. https://doi.org/10.1177/1747021821990033

Kiss, M., Driver, J., Eimer, M., 2009. Reward Priority of Visual Target Singletons Modulates EventRelated Potential Signatures of Attentional Selection. Psychol. Sci. 20, 245-251. https://doi.org/10.1111/j.1467-9280.2009.02281.x

Krebs, R.M., Boehler, C.N., Woldorff, M.G., 2010. The influence of reward associations on conflict processing in the Stroop task. Cognition 117, 341-347. https://doi.org/10.1016/j.cognition.2010.08.018

Le Pelley, M.E., Mitchell, C.J., Beesley, T., George, D.N., Wills, A.J., 2016. Attention and associative learning in humans: An integrative review. Psychol. Bull. 142, 1111-1140. https://doi.org/10.1037/bul0000064

Leo, F., Noppeney, U., 2014. Conditioned Sounds Enhance Visual Processing. PLoS One 9, e106860. https://doi.org/10.1371/journal.pone.0106860

Maunsell, J.H.R., 2004. Neuronal representations of cognitive state: Reward or attention? Trends Cogn. Sci. 8, 261-265. https://doi.org/10.1016/j.tics.2004.04.003

Mognon, A., Jovicich, J., Bruzzone, L., Buiatti, M., 1994. ADJUST: An automatic EEG artifact detector based on the joint use of spatial and temporal features. Psychophysiology. https://doi.org/10.1111/j.1469-8986.2010.01061.x

Pessoa, L., 2015. Multiple influences of reward on perception and attention. Vis. cogn. 23, 272-290. https://doi.org/10.1080/13506285.2014.974729

Pfabigan, D.M., Seidel, E.M., Sladky, R., Hahn, A., Paul, K., Grahl, A., Küblböck, M., Kraus, C., Hummer, A., Kranz, G.S., Windischberger, C., Lanzenberger, R., Lamm, C., 2014. P300 amplitude variation is related to ventral striatum BOLD response during gain and loss anticipation: An EEG and fMRI experiment. Neuroimage 96, 12-21. https://doi.org/10.1016/j.neuroimage.2014.03.077

Pleger, B., Blankenburg, F., Ruff, C.C., Driver, J., Dolan, R.J., 2008. Reward facilitates tactile judgments and modulates hemodynamic responses in human primary somatosensory cortex. J. Neurosci. 28, 8161-8168. https://doi.org/10.1523/JNEUROSCI.1093-08.2008

Pooresmaeili, A., FitzGerald, T.H.B., Bach, D.R., Toelch, U., Ostendorf, F., Dolan, R.J., 2014. Cross-modal effects of value on perceptual acuity and stimulus encoding. Proc. Natl. Acad. Sci. 
U. S. A. 111, 15244-15249. https://doi.org/10.1073/pnas.1408873111

Qin, N., Gu, R., Xue, J., Chen, C., Zhang, M., 2021. Reward-driven attention alters perceived salience. J. Vis. 21, 1-13. https://doi.org/10.1167/JOV.21.1.7

Raymond, J.E., O’Brien, J.L., 2009. Selective visual attention and motivation: the consequences of value learning in an attentional blink task. Psychol. Sci. 20, 981-8. https://doi.org/10.1111/j.1467-9280.2009.02391.x

Rutkowski, R.G., Weinberger, N.M., 2005. Encoding of learned importance of sound by magnitude of representational area in primary auditory cortex. Proc. Natl. Acad. Sci. U. S. A. 102, 13664 13669. https://doi.org/10.1073/pnas.0506838102

San Martín, R., Appelbaum, L.G., Huettel, S.A., Woldorff, M.G., 2016. Cortical Brain Activity Reflecting Attentional Biasing Toward Reward-Predicting Cues Covaries with Economic Decision-Making Performance. Cereb. Cortex 26, 1-11. https://doi.org/10.1093/cercor/bhu 160

Sanz, L.R.D., Vuilleumier, P., Bourgeois, A., 2018. Cross-modal integration during value-driven attentional capture. $\quad$ Neuropsychologia $120, \quad 105-112$. https://doi.org/10.1016/j.neuropsychologia.2018.10.014

Serences, J.T., 2008. Value-Based Modulations in Human Visual Cortex. Neuron 60, 1169-1181. https://doi.org/10.1016/j.neuron.2008.10.051

Shuler, M.G., Bear, M.F., 2006. Reward timing in the primary visual cortex. Science (80-. ). 311, 1606-1609. https://doi.org/10.1126/science.1123513

Stanisor, L., Van Der Togt, C., Pennartz, C.M.A., Roelfsema, P.R., 2013. A unified selection signal for attention and reward in primary visual cortex. Proc. Natl. Acad. Sci. U. S. A. 110, 91369141. https://doi.org/10.1073/pnas.1300117110

Talsma, D., Woldorff, M.G., 2005. Selective attention and multisensory integration: Multiple phases of effects on the evoked brain activity. J. Cogn. Neurosci. 17, 1098-1114. https://doi.org/10.1162/0898929054475172

Tankelevitch, L., Spaak, E., Rushworth, M.F.S., Stokes, M.G., 2020. Previously Reward-Associated Stimuli Capture Spatial Attention in the Absence of Changes in the Corresponding Sensory Representations as Measured with MEG. J. Neurosci. 40, 5033-5050. https://doi.org/10.1523/JNEUROSCI.1172-19.2020

Vakhrushev, R., Cheng, F., Schacht, A., Pooresmaeili, A., 2021. Differential effects of intra-modal and cross-modal reward value on visual perception: ERP evidence. bioRxiv 2021.09.29.462374. https://doi.org/10.1101/2021.09.29.462374

Watson, A.B., Pelli, D.G., 1983. Quest: A Bayesian adaptive psychometric method. Percept. Psychophys. 33, 113-120. https://doi.org/10.3758/BF03202828

Weil, R.S., Furl, N., Ruff, C.C., Symmonds, M., Flandin, G., Dolan, R.J., Driver, J., Rees, G., 2010. Rewarding Feedback After Correct Visual Discriminations Has Both General and Specific Influences on Visual Cortex. J. Neurophysiol. 104, 1746-1757. https://doi.org/10.1152/jn.00870.2009

Wise, R.A., 2004. Dopamine, learning and motivation. Nat. Rev. Neurosci. https://doi.org/10.1038/nrn1406

Woldorff, M.G., 1993. Distortion of ERP averages due to overlap from temporally adjacent ERPs: Analysis and correction. Psychophysiology 30, 98-119. https://doi.org/10.1111/j.14698986.1993.tb03209.x

Yantis, S., Anderson, B.A., Wampler, E.K., Laurent, P.A., 2012. Reward and attentional control in visual search. Nebraska Symp. Motiv. https://doi.org/10.1007/978-1-4614-4794-8-5 


\section{Chapter 5: General discussion}

\subsection{Summary of the results}

The goal of the present $\mathrm{PhD}$ project was to gain insight into the underlying mechanisms of reward-driven modulation of sensory perception. Specifically, the project aimed to determine how intra- and cross-modal reward cues influence early sensory processing (Objective 1) and whether effects of these intra- and cross-modal reward cues interact with selective attention (Objective 2). To pursue these aims, three studies were conducted: in Study 1, visual and auditory cues were first associated with high- and low-value monetary reward outcomes, and later, during the post-conditioning phase, effects of reward cues were compared across modality conditions. During the post-conditioning phase, participants performed a visual orientation discrimination task in the presence of task-irrelevant visual (intra-modal) and auditory (cross-modal) reward cues. During the conditioning phase, when reward cues were the target of the task, reward value in both modalities enhanced the amplitude of the P300 component over posterior electrodes. The analysis of latency of the $\mathrm{N} 1$ peak over posterior electrodes revealed that high-value cues sped up the peak onset in visual condition. In contrast, during the post-conditioning phase, when reward cues were irrelevant to the task, the analysis of behavioral results revealed an interaction between the modality of the reward cue and its value. Cross-modal reward cues enhanced visual sensitivity, whereas intra-modal reward cues tended to suppress it. An analysis of P1 and N1 peak amplitudes over the posterior electrodes revealed a similar interaction pattern as shown in behavioral results. Intra-modal reward cues emerged earlier and resulted in a suppression of the P1 peak amplitude (90-120 ms). Cross-modal high reward cues instead led to an enhanced positivity emerging later starting at the $\mathrm{N} 1$ window $(180-250 \mathrm{~ms})$, suggesting that intra- and cross-modal reward modulations elicit different effects on visual sensory processing. Taken together, the results demonstrated value-driven modulation at multiple stages during visual sensory processing: intra-modally, where competition between value, salience, and goal-directed signals resulted in the interference between them occurring very early (90-120 ms) (Anderson, 2013; Anderson et al., 2021; Awh et al., 2012), and crossmodally, when reward signals from other modalities impacted the later processing of sensory signals presumably through feedback signals from multimodal areas (Anderson, 2016a; Pooresmaeili et al., 2014). At a post-perceptual stage coinciding with P300 window, anticipation of the reward delivery in the conditioning phase led to similar reward 
modulations in both modalities, suggesting that at this stage reward effects occur independent of the sensory properties of reward cues (Goldstein et al., 2006; Luque et al., 2017, 2015; Pornpattananangkul and Nusslock, 2015; Yeung and Sanfey, 2004). Yet, it was unclear whether the differential reward effects were due to differences in how visual and auditory reward cues integrate with the target or in how they affect attentional mechanisms.

Study 2 tried to pit these possibilities, attentional versus object integration accounts, against each other and compared behavioral effects of intra- and cross-modal reward cues in terms of the spatiotemporal characteristics of reward modulations. Specifically, Study 2 focused only on behavioral indices while manipulating the focus of spatial attention (experiment 1) and the temporal (experiment 2) arrangement between reward and target cues. The focus of attention was changed by narrowing or widening it with small, medium, and large frames that appeared at the beginning of each trial (Castiello and Umiltà, 1992). Similar to Study 1, we found a difference between intra- and cross-modal reward modulations: intra-modal reward cues sped up responses, but only when the attention focused narrowly on the target. Cross-modal reward cues, on the other hand, improved visual sensitivity, but only when attentional focus was wide. Experiment 2 tested reward effects at a wide focus of attention and manipulated only the asynchrony between reward cues and the target. If reported effects in Study 1 resulted from how rewards are integrated with the target, reward modulations were expected to be largest at asynchrony $=0$, i.e., when reward cues and the target were synchronized and hence grouped with each other. Additionally, if perceptual competition played a significant role in suppression effects showed in intra-modal condition, preceding reward cues should enhance the processing of visual targets similarly to cross-modal reward cues. The results revealed reward effects both for intra- and crossmodal conditions only when reward and target cues were synchronized, suggesting that the differential effects of intra-modal and cross-modal cues is related to how they are integrated with the target. As we also found a dependence of reward effects on the size of attentional focus these results suggest that a combination of attentional priming and a change in grouping/integration account for our effects in Study 1 and Study 2.

To directly assess the interaction of reward and attention, Study 3 specifically manipulated the allocation of spatial attention towards reward cues from both modalities (Objective 2). The experimental task was slightly different from study 1 and study 2 . Here, conditioning and post-conditioning phases used a similar change detection task. A change was a brief interruption of the peripheral bimodal audio-visual cue during its presentation. During the post-conditioning phase, participants were instructed to report the change only 
on the attended side (Talsma and Woldorff, 2005) and ignore cues on the unattended side. Importantly, here we tried to make the stimulus conditions as similar as possible to each other in terms of their sensory properties, thus using bimodal audiovisual stimuli across reward conditions. There were four conditions, two of which were bimodal cues with both modalities associated with either high- or no-rewards, and the other two were bimodal cues with only one modality associated with high reward and the other associated with no reward. To compare effects across reward conditions when these cues were attended and unattended, the study relied on electrophysiological measurements, where only cues without a change were analyzed. The results revealed an interaction between reward and attention. Specifically, reward modulated the intensity of attentional modulation. High-value visual and auditory cues produced higher P1 and N1 amplitudes when they were attended and lower P1 and N1 amplitudes when unattended, compared to no-value cues. Since reward cues modulated ERP components only through attention, the following analysis focused on the reward effects on attention. When reward effects across visual and auditory modalities were compared directly, an interaction effect was found corresponding to a stronger modulation of attention by auditory rewards. Although associating only the visual modality with reward led to no significant modulation of attention compared to no-reward condition, reward signals in both modalities enhanced responses above and beyond modulations elicited by rewards in either of the modalities. This result once again supports the idea that reward modulation occurs at multiple stages during sensory processing and can be boosted by combining reward-driven effects across modalities. In the following section, the results of these three studies will be discussed in a more general context.

\subsection{Intra- and cross-modal reward effects on behavioral and electrophysiological indices}

Overall, this dissertation provides evidence that both intra- and cross-modal reward cues could modulate behavioral and electrophysiological indices even when they are irrelevant to the task and do not deliver reward outcomes (Studies 1, 2, and 3). These results are consistent with one that was reported in the literature, where similar reward effects were reported separately for intra- (Anderson, 2013; for review see: Anderson et al., 2021; Della Libera and Chelazzi, 2006; Hickey et al., 2010b; Kim et al., 2021; Yantis et al., 2012) and cross-modal (Anderson, 2016a; Pooresmaeili et al., 2014) reward cues. However, in the present dissertation, intra- and cross-modal reward effects were compared directly, which was not yet reported in the past literature. 
A comparison of reward effects across intra- and cross-modal reward conditions revealed a differential pattern of reward-related modulation. Specifically, cross-modal reward cues (Study 1, 2, Study 3) improved behavioral measures of visual sensitivity for high-value compared to low-value cross-modal cues and led to a later enhancement of the ERP amplitudes starting at the N1 window. These results are in line with the previous behavioral and neurophysiological studies that report positive effects of cross-modal highvalue cues on behavior and neuronal responses (Bean et al., 2021; Bruns et al., 2014; Leo and Noppeney, 2014; Pooresmaeili et al., 2014).

We observed a different pattern of results for intra-modal rewards in Study 1 and Study 2 whereas they were expected to have similar effects to cross-modal rewards that enhanced behavioral and electrophysiological indices. This expectation was based on previous findings (Stanisor et al., 2013) where reward boosted processing of stimuli that belonged to the same object (Duncan, 1984; Pooresmaeili and Roelfsema, 2014). Therefore Study 1 and Study 2 positioned the target and reward cues in close proximity or even overlapping with each other. Contrary to this prediction, intra-modal reward cues interfered with the sensory processing of visual target, which resulted in the decrease of visual sensitivity and suppression of early ERP responses (90-120 ms) by a high- compared to low-value cues. Despite this, literature has support for the interference effects. Previously it has been shown that when a visual reward cue appears as a distractor, it impairs behavioral responses towards the target cue, a mechanism is also known as value-driven attentional capture (Anderson et al., 2013, for review see: 2021, 2011a; Chang and Egeth, 2021; Cheng et al., 2020; Hickey et al., 2009; Itthipuripat et al., 2019; Pearson et al., 2020; Tankelevitch et al., 2019). However, despite being at the same location, the intra-modal reward cue, i.e. a color, captured attention to a feature different from the one that was relevant to the task, i.e. orientation, hence interfering with target processing. Study 2 tested whether the perceptual arrangement of the reward cues and the target could account for these differences. The results showed that intra-modal high-value cues facilitated response times only when the reward cue was allocated narrowly with the target cue. The results showed that competition between features could not fully explain suppression effects in Study 1. Instead, intra-modal and cross-modal reward cues each can exert facilitatory effects if they are presented at the optimal spatial arrangement relative to the target. Study 3 further tried to improve some of the shortcomings of Study 1 and 2 by employing bimodal stimuli and additionally by making the reward cues less incongruent with the goal-directed requirements of the task. However, the results revealed again a difference between reward effects cued through visual modality 
compared to auditory modality. Importantly, this mechanism was evident only when visual intra-modal reward cues competed for sensory processing, either with goal-directed visual (Study 1 and Study 2) or auditory (Study 3) cues. This may suggest that the reduction of neuronal responses by reward may be a general characteristic of reward-related modulations occurring due to direct dopaminergic inputs to the visual cortex (Arsenault et al., 2013) or value-driven attentional gain control mechanisms (Shapcott et al., 2020) that adjust signal to noise ratio of neuronal responses. However, auditory reward effects seem to engage different mechanisms, likely due to the engagement of multimodal areas such as the Superior Temporal Cortex (Pooresmaeili et al., 2014).

Additional insight can be gained by comparing our ERP results against the previous literature. Specifically, study 1 and Study 3 compared electrophysiological indices of intraand cross-modal reward modulations. Previous findings have reported early reward-driven modulations separately for auditory (Anderson, 2016a; Asutay and Västfjäll, 2016; Kim et al., 2021; Pooresmaeili et al., 2014; Wikman et al., 2019) and especially for visual modalities (Bayer et al., 2017; Hickey et al., 2010b; Luque et al., 2017). For example, it has been shown that that visual reward cues affect C1, P1, and N1 ERP components that are related to early sensory processing P1 (Bachman et al., 2020; Baines et al., 2011; Bayer et al., 2017; Hammerschmidt et al., 2018; Hickey et al., 2010b; Luque et al., 2017) and ERP components related to late reward evaluation P300 and LPC (Bayer et al., 2017; Hughes et al., 2013; J. Liu et al., 2020; Novak and Foti, 2015; Rossi et al., 2017). Here, for the first time we investigated the ERP signature of reward signaled through either visual or auditory modalities and showed important difference in the timing (Study 1) and amplitude (Study 3) of neuronal responses elicited by each sensory modality. Follow-up studies are necessary to understand mechanisms behind the differential effects of intra- and cross-modal rewards that were observed here.

\subsection{Interaction of reward effects with selective attention}

An interaction between reward and attentional mechanisms has been debated for a long time (Della Libera and Chelazzi, 2009, 2006; Kim et al., 2021; Maunsell, 2004; for review see: Pessoa, 2015). In line with this, Study 1 showed interaction effects between value and modality, where intra-modal high-value cues suppressed visual sensitivity and the peak of P1 component. On another side, cross-modal reward cues exert opposite effects (Pooresmaeili et al., 2014). This study raised several questions about the role of attention, specifically whether intra-modal reward effects could be explained with modulation of 
attention (Maunsell, 2004; Pessoa, 2015) and what is the role of modality-specific attentional resources for intra- and cross-modal reward modulation (Driver and Noesselt, 2008; Duncan et al., 1997). The following Study 2 explored these questions by modulating spatial (experiment 1) and temporal (experiment 2) priming of attention. Experiment 1 showed an interaction between attention and intra- and cross-modal reward modulation, showing that modality-specific attentional resources interact with reward modulation. However, experiment 2 revealed cross-modal reward effects only when cues were synchronized. Hence, measured reward effects depended on cross-modal integration (Bean et al., 2021; Bruns, 2019) and did not affect the strength of the attentional priming. Study 3 compared intra- and cross-modal reward effects with attention directly. We showed an interaction between attention and reward effects, where reward cues modulated ERP components by enhancing attentional control (Kang et al., 2017; Padmala and Pessoa, 2010; Pearson et al., 2020; Pessoa and Engelmann, 2010; Pool et al., 2014). Similar to previous findings in Study 1 and Study 2, we also found an interaction between sensory modality and reward value, as visual rewards, unlike auditory reward, did not modulate attention on their own. However, when both cues of the audiovisual stimulus had high reward, robust reward modulations were found. These two findings reveal two important stages of reward-driven modulation of sensory processing. The first stage occurs locally within each sensory modality. At this stage, reward information competes with attention to prioritize sensory processing (Anderson et al., 2021, 2011a; Failing and Theeuwes, 2018). As reward information conveyed through vision could potentially compete with the change detection task, for instance by withdrawing resources from a relevant feature (luminance) and allocating them to an irrelevant feature (line orientation), the putative local competition of reward and attention can lead to a reduction of reward effects. However, the local competition can be overturned by rewards in another sensory modality, suggesting that at a second stage, the reward information integrates across different sensory modalities (Bruns et al., 2014; Cheng et al., 2020; Wise, 2004), resulting in additive reward effects between visual and auditory sensory modalities and general improvement of sensory representations.

\subsection{Embedding intra- and cross-modal reward effects in cognitive models of perception}

In general, our results suggested that intra- and cross-modal reward modulations occur at different stages of sensory information processing where the first stage (intra-modal) and second stage (downstream effects from other sensory modalities) rely on different perceptual 
mechanisms. The suggested two-stage model is an extension of current models of rewarddriven modulation of perception (Anderson et al., 2021; for review see: Failing and Theeuwes, 2018; Pessoa, 2015) and specifically is related to the 'priority map' model explained in the introduction (Bisley, 2011; Wolfe, 2021; Zelinsky and Bisley, 2015). The key extensions to this model provided by the current thesis are as follows. Previous studies primarily related the reward effects to the priority map using visual tasks and especially visual search tasks (Failing and Theeuwes, 2018; Wolfe, 2021). In these tasks, at any given time, an observer first evaluates location values across currently available visual locations and then makes a saccade to a location with the highest importance, salience, or importance (Awh et al., 2012; Itti and Koch, 2001). Using such paradigms, it was suggested that valuedriven attention similar to mechanisms of stimulus-driven attention and goal-driven attention, appears as an independent mechanism that competes for processing resources when the observer chooses the next target location (Anderson, 2016b, 2013; Anderson et al., 2021; Awh et al., 2012). Recent studies supported this model by showing similar competition effects when reward effects were measured in other modalities (Kim et al., 2021) and even cross-modally (Anderson, 2016a). Despite this, some studies contradicted this model and showed generally positive reward effects when these effects were measured cross-modally (Bean et al., 2021; Bruns et al., 2014; Cheng et al., 2020; Kang et al., 2018; Leo and Noppeney, 2014; Pooresmaeili et al., 2014). To understand these divergent effects the current study compared cross-modal and intra-modal effects directly.

Our results not only extend the priority map model to the domain of auditory/crossmodal reward value, but also provide important insights on how the architecture of such a model should be revised to accommodate reward modulations. Specifically, we showed that reward effects emerge in multiple stages of information processing. At the first stage, as depicted with the 'priority map' model, reward signals compete for processing resources thus impairing performance when task-irrelevant. This competition occurs in the early sensory areas (Arsenault et al., 2013; Bao et al., 2001; Hickey et al., 2010b; Hollerman and Schultz, 1998; Schacht et al., 2012; Serences, 2008) before down-stream effects take place. At the second stage, reward signals are sent directly across sensory modalities, thus bypassing the competition between different sensory signals since the processing of these reward signals relies on the modality-specific attentional resources. This conclusion was based on systematic results that emerged when reward effects were compared between crossmodal and intra-modal conditions. A distinct characteristic of the second stage is that the processing of reward signals does not interfere or compete with processing of salient task- 
related targets, resulting in enhancement of target sensory representation. The pathway of this modulatory effect was suggested to go through STS (superior temporal sulcus) (Pooresmaeili et al., 2014) and reflect the enhancement of cross-modal integration (Bean et al., 2021; Bruns et al., 2014; Cheng et al., 2020) by inducing early sensory plasticity in modalities that share reward-related object (Hollerman and Schultz, 1998). This shows that value-driven plasticity in visual perception can be induced not only by signals from the visual modality but also cross-modally. Embedding cross-modal rewards in visual tasks is a promising tool to assist vision, especially in the face of visual impairments, through boosting cross-modal advantages conferred by another intact sensory modality. Further studies are needed to understand specific characteristics of this mechanism.

\subsection{Final conclusions and future perspectives}

The aim of the present $\mathrm{PhD}$ project was to understand underlying mechanisms of reward-driven modulation of sensory perception and its interaction with attention. In three studies we identified the behavioral and neural signatures of reward effects signaled from the visual or auditory modality. We found that task-irrelevant valuable signals impair performance in a goal-directed task when reward is in the same sensory modality. However, when reward signals do not share sensory modality with the goal-directed task, they exert positive effect on behavioral and neuronal indices. These behavioral effects corresponded to a difference in timing of the reward-driven ERP modulations elicited by intra- and crossmodal reward cues, with the latter occurring later. Additionally, we also identified the ERP correlates of reward-driven modulation of spatial attention and their dependence on the sensory modality. These findings inspired a two-stage model of reward processing and its interaction with attention that can further the existing cognitive models such as a priority map model. Future empirical and computational studies will be needed to test the validity of this proposal.

We showed that value-driven plasticity in visual perception can be induced not only by signals from the visual modality but also cross-modally. Enhancement of plasticity by reward signals, especially when they were delivered cross-modally, appears as a potent perceptual mechanism and a promising tool to assist vision, especially in the face of visual impairments. With this in mind, current findings are highly relevant for clinical applications, specifically for the patients with deficits in one of the sensory modalities (Dundon et al., 2015; Làdavas et al., 2020; Maier et al., 2019). For example, by pairing visual stimuli with auditory cues, people with visual field deficits may perform better. If reward delivery could 
facilitate this process even more, it could be an important step in developing rehabilitation training, through boosting cross-modal advantages conferred by another intact sensory modality. Further studies are needed to examine the feasibility of using cross-modal rewards in clinical settings. Furthermore, more profound understanding of the underlying mechanisms of intra- and cross-modal reward modulations can be gained by using recording techniques that have higher spatial and temporal resolution which can further assist the future clinical applications. 


\section{References}

Ainslie, G., 1975. Specious reward: A behavioral theory of impulsiveness and impulse control. Psychol. Bull. 82, 463-496. https://doi.org/10.1037/h0076860

Alais, D., Morrone, C., Burr, D., 2006. Separate attentional resources for vision and audition. Proc. R. Soc. B Biol. Sci. 273, 1339-1345. https://doi.org/10.1098/rspb.2005.3420

Algazi, U., Duda, R., Thompson, D., The, C.A.-2001 I.W. on, 2001. The CIPIC HRTF Database, in Applications of Signal Processing to Audio and Acoustics. IEEE Work. on the, 99-102.

Alsius, A., Navarra, J., Campbell, R., Soto-Faraco, S., 2005. Audiovisual integration of speech falters under high attention demands. Curr. Biol. 15, 839-843. https://doi.org/10.1016/j.cub.2005.03.046

Anderson, B.A., 2019. Neurobiology of value-driven attention. Curr. Opin. Psychol. https://doi.org/10.1016/j.copsyc.2018.11.004

Anderson, B.A., 2017. Reward processing in the value-driven attention network: reward signals tracking cue identity and location. Soc. Cogn. Affect. Neurosci. 12, 461-467. https://doi.org/10.1093/scan/nsw141

Anderson, B.A., 2016a. Value-driven attentional capture in the auditory domain. Attention, Perception, Psychophys. 78, 242-250. https://doi.org/10.3758/s13414-015-1001-7

Anderson, B.A., 2016b. The attention habit: how reward learning shapes attentional selection. Ann. N. Y. Acad. Sci. 1369, 24-39. https://doi.org/10.1111/nyas.12957

Anderson, B.A., 2013. A value-driven mechanism of attentional selection. J. Vis. 13, 7-7. https://doi.org/10.1167/13.3.7

Anderson, B.A., Kim, A.J., 2020. Selection history-driven signal suppression. Vis. cogn. https://doi.org/10.1080/13506285.2020.1727599

Anderson, B.A., Kim, H., Kim, A.J., Liao, M.-R., Mrkonja, L., Clement, A., Grégoire, L., 2021. The Past, Present, and Future of Selection History. Neurosci. Biobehav. Rev. 130, 326-350. https://doi.org/10.1016/j.neubiorev.2021.09.004

Anderson, B.A., Laurent, P.A., Yantis, S., 2011a. Value-driven attentional capture. Proc. Natl. Acad. Sci. U. S. A. 108, 10367-10371. https://doi.org/10.1073/pnas.1104047108

Anderson, B.A., Laurent, P.A., Yantis, S., 2011b. Learned Value Magnifies Salience-Based Attentional Capture. PLoS One 6, e27926. https://doi.org/10.1371/journal.pone.0027926

Anderson, B.A., Yantis, S., 2013. Persistence of value-driven attentional capture. J. Exp. Psychol. Hum. Percept. Perform. 39, 6-9. https://doi.org/10.1037/a0030860

Anderson, Laurent, Yantis, Anderson, B.A., Laurent, P.A., Yantis, S., 2013. Reward predictions bias attentional selection. Front. Hum. Neurosci. 7, 1-6. https://doi.org/10.3389/fnhum.2013.00262

Arsenault, J.T., Nelissen, K., Jarraya, B., Vanduffel, W., 2013. Dopaminergic Reward Signals Selectively Decrease fMRI Activity in Primate Visual Cortex. Neuron 77, 1174-1186. https://doi.org/10.1016/j.neuron.2013.01.008

Asutay, E., Västfjäll, D., 2016. Auditory attentional selection is biased by reward cues. Sci. Rep. 6, 1-6. https://doi.org/10.1038/srep36989

Awh, E., Belopolsky, A. V., Theeuwes, J., 2012. Top-down versus bottom-up attentional control: A failed theoretical dichotomy. Trends Cogn. Sci. https://doi.org/10.1016/j.tics.2012.06.010

Bachman, M.D., Wang, L., Gamble, M.L., Woldorff, M.G., 2020. Physical Salience and ValueDriven Salience Operate through Different Neural Mechanisms to Enhance Attentional Selection. J. Neurosci. 40, 5455-5464. https://doi.org/10.1523/JNEUROSCI.1198-19.2020

Baines, S., Ruz, M., Rao, A., Denison, R., Nobre, A.C., 2011. Modulation of neural activity by motivational and spatial biases. Neuropsychologia 49, 2489-2497. https://doi.org/10.1016/j.neuropsychologia.2011.04.029

Balan, P.F., Gottlieb, J., 2006. Integration of exogenous input into a dynamic salience map revealed by perturbing attention. J. Neurosci. 26, 9239-9249. https://doi.org/10.1523/JNEUROSCI.1898-06.2006

Baldassi, S., Simoncini, C., 2011. Reward sharpens orientation coding independently of attention. Front. Neurosci. 5, 13. https://doi.org/10.3389/fnins.2011.00013

Bao, S., Chan, V.T., Merzenich, M.M., 2001. Cortical remodelling induced by activity of ventral 
tegmental dopamine neurons. Nature 412, 79-83. https://doi.org/10.1038/35083586

Basten, U., Biele, G., Heekeren, H.R., Fiebach, C.J., 2010. How the brain integrates costs and benefits during decision making. Proc. Natl. Acad. Sci. U. S. A. 107, 21767-21772. https://doi.org/10.1073/pnas.0908104107

Bayer, M., Grass, A., Schacht, A., 2019. Associated valence impacts early visual processing of letter strings: Evidence from ERPs in a cross-modal learning paradigm. Cogn. Affect. Behav. Neurosci. 19, 98-108. https://doi.org/10.3758/s13415-018-00647-2

Bayer, M., Rossi, V., Vanlessen, N., Grass, A., Schacht, A., Pourtois, G., 2017. Independent effects of motivation and spatial attention in the human visual cortex. Soc. Cogn. Affect. Neurosci. 12, 146-156. https://doi.org/10.1093/scan/nsw162

Bayer, M., Sommer, W., Schacht, A., 2012. P1 and beyond: Functional separation of multiple emotion effects in word recognition. Psychophysiology 49, 959-969. https://doi.org/10.1111/j.1469-8986.2012.01381.x

Bean, N.L., Stein, B.E., Rowland, B.A., 2021. Stimulus value gates multisensory integration. Eur. J. Neurosci. 53, 3142-3159. https://doi.org/10.1111/ejn.15167

Berridge, K.C., 2004. Motivation concepts in behavioral neuroscience. Physiol. Behav. 81, 179-209. https://doi.org/10.1016/J.PHYSBEH.2004.02.004

Berridge, K.C., 2000. Reward learning: Reinforcement, incentives, and expectations. Psychol. Learn. Motiv. - Adv. Res. Theory 40, 223-278.

Berridge, K.C., Kringelbach, M.L., 2015. Pleasure Systems in the Brain. Neuron 86, 646-664. https://doi.org/10.1016/J.NEURON.2015.02.018

Berridge, K.C., Kringelbach, M.L., 2008. Affective neuroscience of pleasure: Reward in humans and animals. Psychopharmacology (Berl). https://doi.org/10.1007/s00213-008-1099-6

Berridge, K.C., Robinson, T.E., 1998. What is the role of dopamine in reward: Hedonic impact, reward learning, or incentive salience? Brain Res. Rev. 28, 309-369. https://doi.org/10.1016/S0165-0173(98)00019-8

Bertelson, P., Radeau, M., 1981. Cross-modal bias and perceptual fusion with auditory-visual spatial discordance. Percept. Psychophys. 29, 578-584. https://doi.org/10.3758/BF03207374

Bisley, J.W., 2011. The neural basis of visual attention. J. Physiol. https://doi.org/10.1113/jphysiol.2010.192666

Bolognini, N., Frassinetti, F., Serino, A., Làdavas, E., 2005. "Acoustical vision” of below threshold stimuli: Interaction among spatially converging audiovisual inputs. Exp. Brain Res. 160, 273282. https://doi.org/10.1007/s00221-004-2005-Z

Bourgeois, A., Chelazzi, L., Vuilleumier, P., 2016. How motivation and reward learning modulate selective attention. Prog. Brain Res. 229, 325-342. https://doi.org/10.1016/BS.PBR.2016.06.004

Brainard, D.H., 1997. The Psychophysics Toolbox. Spat. Vis. 10, 433-436. https://doi.org/10.1163/156856897X00357

Braver, T.S., Krug, M.K., Chiew, K.S., Kool, W., Andrew Westbrook, J., Clement, N.J., Alison Adcock, R., Barch, D.M., Botvinick, M.M., Carver, C.S., Cools, R., Custers, R., Dickinson, A., Dweck, C.S., Fishbach, A., Gollwitzer, P.M., Hess, T.M., Isaacowitz, D.M., Mather, M., Murayama, K., Pessoa, L., Samanez-Larkin, G.R., Somerville, L.H., 2014. Mechanisms of motivation-cognition interaction: Challenges and opportunities. Cogn. Affect. Behav. Neurosci. https://doi.org/10.3758/s13415-014-0300-0

Bruns, P., 2019. The Ventriloquist Illusion as a Tool to Study Multisensory Processing: An Update. Front. Integr. Neurosci. https://doi.org/10.3389/fnint.2019.00051

Bruns, P., Maiworm, M., Röder, B., 2014. Reward expectation influences audiovisual spatial integration. Attention, Perception, Psychophys. 76, 1815-1827. https://doi.org/10.3758/s13414-014-0699-y

Busse, L., Roberts, K.C., Crist, R.E., Weissman, D.H., Woldorff, M.G., 2005. The spread of attention across modalities and space in a multisensory object. Proc. Natl. Acad. Sci. U. S. A. 102, 18751-18756. https://doi.org/10.1073/pnas.0507704102

Bustamante, L., Lieder, F., Musslick, S., Shenhav, A., Cohen, J., 2021. Learning to Overexert Cognitive Control in a Stroop Task. Cogn. Affect. Behav. Neurosci. 1-19. https://doi.org/10.3758/s13415-020-00845-x

Calabresi, P., Picconi, B., Tozzi, A., Di Filippo, M., 2007. Dopamine-mediated regulation of 
corticostriatal synaptic plasticity. Trends Neurosci. https://doi.org/10.1016/j.tins.2007.03.001

Calvert, G.A., Thesen, T., 2004. Multisensory integration: Methodological approaches and emerging principles in the human brain. J. Physiol. Paris 98, 191-205. https://doi.org/10.1016/j.jphysparis.2004.03.018

Carrasco, M., 2011. Visual attention: The past 25 years. Vision Res. 51, 1484-1525. https://doi.org/10.1016/J.VISRES.2011.04.012

Castiello, U., Umiltà, C., 1992. Splitting Focal Attention. J. Exp. Psychol. Hum. Percept. Perform. 18, 837-848. https://doi.org/10.1037/0096-1523.18.3.837

Chang, S., Egeth, H.E., 2021. Can salient stimuli really be suppressed? Attention, Perception, Psychophys. 83, 260-269. https://doi.org/10.3758/s13414-020-02207-8

Chelazzi, L., Eštočinová, J., Calletti, R., Gerfo, E. Lo, Sani, I., Libera, C. Della, Santandrea, E., 2014. Altering spatial priority maps via reward-based learning. J. Neurosci. 34, 8594-8604. https://doi.org/10.1523/JNEUROSCI.0277-14.2014

Chelazzi, L., Perlato, A., Santandrea, E., Della Libera, C., 2013. Rewards teach visual selective attention. Vision Res. 85, 58-72. https://doi.org/10.1016/J.VISRES.2012.12.005

Cheng, Saglam, A., André, S., Pooresmaeili, A., 2020. Cross-Modal Integration of Reward Value during Oculomotor Planning. eneuro 7, ENEURO.0381-19.2020. https://doi.org/10.1523/ENEURO.0381-19.2020

Corbetta, M., Miezin, F., Dobmeyer, S., Shulman, G., Petersen, S., 1990. Attentional modulation of neural processing of shape, color, and velocity in humans. Science (80-. ). 248, 1556-1559. https://doi.org/10.1126/SCIENCE.2360050

Corbetta, M., Shulman, G.L., 2002. Control of goal-directed and stimulus-driven attention in the brain. Nat. Rev. Neurosci. 3, 201-215. https://doi.org/10.1038/nrn755

Cornelio, P., Velasco, C., Obrist, M., 2021. Multisensory Integration as per Technological Advances: A Review. Front. Neurosci. https://doi.org/10.3389/fnins.2021.652611

Cox, S.M.L., Andrade, A., Johnsrude, I.S., 2005. Learning to like: A role for human orbitofrontal cortex in conditioned reward. J. Neurosci. 25, 2733-2740. https://doi.org/10.1523/JNEUROSCI.3360-04.2005

Dawkins, R., 2016. The selfish gene.

Della Libera, C., Chelazzi, L., 2009. Learning to Attend and to Ignore Is a Matter of Gains and Losses. Psychol. Sci. 20, 778-784. https://doi.org/10.1111/j.1467-9280.2009.02360.x

Della Libera, C., Chelazzi, L., 2006. Visual Selective Attention and the Effects of Monetary Rewards. Psychol. Sci. 17, 222-227. https://doi.org/10.1111/j.1467-9280.2006.01689.x

Delorme, A., Makeig, S., 2004. EEGLAB: An open source toolbox for analysis of single-trial EEG dynamics including independent component analysis. J. Neurosci. Methods. https://doi.org/10.1016/j.jneumeth.2003.10.009

Desimone, R., Duncan, J., 1995. NEURAL MECHANISMS OF SELECTIVE VISUAL ATTENTION, Annu. Rev. Neurosci.

Dickinson, A., Balleine, B., 1994. Motivational control of goal-directed action. Anim. Learn. Behav. 1994221 22, 1-18. https://doi.org/10.3758/BF03199951

Diederich, A., Colonius, H., 1987. Intersensory facilitation in the motor component? - A reaction time analysis. Psychol. Res. 49, 23-29. https://doi.org/10.1007/BF00309199

Driver, J., Noesselt, T., 2008. Multisensory Interplay Reveals Crossmodal Influences on "SensorySpecific" Brain Regions, Neural Responses, and Judgments. Neuron. https://doi.org/10.1016/j.neuron.2007.12.013

Duncan, J., 1984. Selective attention and the organization of visual information. J. Exp. Psychol. Gen. 113, 501-517. https://doi.org/10.1037/0096-3445.113.4.501

Duncan, J., Martens, S., Ward, R., 1997. Restricted attentional capacity within but not between sensory modalities. Nature 387, 808-810. https://doi.org/10.1038/42947

Dundon, N.M., Bertini, C., Làdavas, E., Sabel, B.A., Gall, C., 2015. Visual rehabilitation: Visual scanning, multisensory stimulation and vision restoration trainings. Front. Behav. Neurosci. https://doi.org/10.3389/fnbeh.2015.00192

Engelmann, J.B., Damaraju, E., Padmala, S., Pessoa, L., 2009. Combined effects of attention and motivation on visual task performance: Transient and sustained motivational effects. Front. Hum. Neurosci. 3, 4. https://doi.org/10.3389/neuro.09.004.2009

Eriksen, B.A., Eriksen, C.W., 1974. Effects of noise letters upon the identification of a target letter 
in a nonsearch task. Percept. Psychophys. 1974 161 16, 143-149. https://doi.org/10.3758/BF03203267

Etzel, J.A., Cole, M.W., Zacks, J.M., Kay, K.N., Braver, T.S., 2016. Reward Motivation Enhances Task Coding in Frontoparietal Cortex. Cereb. Cortex 26, 1647-1659. https://doi.org/10.1093/cercor/bhu327

Failing, M., Nissens, T., Pearson, D., Le Pelley, M., Theeuwes, J., 2015. Oculomotor capture by stimuli that signal the availability of reward. J. Neurophysiol. 114, 2316-2327. https://doi.org/10.1152/jn.00441.2015

Failing, M., Theeuwes, J., 2018. Selection history: How reward modulates selectivity of visual attention. Psychon. Bull. Rev. 25, 514-538. https://doi.org/10.3758/s13423-017-1380-y

Failing, M., Theeuwes, J., 2017. Don't let it distract you: how information about the availability of reward affects attentional selection. Attention, Perception, Psychophys. 79, 2275-2298. https://doi.org/10.3758/s13414-017-1376-8

Failing, M.F., Theeuwes, J., 2015. Nonspatial attentional capture by previously rewarded scene semantics. Vis. cogn. 23. https://doi.org/10.1080/13506285.2014.990546

Faul, F., Erdfelder, E., Lang, A.G., Buchner, A., 2007. G*Power 3: A flexible statistical power analysis program for the social, behavioral, and biomedical sciences, in: Behavior Research Methods. Psychonomic Society Inc., pp. 175-191. https://doi.org/10.3758/BF03193146

Feldmann-Wüstefeld, T., Schubö, A., 2013. Context homogeneity facilitates both distractor inhibition and target enhancement. J. Vis. 13. https://doi.org/10.1167/13.3.11

Feng, W., Stormer, V.S., Martinez, A., McDonald, J.J., Hillyard, S.A., 2014. Sounds Activate Visual Cortex and Improve Visual Discrimination. J. Neurosci. 34, 9817-9824. https://doi.org/10.1523/JNEUROSCI.4869-13.2014

Floresco, S.B., Magyar, O., 2006. Mesocortical dopamine modulation of executive functions: Beyond working memory. Psychopharmacology (Berl). https://doi.org/10.1007/s00213-0060404-5

Fox, C.R., Poldrack, R.A., 2009. Prospect theory and the brain, in: Neuroeconomics. Elsevier Inc., pp. 145-173. https://doi.org/10.1016/B978-0-12-374176-9.00011-7

Franzen, L., Delis, I., De Sousa, G., Kayser, C., Philiastides, M.G., 2020. Auditory information enhances post-sensory visual evidence during rapid multisensory decision-making. Nat. Commun. 11, 1-14. https://doi.org/10.1038/s41467-020-19306-7

Frassinetti, F., Bolognini, N., Làdavas, E., 2002. Enhancement of visual perception by crossmodal visuo-auditory interaction. Exp. Brain Res. 147, 332-343. https://doi.org/10.1007/s00221-002$1262-\mathrm{y}$

Frederick, S., Loewenstein, G., O’Donoghue, T., 2002. Time Discounting and Time Preference: A Critical Review. J. Econ. Lit. 40, 351-401. https://doi.org/10.1257/002205102320161311

Frens, M.A., Van Opstal, A.J., Van Der Willigen, R.F., 1995. Spatial and temporal factors determine auditory-visual interactions in human saccadic eye movements. Percept. Psychophys. 57, 802816. https://doi.org/10.3758/BF03206796

Friston, K., 2010. The free-energy principle: a unified brain theory? Nat. Rev. Neurosci. 2010112 11, 127-138. https://doi.org/10.1038/nrn2787

Garcia-Lazaro, H.G., Bartsch, M. V., Boehler, C.N., Krebs, R.M., Donohue, S.E., Harris, J.A., Schoenfeld, M.A., Hopf, J.M., 2018. Dissociating reward- and attention-driven biasing of global feature-based selection in human visual cortex. J. Cogn. Neurosci. 31, 469-481. https://doi.org/10.1162/jocn_a_01356

Gehring, W.J., Willoughby, A.R., 2002. The medial frontal cortex and the rapid processing of monetary gains and losses. Science (80-. ). 295, 2279-2282. https://doi.org/10.1126/science.1066893

Giard, M.H., Peronnet, F., 1999. Auditory-visual integration during multimodal object recognition in humans: A behavioral and electrophysiological study. J. Cogn. Neurosci. 11, 473-490. https://doi.org/10.1162/089892999563544

Glazer, J.E., Kelley, N.J., Pornpattananangkul, N., Mittal, V.A., Nusslock, R., 2018. Beyond the FRN: Broadening the time-course of EEG and ERP components implicated in reward processing. Int. J. Psychophysiol. https://doi.org/10.1016/j.ijpsycho.2018.02.002

Gold, J.I., Shadlen, M.N., 2007. The Neural Basis of Decision Making. Annu. Rev. Neurosci. 30, 535-574. https://doi.org/10.1146/annurev.neuro.29.051605.113038 
Goldring, J.E., Dorris, M.C., Corneil, B.D., Ballantyne, P.A., Munoz, D.P., 1996. Combined eyehead gaze shifts to visual and auditory targets in humans. Exp. Brain Res. 111, 68-78. https://doi.org/10.1007/BF00229557

Goldstein, R.Z., Cottone, L.A., Jia, Z., Maloney, T., Volkow, N.D., Squires, N.K., 2006. The effect of graded monetary reward on cognitive event-related potentials and behavior in young healthy adults. Int. J. Psychophysiol. 62, 272-279. https://doi.org/10.1016/j.ijpsycho.2006.05.006

Goltstein, P.M., Coffey, E.B.J., Roelfsema, P.R., Pennartz, C.M.A., 2013. In vivo two-photon Ca2+ imaging reveals selective reward effects on stimulus-specific assemblies in mouse visual cortex. J. Neurosci. 33, 11540-11555. https://doi.org/10.1523/JNEUROSCI.1341-12.2013

Goltstein, P.M., Meijer, G.T., Pennartz, C.M.A., 2018. Conditioning sharpens the spatial representation of rewarded stimuli in mouse primary visual cortex. Elife 7. https://doi.org/10.7554/eLife.37683

Gong, M., Yang, F., Li, S., 2016. Reward association facilitates distractor suppression in human visual search. Eur. J. Neurosci. 43, 942-953. https://doi.org/10.1111/ejn.13174

Grabenhorst, F., Rolls, E.T., Margot, C., 2011. A hedonically complex odor mixture produces an attentional capture effect in the brain. Neuroimage 55, 832-843. https://doi.org/10.1016/j.neuroimage.2010.12.023

Grégoire, L., Anderson, B.A., 2019. Semantic generalization of value-based attentional priority. Learn. Mem. 26, 460-464. https://doi.org/10.1101/lm.050336.119

Haber, S.N., 2011. Neuroanatomy of Reward: A View from the Ventral Striatum, Neurobiology of Sensation and Reward. CRC Press/Taylor \& Francis.

Halverson, H.M., 1927. The Upper Limit of Auditory Localization. Am. J. Psychol. 38, 97. https://doi.org/10.2307/1414648

Hammerschmidt, W., Kagan, I., Kulke, L., Schacht, A., 2018. Implicit reward associations impact face processing: Time-resolved evidence from event-related brain potentials and pupil dilations. Neuroimage 179, 557-569. https://doi.org/10.1016/j.neuroimage.2018.06.055

Henschke, J.U., Dylda, E., Katsanevaki, D., Dupuy, N., Currie, S.P., Amvrosiadis, T., Pakan, J.M.P., Rochefort, N.L., 2020. Reward Association Enhances Stimulus-Specific Representations in Primary Visual Cortex. Curr. Biol. 30, 1866-1880.e5. https://doi.org/10.1016/j.cub.2020.03.018

Herd, S., Krueger, K., Nair, A., Mollick, J., O’Reilly, R., 2021. Neural Mechanisms of Human Decision-Making. Cogn. Affect. Behav. Neurosci. 21, 35-57. https://doi.org/10.3758/s13415020-00842-0

Hickey, C., Chelazzi, L., Theeuwes, J., 2010a. Reward guides vision when it's your thing: Trait reward-seeking in reward-mediated visual priming. PLoS One 5, 14087. https://doi.org/10.1371/journal.pone.0014087

Hickey, C., Chelazzi, L., Theeuwes, J., 2010b. Reward changes salience in human vision via the anterior cingulate. J. Neurosci. 30, 11096-11103. https://doi.org/10.1523/JNEUROSCI.102610.2010

Hickey, C., Di Lollo, V., McDonald, J.J., 2009. Electrophysiological indices of target and distractor processing in visual search. J. Cogn. Neurosci. 21, 760-775. https://doi.org/10.1162/jocn.2009.21039

Hickey, C., Van Zoest, W., 2012. Reward creates oculomotor salience. Curr. Biol. https://doi.org/10.1016/j.cub.2012.02.007

Hoffman, J.E., Nelson, B., 1981. Spatial selectivity in visual search. Percept. Psychophys. 1981303 30, 283-290. https://doi.org/10.3758/BF03214284

Hollerman, J.R., Schultz, W., 1998. Dopamine neurons report an error in the temporal prediction of reward during learning. Nat. Neurosci. 1, 304-309. https://doi.org/10.1038/1124

Hopf, J.M., Boehler, C.N., Luck, S.J., Tsotsos, J.K., Heinze, H.J., Schoenfeld, M.A., 2006. Direct neurophysiological evidence for spatial suppression surrounding the focus of attention in vision. Proc. Natl. Acad. Sci. U. S. A. 103, 1053-1058. https://doi.org/10.1073/pnas.0507746103

Hughes, G., Mathan, S., Yeung, N., 2013. EEG indices of reward motivation and target detectability in a rapid visual detection task. Neuroimage 64, 590-600. https://doi.org/10.1016/j.neuroimage.2012.09.003

Hull, C., 1943. Principles of behavior: An introduction to behavior theory. Appleton-Century-Crofts, 
New York.

Itthipuripat, S., Vo, V.A., Sprague, T.C., Serences, J.T., 2019. Value-driven attentional capture enhances distractor representations in early visual cortex. PLoS Biol. 17, e3000186. https://doi.org/10.1371/journal.pbio.3000186

Itti, L., Koch, C., 2001. Computational modelling of visual attention. Nat. Rev. Neurosci. 2, 194203. https://doi.org/10.1038/35058500

Jay, T.M., 2003. Dopamine: A potential substrate for synaptic plasticity and memory mechanisms. Prog. Neurobiol. https://doi.org/10.1016/S0301-0082(03)00085-6

Kahneman, D., Treisman, A., Gibbs, B.J., 1992. The reviewing of object files: Object-specific integration of information. Cogn. Psychol. 24, 175-219. https://doi.org/10.1016/00100285(92)90007-O

Kahneman, D., Tversky, A., 2013. Choices, Values, and Frames. pp. 269-278. https://doi.org/10.1142/9789814417358_0016

Kang, G., Chang, W., Wang, L., Wei, P., Zhou, X., 2018. Reward enhances cross-modal conflict control in object categorization: Electrophysiological evidence. Psychophysiology 55, e13214. https://doi.org/10.1111/psyp.13214

Kang, G., Wang, L., Zhou, X., 2017. Reward interacts with modality shift to reduce cross-modal conflict. J. Vis. 17. https://doi.org/10.1167/17.1.19

Keitel, C., Maess, B., Schröger, E., Müller, M.M., 2013. Early visual and auditory processing rely on modality-specific attentional resources. Neuroimage 70, 240-249. https://doi.org/10.1016/j.neuroimage.2012.12.046

Kim, A.J., Lee, D.S., Anderson, B.A., 2021. Previously reward-associated sounds interfere with goaldirected auditory processing. Q. J. Exp. Psychol. 74, 1257-1263. https://doi.org/10.1177/1747021821990033

Kiss, M., Driver, J., Eimer, M., 2009. Reward Priority of Visual Target Singletons Modulates EventRelated Potential Signatures of Attentional Selection. Psychol. Sci. 20, 245-251. https://doi.org/10.1111/j.1467-9280.2009.02281.x

Klingel, M., Kopčo, N., Laback, B., 2021. Reweighting of Binaural Localization Cues Induced by Lateralization Training. JARO - J. Assoc. Res. Otolaryngol. 1-16. https://doi.org/10.1007/s10162-021-00800-8

Krebs, R.M., Boehler, C.N., Appelbaum, L.G., Woldorff, M.G., 2013. Reward Associations Reduce Behavioral Interference by Changing the Temporal Dynamics of Conflict Processing. PLoS One 8. https://doi.org/10.1371/journal.pone.0053894

Krebs, R.M., Boehler, C.N., Egner, T., Woldorff, M.G., 2011. The neural underpinnings of how reward associations can both guide and misguide attention. J. Neurosci. 31, 9752-9759. https://doi.org/10.1523/JNEUROSCI.0732-11.2011

Krebs, R.M., Boehler, C.N., Woldorff, M.G., 2010. The influence of reward associations on conflict processing in the Stroop task. Cognition 117, 341-347. https://doi.org/10.1016/j.cognition.2010.08.018

Kristjánsson, Á., Sigurjónsdóttir, Ó., Driver, J., 2010. Fortune and reversals of fortune in visual search: Reward contingencies for pop-out targets affect search efficiency and target repetition effects. Attention, Perception, Psychophys. 72, 1229-1236. https://doi.org/10.3758/APP.72.5.1229

Krugliakova, E., Gorin, A., Fedele, T., Shtyrov, Y., Moiseeva, V., Klucharev, V., Shestakova, A., 2019. The Monetary Incentive Delay (MID) Task Induces Changes in Sensory Processing: ERP Evidence. Front. Hum. Neurosci. 13. https://doi.org/10.3389/fnhum.2019.00382

Krugliakova, E., Klucharev, V., Fedele, T., Gorin, A., Kuznetsova, A., Shestakova, A., 2018. Correlation of cue-locked FRN and feedback-locked FRN in the auditory monetary incentive delay task. Exp. Brain Res. 236, 141-151. https://doi.org/10.1007/s00221-017-5113-2

Làdavas, E., Tosatto, L., Bertini, C., 2020. Behavioural and functional changes in neglect after multisensory stimulation. Neuropsychol. Rehabil. https://doi.org/10.1080/09602011.2020.1786411

Lakens, D., 2013. Calculating and reporting effect sizes to facilitate cumulative science: a practical primer for t-tests and ANOVAs. Front. Psychol. 4, 863. https://doi.org/10.3389/fpsyg.2013.00863

Le Pelley, M.E., Mitchell, C.J., Beesley, T., George, D.N., Wills, A.J., 2016. Attention and 
associative learning in humans: An integrative review. Psychol. Bull. 142, 1111-1140. https://doi.org/10.1037/bul0000064

Leo, F., Noppeney, U., 2014. Conditioned Sounds Enhance Visual Processing. PLoS One 9, e106860. https://doi.org/10.1371/journal.pone.0106860

Leo, F., Romei, V., Freeman, E., Ladavas, E., Driver, J., 2011. Looming sounds enhance orientation sensitivity for visual stimuli on the same side as such sounds. Exp. Brain Res. 213, 193-201. https://doi.org/10.1007/s00221-011-2742-8

Liu, D., Deng, J., Zhang, Z., Zhang, Z.Y., Sun, Y.G., Yang, T., Yao, H., 2020. Orbitofrontal control of visual cortex gain promotes visual associative learning. Nat. Commun. 11, 1-14. https://doi.org/10.1038/s41467-020-16609-7

Liu, J., Zhang, C., Zhu, Y., Liu, Y., Sun, H., Ristaniemi, T., Cong, F., Parviainen, T., 2020. Dissociable Effects of Reward on P300 and EEG Spectra Under Conditions of High vs. Low Vigilance During a Selective Visual Attention Task. Front. Hum. Neurosci. 14, 207. https://doi.org/10.3389/fnhum.2020.00207

Lockhofen, D.E.L., Mulert, C., 2021. Neurochemistry of Visual Attention. Front. Neurosci. 15, 517. https://doi.org/10.3389/fnins.2021.643597

Luck, S.J., Hillyard, S.A., Mouloua, M., Woldorff, M.G., et al, 1994. Effects of spatial cuing on luminance detectability: Psychophysical and electrophysiological evidence for early selection. J. Exp. Psychol. Hum. Percept. Perform. 20, 887-904. https://doi.org/10.1037//00961523.20.4.887

Luque, D., Beesley, T., Morris, R.W., Jack, B.N., Griffiths, O., Whitford, T.J., Le Pelley, M.E., 2017. Goal-directed and habit-like modulations of stimulus processing during reinforcement learning. J. Neurosci. 37, 3009-3017. https://doi.org/10.1523/JNEUROSCI.3205-16.2017

Luque, D., Morís, J., Rushby, J.A., Le Pelley, M.E., 2015. Goal-directed EEG activity evoked by discriminative stimuli in reinforcement learning. Psychophysiology 52, 238-248. https://doi.org/10.1111/psyp.12302

Maclean, M.H., Giesbrecht, B., 2015. Neural evidence reveals the rapid effects of reward history on selective attention. Brain Res. 1606, 86-94. https://doi.org/10.1016/j.brainres.2015.02.016

Macmillan, N.A., Creelman, C.D., 1991. Detection theory: A user's guide., Detection theory: A user's guide. Cambridge University Press, New York, NY, US.

Maier, M., Ballester, B.R., Verschure, P.F.M.J., 2019. Principles of Neurorehabilitation After Stroke Based on Motor Learning and Brain Plasticity Mechanisms. Front. Syst. Neurosci. https://doi.org/10.3389/fnsys.2019.00074

Mangun, G.R., 1995. Neural mechanisms of visual selective attention. Psychophysiology 32, 4-18. https://doi.org/10.1111/j.1469-8986.1995.tb03400.x

Manohar, S.G., Chong, T.T.J., Apps, M.A.J., Batla, A., Stamelou, M., Jarman, P.R., Bhatia, K.P., Husain, M., 2015. Reward Pays the Cost of Noise Reduction in Motor and Cognitive Control. Curr. Biol. 25, 1707-1716. https://doi.org/10.1016/j.cub.2015.05.038

Manohar, S.G., Muhammed, K., Fallon, S.J., Husain, M., 2019. Motivation dynamically increases noise resistance by internal feedback during movement. Neuropsychologia 123, 19-29. https://doi.org/10.1016/j.neuropsychologia.2018.07.011

Maunsell, J.H.R., 2004. Neuronal representations of cognitive state: Reward or attention? Trends Cogn. Sci. 8, 261-265. https://doi.org/10.1016/j.tics.2004.04.003

McDonald, J.J., Störmer, V.S., Martinez, A., Feng, W., Hillyard, S.A., 2013. Salient Sounds Activate Human Visual Cortex Automatically. J. Neurosci. 33, 9194.

Meredith, M.A., Stein, B.E., 1986. Visual, auditory, and somatosensory convergence on cells in superior colliculus results in multisensory integration. J. Neurophysiol. 56, 640-662. https://doi.org/10.1152/jn.1986.56.3.640

Mine, C., Saiki, J., 2015. Task-irrelevant stimulus-reward association induces value-driven attentional capture. Attention, Perception, Psychophys. 77, 1896-1907. https://doi.org/10.3758/s13414-015-0894-5

Mogami, T., Tanaka, K., 2006. Reward association affects neuronal responses to visual stimuli in macaque TE and perirhinal cortices. J. Neurosci. 26, 6761-6770. https://doi.org/10.1523/JNEUROSCI.4924-05.2006

Mognon, A., Jovicich, J., Bruzzone, L., Buiatti, M., 1994. ADJUST: An automatic EEG artifact detector based on the joint use of spatial and temporal features. Psychophysiology. 
https://doi.org/10.1111/j.1469-8986.2010.01061.x

Molholm, S., Ritter, W., Murray, M.M., Javitt, D.C., Schroeder, C.E., Foxe, J.J., 2002. Multisensory auditory-visual interactions during early sensory processing in humans: A high-density electrical mapping study, in: Cognitive Brain Research. Elsevier, pp. 115-128. https://doi.org/10.1016/S0926-6410(02)00066-6

Motter, B.C., 1993. Focal attention produces spatially selective processing in visual cortical areas V1, V2, and V4 in the presence of competing stimuli. J. Neurophysiol. 70, 909-919. https://doi.org/10.1152/jn.1993.70.3.909

Mounts, J.R.W., 2000. Attentional capture by abrupt onsets and feature singletons produces inhibitory surrounds. Percept. Psychophys. 62, 1485-1493. https://doi.org/10.3758/BF03212148

Nakayama, K., 1990. The iconic bottleneck and the tenuous link between early visual processing and perception, in: Blakemore, C. (Ed.), Vision: Coding and Efficiency. Cambridge: Cambridge University Press, pp. 411-422.

Navarra, J., Alsius, A., Soto-Faraco, S., Spence, C., 2010. Assessing the role of attention in the audiovisual integration of speech. Inf. Fusion 11, 4-11. https://doi.org/10.1016/j.inffus.2009.04.001

Ngo, M.K., Spence, C., 2010. Crossmodal facilitation of masked visual target identification. Attention, Perception, Psychophys. 72, 1938-1947. https://doi.org/10.3758/APP.72.7.1938

Niv, Y., Joel, D., Dayan, P., 2006. A normative perspective on motivation. Trends Cogn. Sci. 10, 375-381. https://doi.org/10.1016/J.TICS.2006.06.010

Nobre, A.C., Rao, A., Chelazzi, L., 2006. Selective attention to specific features within objects: Behavioral and electrophysiological evidence. J. Cogn. Neurosci. 18, 539-561. https://doi.org/10.1162/jocn.2006.18.4.539

Novak, K.D., Foti, D., 2015. Teasing apart the anticipatory and consummatory processing of monetary incentives: An event-related potential study of reward dynamics. Psychophysiology 52, 1470-1482. https://doi.org/10.1111/psyp.12504

O'Doherty, J.P., Cockburn, J., Pauli, W.M., 2017. Learning, Reward, and Decision Making. Annu. Rev. Psychol. 68, 73-100. https://doi.org/10.1146/annurev-psych-010416-044216

Otto, A.R., Daw, N.D., 2019. The opportunity cost of time modulates cognitive effort. Neuropsychologia 123, 92-105. https://doi.org/10.1016/j.neuropsychologia.2018.05.006

Padmala, S., Pessoa, L., 2010. Interactions between cognition and motivation during response inhibition. Neuropsychologia

48 ,

$558-565$. https://doi.org/10.1016/j.neuropsychologia.2009.10.017

Pavlov, 1927. Conditioned Reflexes. Oxford University Press, Oxford.

Pearson, D., Watson, P., Cheng, P.X., Le Pelley, M.E., 2020. Overt Attentional Capture by RewardRelated Stimuli Overcomes Inhibitory Suppression. J. Exp. Psychol. Hum. Percept. Perform. https://doi.org/10.1037/xhp0000728

Pessiglione, M., Schmidt, L., Draganski, B., Kalisch, R., Lau, H., Dolan, R.J., Frith, C.D., 2007. How the Brain Translates Money into Force: A Neuroimaging Study of Subliminal Motivation. Science 316, 904. https://doi.org/10.1126/SCIENCE.1140459

Pessiglione, M., Seymour, B., Flandin, G., Dolan, R.J., Frith, C.D., 2006. Dopamine-dependent prediction errors underpin reward-seeking behaviour in humans. Nature 442, 1042-1045. https://doi.org/10.1038/nature05051

Pessiglione, M., Vinckier, F., Bouret, S., Daunizeau, J., Le Bouc, R., 2018. Why not try harder? Computational approach to motivation deficits in neuro-psychiatric diseases, Brain.

Pessoa, L., 2015. Multiple influences of reward on perception and attention. Vis. cogn. 23, 272-290. https://doi.org/10.1080/13506285.2014.974729

Pessoa, L., 2009. How do emotion and motivation direct executive control? Trends Cogn. Sci. 13, 160-166. https://doi.org/10.1016/j.tics.2009.01.006

Pessoa, L., Engelmann, J.B., 2010. Embedding reward signals into perception and cognition. Front. Neurosci. 4, 17. https://doi.org/10.3389/fnins.2010.00017

Peters, J., Büchel, C., 2010. Neural representations of subjective reward value. Behav. Brain Res. 213, 135-141. https://doi.org/10.1016/J.BBR.2010.04.031

Pfabigan, D.M., Seidel, E.M., Sladky, R., Hahn, A., Paul, K., Grahl, A., Küblböck, M., Kraus, C., Hummer, A., Kranz, G.S., Windischberger, C., Lanzenberger, R., Lamm, C., 2014. P300 
amplitude variation is related to ventral striatum BOLD response during gain and loss anticipation: An EEG and fMRI experiment. Neuroimage 96, 12-21. https://doi.org/10.1016/j.neuroimage.2014.03.077

Pick, H.L., Warren, D.H., Hay, J.C., 1969. Sensory conflict in judgments of spatial direction. Percept. Psychophys. 6, 203-205. https://doi.org/10.3758/BF03207017

Platt, M.L., Glimcher, P.W., 1999. Neural correlates of decision variables in parietal cortex. Nature 400, 233-238. https://doi.org/10.1038/22268

Pleger, B., Blankenburg, F., Ruff, C.C., Driver, J., Dolan, R.J., 2008. Reward facilitates tactile judgments and modulates hemodynamic responses in human primary somatosensory cortex. J. Neurosci. 28, 8161-8168. https://doi.org/10.1523/JNEUROSCI.1093-08.2008

Pool, E., Brosch, T., Delplanque, S., Sander, D., 2014. Where is the chocolate? Rapid spatial orienting toward stimuli associated with primary rewards. Cognition 130, 348-359. https://doi.org/10.1016/j.cognition.2013.12.002

Pooresmaeili, A., FitzGerald, T.H.B., Bach, D.R., Toelch, U., Ostendorf, F., Dolan, R.J., 2014. Cross-modal effects of value on perceptual acuity and stimulus encoding. Proc. Natl. Acad. Sci. U. S. A. 111, 15244-15249. https://doi.org/10.1073/pnas.1408873111

Pooresmaeili, A., Roelfsema, P.R., 2014. A growth-cone model for the spread of object-based attention during contour grouping. Curr. Biol. 24. https://doi.org/10.1016/j.cub.2014.10.007

Pornpattananangkul, N., Nusslock, R., 2015. Motivated to win: Relationship between anticipatory and outcome reward-related neural activity. Brain Cogn. 100, 21-40. https://doi.org/10.1016/j.bandc.2015.09.002

Posner, M.I., 1980. Orienting of Attention. Q. J. Exp. Psychol. 32, 3-25. https://doi.org/10.1080/00335558008248231

Qin, N., Gu, R., Xue, J., Chen, C., Zhang, M., 2021. Reward-driven attention alters perceived salience. J. Vis. 21, 1-13. https://doi.org/10.1167/JOV.21.1.7

Rangel, A., Camerer, C., Montague, P.R., 2008. A framework for studying the neurobiology of valuebased decision making. Nat. Rev. Neurosci. https://doi.org/10.1038/nrn2357

Raymond, J.E., O'Brien, J.L., 2009. Selective visual attention and motivation: the consequences of value learning in an attentional blink task. Psychol. Sci. 20, 981-8. https://doi.org/10.1111/j.1467-9280.2009.02391.x

Reynolds, J.H., Chelazzi, L., 2004. Attentional modulation of visual processing. Annu. Rev. Neurosci. https://doi.org/10.1146/annurev.neuro.26.041002.131039

Reynolds, J.H., Chelazzi, L., Desimone, R., 1999. Competitive mechanisms subserve attention in macaque areas V2 and V4. J. Neurosci. 19, 1736-1753. https://doi.org/10.1523/jneurosci.1905-01736.1999

Rock, I., Gutman, D., 1981. The effect of inattention on form perception. J. Exp. Psychol. Hum. Percept. Perform. 7, 275-285. https://doi.org/10.1037/0096-1523.7.2.275

Rolls, E.T., 2015. Taste, olfactory, and food reward value processing in the brain. Prog. Neurobiol. https://doi.org/10.1016/j.pneurobio.2015.03.002

Rossi, V., Vanlessen, N., Bayer, M., Grass, A., Pourtois, G., Schacht, A., 2017. Motivational salience modulates early visual cortex responses across task sets. J. Cogn. Neurosci. 29, 968-979. https://doi.org/10.1162/jocn_a_01093

Rusz, D., Bijleveld, E., Kompier, M.A.J., 2018. Reward-associated distractors can harm cognitive performance. PLoS One 13. https://doi.org/10.1371/journal.pone.0205091

Rusz, D., Le Pelley, M.E., Kompier, M.A.J., Mait, L., Bijleveld, E., 2020. Reward-Driven distraction: A meta-analysis. Psychol. Bull. 146, 872-899.

Rutherford, H.J.V., O'Brien, J.L., Raymond, J.E., 2010. Value associations of irrelevant stimuli modify rapid visual orienting. Psychon. Bull. Rev. 17, 536-542. https://doi.org/10.3758/PBR.17.4.536

Rutkowski, R.G., Weinberger, N.M., 2005. Encoding of learned importance of sound by magnitude of representational area in primary auditory cortex. Proc. Natl. Acad. Sci. U. S. A. 102, 1366413669. https://doi.org/10.1073/pnas.0506838102

San Martín, R., Appelbaum, L.G., Huettel, S.A., Woldorff, M.G., 2016. Cortical Brain Activity Reflecting Attentional Biasing Toward Reward-Predicting Cues Covaries with Economic Decision-Making Performance. Cereb. Cortex 26, 1-11. https://doi.org/10.1093/cercor/bhu160

Sanz, L.R.D., Vuilleumier, P., Bourgeois, A., 2018. Cross-modal integration during value-driven 
attentional capture. Neuropsychologia

https://doi.org/10.1016/j.neuropsychologia.2018.10.014

Sawaki, R., Luck, S.J., Raymond, J.E., 2015. How attention changes in response to incentives. J. Cogn. Neurosci. 27, 2229-2239. https://doi.org/10.1162/jocn_a_00847

Schacht, A., Adler, N., Chen, P., Guo, T., Sommer, W., 2012. Association with positive outcome induces early effects in event-related brain potentials. Biol. Psychol. 89, 130-136. https://doi.org/10.1016/j.biopsycho.2011.10.001

Schultz, W., 2015. Neuronal reward and decision signals: From theories to data. Physiol. Rev. 95, 853-951. https://doi.org/10.1152/physrev.00023.2014

Schultz, W., 2013. Updating dopamine reward signals. Curr. Opin. Neurobiol. 23, 229-238. https://doi.org/10.1016/J.CONB.2012.11.012

Schultz, W., 2006. Behavioral Theories and the Neurophysiology of Reward. Annu. Rev. Psychol. 57, 87-115. https://doi.org/10.1146/annurev.psych.56.091103.070229

Schultz, W., 2002. Getting Formal with Dopamine and Reward. Neuron 36, 241-263. https://doi.org/10.1016/S0896-6273(02)00967-4

Schultz, W., 2000. Multiple reward signals in the brain 1, 199-207. https://doi.org/10.1038/35044563

Schultz, W., 1998. Predictive Reward Signal of Dopamine Neurons. J. Neurophysiol. 80, 1-27. https://doi.org/10.1152/jn.1998.80.1.1

Schultz, W., 1986. Responses of midbrain dopamine neurons to behavioral trigger stimuli in the monkey. J. Neurophysiol. 56, 1439-1461. https://doi.org/10.1152/jn.1986.56.5.1439

Schultz, W., Dayan, P., Montague, P.R., 1997. A Neural Substrate of Prediction and Reward. Science (80-. ). 275, 1593-1599. https://doi.org/10.1126/science.275.5306.1593

Seitz, A.R., Kim, R., Shams, L., 2006. Sound Facilitates Visual Learning. Curr. Biol. 16, 1422-1427. https://doi.org/10.1016/j.cub.2006.05.048

Serences, J.T., 2008. Value-Based Modulations in Human Visual Cortex. Neuron 60, 1169-1181. https://doi.org/10.1016/j.neuron.2008.10.051

Serences, J.T., Saproo, S., 2010. Population response profiles in early visual cortex are biased in favor of more valuable stimuli. J. Neurophysiol. 104, 76-87. https://doi.org/10.1152/jn.01090.2009

Serences, J.T., Saproo, S., Scolari, M., Ho, T., Muftuler, L.T., 2009. Estimating the influence of attention on population codes in human visual cortex using voxel-based tuning functions. Neuroimage 44, 223-231. https://doi.org/10.1016/J.NEUROIMAGE.2008.07.043

Shadmehr, R., Xivry, J.J.O. de, Xu-Wilson, M., Shih, T.-Y., 2010. Temporal Discounting of Reward and the Cost of Time in Motor Control. J. Neurosci. 30, 10507-10516. https://doi.org/10.1523/JNEUROSCI.1343-10.2010

Shams, L., Kamitani, Y., Shimojo, S., 2000. Illusions: What you see is what you hear. Nature 408, 788. https://doi.org/10.1038/35048669

Shams, L., Seitz, A.R., 2008. Benefits of multisensory learning. Trends Cogn. Sci. 12, 411-417. https://doi.org/10.1016/j.tics.2008.07.006

Shapcott, K.A., Schmiedt, J.T., Kouroupaki, K., Kienitz, R., Lazar, A., Singer, W., Schmid, M.C., 2020. Reward-related suppression of neural activity in macaque visual area v4. Cereb. Cortex 30, 4871-4881. https://doi.org/10.1093/cercor/bhaa079

Shuler, M.G., Bear, M.F., 2006. Reward timing in the primary visual cortex. Science (80-. ). 311, 1606-1609. https://doi.org/10.1126/science.1123513

Slutsky, D.A., Recanzone, G.H., 2001. Temporal and spatial dependency of the ventriloquism effect. Neuroreport 12, 7-10. https://doi.org/10.1097/00001756-200101220-00009

Sobal, J., 1998. Cultural Comparison Research Designs in Food, Eating, and Nutrition. Food Qual. Prefer. 9, 385-392. https://doi.org/10.1016/S0950-3293(98)00029-9

Stanisor, L., Van Der Togt, C., Pennartz, C.M.A., Roelfsema, P.R., 2013. A unified selection signal for attention and reward in primary visual cortex. Proc. Natl. Acad. Sci. U. S. A. 110, 91369141. https://doi.org/10.1073/pnas.1300117110

Stein, B.E., Meredith, M.A., 1993. The merging of the senses. The MIT Press.

Steinberg, E.E., Keiflin, R., Boivin, J.R., Witten, I.B., Deisseroth, K., Janak, P.H., 2013. A causal link between prediction errors, dopamine neurons and learning. Nat. Neurosci. 16, 966-973. https://doi.org/10.1038/nn.3413 
Stine, G.M., Zylberberg, A., Ditterich, J., Shadlen, M.N., 2020. Differentiating between integration and non-integration strategies in perceptual decision making. Elife 9. https://doi.org/10.7554/eLife.55365

Störmer, V.S., Alvarez, G.A., 2014. Feature-based attention elicits surround suppression in feature space. Curr. Biol. 24, 1985-1988. https://doi.org/10.1016/j.cub.2014.07.030

Störmer, V.S., McDonald, J.J., Hillyard, S.A., 2009. Cross-modal cueing of attention alters appearance and early cortical processing of visual stimuli. Proc. Natl. Acad. Sci. U. S. A. 106, 22456-22461. https://doi.org/10.1073/pnas.0907573106

Sutton, R.S., Barto, A.G., 2018. Reinforcement Learning: An Introduction. MIT Press, Cambridge; MA.

Takakuwa, N., Kato, R., Redgrave, P., Isa, T., 2017. Emergence of visually-evoked reward expectation signals in dopamine neurons via the superior colliculus in V1 lesioned monkeys. Elife 6. https://doi.org/10.7554/eLife.24459

Takikawa, Y., Kawagoe, R., Itoh, H., Nakahara, H., Hikosaka, O., 2002. Modulation of saccadic eye movements by predicted reward outcome. Exp. Brain Res. 142. https://doi.org/10.1007/s00221001-0928-1

Talsma, D., Doty, T.J., Woldorff, M.G., 2007. Selective attention and audiovisual integration: Is attending to both modalities a prerequisite for early integration? Cereb. Cortex 17, 679-690. https://doi.org/10.1093/cercor/bhk016

Talsma, D., Woldorff, M.G., 2005. Selective attention and multisensory integration: Multiple phases of effects on the evoked brain activity. J. Cogn. Neurosci. 17, 1098-1114. https://doi.org/10.1162/0898929054475172

Tankelevitch, L., Spaak, E., Rushworth, M., Stokes, M., 2019. Previously reward-associated stimuli capture spatial attention in the absence of changes in the corresponding sensory representations as measured with MEG. bioRxiv 622589. https://doi.org/10.1101/622589

Tankelevitch, L., Spaak, E., Rushworth, M.F.S., Stokes, M.G., 2020. Previously Reward-Associated Stimuli Capture Spatial Attention in the Absence of Changes in the Corresponding Sensory Representations as Measured with MEG. J. Neurosci. 40, 5033-5050. https://doi.org/10.1523/JNEUROSCI.1172-19.2020

Teder-Sälejärvi, W.A., Di Russo, F., McDonald, J.J., Hillyard, S.A., 2005. Effects of spatial congruity on audio-visual multimodal integration. J. Cogn. Neurosci. 17, 1396-1409. https://doi.org/10.1162/0898929054985383

Teder-Sälejärvi, W.A., McDonald, J.J., Di Russo, F., Hillyard, S.A., 2002. An analysis of audiovisual crossmodal integration by means of event-related potential (ERP) recordings, in: Cognitive Brain Research. https://doi.org/10.1016/S0926-6410(02)00065-4

Theeuwes, J., 2019. Goal-driven, stimulus-driven, and history-driven selection. Curr. Opin. Psychol. https://doi.org/10.1016/j.copsyc.2018.12.024

Theeuwes, J., 2018. Visual selection: Usually fast and automatic; Seldom slow and volitional. J. Cogn. 1. https://doi.org/10.5334/joc.13

Theeuwes, J., Belopolsky, A. V., 2012. Reward grabs the eye: Oculomotor capture by rewarding stimuli. Vision Res. 74, 80-85. https://doi.org/10.1016/j.visres.2012.07.024

Thorndike, E.L., 1911. Animal intelligence. Macmillan, New York.

Trappenberg, T.P., Dorris, M.C., Munoz, D.P., Klein, R.M., 2001. A model of saccade initiation based on the competitive integration of exogenous and endogenous signals in the superior colliculus. J. Cogn. Neurosci. 13, 256-271. https://doi.org/10.1162/089892901564306

Vakhrushev, R., Cheng, F., Schacht, A., Pooresmaeili, A., 2021. Differential effects of intra-modal and cross-modal reward value on visual perception: ERP evidence. bioRxiv 2021.09.29.462374. https://doi.org/10.1101/2021.09.29.462374

Van Den Berg, B., Krebs, R.M., Lorist, M.M., Woldorff, M.G., 2014. Utilization of reward-prospect enhances preparatory attention and reduces stimulus conflict. Cogn. Affect. Behav. Neurosci. 14, 561-577. https://doi.org/10.3758/s13415-014-0281-Z

Van Zoest, W., Donk, M., Theeuwes, J., 2004. The role of stimulus-driven and goal-driven control in saccadic visual selection. J. Exp. Psychol. Hum. Percept. Perform. 30, 746-759. https://doi.org/10.1037/0096-1523.30.4.749

Vitay, J., Hamker, F.H., 2007. On the role of dopamine in cognitive vision, in: Lecture Notes in Computer Science (Including Subseries Lecture Notes in Artificial Intelligence and Lecture 
Notes in Bioinformatics). Springer Verlag, pp. 352-366. https://doi.org/10.1007/978-3-54077343-6_23

Von Kriegstein, K., Giraud, A.L., 2006. Implicit multisensory associations influence voice recognition. PLoS Biol. 4, 1809-1820. https://doi.org/10.1371/journal.pbio.0040326

Wang, L., Yu, H., Zhou, X., 2013. Interaction between value and perceptual salience in value-driven attentional capture. J. Vis. 13, 5-5. https://doi.org/10.1167/13.3.5

Watson, A.B., Pelli, D.G., 1983. Quest: A Bayesian adaptive psychometric method. Percept. Psychophys. 33, 113-120. https://doi.org/10.3758/BF03202828

Watson, P., Pearson, D., Most, S.B., Theeuwes, J., Wiers, R.W., Le Pelley, M.E., 2019. Attentional capture by Pavlovian reward-signalling distractors in visual search persists when rewards are removed. PLoS One 14, e0226284. https://doi.org/10.1371/journal.pone.0226284

Watson, R., Latinus, M., Noguchi, T., Garrod, O., Crabbe, F., Belin, P., 2014. Crossmodal adaptation in right posterior superior temporal sulcus during face-voice emotional integration. J. Neurosci. 34, 6813-6821. https://doi.org/10.1523/JNEUROSCI.4478-13.2014

Weil, R.S., Furl, N., Ruff, C.C., Symmonds, M., Flandin, G., Dolan, R.J., Driver, J., Rees, G., 2010. Rewarding Feedback After Correct Visual Discriminations Has Both General and Specific Influences on Visual Cortex. J. Neurophysiol. 104, 1746-1757. https://doi.org/10.1152/jn.00870.2009

Weinberger, N.M., 2007. Associative representational plasticity in the auditory cortex: A synthesis of two disciplines. Learn. Mem. https://doi.org/10.1101/lm.421807

Welch, R.B., Warren, D.H., 1980. Immediate perceptual response to intersensory discrepancy. Psychol. Bull. 88, 638-667. https://doi.org/10.1037/0033-2909.88.3.638

Westbrook, A., Frank, M.J., Cools, R., 2021. A mosaic of cost-benefit control over cortico-striatal circuitry. Trends Cogn. Sci. https://doi.org/10.1016/j.tics.2021.04.007

Wikman, P., Rinne, T., Petkov, C.I., 2019. Reward cues readily direct monkeys' auditory performance resulting in broad auditory cortex modulation and interaction with sites along cholinergic and dopaminergic pathways. Sci. Rep. 9. https://doi.org/10.1038/s41598-01938833-y

Wild, B., Treue, S., 2021. Primate extrastriate cortical area MST: a gateway between sensation and cognition. J. Neurophysiol. 125, 1851-1882. https://doi.org/10.1152/jn.00384.2020

Wise, R.A., 2004. Dopamine, learning and motivation. Nat. Rev. Neurosci. https://doi.org/10.1038/nrn1406

Wittmann, M., Leland, D.S., Paulus, M.P., 2007. Time and decision making: differential contribution of the posterior insular cortex and the striatum during a delay discounting task. Exp. Brain Res. 20061794 179, 643-653. https://doi.org/10.1007/S00221-006-0822-Y

Woldorff, M.G., 1993. Distortion of ERP averages due to overlap from temporally adjacent ERPs: Analysis and correction. Psychophysiology 30, 98-119. https://doi.org/10.1111/j.14698986.1993.tb03209.x

Woldorff, M.G., Fox, P.T., Matzke, M., Lancaster, J.L., Veeraswamy, S., Zamarripa, F., Seabolt, M., Glass, T., Gao, J.H., Martin, C.C., Jerabek, P., 1997. Retinotopic organization of early visual spatial attention effects as revealed by PET and ERPs, in: Human Brain Mapping. Hum Brain Mapp, pp. 280-286. https://doi.org/10.1002/(SICI)1097-0193(1997)5:4<280::AIDHBM13>3.0.CO;2-I

Wolfe, J.M., 2021. Guided Search 6.0: An updated model of visual search. Psychon. Bull. Rev. 28, 1060-1092. https://doi.org/10.3758/s13423-020-01859-9

Woods, T.M., Recanzone, G.H., 2004. Cross-Modal Interactions Evidenced, in: The Handbook of Multisensory Processes. MIT Press, Cambridge, MA, pp. 35-48.

Yantis, S., Anderson, B.A., Wampler, E.K., Laurent, P.A., 2012. Reward and attentional control in visual search. Nebraska Symp. Motiv. https://doi.org/10.1007/978-1-4614-4794-8-5

Yeung, N., Sanfey, A.G., 2004. Independent coding of reward magnitude and valence in the human brain. J. Neurosci. 24, 6258-6264. https://doi.org/10.1523/JNEUROSCI.4537-03.2004

Zelinsky, G.J., Bisley, J.W., 2015. The what, where, and why of priority maps and their interactions with visual working memory. Ann. N. Y. Acad. Sci. 1339, 154-164. https://doi.org/10.1111/nyas.12606

Zimmer, U., Itthipanyanan, S., Grent-’T-Jong, T., Woldorff, M.G., 2010. The electrophysiological time course of the interaction of stimulus conflict and the multisensory spread of attention. Eur. 
J. Neurosci. 31, 1744-1754. https://doi.org/10.1111/j.1460-9568.2010.07229.x 